Supporting Information

\title{
Synthesis of 2-Azapyrenes and their Photophysical and Electrochemical Properties
}

Ricardo Molenda ${ }^{\mathrm{a}}$, Sebastian Boldt ${ }^{\mathrm{a}}$, Alexander Villinger ${ }^{\mathrm{a}}$, Peter Ehlers ${ }^{\mathrm{a}, \mathrm{b}}$, Peter Langer ${ }^{\mathrm{a}, \mathrm{b} *}$

a Universität Rostock, Institut für Chemie, A.-Einstein-Str. 3a, 18059 Rostock, Germany

Tel.: +49381498 6410, Fax : +493814986412.

${ }^{b}$ Leibniz Institut für Katalyse an der Universität Rostock, A.-Einstein-Str. 29a, 18059 Rostock, Germany

*Corresponding author: peter.langer@uni-rostock.de

\section{List of Contents}

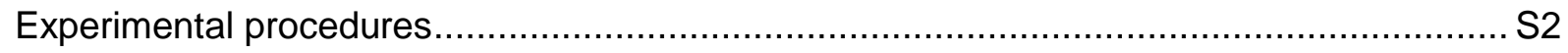

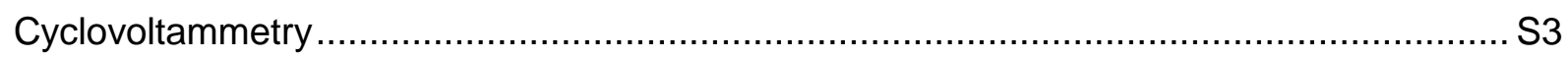

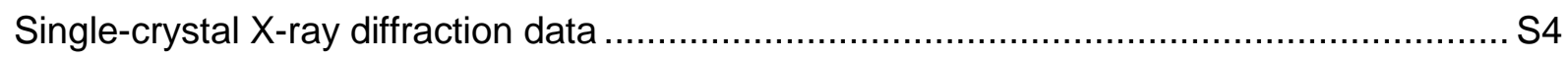

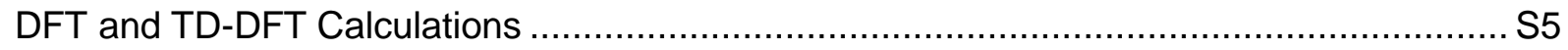

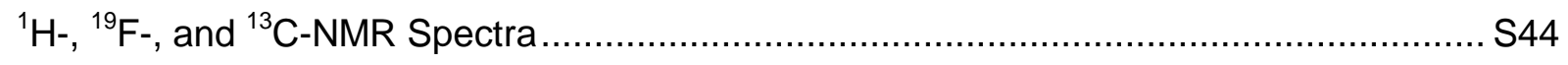




\section{Experimental procedures}

Table S1: Optimization of the Sonogashira-cross coupling reaction for $\mathbf{2 a}$.

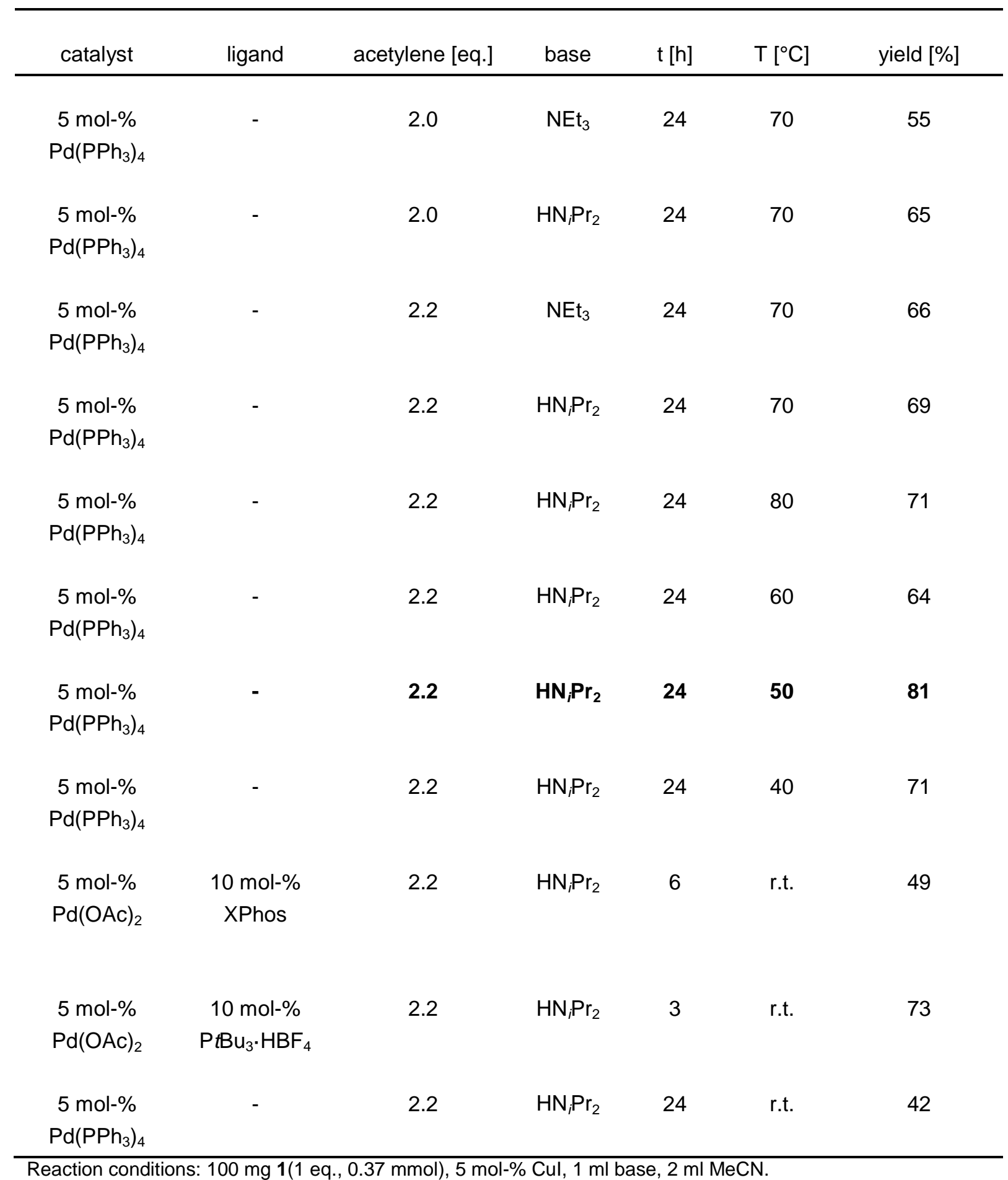


Table S2: Optimization of the Suzuki-cross coupling reaction for $3 \mathbf{b}$.

\begin{tabular}{|c|c|c|c|c|c|c|}
\hline catalyst & ligand & $\begin{array}{c}\text { boronic acid } \\
\text { [eq.] }\end{array}$ & solvent & $\mathrm{t}[\mathrm{h}]$ & $\mathrm{T}\left[{ }^{\circ} \mathrm{C}\right]$ & yield [\%] \\
\hline $\begin{array}{r}5 \mathrm{~mol}^{-} \% \\
\mathrm{Pd}\left(\mathrm{PPh}_{3}\right)_{4}\end{array}$ & - & 1.5 & $\mathrm{DMF} / \mathrm{H}_{2} \mathrm{O}$ & 24 & 120 & 70 \\
\hline $\begin{array}{c}5 \mathrm{~mol}-\% \\
\mathrm{Pd}(\mathrm{OAc})_{2}\end{array}$ & $\begin{array}{c}10 \text { mol-\% } \\
\text { SPhos }\end{array}$ & 1.5 & $\mathrm{DMF} / \mathrm{H}_{2} \mathrm{O}$ & 24 & 120 & 30 \\
\hline $\begin{array}{c}5 \mathrm{~mol}^{-} \% \\
\mathrm{Pd}\left(\mathrm{PPh}_{3}\right)_{4}\end{array}$ & - & 2.0 & $\mathrm{DMF} / \mathrm{H}_{2} \mathrm{O}$ & 24 & 120 & 76 \\
\hline $\begin{array}{c}5 \mathrm{~mol}^{-} \% \\
\mathrm{Pd}\left(\mathrm{PPh}_{3}\right)_{4}\end{array}$ & - & 2.0 & 1,4-Dioxane & 24 & 90 & 85 \\
\hline
\end{tabular}

Reaction conditions: $100 \mathrm{mg} \mathrm{2a}(1$ eq., $0.319 \mathrm{mmol}), 2$ eq. $\mathrm{K}_{3} \mathrm{PO}_{4}$.

\section{Cyclovoltammetry}

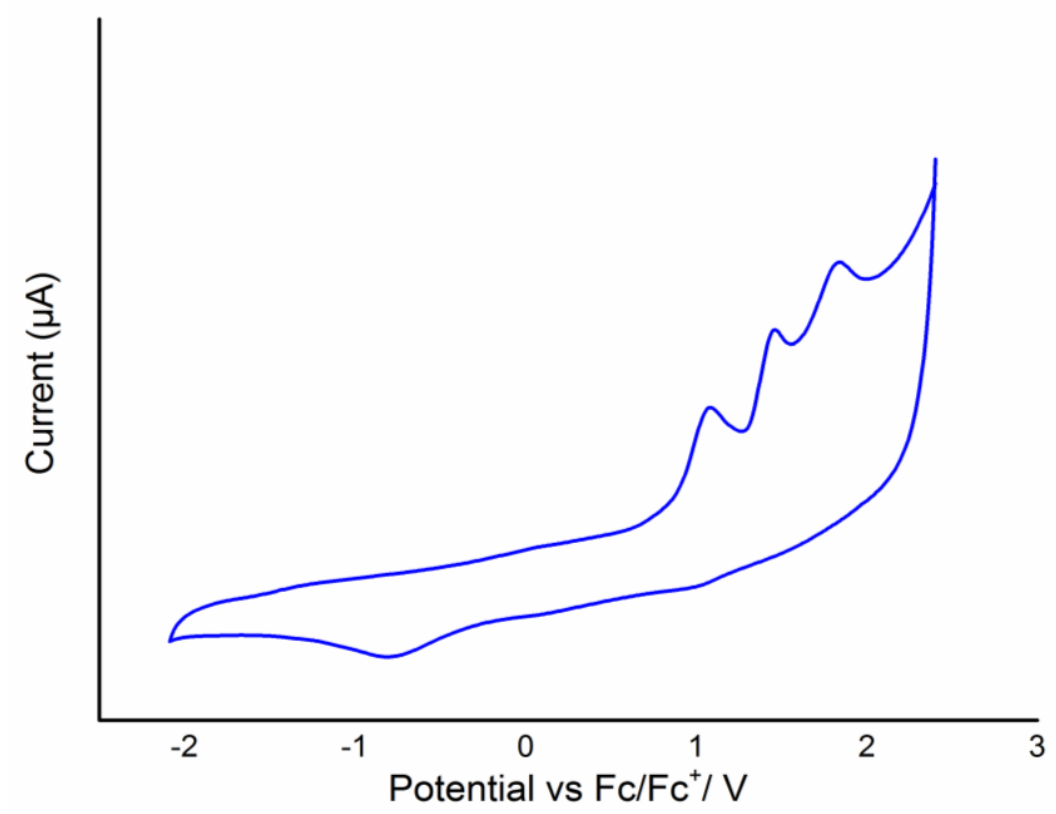

Figure S1. Cyclic voltammogram of 5 a measured in Acetonitrile with $0.1 \mathrm{M} \mathrm{n}$-Bu $\mathrm{NPF}_{6}$ as a supporting electrolyte, glassy carbon working electrode, ANE2 as reference electrode and Pt counter-electrode with ferrocene as standard at a scan rate of $100 \mathrm{mV} \mathrm{s}^{-1}$. 


\section{Single-crystal X-ray diffraction data}

Crystals of $\mathbf{4 j}$ and $\mathbf{5 b}$ were obtained by slow evaporation of chloroform at $20^{\circ} \mathrm{C}$. X-ray single crystal structure analysis was performed on a Bruker Apex Kappa-II CCD-diffractometer.

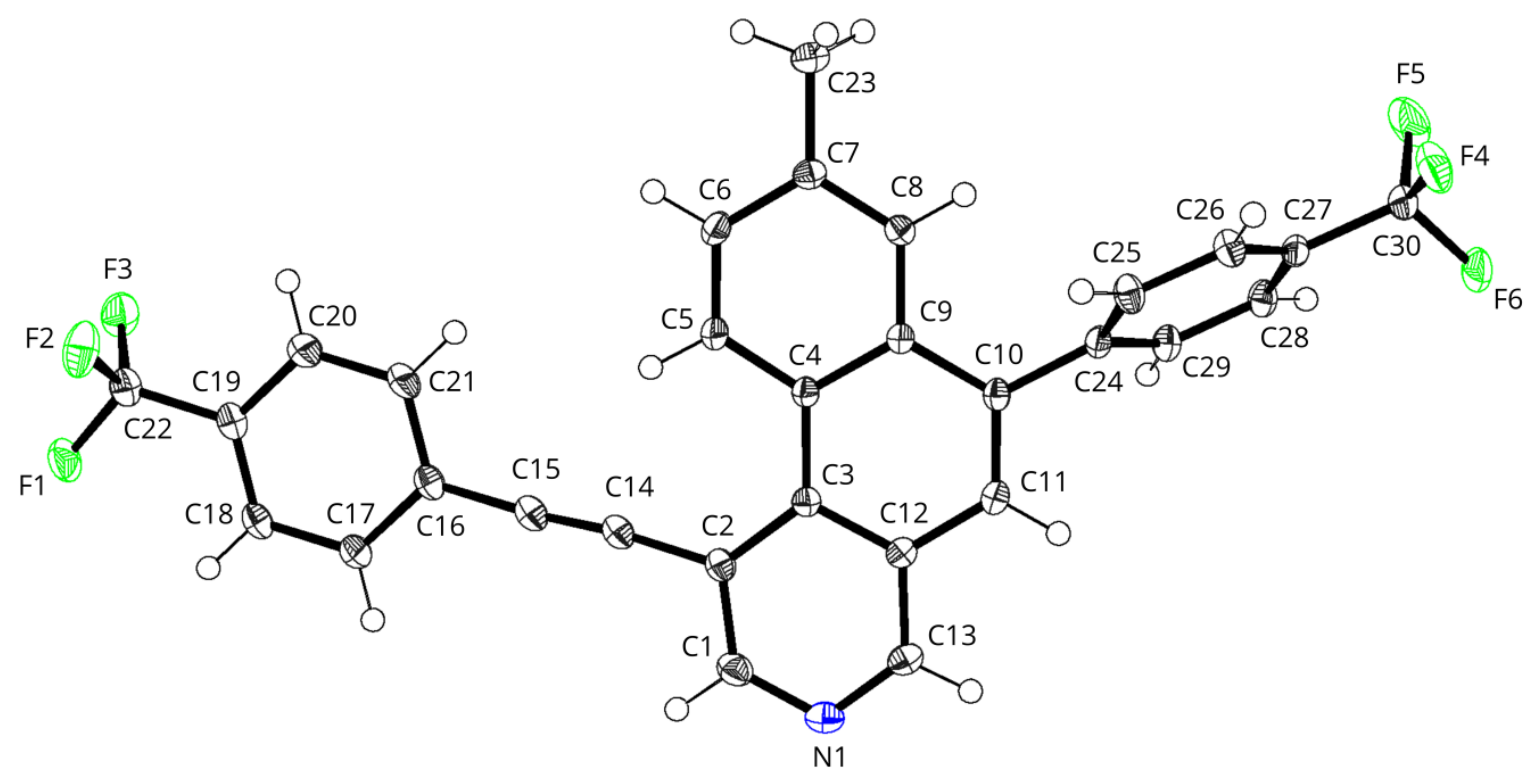

Figure S2. ORTEP diagram of $\mathbf{4 j}$ (determined from X-ray structural analysis at $123 \mathrm{~K}$ ). The thermal ellipsoids are drawn at the $50 \%$ probability level.

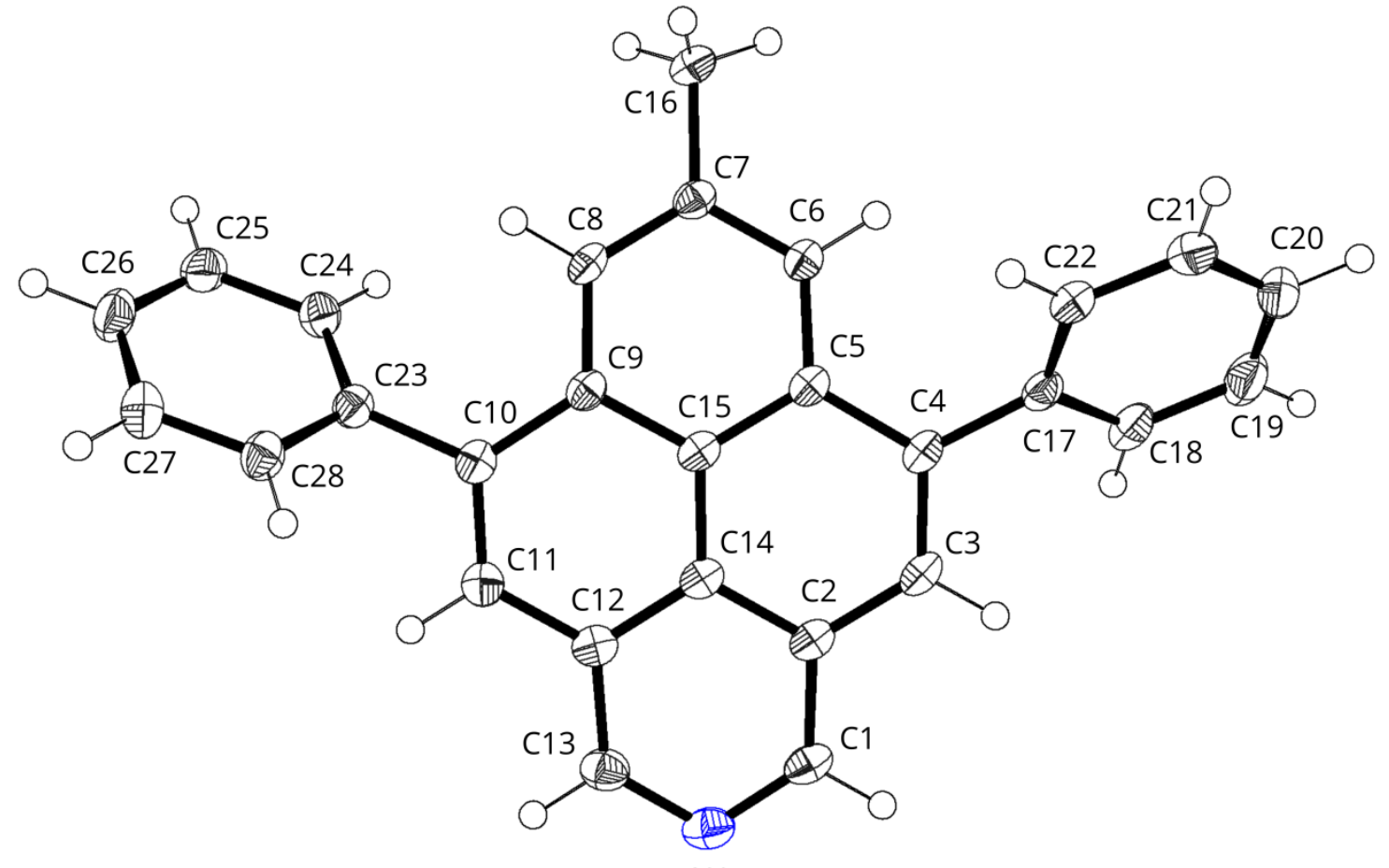

N1

Figure S3. ORTEP diagram of $\mathbf{5 b}$ (determined from X-ray structural analysis at $123 \mathrm{~K}$ ). The thermal ellipsoids are drawn at the $50 \%$ probability level. 
Table S3. Single crystal X-ray diffraction data for $\mathbf{5 b}$ and $\mathbf{4 j}$.

\begin{tabular}{|c|c|c|}
\hline & $5 b$ & $4 j$ \\
\hline Chem. Formula & $\mathrm{C}_{22} \mathrm{H}_{11} \mathrm{~N}$ & $\mathrm{C}_{30} \mathrm{H}_{17} \mathrm{~F}_{6} \mathrm{~N}$ \\
\hline Form. Wght $\left[\mathrm{g} \mathrm{mol}^{-1}\right]$ & 369.44 & 505.45 \\
\hline Crsyt. system & orthorhombic & monoclinic \\
\hline Space group (Hall group) & Pccn (-P 2ab 2ac) & $P 2_{1} / c(-P 2 y b c)$ \\
\hline$a[\AA ̊]$ & $31.461(5)$ & $27.2097(10)$ \\
\hline$b[\AA]$ & $32.311(4)$ & $4.6842(1)$ \\
\hline$c[\AA]$ & $7.4331(9)$ & $18.3065(7)$ \\
\hline$\alpha\left[^{\circ}\right]$ & 90 & 90 \\
\hline$\beta\left[^{\circ}\right]$ & 90 & $105.415(1)$ \\
\hline$\gamma\left[\left[^{\circ}\right]\right.$ & 90 & 90 \\
\hline$V\left[\AA^{3}\right]$ & $7556.2(17)$ & $2249.33(13)$ \\
\hline Z & 16 & 4 \\
\hline$N_{\text {ref }}$ & 9091 & 8104 \\
\hline$\theta_{\max }\left[{ }^{\circ}\right]$ & 27.999 & 32.499 \\
\hline$h, k, l_{\max }$ & $41,42,9$ & $41,7,27$ \\
\hline$\rho_{\mathrm{x}}\left[\mathrm{g} \mathrm{cm}^{-3}\right]$ & 1.299 & 1.493 \\
\hline$\mu\left[\mathrm{mm}^{-1}\right]$ & 0.075 & 0.122 \\
\hline 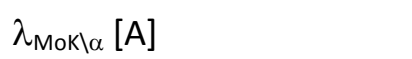 & 0.71073 & 0.71073 \\
\hline $\mathrm{T}[\mathrm{K}]$ & 123 & 123 \\
\hline$F(000)$ & 3104.0 & 1032.0 \\
\hline$N_{\text {par }}$ & 526 & 335 \\
\hline $\mathrm{R}$ & $0.0461(6423)$ & $0.0454(5856)$ \\
\hline $\mathrm{w} R_{2}$ & $0.1248(9091)$ & $0.1215(8104)$ \\
\hline$S$ & 1.020 & 1.016 \\
\hline
\end{tabular}

\section{DFT and TD-DFT Calculations}

\section{Cartesian coordinates of the optimized ground-state $\left(\mathrm{S}_{0}\right)$ and excited-state $\left(\mathrm{S}_{1}\right)$ structures}

Calculated by DFT at the B3LYP/6-31G(p) level of theory for ground state in gas phase and within IEFPCM of dichloromethane and TD-DFT B3LYP/6-31+G(p) for excited state within IEFPCM of dichloromethane. The local minima of the optimized geometries were confirmed by frequency calculations, ensuring that no imaginary frequencies are presented. The stated Energie $E$ for the $S_{0}$ state is the sum of electronic and zero-point Energies. 


\begin{tabular}{|c|c|c|c|}
\hline \multicolumn{4}{|c|}{$\begin{array}{l}S_{0}: \text { pyrene } \\
E=-615.569437 \text { Hartree }\end{array}$} \\
\hline Symbol & $x$ & $\mathrm{Y}$ & $\bar{Z}$ \\
\hline $\mathrm{C}$ & 3.5250992 & 0.0000003 & 0.0003689 \\
\hline C & 2.8341708 & -1.2115378 & 0.0003071 \\
\hline C & 1.4295297 & -1.2369007 & 0.0001578 \\
\hline C & 0.7136349 & 0.0000001 & 0.0000718 \\
\hline C & 1.4295294 & 1.2369020 & 0.0001328 \\
\hline $\mathrm{C}$ & 2.8341683 & 1.2115393 & 0.0002805 \\
\hline$C$ & 0.6811512 & -2.4654282 & 0.0000913 \\
\hline C & -0.7136349 & -0.0000001 & -0.0000744 \\
\hline C & -1.4295279 & -1.2369014 & -0.0001370 \\
\hline C & -0.6811485 & -2.4654286 & -0.0000505 \\
\hline C & -2.8341692 & -1.2115394 & -0.0002801 \\
\hline $\mathrm{H}$ & -3.3812546 & -2.1510292 & -0.0003272 \\
\hline C & -3.5250991 & -0.0000024 & -0.0003592 \\
\hline C & -2.8341697 & 1.2115376 & -0.0002999 \\
\hline C & -1.4295311 & 1.2369013 & -0.0001577 \\
\hline C & -0.6811506 & 2.4654291 & -0.0000950 \\
\hline$C$ & 0.6811480 & 2.4654294 & 0.0000427 \\
\hline $\mathrm{H}$ & 1.2305128 & 3.4037127 & 0.0000833 \\
\hline $\mathrm{H}$ & -1.2305171 & 3.4037117 & -0.0001579 \\
\hline $\mathrm{H}$ & 1.2305153 & -3.4037120 & 0.0001542 \\
\hline $\mathrm{H}$ & 4.6116456 & 0.0000019 & 0.0004853 \\
\hline $\mathrm{H}$ & 3.3812573 & -2.1510269 & 0.0003769 \\
\hline $\mathrm{H}$ & 3.3812585 & 2.1510262 & 0.0003268 \\
\hline $\mathrm{H}$ & -1.2305145 & -3.4037113 & -0.0001026 \\
\hline $\mathrm{H}$ & -4.6116455 & -0.0000009 & -0.0004698 \\
\hline $\mathrm{H}$ & -3.3812590 & 2.1510252 & -0.0003634 \\
\hline
\end{tabular}

$\mathrm{S}_{0}$ (gas phase) : pyrene

$E=-615.565436$ Hartree

\begin{tabular}{llll}
\hline Symbol & $\mathrm{X}$ & $\mathrm{Y}$ & $\mathrm{Z}$ \\
\hline $\mathrm{C}$ & 3.5239425 & 0.0000004 & 0.0003681 \\
$\mathrm{C}$ & 2.8329849 & -1.2106217 & 0.0003065 \\
$\mathrm{C}$ & 1.4290989 & -1.2362579 & 0.0001579 \\
$\mathrm{C}$ & 0.7133015 & 0.0000001 & 0.0000722 \\
$\mathrm{C}$ & 1.4290984 & 1.2362592 & 0.0001335 \\
$\mathrm{C}$ & 2.8329823 & 1.2106233 & 0.0002804 \\
\hline
\end{tabular}




\begin{tabular}{llll}
\hline $\mathrm{C}$ & 0.6807966 & -2.4638612 & 0.0000916 \\
$\mathrm{C}$ & -0.7133016 & -0.0000001 & -0.0000742 \\
$\mathrm{C}$ & -1.4290970 & -1.2362586 & -0.0001368 \\
$\mathrm{C}$ & -0.6807938 & -2.4638617 & -0.0000501 \\
$\mathrm{C}$ & -2.8329831 & -1.2106234 & -0.0002803 \\
$\mathrm{H}$ & -3.3798063 & -2.1505036 & -0.0003277 \\
$\mathrm{C}$ & -3.5239424 & -0.0000025 & -0.0003595 \\
$\mathrm{C}$ & -2.8329837 & 1.2106215 & -0.0003001 \\
$\mathrm{C}$ & -1.4291003 & 1.2362584 & -0.0001575 \\
$\mathrm{C}$ & -0.6807961 & 2.4638621 & -0.0000949 \\
$\mathrm{C}$ & 0.6807932 & 2.4638624 & 0.0000431 \\
$\mathrm{H}$ & 1.2304012 & 3.4022196 & 0.0000837 \\
$\mathrm{H}$ & -1.2304058 & 3.4022185 & -0.0001585 \\
$\mathrm{H}$ & 1.2304040 & -3.4022188 & 0.0001544 \\
$\mathrm{H}$ & 4.6106470 & 0.0000019 & 0.0004838 \\
$\mathrm{H}$ & 3.3798092 & -2.1505012 & 0.0003757 \\
$\mathrm{H}$ & 3.3798102 & 2.1505007 & 0.0003261 \\
$\mathrm{H}$ & -1.2304029 & -3.4022182 & -0.0001027 \\
$\mathrm{H}$ & -4.6106467 & -0.0000011 & -0.0004706 \\
$\mathrm{H}$ & -3.3798108 & 2.1504995 & -0.0003641 \\
\hline
\end{tabular}

\section{$\mathrm{S}_{0}$ : 2-azapyrene}

$E=-631.618414$ Hartree

\begin{tabular}{llll}
\hline Symbol & $\mathrm{X}$ & $\mathrm{Y}$ & $\mathrm{Z}$ \\
\hline $\mathrm{C}$ & 2.8351334 & -1.1480697 & 0.0003221 \\
$\mathrm{C}$ & 1.4332201 & -1.2249246 & 0.0001630 \\
$\mathrm{C}$ & 0.7100862 & -0.0000003 & 0.0000669 \\
$\mathrm{C}$ & 1.4332247 & 1.2249244 & 0.0001281 \\
$\mathrm{C}$ & 2.8351317 & 1.1480699 & 0.0002783 \\
$\mathrm{C}$ & 0.7019259 & -2.4625262 & 0.0000970 \\
$\mathrm{C}$ & -0.7128172 & 0.0000000 & -0.0000805 \\
$\mathrm{C}$ & -1.4188972 & -1.2402312 & -0.0001395 \\
C & -0.6611675 & -2.4660291 & -0.0000463 \\
$\mathrm{C}$ & -2.8231680 & -1.2143933 & -0.0002825 \\
$\mathrm{H}$ & -3.3735898 & -2.1515979 & -0.0003254 \\
C & -3.5106876 & -0.0000026 & -0.0003674 \\
C & -2.8231649 & 1.2143943 & -0.0003076 \\
C & -1.4189009 & 1.2402337 & -0.0001635 \\
C & -0.6611649 & 2.4660311 & -0.0000913 \\
C & 0.7019259 & 2.4625273 & 0.0000503 \\
\hline
\end{tabular}




\begin{tabular}{llll}
\hline $\mathrm{H}$ & -1.2069273 & 3.4061135 & -0.0001505 \\
$\mathrm{H}$ & 1.2574261 & -3.3966803 & 0.0001700 \\
$\mathrm{H}$ & 3.4239571 & -2.0648908 & 0.0004088 \\
$\mathrm{H}$ & 3.4239634 & 2.0648852 & 0.0003697 \\
$\mathrm{H}$ & -1.2069304 & -3.4061110 & -0.0000891 \\
$\mathrm{H}$ & -4.5970664 & 0.0000024 & -0.0004780 \\
$\mathrm{H}$ & -3.3735954 & 2.1515938 & -0.0003709 \\
$\mathrm{~N}$ & 3.5244652 & -0.0000026 & 0.0003724 \\
\hline
\end{tabular}

$\mathrm{S}_{0}$ (gase phase): 2-azapyrene

$E=-631.612954$ Hartree

\begin{tabular}{llll}
\hline Symbol & $\mathrm{X}$ & $\mathrm{Y}$ & $\mathrm{Z}$ \\
\hline $\mathrm{C}$ & 2.8353408 & -1.1458745 & 0.0003257 \\
$\mathrm{C}$ & 1.4333460 & -1.2241064 & 0.0001622 \\
$\mathrm{C}$ & 0.7098100 & -0.0000003 & 0.0000646 \\
$\mathrm{C}$ & 1.4333505 & 1.2241063 & 0.0001271 \\
$\mathrm{C}$ & 2.8353391 & 1.1458747 & 0.0002782 \\
$\mathrm{C}$ & 0.7014236 & -2.4604959 & 0.0000953 \\
$\mathrm{C}$ & -0.7129848 & 0.0000000 & -0.0000817 \\
$\mathrm{C}$ & -1.4191728 & -1.2397467 & -0.0001405 \\
$\mathrm{C}$ & -0.6611366 & -2.4646518 & -0.0000480 \\
$\mathrm{C}$ & -2.8228986 & -1.2135184 & -0.0002820 \\
$\mathrm{H}$ & -3.3731055 & -2.1511867 & -0.0003249 \\
$\mathrm{C}$ & -3.5104835 & -0.0000026 & -0.0003651 \\
$\mathrm{C}$ & -2.8228956 & 1.2135194 & -0.0003052 \\
$\mathrm{C}$ & -1.4191765 & 1.2397491 & -0.0001632 \\
$\mathrm{C}$ & -0.6611340 & 2.4646538 & -0.0000929 \\
$\mathrm{C}$ & 0.7014236 & 2.4604970 & 0.0000484 \\
$\mathrm{H}$ & 1.2573681 & 3.3947111 & 0.0000897 \\
$\mathrm{H}$ & -1.2070430 & 3.4049649 & -0.0001521 \\
$\mathrm{H}$ & 1.2573669 & -3.3947107 & 0.0001666 \\
$\mathrm{H}$ & 3.4264735 & -2.0617207 & 0.0004139 \\
$\mathrm{H}$ & 3.4264799 & 2.0617150 & 0.0003713 \\
$\mathrm{H}$ & -1.2070461 & -3.4049624 & -0.0000937 \\
$\mathrm{H}$ & -4.5971025 & 0.0000023 & -0.0004758 \\
$\mathrm{H}$ & -3.3731110 & 2.1511826 & -0.0003679 \\
$\mathrm{~N}$ & 3.5241162 & -0.0000026 & 0.0003765 \\
\hline & & & \\
\hline & & &
\end{tabular}




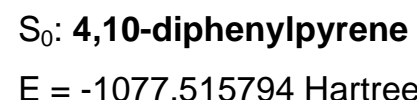

\begin{tabular}{|c|c|c|c|}
\hline Symbol & $X$ & $\bar{Y}$ & $Z$ \\
\hline $\mathrm{C}$ & -0.0000158 & -4.3896591 & 0.0000262 \\
\hline C & -1.2099303 & -3.6977111 & 0.0321811 \\
\hline C & -1.2324710 & -2.2924432 & 0.0324608 \\
\hline C & -0.0000127 & -1.5753648 & 0.0000124 \\
\hline C & 1.2324527 & -2.2924465 & -0.0324470 \\
\hline C & 1.2099031 & -3.6977147 & -0.0321441 \\
\hline C & -2.4576470 & -1.5502579 & 0.0436276 \\
\hline C & -0.0000046 & -0.1481213 & 0.0000593 \\
\hline C & -1.2437533 & 0.5677905 & 0.0061339 \\
\hline C & -2.4918945 & -0.1820416 & 0.0135282 \\
\hline C & -1.2102527 & 1.9724645 & 0.0309285 \\
\hline $\mathrm{H}$ & -2.1394141 & 2.5289237 & 0.0710984 \\
\hline C & -0.0000090 & 2.6596368 & 0.0002969 \\
\hline C & 1.2102405 & 1.9724628 & -0.0304785 \\
\hline C & 1.2437585 & 0.5677885 & -0.0059421 \\
\hline C & 2.4918927 & -0.1820501 & -0.0134990 \\
\hline C & 2.4576252 & -1.5502709 & -0.0436378 \\
\hline $\mathrm{H}$ & 3.3913929 & -2.1070688 & -0.0425645 \\
\hline $\mathrm{H}$ & -3.3914112 & -2.1070598 & 0.0424976 \\
\hline $\mathrm{H}$ & -0.0000204 & -5.4761508 & 0.0000413 \\
\hline $\mathrm{H}$ & -2.1504165 & -4.2423549 & 0.0552489 \\
\hline $\mathrm{H}$ & 2.1503877 & -4.2423608 & -0.0552011 \\
\hline $\mathrm{H}$ & 0.0000084 & 3.7461777 & 0.0004222 \\
\hline $\mathrm{H}$ & 2.1393807 & 2.5289602 & -0.0705808 \\
\hline C & 3.8178692 & 0.5021850 & 0.0136415 \\
\hline C & 4.1745516 & 1.3717390 & 1.0592835 \\
\hline C & 4.7665304 & 0.2399047 & -0.9882790 \\
\hline C & 5.4387723 & 1.9606461 & 1.0985608 \\
\hline $\mathrm{H}$ & 3.4620459 & 1.5732634 & 1.8543307 \\
\hline C & 6.0308361 & 0.8306156 & -0.9504356 \\
\hline $\mathrm{H}$ & 4.5028617 & -0.4247571 & -1.8064274 \\
\hline C & 6.3712332 & 1.6939135 & 0.0928220 \\
\hline $\mathrm{H}$ & 5.6972665 & 2.6236949 & 1.9198983 \\
\hline $\mathrm{H}$ & 6.7478910 & 0.6178336 & -1.7388759 \\
\hline $\mathrm{H}$ & 7.3548200 & 2.1546516 & 0.1230920 \\
\hline C & -3.8178556 & 0.5022228 & -0.0136900 \\
\hline C & -4.1743912 & 1.3718285 & -1.0593157 \\
\hline C & -4.7666521 & 0.2398734 & 0.9881027 \\
\hline
\end{tabular}




\begin{tabular}{llll}
\hline $\mathrm{C}$ & -5.4386260 & 1.9607411 & -1.0987309 \\
$\mathrm{H}$ & -3.4617874 & 1.5734312 & -1.8542521 \\
$\mathrm{C}$ & -6.0309413 & 0.8305730 & 0.9501182 \\
$\mathrm{H}$ & -4.5030729 & -0.4248430 & 1.8062360 \\
$\mathrm{C}$ & -6.3712077 & 1.6939367 & -0.0931509 \\
$\mathrm{H}$ & -5.6969946 & 2.6238205 & -1.9200836 \\
$\mathrm{H}$ & -6.7481297 & 0.6177581 & 1.7384272 \\
$\mathrm{H}$ & -7.3548128 & 2.1546319 & -0.1235059 \\
\hline
\end{tabular}

$\mathrm{S}_{0}: \mathbf{5 a}$

$E=-1093.564855$ Hartree

\begin{tabular}{llll}
\hline Symbol & $\mathrm{X}$ & $\mathrm{Y}$ & $\mathrm{Z}$ \\
\hline $\mathrm{C}$ & -1.1466528 & -3.6881389 & 0.0275036 \\
$\mathrm{C}$ & -1.2207512 & -2.2851354 & 0.0298247 \\
$\mathrm{C}$ & -0.0000065 & -1.5609830 & -0.0000741 \\
$\mathrm{C}$ & 1.2206934 & -2.2852376 & -0.0298546 \\
$\mathrm{C}$ & 1.1465427 & -3.6881856 & -0.0274040 \\
$\mathrm{C}$ & -2.4546486 & -1.5602392 & 0.0399317 \\
$\mathrm{C}$ & 0.0000344 & -0.1385230 & -0.0001700 \\
$\mathrm{C}$ & -1.2469626 & 0.5677810 & 0.0050738 \\
$\mathrm{C}$ & -2.4926597 & -0.1909164 & 0.0111280 \\
$\mathrm{C}$ & -1.2129426 & 1.9721771 & 0.0295008 \\
$\mathrm{H}$ & -2.1403259 & 2.5314237 & 0.0686212 \\
$\mathrm{C}$ & 0.0000920 & 2.6561256 & -0.0005542 \\
$\mathrm{C}$ & 1.2131308 & 1.9720793 & -0.0303758 \\
$\mathrm{C}$ & 1.2470707 & 0.5677546 & -0.0055757 \\
$\mathrm{C}$ & 2.4927493 & -0.1910569 & -0.0114467 \\
$\mathrm{C}$ & 2.4546465 & -1.5603545 & -0.0399886 \\
$\mathrm{H}$ & 3.3845684 & -2.1230444 & -0.0372605 \\
$\mathrm{H}$ & -3.3846137 & -2.1228563 & 0.0374687 \\
$\mathrm{H}$ & -2.0646000 & -4.2744461 & 0.0479248 \\
$\mathrm{H}$ & 2.0644266 & -4.2745845 & -0.0476722 \\
$\mathrm{H}$ & 0.0001522 & 3.7425433 & -0.0007294 \\
$\mathrm{H}$ & 2.1405329 & 2.5312994 & -0.0696247 \\
$\mathrm{~N}$ & -0.0000986 & -4.3784081 & 0.0000887 \\
$\mathrm{C}$ & 3.8200126 & 0.4901480 & 0.0145453 \\
$\mathrm{C}$ & 4.1762101 & 1.3631596 & 1.0573339 \\
$\mathrm{C}$ & 4.7682767 & 0.2218369 & -0.9859833 \\
$\mathrm{C}$ & 5.4413146 & 1.9501293 & 1.0950611 \\
$\mathrm{H}$ & 3.4638370 & 1.5681974 & 1.8515681 \\
\hline & & & \\
\hline & & &
\end{tabular}




\begin{tabular}{llll}
\hline $\mathrm{C}$ & 6.0330803 & 0.8113222 & -0.9495732 \\
$\mathrm{H}$ & 4.5042731 & -0.4455133 & -1.8017804 \\
$\mathrm{C}$ & 6.3736871 & 1.6781063 & 0.0907177 \\
$\mathrm{H}$ & 5.7004290 & 2.6157612 & 1.9140067 \\
$\mathrm{H}$ & 6.7503659 & 0.5946108 & -1.7366157 \\
$\mathrm{H}$ & 7.3578670 & 2.1375351 & 0.1198390 \\
$\mathrm{C}$ & -3.8199464 & 0.4902905 & -0.0146102 \\
$\mathrm{C}$ & -4.1767566 & 1.3627286 & -1.0575440 \\
$\mathrm{C}$ & -4.7677623 & 0.2222140 & 0.9865159 \\
$\mathrm{C}$ & -5.4419829 & 1.9496424 & -1.0948519 \\
$\mathrm{H}$ & -3.4649263 & 1.5675096 & -1.8523312 \\
$\mathrm{C}$ & -6.0325573 & 0.8115853 & 0.9505314 \\
$\mathrm{H}$ & -4.5032475 & -0.4449129 & 1.8023282 \\
$\mathrm{C}$ & -6.3737609 & 1.6780165 & -0.0899431 \\
$\mathrm{H}$ & -5.7014682 & 2.6148798 & -1.9139987 \\
$\mathrm{H}$ & -6.7494731 & 0.5951787 & 1.7379971 \\
$\mathrm{H}$ & -7.3580122 & 2.1373161 & -0.1186748 \\
\hline
\end{tabular}

$\mathrm{S}_{0}$ (gase phase): $\mathbf{5 a}$

$E=-1093.557757$ Hartree

\begin{tabular}{llll}
\hline Symbol & $\mathrm{X}$ & $\mathrm{Y}$ & $\mathrm{Z}$ \\
\hline $\mathrm{C}$ & -1.1444727 & -3.6895741 & 0.0273605 \\
$\mathrm{C}$ & -1.2202055 & -2.2864830 & 0.0300403 \\
$\mathrm{C}$ & -0.0000047 & -1.5619903 & -0.0000672 \\
$\mathrm{C}$ & 1.2201677 & -2.2865566 & -0.0300541 \\
$\mathrm{C}$ & 1.1444634 & -3.6895839 & -0.0272876 \\
$\mathrm{C}$ & -2.4531223 & -1.5606589 & 0.0406823 \\
$\mathrm{C}$ & 0.0000200 & -0.1395237 & -0.0001648 \\
$\mathrm{C}$ & -1.2463810 & 0.5669076 & 0.0061704 \\
$\mathrm{C}$ & -2.4914231 & -0.1917079 & 0.0126617 \\
$\mathrm{C}$ & -1.2127957 & 1.9710699 & 0.0307246 \\
$\mathrm{H}$ & -2.1414305 & 2.5284225 & 0.0724401 \\
$\mathrm{C}$ & -0.0000049 & 2.6545296 & -0.0005185 \\
$\mathrm{C}$ & 1.2128288 & 1.9710216 & -0.0315449 \\
$\mathrm{C}$ & 1.2464168 & 0.5669641 & -0.0066496 \\
$\mathrm{C}$ & 2.4914632 & -0.1917150 & -0.0129274 \\
$\mathrm{C}$ & 2.4530942 & -1.5606511 & -0.0406788 \\
$\mathrm{H}$ & 3.3841926 & -2.1218765 & -0.0357678 \\
$\mathrm{H}$ & -3.3842400 & -2.1218528 & 0.0360386 \\
$\mathrm{H}$ & -2.0615321 & -4.2781068 & 0.0472426 \\
\hline & & & \\
\hline
\end{tabular}




\begin{tabular}{llll}
\hline $\mathrm{H}$ & 2.0614726 & -4.2781827 & -0.0470818 \\
$\mathrm{H}$ & 0.0000360 & 3.7412905 & -0.0006721 \\
$\mathrm{H}$ & 2.1414594 & 2.5284036 & -0.0733480 \\
$\mathrm{~N}$ & -0.0000415 & -4.3793574 & 0.0000510 \\
$\mathrm{C}$ & 3.8180603 & 0.4899906 & 0.0136951 \\
$\mathrm{C}$ & 4.1773824 & 1.3536594 & 1.0625237 \\
$\mathrm{C}$ & 4.7627069 & 0.2332825 & -0.9923570 \\
$\mathrm{C}$ & 5.4412355 & 1.9417312 & 1.1009996 \\
$\mathrm{H}$ & 3.4663831 & 1.5486985 & 1.8606335 \\
$\mathrm{C}$ & 6.0265452 & 0.8234584 & -0.9550930 \\
$\mathrm{H}$ & 4.4943627 & -0.4257331 & -1.8135858 \\
$\mathrm{C}$ & 6.3699396 & 1.6803622 & 0.0913514 \\
$\mathrm{H}$ & 5.7031331 & 2.5997396 & 1.9254569 \\
$\mathrm{H}$ & 6.7412773 & 0.6153199 & -1.7469762 \\
$\mathrm{H}$ & 7.3538536 & 2.1406209 & 0.1212300 \\
$\mathrm{C}$ & -3.8180092 & 0.4900704 & -0.0137147 \\
$\mathrm{C}$ & -4.1777982 & 1.3532175 & -1.0626588 \\
$\mathrm{C}$ & -4.7623077 & 0.2335419 & 0.9928299 \\
$\mathrm{C}$ & -5.4417438 & 1.9413349 & -1.1007910 \\
$\mathrm{H}$ & -3.4672664 & 1.5479867 & -1.8612554 \\
$\mathrm{C}$ & -6.0260707 & 0.8237058 & 0.9559282 \\
$\mathrm{H}$ & -4.4935480 & -0.4253736 & 1.8139999 \\
$\mathrm{C}$ & -6.3699182 & 1.6803788 & -0.0906671 \\
$\mathrm{H}$ & -5.7038846 & 2.5989990 & -1.9254433 \\
$\mathrm{H}$ & -6.7405068 & 0.6158775 & 1.7481623 \\
$\mathrm{H}$ & -7.3538694 & 2.1405787 & -0.1201893 \\
\hline & & & \\
\hline & & & \\
$\mathrm{H}$ & & &
\end{tabular}

$\mathrm{S}_{0}: 5 b$

$E=-1132.855890$ Hartree

\begin{tabular}{llll}
\hline Symbol & $\mathrm{X}$ & $\mathrm{Y}$ & $\mathrm{Z}$ \\
\hline $\mathrm{C}$ & 1.1471680 & -3.8698860 & 0.1626855 \\
$\mathrm{C}$ & 1.2214049 & -2.4684835 & 0.0997072 \\
$\mathrm{C}$ & 0.0000133 & -1.7454820 & 0.0714030 \\
$\mathrm{C}$ & -1.2213897 & -2.4684718 & 0.0997739 \\
$\mathrm{C}$ & -1.1471510 & -3.8698721 & 0.1627721 \\
$\mathrm{C}$ & 2.4540285 & -1.7411472 & 0.0753370 \\
$\mathrm{C}$ & 0.0000175 & -0.3260830 & -0.0019080 \\
$\mathrm{C}$ & 1.2429965 & 0.3818399 & -0.0563472 \\
$\mathrm{C}$ & 2.4902323 & -0.3725396 & 0.0134519 \\
$\mathrm{C}$ & 1.2064439 & 1.7755567 & -0.2197730 \\
\hline
\end{tabular}




\begin{tabular}{|c|c|c|c|}
\hline $\mathrm{H}$ & 2.1380403 & 2.3250892 & -0.3004648 \\
\hline C & 0.0000129 & 2.4772468 & -0.3051307 \\
\hline C & -1.2064347 & 1.7755474 & -0.2197259 \\
\hline C & -1.2429778 & 0.3818424 & -0.0563116 \\
\hline C & -2.4902173 & -0.3725328 & 0.0135174 \\
\hline C & -2.4540139 & -1.7411408 & 0.0754346 \\
\hline $\mathrm{H}$ & -3.3846346 & -2.2997881 & 0.1327192 \\
\hline $\mathrm{H}$ & 3.3846486 & -2.2998022 & 0.1325773 \\
\hline $\mathrm{H}$ & 2.0652155 & -4.4559568 & 0.1879085 \\
\hline $\mathrm{H}$ & -2.0652012 & -4.4559379 & 0.1880347 \\
\hline $\mathrm{H}$ & -2.1380336 & 2.3250857 & -0.3003759 \\
\hline $\mathrm{N}$ & 0.0000071 & -4.5595608 & 0.1937294 \\
\hline C & -3.8157387 & 0.3116919 & 0.0173440 \\
\hline C & -4.7916539 & -0.0378500 & -0.9304181 \\
\hline C & -4.1430162 & 1.2710980 & 0.9915181 \\
\hline C & -6.0548550 & 0.5556338 & -0.9092795 \\
\hline $\mathrm{H}$ & -4.5501143 & -0.7720205 & -1.6940947 \\
\hline C & -5.4064301 & 1.8624055 & 1.0141831 \\
\hline $\mathrm{H}$ & -3.4087756 & 1.5404727 & 1.7454819 \\
\hline C & -6.3663878 & 1.5084033 & 0.0627428 \\
\hline $\mathrm{H}$ & -6.7936120 & 0.2752221 & -1.6552810 \\
\hline $\mathrm{H}$ & -5.6427060 & 2.5958418 & 1.7804437 \\
\hline $\mathrm{H}$ & -7.3493077 & 1.9711603 & 0.0798673 \\
\hline C & 3.8157543 & 0.3116886 & 0.0172843 \\
\hline C & 4.1429840 & 1.2710690 & 0.9915260 \\
\hline C & 4.7916885 & -0.0377914 & -0.9304524 \\
\hline C & 5.4063766 & 1.8623898 & 1.0142584 \\
\hline $\mathrm{H}$ & 3.4086979 & 1.5403864 & 1.7454647 \\
\hline C & 6.0548912 & 0.5557175 & -0.9092467 \\
\hline $\mathrm{H}$ & 4.5502002 & -0.7719261 & -1.6941805 \\
\hline C & 6.3663756 & 1.5084371 & 0.0628237 \\
\hline $\mathrm{H}$ & 5.6426403 & 2.5957984 & 1.7805492 \\
\hline $\mathrm{H}$ & 6.7936779 & 0.2753417 & -1.6552318 \\
\hline $\mathrm{H}$ & 7.3492865 & 1.9712121 & 0.0800055 \\
\hline C & -0.0000671 & 3.9792768 & -0.4715608 \\
\hline $\mathrm{H}$ & -0.8862908 & 4.3210015 & -1.0158537 \\
\hline $\mathrm{H}$ & -0.0026123 & 4.4827781 & 0.5042890 \\
\hline $\mathrm{H}$ & 0.8885020 & 4.3215822 & -1.0116192 \\
\hline
\end{tabular}




\section{$\mathrm{S}_{0}: 5 \mathrm{c}$}

$E=-1208.057021$ Hartree

\begin{tabular}{|c|c|c|c|}
\hline Symbol & $\bar{X}$ & $\bar{Y}$ & $Z$ \\
\hline C & 0.9801583 & 4.0796115 & -0.1028700 \\
\hline C & 1.0954067 & 2.6795245 & -0.0697234 \\
\hline C & -0.1036038 & 1.9197979 & -0.0536647 \\
\hline C & -1.3455413 & 2.6084130 & -0.0647206 \\
\hline C & -1.3124995 & 4.0110053 & -0.0982492 \\
\hline C & 2.3473907 & 1.9876416 & -0.0631156 \\
\hline C & -0.0619006 & 0.5013426 & -0.0098491 \\
\hline C & 1.1989914 & -0.1722302 & 0.0255693 \\
\hline C & 2.4231038 & 0.6192008 & -0.0312552 \\
\hline C & 1.2177241 & -1.5721473 & 0.1530817 \\
\hline $\mathrm{H}$ & 2.1678282 & -2.0846283 & 0.2113371 \\
\hline C & 0.0221582 & -2.2905303 & 0.2256447 \\
\hline C & -1.2144313 & -1.6352123 & 0.1597530 \\
\hline C & -1.2856464 & -0.2457855 & 0.0314201 \\
\hline C & -2.5555136 & 0.4748570 & -0.0214181 \\
\hline C & -2.5570199 & 1.8440295 & -0.0538437 \\
\hline $\mathrm{H}$ & -3.5034207 & 2.3769179 & -0.0972605 \\
\hline $\mathrm{H}$ & 3.2621681 & 2.5729662 & -0.1084403 \\
\hline $\mathrm{H}$ & 1.8812271 & 4.6919825 & -0.1176750 \\
\hline $\mathrm{H}$ & -2.2473426 & 4.5704090 & -0.1096074 \\
\hline $\mathrm{H}$ & -2.1126639 & -2.2368211 & 0.2260213 \\
\hline$N$ & -0.1856340 & 4.7362710 & -0.1169937 \\
\hline C & -3.8595626 & -0.2486632 & -0.0355201 \\
\hline C & -4.8429228 & 0.0528133 & 0.9209878 \\
\hline C & -4.1601057 & -1.1995072 & -1.0266366 \\
\hline C & -6.0875807 & -0.5783233 & 0.8914546 \\
\hline $\mathrm{H}$ & -4.6216218 & 0.7791977 & 1.6981265 \\
\hline C & -5.4049258 & -1.8285640 & -1.0574591 \\
\hline $\mathrm{H}$ & -3.4202673 & -1.4328191 & -1.7870703 \\
\hline C & -6.3726492 & -1.5218715 & -0.0975203 \\
\hline $\mathrm{H}$ & -6.8323100 & -0.3345578 & 1.6443649 \\
\hline $\mathrm{H}$ & -5.6206479 & -2.5547083 & -1.8366016 \\
\hline $\mathrm{H}$ & -7.3408877 & -2.0143328 & -0.1210043 \\
\hline C & 3.7681003 & -0.0263144 & -0.0496428 \\
\hline C & 4.1275700 & -0.9424855 & -1.0537481 \\
\hline C & 4.7283771 & 0.3180760 & 0.9156333 \\
\hline C & 5.4073000 & -1.4971660 & -1.0882416 \\
\hline $\mathrm{H}$ & 3.4053587 & -1.2062785 & -1.8213457 \\
\hline
\end{tabular}




\begin{tabular}{llll}
\hline $\mathrm{C}$ & 6.0080036 & -0.2387250 & 0.8824381 \\
$\mathrm{H}$ & 4.4622925 & 1.0194616 & 1.7016325 \\
$\mathrm{C}$ & 6.3515909 & -1.1488440 & -0.1191509 \\
$\mathrm{H}$ & 5.6686747 & -2.1967344 & -1.8776756 \\
$\mathrm{H}$ & 6.7346460 & 0.0371632 & 1.6418880 \\
$\mathrm{H}$ & 7.3473847 & -1.5827021 & -0.1457827 \\
$\mathrm{O}$ & -0.0485117 & -3.6435904 & 0.3671617 \\
$\mathrm{C}$ & 1.1652703 & -4.3867799 & 0.4505581 \\
$\mathrm{H}$ & 1.7598412 & -4.0862501 & 1.3215881 \\
$\mathrm{H}$ & 1.7674260 & -4.2721035 & -0.4586380 \\
$\mathrm{H}$ & 0.8663939 & -5.4303141 & 0.5583339 \\
\hline
\end{tabular}

$\mathrm{S}_{0}$ (gas phase): $\mathbf{5 c}$

$E=-1208.048437$ Hartree

\begin{tabular}{llll}
\hline Symbol & $\mathrm{X}$ & $\mathrm{Y}$ & $\mathrm{Z}$ \\
\hline $\mathrm{C}$ & 0.9757055 & 4.0823048 & 0.1004100 \\
$\mathrm{C}$ & 1.0936762 & 2.6821312 & 0.0671398 \\
$\mathrm{C}$ & -0.1041809 & 1.9211463 & 0.0513931 \\
$\mathrm{C}$ & -1.3461247 & 2.6089670 & 0.0630656 \\
$\mathrm{C}$ & -1.3125523 & 4.0117255 & 0.0968091 \\
$\mathrm{C}$ & 2.3452800 & 1.9905157 & 0.0601384 \\
$\mathrm{C}$ & -0.0616289 & 0.5025196 & 0.0082316 \\
$\mathrm{C}$ & 1.1991269 & -0.1695801 & -0.0266376 \\
$\mathrm{C}$ & 2.4217049 & 0.6224448 & 0.0289562 \\
$\mathrm{C}$ & 1.2190666 & -1.5699509 & -0.1516995 \\
$\mathrm{H}$ & 2.1710712 & -2.0792603 & -0.2111607 \\
$\mathrm{C}$ & 0.0245220 & -2.2885008 & -0.2234455 \\
$\mathrm{C}$ & -1.2124944 & -1.6355009 & -0.1589232 \\
$\mathrm{C}$ & -1.2842989 & -0.2460484 & -0.0323230 \\
$\mathrm{C}$ & -2.5540131 & 0.4738456 & 0.0201229 \\
$\mathrm{C}$ & -2.5559685 & 1.8426682 & 0.0526370 \\
$\mathrm{H}$ & -3.5037794 & 2.3733507 & 0.0986309 \\
$\mathrm{H}$ & 3.2608052 & 2.5751144 & 0.1070099 \\
$\mathrm{H}$ & 1.8753246 & 4.6976063 & 0.1152452 \\
$\mathrm{H}$ & -2.2469508 & 4.5726515 & 0.1089168 \\
$\mathrm{H}$ & -2.1092783 & -2.2389071 & -0.2274897 \\
$\mathrm{~N}$ & -0.1883757 & 4.7374257 & 0.1150057 \\
$\mathrm{C}$ & -3.8571133 & -0.2505687 & 0.0355596 \\
$\mathrm{C}$ & -4.1585566 & -1.1959194 & 1.0308514 \\
$\mathrm{C}$ & -4.8388463 & 0.0431507 & -0.9240821 \\
\hline & & & \\
\hline & & &
\end{tabular}




\begin{tabular}{llll}
\hline $\mathrm{C}$ & -5.4020032 & -1.8261593 & 1.0629671 \\
$\mathrm{H}$ & -3.4183774 & -1.4229442 & 1.7929056 \\
$\mathrm{C}$ & -6.0823947 & -0.5886978 & -0.8930505 \\
$\mathrm{H}$ & -4.6146970 & 0.7640563 & -1.7055831 \\
$\mathrm{C}$ & -6.3680919 & -1.5262887 & 0.1002755 \\
$\mathrm{H}$ & -5.6184069 & -2.5483329 & 1.8458540 \\
$\mathrm{H}$ & -6.8261062 & -0.3506872 & -1.6490499 \\
$\mathrm{C}$ & 3.7658942 & -0.0241348 & 0.0493319 \\
$\mathrm{C}$ & 4.7208502 & 0.2991264 & -0.9274802 \\
$\mathrm{C}$ & 4.1296873 & -0.9234266 & 1.0664432 \\
$\mathrm{C}$ & 5.9988141 & -0.2601462 & -0.8923360 \\
$\mathrm{H}$ & 4.4496400 & 0.9865184 & -1.7240937 \\
$\mathrm{C}$ & 5.4075070 & -1.4809353 & 1.1029175 \\
$\mathrm{H}$ & 3.4099566 & -1.1693750 & 1.8424666 \\
$\mathrm{C}$ & 6.3463906 & -1.1526997 & 0.1225198 \\
$\mathrm{H}$ & 6.7216959 & 0.0002448 & -1.6610376 \\
$\mathrm{H}$ & 5.6729202 & -2.1660488 & 1.9039512 \\
$\mathrm{H}$ & 7.3416008 & -1.5881592 & 0.1510427 \\
$\mathrm{H}$ & -7.3359213 & -2.0198230 & 0.1248228 \\
$\mathrm{C}$ & 1.1649898 & -4.3800713 & -0.4528573 \\
$\mathrm{H}$ & 0.8694624 & -5.4251272 & -0.5614737 \\
$\mathrm{H}$ & 1.7583871 & -4.0778086 & -1.3255450 \\
$\mathrm{H}$ & 1.7745724 & -4.2674348 & 0.4531932 \\
$\mathrm{O}$ & -0.0453719 & -3.6436394 & -0.3631567 \\
\hline & & & \\
\hline & & & \\
\hline & & &
\end{tabular}

$S_{0}: 5 d$

$E=-1430.597633$ Hartree

\begin{tabular}{llll}
\hline Symbol & $\mathrm{X}$ & $\mathrm{Y}$ & $\mathrm{Z}$ \\
\hline $\mathrm{C}$ & -1.1474274 & 4.3651511 & 0.0624298 \\
$\mathrm{C}$ & -1.2215567 & 2.9621022 & 0.0481388 \\
$\mathrm{C}$ & -0.0000234 & 2.2399181 & 0.0446702 \\
$\mathrm{C}$ & 1.2215067 & 2.9621146 & 0.0481175 \\
$\mathrm{C}$ & 1.1473675 & 4.3651571 & 0.0623960 \\
$\mathrm{C}$ & -2.4553542 & 2.2369146 & 0.0494664 \\
$\mathrm{C}$ & -0.0000172 & 0.8181922 & 0.0204003 \\
$\mathrm{C}$ & -1.2464642 & 0.1133652 & -0.0074180 \\
$\mathrm{C}$ & -2.4942403 & 0.8675254 & 0.0357741 \\
$\mathrm{C}$ & -1.2159109 & -1.2844327 & -0.1213950 \\
$\mathrm{H}$ & -2.1416791 & -1.8417978 & -0.1842361 \\
$\mathrm{C}$ & -0.0000100 & -1.9630021 & -0.1816823 \\
\hline
\end{tabular}




\begin{tabular}{|c|c|c|c|}
\hline$C$ & 1.2158940 & -1.2844137 & -0.1214296 \\
\hline C & 1.2464387 & 0.1133733 & -0.0074346 \\
\hline C & 2.4942139 & 0.8675473 & 0.0357575 \\
\hline C & 2.4553108 & 2.2369347 & 0.0494443 \\
\hline $\mathrm{H}$ & 3.3847686 & 2.7988161 & 0.0864100 \\
\hline $\mathrm{H}$ & -3.3848175 & 2.7987858 & 0.0864330 \\
\hline $\mathrm{H}$ & -2.0649584 & 4.9520083 & 0.0674558 \\
\hline $\mathrm{H}$ & 2.0648903 & 4.9520261 & 0.0674296 \\
\hline $\mathrm{H}$ & 2.1416653 & -1.8417747 & -0.1843066 \\
\hline $\mathrm{N}$ & -0.0000351 & 5.0540885 & 0.0694331 \\
\hline C & 3.8176071 & 0.1806844 & 0.0609894 \\
\hline C & 4.7915716 & 0.4951200 & -0.9008528 \\
\hline C & 4.1428591 & -0.7467309 & 1.0662859 \\
\hline C & 6.0521036 & -0.1027455 & -0.8630232 \\
\hline $\mathrm{H}$ & 4.5507698 & 1.2038053 & -1.6883965 \\
\hline C & 5.4038938 & -1.3422739 & 1.1049043 \\
\hline $\mathrm{H}$ & 3.4108969 & -0.9870222 & 1.8323440 \\
\hline C & 6.3621157 & -1.0240508 & 0.1394334 \\
\hline $\mathrm{H}$ & 6.7898495 & 0.1493426 & -1.6199166 \\
\hline $\mathrm{H}$ & 5.6392025 & -2.0508014 & 1.8943645 \\
\hline $\mathrm{H}$ & 7.3429900 & -1.4903240 & 0.1690593 \\
\hline C & -3.8176334 & 0.1806510 & 0.0610063 \\
\hline C & -4.1429400 & -0.7466554 & 1.0663773 \\
\hline C & -4.7915318 & 0.4949670 & -0.9009466 \\
\hline C & -5.4039731 & -1.3422136 & 1.1049715 \\
\hline $\mathrm{H}$ & -3.4110322 & -0.9868588 & 1.8325147 \\
\hline C & -6.0520552 & -0.1029129 & -0.8631424 \\
\hline $\mathrm{H}$ & -4.5506790 & 1.2035721 & -1.6885466 \\
\hline C & -6.3621269 & -1.0241110 & 0.1393982 \\
\hline $\mathrm{H}$ & -5.6393256 & -2.0506561 & 1.8944950 \\
\hline $\mathrm{H}$ & -6.7897546 & 0.1490747 & -1.6201147 \\
\hline $\mathrm{H}$ & -7.3429975 & -1.4903941 & 0.1689988 \\
\hline C & 0.0000501 & -3.4664992 & -0.2583379 \\
\hline $\mathrm{F}$ & 1.0889953 & -3.9441550 & -0.9020693 \\
\hline $\mathrm{F}$ & 0.0007638 & -4.0297808 & 0.9756727 \\
\hline $\mathrm{F}$ & -1.0894868 & -3.9443399 & -0.9009159 \\
\hline
\end{tabular}

$\mathrm{S}_{0}$ (gas phase): $\mathbf{5 d}$

$E=-1430.590314$ Hartree

\begin{tabular}{llll}
\hline Symbol & $X$ & $Y$ & $Z$ \\
\hline
\end{tabular}




\begin{tabular}{|c|c|c|c|}
\hline $\mathrm{C}$ & -1.1447029 & 4.3666136 & 0.0645878 \\
\hline C & -1.2206256 & 2.9634791 & 0.0484995 \\
\hline C & 0.0004215 & 2.2406195 & 0.0440863 \\
\hline C & 1.2215839 & 2.9632581 & 0.0484440 \\
\hline C & 1.1459108 & 4.3664125 & 0.0644595 \\
\hline C & -2.4534676 & 2.2372121 & 0.0490815 \\
\hline C & 0.0002703 & 0.8188090 & 0.0189618 \\
\hline C & -1.2457060 & 0.1135028 & -0.0093202 \\
\hline C & -2.4928612 & 0.8680963 & 0.0338076 \\
\hline C & -1.2152091 & -1.2843099 & -0.1226126 \\
\hline $\mathrm{H}$ & -2.1408422 & -1.8419090 & -0.1883227 \\
\hline C & 0.0000240 & -1.9638798 & -0.1817506 \\
\hline C & 1.2153237 & -1.2846460 & -0.1224499 \\
\hline C & 1.2460645 & 0.1132594 & -0.0092319 \\
\hline C & 2.4933348 & 0.8676337 & 0.0338725 \\
\hline C & 2.4542881 & 2.2367444 & 0.0490032 \\
\hline $\mathrm{H}$ & 3.3849969 & 2.7969635 & 0.0887652 \\
\hline $\mathrm{H}$ & -3.3840544 & 2.7976255 & 0.0889750 \\
\hline $\mathrm{H}$ & -2.0613005 & 4.9558359 & 0.0705962 \\
\hline $\mathrm{H}$ & 2.0626125 & 4.9554731 & 0.0705247 \\
\hline $\mathrm{H}$ & 2.1407928 & -1.8424700 & -0.1879289 \\
\hline$N$ & 0.0006705 & 5.0551120 & 0.0723397 \\
\hline C & 3.8163083 & 0.1805396 & 0.0591993 \\
\hline C & 4.7873623 & 0.4865973 & -0.9073247 \\
\hline C & 4.1440634 & -0.7404393 & 1.0688902 \\
\hline C & 6.0470101 & -0.1118067 & -0.8694544 \\
\hline $\mathrm{H}$ & 4.5426804 & 1.1888406 & -1.6995679 \\
\hline C & 5.4039314 & -1.3368412 & 1.1075435 \\
\hline $\mathrm{H}$ & 3.4128514 & -0.9742120 & 1.8377876 \\
\hline C & 6.3591357 & -1.0261125 & 0.1376048 \\
\hline $\mathrm{H}$ & 6.7825551 & 0.1337037 & -1.6308425 \\
\hline $\mathrm{H}$ & 5.6410624 & -2.0408927 & 1.9006314 \\
\hline $\mathrm{H}$ & 7.3395392 & -1.4936191 & 0.1670488 \\
\hline C & -3.8159500 & 0.1813058 & 0.0592459 \\
\hline C & -4.1438533 & -0.7397599 & 1.0688067 \\
\hline C & -4.7870457 & 0.4875817 & -0.9072287 \\
\hline C & -5.4038646 & -1.3358671 & 1.1074933 \\
\hline $\mathrm{H}$ & -3.4126295 & -0.9738873 & 1.8375908 \\
\hline C & -6.0468222 & -0.1104902 & -0.8693055 \\
\hline $\mathrm{H}$ & -4.5422292 & 1.1897352 & -1.6995115 \\
\hline C & -6.3591090 & -1.0247973 & 0.1377220 \\
\hline
\end{tabular}




\begin{tabular}{llll}
\hline $\mathrm{H}$ & -5.6410264 & -2.0399871 & 1.9005123 \\
$\mathrm{H}$ & -6.7823470 & 0.1352563 & -1.6306377 \\
$\mathrm{H}$ & -7.3396298 & -1.4920504 & 0.1672191 \\
$\mathrm{C}$ & -0.0008518 & -3.4689128 & -0.2543123 \\
$\mathrm{~F}$ & -0.0102735 & -4.0237671 & 0.9818796 \\
$\mathrm{~F}$ & 1.0927409 & -3.9461894 & -0.8882595 \\
$\mathrm{~F}$ & -1.0866350 & -3.9437103 & -0.9035234 \\
\hline
\end{tabular}

$\mathrm{S}_{0}: \mathbf{5 g}$

$E=-1172.146386$ Hartree

\begin{tabular}{llll}
\hline Symbol & $\mathrm{X}$ & $\mathrm{Y}$ & $\mathrm{Z}$ \\
\hline $\mathrm{C}$ & 1.2206981 & 2.4777113 & 0.0371585 \\
$\mathrm{C}$ & 0.0000170 & 1.7537023 & 0.0000171 \\
$\mathrm{C}$ & -1.2206346 & 2.4777558 & -0.0371620 \\
$\mathrm{C}$ & -0.0000097 & 0.3312616 & 0.0000514 \\
$\mathrm{C}$ & 1.2470902 & -0.3749364 & 0.0126377 \\
$\mathrm{C}$ & 2.4934432 & 0.3832004 & 0.0250636 \\
$\mathrm{C}$ & 1.2127577 & -1.7792831 & 0.0378100 \\
$\mathrm{H}$ & 2.1399773 & -2.3382659 & 0.0834987 \\
$\mathrm{C}$ & -1.2128550 & -1.7792413 & -0.0376107 \\
$\mathrm{C}$ & -1.2471346 & -0.3748927 & -0.0125093 \\
$\mathrm{C}$ & -2.4934581 & 0.3832912 & -0.0250084 \\
$\mathrm{H}$ & 2.0643161 & 4.4671203 & 0.0598649 \\
$\mathrm{H}$ & -2.0641779 & 4.4671950 & -0.0599710 \\
$\mathrm{H}$ & -2.1400942 & -2.3381946 & -0.0832852 \\
$\mathrm{C}$ & -3.8200151 & -0.2978255 & -0.0071802 \\
$\mathrm{C}$ & -4.1848630 & -1.1796098 & 1.0256603 \\
$\mathrm{C}$ & -4.7686446 & -0.0278142 & -1.0044875 \\
$\mathrm{C}$ & -5.4481345 & -1.7657883 & 1.0528297 \\
$\mathrm{H}$ & -3.4782016 & -1.3950435 & 1.8224173 \\
$\mathrm{C}$ & -6.0317688 & -0.6208434 & -0.9738837 \\
$\mathrm{H}$ & -4.5081882 & 0.6424510 & -1.8192366 \\
$\mathrm{C}$ & -6.3965799 & -1.4987590 & 0.0538091 \\
$\mathrm{H}$ & -5.7044331 & -2.4392973 & 1.8677344 \\
$\mathrm{H}$ & -6.7433615 & -0.3994265 & -1.7660206 \\
$\mathrm{C}$ & 3.8199799 & -0.2979539 & 0.0071877 \\
$\mathrm{C}$ & 4.1847070 & -1.1798472 & -1.0255584 \\
$\mathrm{C}$ & 4.7687051 & -0.0278949 & 1.0044368 \\
$\mathrm{C}$ & 5.4479721 & -1.7661135 & -1.0527398 \\
$\mathrm{H}$ & 3.4779568 & -1.3954171 & -1.8221997 \\
\hline & & & \\
\hline & & & \\
\hline & & &
\end{tabular}




\begin{tabular}{llll}
\hline $\mathrm{C}$ & 6.0317691 & -0.6209763 & 0.9738207 \\
$\mathrm{H}$ & 4.5082844 & 0.6423914 & 1.8191806 \\
$\mathrm{C}$ & 6.3965020 & -1.4989745 & -0.0538779 \\
$\mathrm{H}$ & 5.7041467 & -2.4397924 & -1.8675380 \\
$\mathrm{H}$ & 6.7433882 & -0.3995998 & 1.7659514 \\
$\mathrm{C}$ & -1.1463942 & 3.8807852 & -0.0343527 \\
$\mathrm{C}$ & 1.1465095 & 3.8807445 & 0.0342844 \\
$\mathrm{C}$ & 7.7697603 & -2.1268947 & -0.1011199 \\
$\mathrm{H}$ & 8.3924431 & -1.6614998 & -0.8763869 \\
$\mathrm{H}$ & 8.2935759 & -2.0149390 & 0.8533298 \\
$\mathrm{H}$ & 7.7133185 & -3.1961193 & -0.3350354 \\
$\mathrm{C}$ & -7.7695751 & -2.1272792 & 0.1007735 \\
$\mathrm{H}$ & -7.7119904 & -3.1987326 & 0.3241774 \\
$\mathrm{H}$ & -8.3890513 & -1.6695803 & 0.8831174 \\
$\mathrm{H}$ & -8.2972903 & -2.0064476 & -0.8504256 \\
$\mathrm{C}$ & -2.4544359 & 1.7528359 & -0.0534977 \\
$\mathrm{H}$ & -3.3843623 & 2.3155409 & -0.0551711 \\
$\mathrm{C}$ & 2.4544716 & 1.7527489 & 0.0535036 \\
$\mathrm{H}$ & 3.3844192 & 2.3154201 & 0.0551381 \\
$\mathrm{C}$ & -0.0000613 & -2.4633014 & 0.0001192 \\
$\mathrm{H}$ & -0.0000822 & -3.5497585 & 0.0001449 \\
$\mathrm{~N}$ & 0.0000708 & 4.5710213 & -0.0000481 \\
\hline & & & \\
\hline & & & \\
\hline & & &
\end{tabular}

$\mathrm{S}_{0}: \mathbf{5 j}$

$E=-1292.048011$ Hartree

\begin{tabular}{llll}
\hline Symbol & $\mathrm{X}$ & $\mathrm{Y}$ & $\mathrm{Z}$ \\
\hline $\mathrm{C}$ & -1.1465160 & -3.8656998 & 0.0310470 \\
$\mathrm{C}$ & -1.2205063 & -2.4627387 & 0.0338106 \\
$\mathrm{C}$ & -0.0000104 & -1.7384542 & -0.0000173 \\
$\mathrm{C}$ & 1.2204695 & -2.4627653 & -0.0338347 \\
$\mathrm{C}$ & 1.1464386 & -3.8657233 & -0.0310744 \\
$\mathrm{C}$ & -2.4544243 & -1.7379696 & 0.0472509 \\
$\mathrm{C}$ & 0.0000036 & -0.3160030 & -0.0000248 \\
$\mathrm{C}$ & -1.2468489 & 0.3903963 & 0.0110796 \\
$\mathrm{C}$ & -2.4922254 & -0.3686613 & 0.0206611 \\
$\mathrm{C}$ & -1.2127272 & 1.7947039 & 0.0364174 \\
$\mathrm{H}$ & -2.1393975 & 2.3546605 & 0.0822290 \\
$\mathrm{C}$ & 0.0000462 & 2.4787883 & -0.0000458 \\
C & 1.2128029 & 1.7946712 & -0.0364944 \\
$\mathrm{C}$ & 1.2468842 & 0.3903584 & -0.0111386 \\
\hline
\end{tabular}




\begin{tabular}{|c|c|c|c|}
\hline$C$ & 2.4922470 & -0.3687340 & -0.0207149 \\
\hline C & 2.4544120 & -1.7380368 & -0.0472839 \\
\hline $\mathrm{H}$ & 3.3841807 & -2.3009750 & -0.0457895 \\
\hline $\mathrm{H}$ & -3.3842103 & -2.3008797 & 0.0457845 \\
\hline $\mathrm{H}$ & -2.0643314 & -4.4520483 & 0.0539874 \\
\hline $\mathrm{H}$ & 2.0642381 & -4.4520970 & -0.0540084 \\
\hline $\mathrm{H}$ & 0.0000586 & 3.5651483 & -0.0000664 \\
\hline $\mathrm{H}$ & 2.1394916 & 2.3545939 & -0.0822977 \\
\hline $\mathrm{N}$ & -0.0000470 & -4.5558805 & -0.0000134 \\
\hline C & 3.8189916 & 0.3129579 & -0.0021123 \\
\hline C & 4.1818575 & 1.1870068 & 1.0378294 \\
\hline C & 4.7633024 & 0.0437466 & -1.0064742 \\
\hline C & 5.4441831 & 1.7776352 & 1.0763800 \\
\hline $\mathrm{H}$ & 3.4763255 & 1.3954325 & 1.8365025 \\
\hline C & 6.0302101 & 0.6273360 & -0.9841712 \\
\hline $\mathrm{H}$ & 4.4987599 & -0.6235711 & -1.8213278 \\
\hline C & 6.3470853 & 1.4868805 & 0.0600888 \\
\hline $\mathrm{H}$ & 5.7329165 & 2.4460780 & 1.8808006 \\
\hline $\mathrm{H}$ & 6.7608342 & 0.4271347 & -1.7609649 \\
\hline C & -3.8189669 & 0.3130360 & 0.0020904 \\
\hline C & -4.1818201 & 1.1872314 & -1.0377173 \\
\hline C & -4.7633159 & 0.0436300 & 1.0063859 \\
\hline C & -5.4441652 & 1.7778400 & -1.0762202 \\
\hline $\mathrm{H}$ & -3.4762782 & 1.3958209 & -1.8363377 \\
\hline C & -6.0302251 & 0.6271879 & 0.9841283 \\
\hline $\mathrm{H}$ & -4.4987523 & -0.6237854 & 1.8211521 \\
\hline C & -6.3470909 & 1.4868922 & -0.0600138 \\
\hline $\mathrm{H}$ & -5.7328699 & 2.4463872 & -1.8805626 \\
\hline $\mathrm{H}$ & -6.7608828 & 0.4268779 & 1.7608628 \\
\hline$F$ & -7.5724987 & 2.0588258 & -0.0898380 \\
\hline $\mathrm{F}$ & 7.5724651 & 2.0588752 & 0.0899647 \\
\hline
\end{tabular}

$\mathrm{S}_{0}: 5 \mathrm{~m}$

$E=-1322.548142$ Hartree

\begin{tabular}{llll}
\hline Symbol & $\mathrm{X}$ & $\mathrm{Y}$ & $\mathrm{Z}$ \\
\hline $\mathrm{C}$ & 1.1973078 & 4.0239413 & 0.1508238 \\
$\mathrm{C}$ & 1.2467697 & 2.6211706 & 0.0869500 \\
$\mathrm{C}$ & 0.0134719 & 1.9214908 & 0.0162576 \\
$\mathrm{C}$ & -1.1941761 & 2.6677880 & 0.0143865 \\
$\mathrm{C}$ & -1.0952301 & 4.0676936 & 0.0843697 \\
\hline
\end{tabular}




\begin{tabular}{|c|c|c|c|}
\hline $\mathrm{C}$ & 2.4674364 & 1.8748786 & 0.0660182 \\
\hline C & -0.0117778 & 0.5009099 & -0.0521325 \\
\hline C & 1.2230317 & -0.2267618 & -0.0732677 \\
\hline C & 2.4835722 & 0.5072974 & -0.0261851 \\
\hline C & 1.1638236 & -1.6298726 & -0.1149755 \\
\hline $\mathrm{H}$ & 2.0807686 & -2.2069620 & -0.0937899 \\
\hline C & -0.0606215 & -2.2897502 & -0.1886723 \\
\hline C & -1.2610933 & -1.5833865 & -0.1949936 \\
\hline C & -1.2715381 & -0.1816446 & -0.0990175 \\
\hline C & -2.5052653 & 0.5976597 & -0.0719661 \\
\hline C & -2.4403038 & 1.9664741 & -0.0336057 \\
\hline $\mathrm{H}$ & -3.3596203 & 2.5456549 & -0.0042946 \\
\hline $\mathrm{H}$ & 3.4067883 & 2.4211448 & 0.0902491 \\
\hline $\mathrm{H}$ & 2.1253943 & 4.5921356 & 0.2031003 \\
\hline $\mathrm{H}$ & -2.0026537 & 4.6705726 & 0.0877393 \\
\hline $\mathrm{H}$ & -0.0796291 & -3.3747465 & -0.2423451 \\
\hline $\mathrm{H}$ & -2.1974977 & -2.1231131 & -0.2721145 \\
\hline$N$ & 0.0631915 & 4.7351677 & 0.1505210 \\
\hline C & -3.8439201 & -0.0572197 & -0.0887277 \\
\hline C & -4.2250820 & -0.9973078 & 0.8795569 \\
\hline C & -4.7993767 & 0.3065331 & -1.0562475 \\
\hline C & -5.5021732 & -1.5626053 & 0.8920689 \\
\hline $\mathrm{H}$ & -3.5213352 & -1.2836179 & 1.6560230 \\
\hline C & -6.0725379 & -0.2485460 & -1.0594954 \\
\hline $\mathrm{H}$ & -4.5312431 & 1.0278009 & -1.8232731 \\
\hline C & -6.4352591 & -1.1898494 & -0.0839528 \\
\hline $\mathrm{H}$ & -5.7569355 & -2.2783401 & 1.6649337 \\
\hline $\mathrm{H}$ & -6.8026981 & 0.0298589 & -1.8133835 \\
\hline C & 3.7966146 & -0.1960605 & -0.0739798 \\
\hline C & 4.1453814 & -1.0503476 & -1.1385928 \\
\hline C & 4.7565572 & 0.0250159 & 0.9222012 \\
\hline C & 5.3937722 & -1.6551124 & -1.1976194 \\
\hline $\mathrm{H}$ & 3.4345628 & -1.2276837 & -1.9406977 \\
\hline C & 6.0160199 & -0.5785746 & 0.8794024 \\
\hline $\mathrm{H}$ & 4.5136832 & 0.6740145 & 1.7591712 \\
\hline C & 6.3405272 & -1.4265563 & -0.1861029 \\
\hline $\mathrm{H}$ & 5.6612145 & -2.3050295 & -2.0253054 \\
\hline $\mathrm{H}$ & 6.7242549 & -0.3847643 & 1.6766579 \\
\hline O & -7.7056584 & -1.6771970 & -0.1708549 \\
\hline C & -8.1282039 & -2.6381254 & 0.7927144 \\
\hline $\mathrm{H}$ & -8.0972931 & -2.2265613 & 1.8089257 \\
\hline
\end{tabular}




\begin{tabular}{llll}
\hline $\mathrm{H}$ & -9.1586038 & -2.8861092 & 0.5339534 \\
$\mathrm{H}$ & -7.5134550 & -3.5454136 & 0.7492114 \\
$\mathrm{O}$ & 7.5335600 & -2.0700946 & -0.3324620 \\
$\mathrm{C}$ & 8.5345625 & -1.8685785 & 0.6614694 \\
$\mathrm{H}$ & 9.3913267 & -2.4662648 & 0.3470351 \\
$\mathrm{H}$ & 8.8293553 & -0.8139959 & 0.7241883 \\
$\mathrm{H}$ & 8.1943264 & -2.2097469 & 1.6467967 \\
\hline
\end{tabular}

$S_{0}: 5 n$

$\mu=6.90 \mathrm{D}$

$E=-1361.356777$ Hartree

\begin{tabular}{llll}
\hline Symbol & $\mathrm{X}$ & $\mathrm{Y}$ & $\mathrm{Z}$ \\
\hline $\mathrm{C}$ & 1.1680880 & 4.1939991 & -0.0166979 \\
$\mathrm{C}$ & 1.2324494 & 2.7901190 & -0.0353124 \\
$\mathrm{C}$ & 0.0062843 & 2.0751730 & 0.0003951 \\
$\mathrm{C}$ & -1.2092758 & 2.8069796 & 0.0525686 \\
$\mathrm{C}$ & -1.1246171 & 4.2097756 & 0.0640838 \\
$\mathrm{C}$ & 2.4602927 & 2.0567779 & -0.0587613 \\
$\mathrm{C}$ & -0.0039979 & 0.6528323 & -0.0158977 \\
$\mathrm{C}$ & 1.2388225 & -0.0617808 & -0.0417641 \\
$\mathrm{C}$ & 2.4940453 & 0.6853012 & -0.0447639 \\
$\mathrm{C}$ & 1.1928291 & -1.4652621 & -0.0895770 \\
$\mathrm{H}$ & 2.1154514 & -2.0297099 & -0.1532565 \\
$\mathrm{C}$ & -0.0245238 & -2.1410258 & -0.0511504 \\
$\mathrm{C}$ & -1.2318321 & -1.4487218 & 0.0054535 \\
$\mathrm{C}$ & -1.2571459 & -0.0439806 & -0.0062645 \\
$\mathrm{C}$ & -2.5011274 & 0.7211006 & 0.0181595 \\
$\mathrm{C}$ & -2.4477005 & 2.0911452 & 0.0630319 \\
$\mathrm{H}$ & -3.3728949 & 2.6616994 & 0.0698448 \\
$\mathrm{H}$ & 3.3934862 & 2.6140692 & -0.0491599 \\
$\mathrm{H}$ & 2.0901269 & 4.7740437 & -0.0400868 \\
$\mathrm{H}$ & -2.0381474 & 4.8024571 & 0.1002771 \\
$\mathrm{H}$ & -0.0324792 & -3.2275164 & -0.0654201 \\
$\mathrm{H}$ & -2.1627016 & -2.0010573 & 0.0546203 \\
$\mathrm{~N}$ & 0.0267652 & 4.8920759 & 0.0308984 \\
$\mathrm{C}$ & -3.8305962 & 0.0526088 & 0.0012024 \\
$\mathrm{C}$ & -4.7981632 & 0.3569273 & 0.9723920 \\
$\mathrm{C}$ & -4.2063315 & -0.8542861 & -1.0047156 \\
$\mathrm{C}$ & -6.0673843 & -0.2111770 & 0.9540958 \\
$\mathrm{H}$ & -4.5439877 & 1.0431351 & 1.7763587 \\
\hline & & & \\
\hline & & &
\end{tabular}




\begin{tabular}{|c|c|c|c|}
\hline $\mathrm{C}$ & -5.4725241 & -1.4271749 & -1.0435546 \\
\hline $\mathrm{H}$ & -3.4989769 & -1.1073775 & -1.7900074 \\
\hline C & -6.4485025 & -1.1162148 & -0.0655539 \\
\hline $\mathrm{H}$ & -6.7624002 & 0.0490990 & 1.7432923 \\
\hline $\mathrm{H}$ & -5.7030717 & -2.1159358 & -1.8473062 \\
\hline C & 3.8132254 & -0.0030731 & -0.0360402 \\
\hline C & 4.7987864 & 0.3239473 & -0.9816123 \\
\hline C & 4.1670897 & -0.9425415 & 0.9478548 \\
\hline C & 6.0616440 & -0.2579211 & -0.9639559 \\
\hline $\mathrm{H}$ & 4.5671765 & 1.0470692 & -1.7596159 \\
\hline C & 5.4261927 & -1.5309212 & 0.9851278 \\
\hline $\mathrm{H}$ & 3.4514324 & -1.2017069 & 1.7235453 \\
\hline C & 6.4115593 & -1.2159982 & 0.0178359 \\
\hline $\mathrm{H}$ & 6.7763463 & 0.0332759 & -1.7242357 \\
\hline $\mathrm{H}$ & 5.6449658 & -2.2317709 & 1.7817355 \\
\hline C & -8.0032547 & -2.7382193 & -1.0512368 \\
\hline C & -8.6204803 & -1.4766435 & 1.0164021 \\
\hline $\mathrm{H}$ & -7.8415007 & -2.4102373 & -2.0847168 \\
\hline $\mathrm{H}$ & -7.3829464 & -3.6310989 & -0.8763178 \\
\hline $\mathrm{H}$ & -9.0515678 & -3.0256901 & -0.9569941 \\
\hline $\mathrm{H}$ & -8.8170486 & -0.4123428 & 1.1906890 \\
\hline $\mathrm{H}$ & -9.5745894 & -1.9562493 & 0.7926511 \\
\hline $\mathrm{H}$ & -8.2276243 & -1.9079031 & 1.9503907 \\
\hline$N$ & -7.7193393 & -1.6639144 & -0.1111349 \\
\hline C & 8.7069691 & -1.3134537 & -0.8420093 \\
\hline C & 8.0369899 & -2.6606972 & 1.1540680 \\
\hline $\mathrm{H}$ & 8.4192756 & -1.3902992 & -1.8972186 \\
\hline $\mathrm{H}$ & 8.9570477 & -0.2617635 & -0.6331736 \\
\hline $\mathrm{H}$ & 9.6069348 & -1.9138427 & -0.7009444 \\
\hline $\mathrm{H}$ & 7.3434522 & -3.5016500 & 1.2702071 \\
\hline $\mathrm{H}$ & 9.0296214 & -3.0746392 & 0.9700970 \\
\hline $\mathrm{H}$ & 8.0644730 & -2.1076842 & 2.1059702 \\
\hline$N$ & 7.6559005 & -1.8227241 & 0.0268012 \\
\hline
\end{tabular}

$\mathrm{S}_{0}$ (gas phase): $\mathbf{5 n}$

$E=-1361.346014$ Hartree

\begin{tabular}{llll}
\hline Symbol & $\mathrm{X}$ & $\mathrm{Y}$ & $\mathrm{Z}$ \\
\hline $\mathrm{C}$ & 1.1444501 & 4.2304140 & 0.0347291 \\
$\mathrm{C}$ & 1.2205183 & 2.8271203 & 0.0381592 \\
$\mathrm{C}$ & 0.0000363 & 2.1033380 & 0.0000194 \\
\hline
\end{tabular}




\begin{tabular}{|c|c|c|c|}
\hline$C$ & -1.2204052 & 2.8271913 & -0.0382136 \\
\hline C & -1.1442443 & 4.2304807 & -0.0349567 \\
\hline C & 2.4530392 & 2.1012537 & 0.0506156 \\
\hline C & -0.0000005 & 0.6809407 & 0.0000300 \\
\hline C & 1.2470444 & -0.0250485 & 0.0123803 \\
\hline C & 2.4951052 & 0.7313228 & 0.0213006 \\
\hline C & 1.2123985 & -1.4290627 & 0.0404818 \\
\hline $\mathrm{H}$ & 2.1410935 & -1.9852906 & 0.0924074 \\
\hline C & -0.0001432 & -2.1125231 & -0.0000796 \\
\hline C & -1.2126176 & -1.4289497 & -0.0405748 \\
\hline C & -1.2471064 & -0.0249387 & -0.0123481 \\
\hline C & -2.4951135 & 0.7315003 & -0.0211998 \\
\hline C & -2.4529834 & 2.1014160 & -0.0506368 \\
\hline $\mathrm{H}$ & -3.3837301 & 2.6633209 & -0.0443987 \\
\hline $\mathrm{H}$ & 3.3838222 & 2.6630967 & 0.0442470 \\
\hline $\mathrm{H}$ & 2.0615774 & 4.8188512 & 0.0598251 \\
\hline $\mathrm{H}$ & -2.0613335 & 4.8189730 & -0.0602018 \\
\hline $\mathrm{H}$ & -2.1413906 & -1.9850635 & -0.0924499 \\
\hline $\mathrm{N}$ & 0.0001231 & 4.9204423 & -0.0001445 \\
\hline C & -3.8187069 & 0.0506655 & 0.0003301 \\
\hline C & -4.1847533 & -0.8456836 & 1.0174593 \\
\hline C & -4.7859714 & 0.3287302 & -0.9768265 \\
\hline C & -5.4441152 & -1.4328401 & 1.0616487 \\
\hline $\mathrm{H}$ & -3.4735498 & -1.0786764 & 1.8054985 \\
\hline C & -6.0488183 & -0.2534287 & -0.9515995 \\
\hline $\mathrm{H}$ & -4.5359431 & 1.0054431 & -1.7899587 \\
\hline C & -6.4197736 & -1.1459982 & 0.0792842 \\
\hline $\mathrm{H}$ & -5.6682224 & -2.1128520 & 1.8748023 \\
\hline $\mathrm{H}$ & -6.7455038 & -0.0121568 & -1.7455161 \\
\hline C & 3.8186893 & 0.0504742 & -0.0002639 \\
\hline C & 4.1847202 & -0.8460213 & -1.0172927 \\
\hline C & 4.7859973 & 0.3287303 & 0.9768091 \\
\hline C & 5.4440392 & -1.4332443 & -1.0613994 \\
\hline $\mathrm{H}$ & 3.4735796 & -1.0789131 & -1.8054173 \\
\hline C & 6.0488175 & -0.2534915 & 0.9516983 \\
\hline $\mathrm{H}$ & 4.5360184 & 1.0056677 & 1.7897668 \\
\hline C & 6.4196258 & -1.1463983 & -0.0789446 \\
\hline $\mathrm{H}$ & 5.6682725 & -2.1132493 & -1.8745312 \\
\hline $\mathrm{H}$ & 6.7456390 & -0.0119896 & 1.7454281 \\
\hline $\mathrm{H}$ & -0.0001984 & -3.1995204 & -0.0001390 \\
\hline C & 8.5803721 & -1.5596900 & 1.0062261 \\
\hline
\end{tabular}




\begin{tabular}{llll}
\hline $\mathrm{H}$ & 9.5318018 & -2.0454765 & 0.7805761 \\
$\mathrm{H}$ & 8.7897066 & -0.5016852 & 1.2043377 \\
$\mathrm{H}$ & 8.1762732 & -2.0046849 & 1.9300910 \\
$\mathrm{C}$ & 7.9537979 & -2.7805849 & -1.0760666 \\
$\mathrm{H}$ & 7.7956733 & -2.4428495 & -2.1073271 \\
$\mathrm{H}$ & 8.9987905 & -3.0844851 & -0.9877990 \\
$\mathrm{H}$ & 7.3212451 & -3.6675697 & -0.9086262 \\
$\mathrm{C}$ & -7.9537418 & -2.7809402 & 1.0755857 \\
$\mathrm{H}$ & -7.7945417 & -2.4445287 & 2.1070677 \\
$\mathrm{H}$ & -8.9989776 & -3.0842283 & 0.9879656 \\
$\mathrm{H}$ & -7.3217210 & -3.6679993 & 0.9064233 \\
$\mathrm{C}$ & -8.5800727 & -1.5602314 & -1.0063598 \\
$\mathrm{H}$ & -9.5318959 & -2.0451214 & -0.7803908 \\
$\mathrm{H}$ & -8.7887366 & -0.5024476 & -1.2060801 \\
$\mathrm{H}$ & -8.1757320 & -2.0068430 & -1.9293654 \\
$\mathrm{~N}$ & -7.6894523 & -1.7088396 & 0.1316162 \\
$\mathrm{~N}$ & 7.6889690 & -1.7097084 & -0.1309295 \\
\hline
\end{tabular}

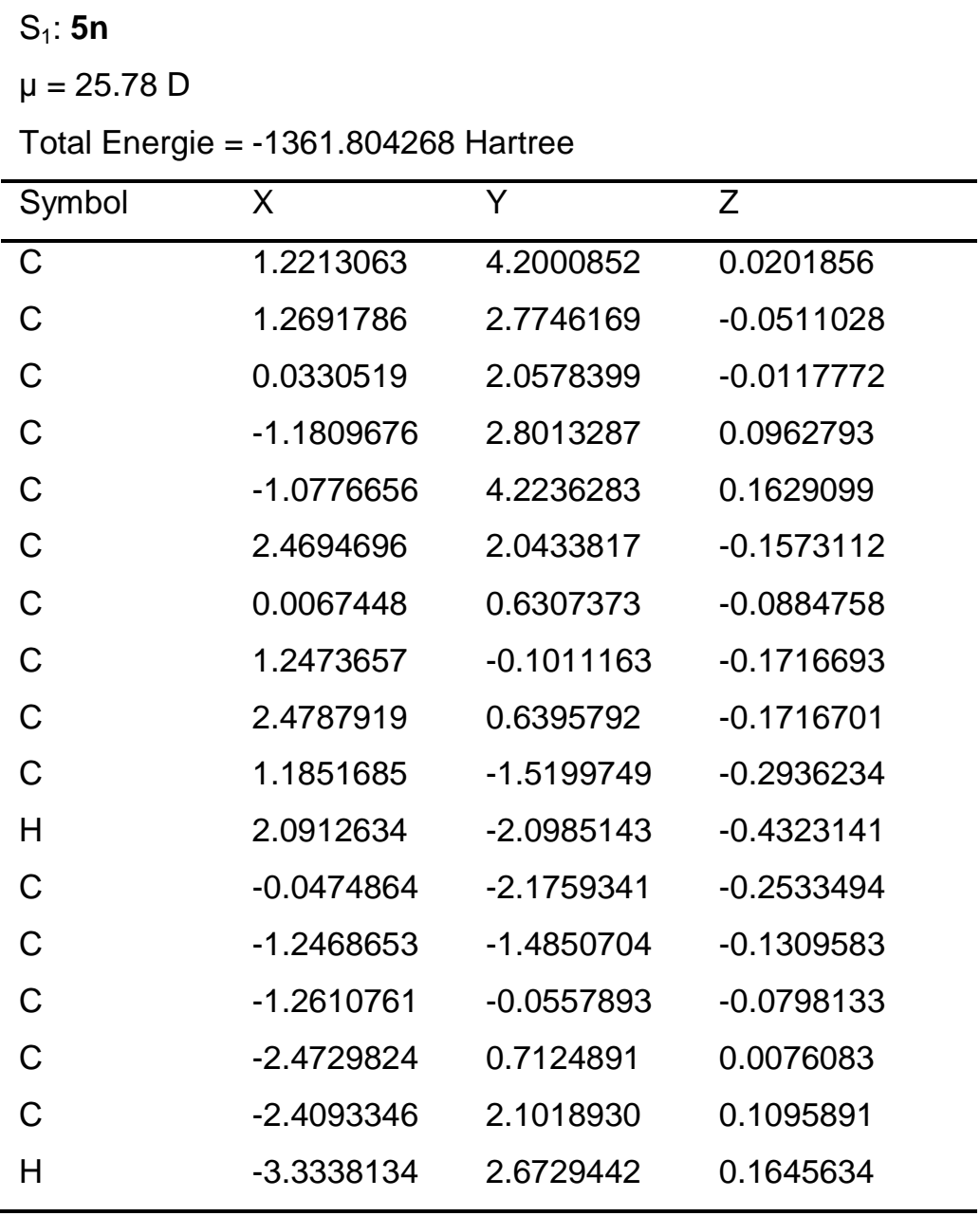




\begin{tabular}{|c|c|c|c|}
\hline$\overline{\mathrm{H}}$ & 3.4118384 & 2.5863059 & -0.1606314 \\
\hline $\mathrm{H}$ & 2.1471501 & 4.7727442 & -0.0117000 \\
\hline $\mathrm{H}$ & -1.9841297 & 4.8213324 & 0.2509270 \\
\hline $\mathrm{H}$ & -0.0675722 & -3.2625149 & -0.3205191 \\
\hline $\mathrm{H}$ & -2.1805467 & -2.0327587 & -0.0800033 \\
\hline $\mathrm{N}$ & 0.0817357 & 4.8925297 & 0.1219960 \\
\hline C & -3.8169751 & 0.0671489 & 0.0019590 \\
\hline C & -4.7415997 & 0.3081268 & 1.0321368 \\
\hline C & -4.2527307 & -0.7621242 & -1.0464295 \\
\hline C & -6.0244531 & -0.2407031 & 1.0264608 \\
\hline $\mathrm{H}$ & -4.4470121 & 0.9325100 & 1.8728912 \\
\hline C & -5.5310281 & -1.3169224 & -1.0731244 \\
\hline $\mathrm{H}$ & -3.5795332 & -0.9744374 & -1.8736436 \\
\hline C & -6.4644781 & -1.0622042 & -0.0384362 \\
\hline $\mathrm{H}$ & -6.6802925 & -0.0240031 & 1.8617037 \\
\hline $\mathrm{H}$ & -5.8008361 & -1.9447792 & -1.9144025 \\
\hline C & 3.8011583 & -0.0098254 & -0.0977138 \\
\hline C & 4.8733376 & 0.4214983 & -0.9234411 \\
\hline C & 4.0840784 & -1.0367450 & 0.8424544 \\
\hline C & 6.1298816 & -0.1400814 & -0.8436597 \\
\hline $\mathrm{H}$ & 4.6925089 & 1.2014969 & -1.6561361 \\
\hline C & 5.3338127 & -1.6061941 & 0.9467822 \\
\hline $\mathrm{H}$ & 3.2993974 & -1.3670659 & 1.5141242 \\
\hline C & 6.4025672 & -1.1815960 & 0.0966138 \\
\hline $\mathrm{H}$ & 6.9069023 & 0.2147464 & -1.5085629 \\
\hline $\mathrm{H}$ & 5.4995594 & -2.3741203 & 1.6913779 \\
\hline C & -8.0558084 & -2.6515089 & -1.0207398 \\
\hline C & -8.5698615 & -1.5306788 & 1.1317757 \\
\hline $\mathrm{H}$ & -7.8857373 & -2.3247015 & -2.0519318 \\
\hline $\mathrm{H}$ & -7.4505013 & -3.5561328 & -0.8414360 \\
\hline $\mathrm{H}$ & -9.1105972 & -2.9188738 & -0.9318307 \\
\hline $\mathrm{H}$ & -8.7186273 & -0.4969153 & 1.4609178 \\
\hline $\mathrm{H}$ & -9.5539936 & -1.9509458 & 0.9153645 \\
\hline $\mathrm{H}$ & -8.1246316 & -2.0976505 & 1.9668165 \\
\hline $\mathrm{N}$ & -7.7588436 & -1.5794918 & -0.0784380 \\
\hline C & 8.7302579 & -1.2943270 & -0.6825978 \\
\hline C & 7.9107549 & -2.8073253 & 1.1570006 \\
\hline $\mathrm{H}$ & 8.4749599 & -1.4506301 & -1.7356150 \\
\hline $\mathrm{H}$ & 8.9419922 & -0.2327706 & -0.5186860 \\
\hline $\mathrm{H}$ & 9.6248234 & -1.8687508 & -0.4518004 \\
\hline $\mathrm{H}$ & 7.2655955 & -3.6724259 & 0.9733349 \\
\hline
\end{tabular}




\begin{tabular}{llll}
\hline $\mathrm{H}$ & 8.9484833 & -3.1184712 & 1.0579601 \\
$\mathrm{H}$ & 7.7480182 & -2.4487475 & 2.1785362 \\
$\mathrm{~N}$ & 7.6376296 & -1.7433616 & 0.1858022 \\
\hline
\end{tabular}

$\mathrm{S}_{0}: 50$

$E=-1735.143229$ Hartree

\begin{tabular}{|c|c|c|c|}
\hline Symbol & $X$ & $\bar{Y}$ & $\bar{Z}$ \\
\hline $\mathrm{C}$ & 1.1469053 & 3.7009054 & 0.0020328 \\
\hline C & 1.2211699 & 2.2978613 & 0.0031451 \\
\hline C & -0.0000758 & 1.5740195 & -0.0000514 \\
\hline C & -1.2213137 & 2.2978809 & -0.0032160 \\
\hline C & -1.1470772 & 3.7009216 & -0.0020587 \\
\hline C & 2.4552462 & 1.5734445 & -0.0156379 \\
\hline C & -0.0000570 & 0.1514849 & -0.0001030 \\
\hline C & 1.2460031 & -0.5548855 & -0.0164409 \\
\hline C & 2.4914911 & 0.2035754 & -0.0365444 \\
\hline C & 1.2132700 & -1.9589606 & 0.0093404 \\
\hline $\mathrm{H}$ & 2.1412402 & -2.5184898 & 0.0344616 \\
\hline C & -0.0000849 & -2.6432838 & -0.0003815 \\
\hline C & -1.2134311 & -1.9589442 & -0.0099445 \\
\hline C & -1.2461044 & -0.5548720 & 0.0161507 \\
\hline C & -2.4915669 & 0.2036140 & 0.0364172 \\
\hline C & -2.4553892 & 1.5734609 & 0.0155883 \\
\hline $\mathrm{H}$ & -3.3856037 & 2.1341185 & 0.0463550 \\
\hline $\mathrm{H}$ & 3.3854602 & 2.1340775 & -0.0462752 \\
\hline $\mathrm{H}$ & 2.0649654 & 4.2873384 & 0.0018286 \\
\hline $\mathrm{H}$ & -2.0651336 & 4.2873663 & -0.0018416 \\
\hline $\mathrm{H}$ & -0.0000855 & -3.7296547 & -0.0005131 \\
\hline $\mathrm{H}$ & -2.1414270 & -2.5184597 & -0.0351061 \\
\hline$N$ & -0.0000873 & 4.3910556 & -0.0000043 \\
\hline C & 3.8078411 & -0.4823507 & -0.0833109 \\
\hline C & 4.2118057 & -1.4368967 & -1.0838236 \\
\hline C & 5.4958896 & -1.8724291 & -0.9257910 \\
\hline $\mathrm{H}$ & 3.5703516 & -1.7582954 & -1.8968314 \\
\hline $\mathrm{H}$ & 6.0472035 & -2.5706432 & -1.5411660 \\
\hline C & -3.8077725 & -0.4823315 & 0.0832665 \\
\hline C & -4.8208807 & -0.2291636 & -0.8103037 \\
\hline $\mathrm{H}$ & -4.7930158 & 0.4270032 & -1.6698415 \\
\hline C & -5.4953923 & -1.8725676 & 0.9265696 \\
\hline
\end{tabular}




\begin{tabular}{llll}
\hline $\mathrm{H}$ & -6.0466880 & -2.5707053 & 1.5420265 \\
$\mathrm{~S}$ & -6.2534135 & -1.1375054 & -0.4531309 \\
$\mathrm{C}$ & -4.2113095 & -1.4368762 & 1.0839820 \\
$\mathrm{H}$ & -3.5696252 & -1.7579261 & 1.8969944 \\
$\mathrm{~S}$ & 6.2534450 & -1.1373725 & 0.4533763 \\
$\mathrm{C}$ & 4.8207646 & -0.2290208 & 0.8104822 \\
$\mathrm{H}$ & 4.7928776 & 0.4274107 & 1.6698080 \\
\hline
\end{tabular}

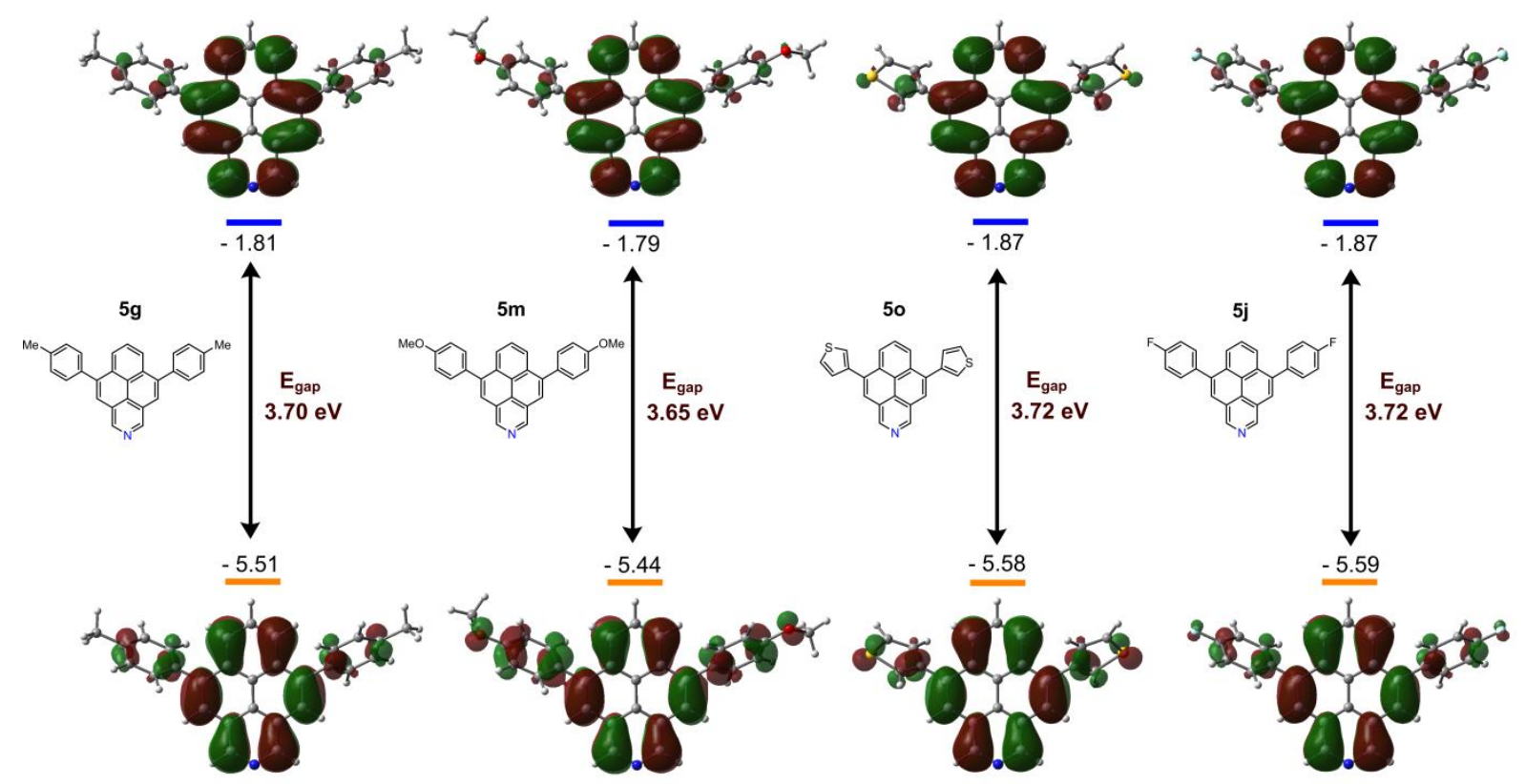

Figure S4. Calculated frontier molecular orbitals and energy levels at the B3LYP/6-31G(p) level of theory within IEFPCM of dichloromethane (isovalue $=0.02$ a.u.). 


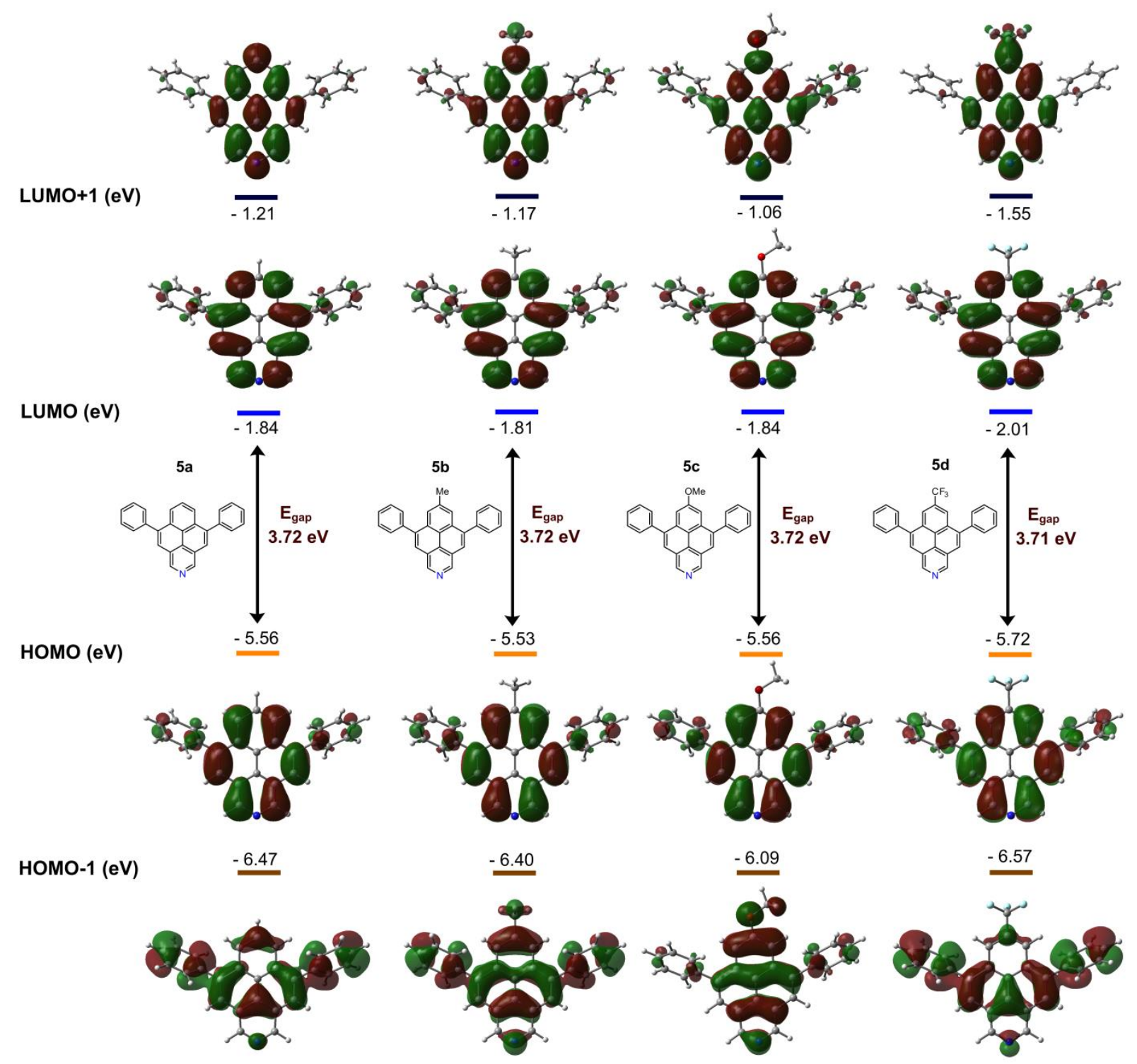

Figure S5. Calculated first four frontier molecular orbitals and energy levels at the B3LYP/6-31G(p) level of theory within IEFPCM of dichloromethane (isovalue $=0.02$ a.u.). 
Table S4: Calculated TD-DFT transitions of compound 2-azapyrene at B3LYP/6-31+G(d) level (IEFPCM).

\begin{tabular}{|c|c|c|c|c|c|}
\hline$\overline{S_{n}}$ & $E(e V)$ & $\lambda(\mathrm{nm})$ & $\bar{f}$ & Configuration & $\mathrm{Cl}$ coefficient \\
\hline \multirow[t]{2}{*}{$S_{1}$} & 3.6143 & 343.03 & 0.4103 & HOMO-1 $\rightarrow$ LUMO+1 & -0.17858 \\
\hline & & & & HOMO $\rightarrow$ LUMO & 0.68264 \\
\hline \multirow[t]{2}{*}{$\mathrm{S}_{2}$} & 3.6991 & 335.17 & 0.0446 & HOMO-1 $\rightarrow$ LUMO & 0.31362 \\
\hline & & & & HOMO $\rightarrow$ LUMO+1 & 0.63067 \\
\hline \multirow[t]{3}{*}{$\mathrm{S}_{3}$} & 4.3275 & 286.50 & 0.0074 & HOMO-3 $\rightarrow$ LUMO & -0.13588 \\
\hline & & & & HOMO-1 $\rightarrow$ LUMO & 0.15339 \\
\hline & & & & $\mathrm{HOMO} \rightarrow$ LUMO+2 & 0.67135 \\
\hline \multirow[t]{2}{*}{$\mathrm{S}_{4}$} & 4.3772 & 283.25 & 0.0000 & HOMO-2 $\rightarrow$ LUMO & 0.69770 \\
\hline & & & & HOMO-2 $\rightarrow$ LUMO+3 & -0.10771 \\
\hline \multirow[t]{4}{*}{$\mathrm{S}_{5}$} & 4.5621 & 271.77 & 0.1941 & HOMO-3 $\rightarrow$ LUMO & -0.34907 \\
\hline & & & & HOMO-1 $\rightarrow$ LUMO & 0.51136 \\
\hline & & & & HOMO $\rightarrow$ LUMO+1 & -0.23734 \\
\hline & & & & $\mathrm{HOMO} \rightarrow$ LUMO +2 & -0.21434 \\
\hline \multirow[t]{3}{*}{$\mathrm{S}_{6}$} & 4.6821 & 264.81 & 0.1307 & HOMO-3 $\rightarrow$ LUMO & 0.59476 \\
\hline & & & & HOMO-1 $\rightarrow$ LUMO & 0.31770 \\
\hline & & & & $\mathrm{HOMO} \rightarrow$ LUMO+2 & -0.17767 \\
\hline
\end{tabular}

Table S5: Calculated TD-DFT transitions of compound 2-azapyrene at CAM-B3LYP/6-31+G(d) level.

\begin{tabular}{|c|c|c|c|c|c|}
\hline$S_{n}$ & $E(e V)$ & $\lambda(\mathrm{nm})$ & $f$ & Configuration & $\mathrm{Cl}$ coefficient \\
\hline \multirow[t]{2}{*}{$\overline{S_{1}}$} & 3.9500 & 313.88 & 0.0250 & HOMO-1 $\rightarrow$ LUMO & 0.36321 \\
\hline & & & & HOMO $\rightarrow$ LUMO+1 & 0.59815 \\
\hline \multirow[t]{2}{*}{$S_{2}$} & 3.9989 & 310.04 & 0.3504 & HOMO-1 $\rightarrow$ LUMO+1 & -0.21813 \\
\hline & & & & HOMO $\rightarrow$ LUMO & 0.67118 \\
\hline \multirow[t]{2}{*}{$\mathrm{S}_{3}$} & 4.7296 & 262.14 & 0.0027 & HOMO-3 $\rightarrow$ LUMO & 0.11660 \\
\hline & & & & $\mathrm{HOMO} \rightarrow$ LUMO+2 & 0.68197 \\
\hline \multirow[t]{2}{*}{$\mathrm{S}_{4}$} & 4.8091 & 257.81 & 0.0000 & HOMO-2 $\rightarrow$ LUMO & 0.66012 \\
\hline & & & & HOMO-2 $\rightarrow$ LUMO+4 & 0.22663 \\
\hline \multirow[t]{3}{*}{$S_{5}$} & 5.0533 & 245.35 & 0.2202 & HOMO-3 $\rightarrow$ LUMO & -0.41202 \\
\hline & & & & HOMO-1 $\rightarrow$ LUMO & 0.48061 \\
\hline & & & & HOMO $\rightarrow$ LUMO+1 & -0.28122 \\
\hline \multirow[t]{2}{*}{$\mathrm{S}_{6}$} & 5.1397 & 241.23 & 0.0032 & HOMO-2 $\rightarrow$ LUMO+1 & 0.64845 \\
\hline & & & & HOMO-2 $\rightarrow$ LUMO+2 & -0.12862 \\
\hline
\end{tabular}




\begin{tabular}{ll}
\hline HOMO-2 $\rightarrow$ LUMO+13 & 0.16862 \\
HOMO-2 $\rightarrow$ LUMO+14 & 0.13283 \\
\hline
\end{tabular}

Table S6: Calculated TD-DFT transitions of compound 5a at B3LYP/6-31+G(d) level (IEFPCM).

\begin{tabular}{|c|c|c|c|c|c|}
\hline$S_{n}$ & $E(e V)$ & $\lambda(\mathrm{nm})$ & $f$ & Configuration & $\mathrm{Cl}$ coefficient \\
\hline \multirow[t]{2}{*}{$\mathrm{S}_{1}$} & 3.4587 & 358.03 & 0.3886 & HOMO-2 $\rightarrow$ LUMO+1 & 0.13990 \\
\hline & & & & HOMO $\rightarrow$ LUMO & 0.68597 \\
\hline \multirow[t]{3}{*}{$\mathrm{S}_{2}$} & 3.5918 & 345.19 & 0.0907 & HOMO-2 $\rightarrow$ LUMO & -0.22806 \\
\hline & & & & HOMO-1 $\rightarrow$ LUMO & 0.17130 \\
\hline & & & & $\mathrm{HOMO} \rightarrow$ LUMO+1 & 0.64258 \\
\hline \multirow[t]{3}{*}{$\mathrm{S}_{3}$} & 4.0141 & 308.87 & 0.2728 & HOMO-2 $\rightarrow$ LUMO & 0.13789 \\
\hline & & & & HOMO-1 $\rightarrow$ LUMO & 0.67577 \\
\hline & & & & $\mathrm{HOMO} \rightarrow \mathrm{LUMO}+2$ & -0.13316 \\
\hline \multirow[t]{2}{*}{$\mathrm{S}_{4}$} & 4.0200 & 308.42 & 0.0492 & HOMO-2 $\rightarrow$ LUMO & -0.13852 \\
\hline & & & & HOMO-2 $\rightarrow$ LUMO+3 & 0.68406 \\
\hline \multirow[t]{3}{*}{$S_{5}$} & 4.2860 & 289.28 & 0.0003 & HOMO-6 $\rightarrow$ LUMO & 0.47626 \\
\hline & & & & HOMO-5 $\rightarrow$ LUMO & -0.19556 \\
\hline & & & & HOMO-3 $\rightarrow$ LUMO & 0.46744 \\
\hline \multirow[t]{3}{*}{$\mathrm{S}_{6}$} & 4.4562 & 278.23 & 0.0008 & HOMO-6 $\rightarrow$ LUMO & -0.29964 \\
\hline & & & & HOMO-3 $\rightarrow$ LUMO & 0.26170 \\
\hline & & & & $\mathrm{HOMO} \rightarrow \mathrm{LUMO}+3$ & 0.56640 \\
\hline
\end{tabular}

Table S7: Calculated TD-DFT transitions of compound 5a at CAM-B3LYP/6-31+G(d) level.

\begin{tabular}{|c|c|c|c|c|c|}
\hline$S_{n}$ & $E(e V)$ & $\lambda(\mathrm{nm})$ & $f$ & Configuration & Cl coefficient \\
\hline \multirow[t]{3}{*}{$\mathrm{S}_{1}$} & 3.8751 & 319.95 & 0.0559 & HOMO-2 $\rightarrow$ LUMO & -0.25241 \\
\hline & & & & HOMO-1 $\rightarrow$ LUMO & 0.22264 \\
\hline & & & & HOMO $\rightarrow$ LUMO+1 & 0.60537 \\
\hline \multirow[t]{3}{*}{$\mathrm{S}_{2}$} & 3.8767 & 319.82 & 0.3454 & HOMO-2 $\rightarrow$ LUMO+1 & 0.15558 \\
\hline & & & & HOMO-1 $\rightarrow$ LUMO+1 & -0.13033 \\
\hline & & & & HOMO $\rightarrow$ LUMO & 0.67355 \\
\hline \multirow[t]{4}{*}{$\mathrm{S}_{3}$} & 4.4618 & 277.88 & 0.1807 & HOMO-1 $\rightarrow$ LUMO & 0.28434 \\
\hline & & & & HOMO $\rightarrow$ LUMO+1 & -0.12954 \\
\hline & & & & $\mathrm{HOMO} \rightarrow \mathrm{LUMO}+2$ & 0.58032 \\
\hline & & & & HOMO $\rightarrow$ LUMO+12 & -0.17711 \\
\hline
\end{tabular}




\begin{tabular}{|c|c|c|c|c|c|}
\hline \multirow[t]{4}{*}{$\mathrm{S}_{4}$} & 4.7777 & 259.51 & 0.0000 & HOMO-6 $\rightarrow$ LUMO & 0.58143 \\
\hline & & & & HOMO-6 $\rightarrow$ LUMO+3 & -0.18093 \\
\hline & & & & HOMO-5 $\rightarrow$ LUMO+11 & -0.13407 \\
\hline & & & & HOMO-3 $\rightarrow$ LUMO & 0.28989 \\
\hline \multirow[t]{4}{*}{$\mathrm{S}_{5}$} & 4.8177 & 257.35 & 0.1625 & HOMO-7 $\rightarrow$ LUMO & 0.18732 \\
\hline & & & & HOMO-2 $\rightarrow$ LUMO & 0.25495 \\
\hline & & & & HOMO-1 $\rightarrow$ LUMO & 0.52552 \\
\hline & & & & $\mathrm{HOMO} \rightarrow \mathrm{LUMO}+2$ & -0.27788 \\
\hline \multirow[t]{5}{*}{$S_{6}$} & 4.9775 & 249.09 & 0.5130 & HOMO-7 $\rightarrow$ LUMO & 0.13721 \\
\hline & & & & HOMO-2 $\rightarrow$ LUMO & 0.55379 \\
\hline & & & & HOMO-1 $\rightarrow$ LUMO & -0.21099 \\
\hline & & & & HOMO $\rightarrow$ LUMO+1 & 0.30534 \\
\hline & & & & $\mathrm{HOMO} \rightarrow$ LUMO+2 & 0.10012 \\
\hline
\end{tabular}

Table S8: Calculated TD-DFT transitions of compound $\mathbf{5 b}$ at B3LYP/6-31+G(d) level (IEFPCM).

\begin{tabular}{|c|c|c|c|c|c|}
\hline$S_{n}$ & $E(e V)$ & $\lambda(\mathrm{nm})$ & $f$ & Configuration & $\mathrm{Cl}$ coefficient \\
\hline \multirow[t]{3}{*}{$\mathrm{S}_{1}$} & 3.4412 & 360.29 & 0.3760 & HOMO-2 $\rightarrow$ LUMO+1 & 0.11911 \\
\hline & & & & HOMO-1 $\rightarrow$ LUMO+1 & -0.11796 \\
\hline & & & & HOMO $\rightarrow$ LUMO & 0.68391 \\
\hline \multirow[t]{3}{*}{$\mathrm{S}_{2}$} & 3.5866 & 345.69 & 0.0537 & HOMO-2 $\rightarrow$ LUMO & -0.19966 \\
\hline & & & & HOMO-1 $\rightarrow$ LUMO & 0.27921 \\
\hline & & & & HOMO $\rightarrow$ LUMO+1 & 0.61332 \\
\hline \multirow[t]{3}{*}{$\mathrm{S}_{3}$} & 3.9807 & 311.47 & 0.2601 & HOMO-1 $\rightarrow$ LUMO & 0.35149 \\
\hline & & & & HOMO $\rightarrow$ LUMO+1 & -0.16748 \\
\hline & & & & $\mathrm{HOMO} \rightarrow$ LUMO+2 & 0.58025 \\
\hline \multirow[t]{4}{*}{$\mathrm{S}_{4}$} & 4.0108 & 309.12 & 0.1340 & HOMO-6 $\rightarrow$ LUMO & 0.35573 \\
\hline & & & & HOMO-6 $\rightarrow$ LUMO+3 & 0.49588 \\
\hline & & & & HOMO-5 $\rightarrow$ LUMO+11 & -0.11220 \\
\hline & & & & HOMO-3 $\rightarrow$ LUMO & -0.31950 \\
\hline \multirow[t]{5}{*}{$S_{5}$} & 4.3214 & 286.91 & 0.2858 & HOMO-6 $\rightarrow$ LUMO & 0.16558 \\
\hline & & & & HOMO-2 $\rightarrow$ LUMO & 0.53206 \\
\hline & & & & HOMO-1 $\rightarrow$ LUMO & -0.20556 \\
\hline & & & & HOMO $\rightarrow$ LUMO+1 & 0.26200 \\
\hline & & & & $\mathrm{HOMO} \rightarrow \mathrm{LUMO}+2$ & 0.21216 \\
\hline
\end{tabular}




\begin{tabular}{llllll}
\hline $\mathrm{S}_{6}$ & 4.3531 & 284.82 & 0.0013 & HOMO-6 $\rightarrow$ LUMO & 0.67379 \\
& & & & HOMO-2 $\rightarrow$ LUMO & -0.16782 \\
\hline
\end{tabular}

Table S9: Calculated TD-DFT transitions of compound $\mathbf{5 b}$ at CAM-B3LYP/6-31+G(d) level.

\begin{tabular}{|c|c|c|c|c|c|}
\hline$\overline{S_{n}}$ & $E(e V)$ & $\lambda(\mathrm{nm})$ & $f$ & Configuration & $\mathrm{Cl}$ coefficient \\
\hline \multirow[t]{3}{*}{$\mathrm{S}_{1}$} & 3.8596 & 321.23 & 0.3327 & HOMO-2 $\rightarrow$ LUMO+1 & 0.10800 \\
\hline & & & & HOMO-1 $\rightarrow$ LUMO+1 & 0.17961 \\
\hline & & & & HOMO $\rightarrow$ LUMO & 0.67078 \\
\hline \multirow[t]{3}{*}{$\mathrm{S}_{2}$} & 3.8684 & 320.51 & 0.0318 & HOMO-2 $\rightarrow$ LUMO & -0.18284 \\
\hline & & & & HOMO-1 $\rightarrow$ LUMO & -0.33242 \\
\hline & & & & HOMO $\rightarrow$ LUMO+1 & 0.57466 \\
\hline \multirow[t]{6}{*}{$\mathrm{S}_{3}$} & 4.4380 & 279.37 & 0.2367 & HOMO-2 $\rightarrow$ LUMO & 0.11837 \\
\hline & & & & HOMO-1 $\rightarrow$ LUMO & -0.28358 \\
\hline & & & & HOMO $\rightarrow$ LUMO+1 & -0.19113 \\
\hline & & & & HOMO $\rightarrow$ LUMO+2 & 0.55453 \\
\hline & & & & $\mathrm{HOMO} \rightarrow \mathrm{LUMO}+8$ & 0.13398 \\
\hline & & & & HOMO $\rightarrow$ LUMO+9 & -0.10121 \\
\hline \multirow[t]{6}{*}{$\mathrm{S}_{4}$} & 4.7435 & 261.38 & 0.0445 & HOMO-7 $\rightarrow$ LUMO & 0.14177 \\
\hline & & & & HOMO-6 $\rightarrow$ LUMO & 0.46748 \\
\hline & & & & HOMO-6 $\rightarrow$ LUMO+11 & 0.12251 \\
\hline & & & & HOMO-2 $\rightarrow$ LUMO & -0.31045 \\
\hline & & & & HOMO-1 $\rightarrow$ LUMO & 0.25675 \\
\hline & & & & $\mathrm{HOMO} \rightarrow$ LUMO+2 & 0.20994 \\
\hline \multirow[t]{6}{*}{$\mathrm{S}_{5}$} & 4.8561 & 255.32 & 0.0950 & HOMO-7 $\rightarrow$ LUMO & -0.11527 \\
\hline & & & & HOMO-6 $\rightarrow$ LUMO & 0.46997 \\
\hline & & & & HOMO-6 $\rightarrow$ LUMO+11 & 0.10192 \\
\hline & & & & HOMO-2 $\rightarrow$ LUMO & 0.30421 \\
\hline & & & & HOMO-1 $\rightarrow$ LUMO & -0.28729 \\
\hline & & & & HOMO $\rightarrow$ LUMO+2 & -0.19921 \\
\hline \multirow[t]{5}{*}{$\mathrm{S}_{6}$} & 4.8650 & 254.85 & 0.4637 & HOMO-6 $\rightarrow$ LUMO & -0.12436 \\
\hline & & & & HOMO-2 $\rightarrow$ LUMO & 0.43976 \\
\hline & & & & HOMO-1 $\rightarrow$ LUMO & 0.35947 \\
\hline & & & & $\mathrm{HOMO} \rightarrow \mathrm{LUMO}+1$ & 0.33231 \\
\hline & & & & HOMO $\rightarrow$ LUMO+2 & 0.17066 \\
\hline
\end{tabular}


Table S10: Calculated TD-DFT transitions of compound $\mathbf{5 c}$ at B3LYP/6-31+G(d) level (IEFPCM).

\begin{tabular}{|c|c|c|c|c|c|}
\hline$S_{n}$ & $E(e V)$ & $\lambda(\mathrm{nm})$ & $f$ & Configuration & $\mathrm{Cl}$ coefficient \\
\hline \multirow[t]{2}{*}{$\mathrm{S}_{1}$} & 3.4335 & 361.10 & 0.3272 & HOMO-1 $\rightarrow$ LUMO+1 & -0.18245 \\
\hline & & & & HOMO $\rightarrow$ LUMO & 0.67512 \\
\hline \multirow[t]{2}{*}{$\mathrm{S}_{2}$} & 3.5393 & 350.30 & 0.0107 & HOMO-1 $\rightarrow$ LUMO & 0.56058 \\
\hline & & & & HOMO $\rightarrow$ LUMO+1 & 0.41207 \\
\hline \multirow[t]{4}{*}{$\mathrm{S}_{3}$} & 3.9467 & 314.15 & 0.5012 & HOMO-2 $\rightarrow$ LUMO & -0.11566 \\
\hline & & & & HOMO-1 $\rightarrow$ LUMO & -0.32229 \\
\hline & & & & $\mathrm{HOMO} \rightarrow \mathrm{LUMO}+1$ & 0.48737 \\
\hline & & & & $\mathrm{HOMO} \rightarrow$ LUMO+2 & -0.36119 \\
\hline \multirow[t]{2}{*}{$\mathrm{S}_{4}$} & 4.0138 & 308.90 & 0.0424 & HOMO-2 $\rightarrow$ LUMO & 0.61690 \\
\hline & & & & $\mathrm{HOMO} \rightarrow$ LUMO+2 & -0.30852 \\
\hline \multirow[t]{4}{*}{$\mathrm{S}_{5}$} & 4.1749 & 296.98 & 0.0961 & HOMO-2 $\rightarrow$ LUMO & 0.29408 \\
\hline & & & & HOMO-1 $\rightarrow$ LUMO & -0.24199 \\
\hline & & & & HOMO $\rightarrow$ LUMO+1 & 0.27543 \\
\hline & & & & $\mathrm{HOMO} \rightarrow \mathrm{LUMO}+2$ & 0.50553 \\
\hline $\mathrm{S}_{6}$ & 4.3224 & 286.84 & 0.0048 & HOMO-6 $\rightarrow$ LUMO & 0.69125 \\
\hline
\end{tabular}

Table S11: Calculated TD-DFT transitions of compound 5c at CAM-B3LYP/6-31+G(d) level.

\begin{tabular}{|c|c|c|c|c|c|}
\hline$S_{n}$ & $E(e V)$ & $\lambda(\mathrm{nm})$ & $f$ & Configuration & $\mathrm{Cl}$ coefficient \\
\hline \multirow[t]{5}{*}{$\mathrm{S}_{1}$} & 3.8409 & 322.80 & 0.1009 & HOMO-1 $\rightarrow$ LUMO & 0.41691 \\
\hline & & & & HOMO-1 $\rightarrow$ LUMO+1 & 0.13918 \\
\hline & & & & HOMO $\rightarrow$ LUMO & 0.39337 \\
\hline & & & & $\mathrm{HOMO} \rightarrow \mathrm{LUMO}+1$ & -0.33948 \\
\hline & & & & HOMO $\rightarrow$ LUMO+2 & -0.11237 \\
\hline \multirow[t]{4}{*}{$\mathrm{S}_{2}$} & 3.8643 & 320.58 & 0.1969 & HOMO-1 $\rightarrow$ LUMO & -0.28456 \\
\hline & & & & HOMO-1 $\rightarrow$ LUMO+1 & 0.16798 \\
\hline & & & & HOMO $\rightarrow$ LUMO & 0.53426 \\
\hline & & & & HOMO $\rightarrow$ LUMO+1 & 0.28801 \\
\hline \multirow[t]{5}{*}{$\mathrm{S}_{3}$} & 4.4432 & 279.04 & 0.3819 & HOMO-2 $\rightarrow$ LUMO & -0.18184 \\
\hline & & & & HOMO-1 $\rightarrow$ LUMO & -0.25635 \\
\hline & & & & HOMO $\rightarrow$ LUMO+1 & -0.38932 \\
\hline & & & & HOMO $\rightarrow$ LUMO+2 & 0.43675 \\
\hline & & & & $\mathrm{HOMO} \rightarrow$ LUMO+9 & 0.13267 \\
\hline
\end{tabular}




\begin{tabular}{|c|c|c|c|c|c|}
\hline \multirow[t]{6}{*}{$\overline{S_{4}}$} & 4.7023 & 263.66 & 0.0993 & HOMO-6 $\rightarrow$ LUMO & 0.19918 \\
\hline & & & & HOMO-2 $\rightarrow$ LUMO & 0.22833 \\
\hline & & & & HOMO-1 $\rightarrow$ LUMO & 0.48675 \\
\hline & & & & HOMO $\rightarrow$ LUMO & -0.22070 \\
\hline & & & & HOMO $\rightarrow$ LUMO+1 & -0.27239 \\
\hline & & & & $\mathrm{HOMO} \rightarrow$ LUMO+2 & -0.12650 \\
\hline \multirow[t]{4}{*}{$\mathrm{S}_{5}$} & 4.7453 & 261.28 & 0.1301 & HOMO-6 $\rightarrow$ LUMO & 0.51468 \\
\hline & & & & HOMO-1 $\rightarrow$ LUMO & 0.24148 \\
\hline & & & & HOMO $\rightarrow$ LUMO+1 & 0.18452 \\
\hline & & & & $\mathrm{HOMO} \rightarrow$ LUMO+2 & 0.30166 \\
\hline \multirow[t]{6}{*}{$\mathrm{S}_{6}$} & 4.8586 & 255.19 & 0.1829 & HOMO-7 $\rightarrow$ LUMO & 0.10769 \\
\hline & & & & HOMO-6 $\rightarrow$ LUMO & -0.35042 \\
\hline & & & & HOMO-2 $\rightarrow$ LUMO & 0.37835 \\
\hline & & & & HOMO-1 $\rightarrow$ LUMO & 0.22698 \\
\hline & & & & $\mathrm{HOMO} \rightarrow \mathrm{LUMO}+1$ & 0.11544 \\
\hline & & & & $\mathrm{HOMO} \rightarrow \mathrm{LUMO}+2$ & 0.32875 \\
\hline
\end{tabular}

Table S12: Calculated TD-DFT transitions of compound 5d at B3LYP/6-31+G(d) level (IEFPCM).

\begin{tabular}{cccclr}
\hline $\mathrm{S}_{\mathrm{n}}$ & $\mathrm{E}(\mathrm{eV})$ & $\lambda(\mathrm{nm})$ & $f$ & Configuration & Cl coefficient \\
\hline $\mathrm{S}_{1}$ & 3.4398 & 360.44 & 0.3795 & HOMO-5 $\rightarrow$ LUMO+1 & 0.14092 \\
& & & & HOMO $\rightarrow$ LUMO & 0.68531 \\
$\mathrm{~S}_{2}$ & 3.4634 & 357.98 & 0.1410 & HOMO-5 $\rightarrow$ LUMO & -0.20066 \\
& & & & HOMO $\rightarrow$ LUMO+1 & 0.66753 \\
$\mathrm{~S}_{3}$ & 3.9048 & 317.52 & 0.1701 & HOMO-1 $\rightarrow$ LUMO & 0.68855 \\
$\mathrm{~S}_{4}$ & 4.0575 & 305.57 & 0.0901 & HOMO $\rightarrow$ LUMO+2 & 0.69302 \\
$\mathrm{~S}_{5}$ & 4.2746 & 290.05 & 0.0113 & HOMO-6 $\rightarrow$ LUMO & 0.69181 \\
$\mathrm{~S}_{6}$ & 4.3265 & 286.57 & 0.0018 & HOMO-4 $\rightarrow$ LUMO & -0.14359 \\
& & & & HOMO-2 $\rightarrow$ LUMO & 0.53255 \\
& & & & HOMO-1 $\rightarrow$ LUMO+1 & -0.41635 \\
\hline
\end{tabular}

Table S13: Calculated TD-DFT transitions of compound 5d at CAM-B3LYP/6-31+G(d) level.

\begin{tabular}{cccclr}
\hline $\mathrm{S}_{\mathrm{n}}$ & $\mathrm{E}(\mathrm{eV})$ & $\lambda(\mathrm{nm})$ & $f$ & Configuration & Cl coefficient \\
\hline $\mathrm{S}_{1}$ & 3.7831 & 327.73 & 0.0942 & HOMO-3 $\rightarrow$ LUMO & 0.24449 \\
& & & & HOMO-1 $\rightarrow$ LUMO & 0.13251 \\
\hline
\end{tabular}




\begin{tabular}{|c|c|c|c|c|c|}
\hline & & & & $\mathrm{HOMO} \rightarrow \mathrm{LUMO}+1$ & 0.63208 \\
\hline \multirow[t]{2}{*}{$\mathrm{S}_{2}$} & 3.8680 & 320.54 & 0.3416 & HOMO-3 $\rightarrow$ LUMO+1 & -0.17093 \\
\hline & & & & HOMO $\rightarrow$ LUMO & 0.67281 \\
\hline \multirow[t]{4}{*}{$\mathrm{S}_{3}$} & 4.4705 & 277.34 & 0.2142 & HOMO-7 $\rightarrow$ LUMO & -0.11692 \\
\hline & & & & HOMO-1 $\rightarrow$ LUMO & 0.36954 \\
\hline & & & & HOMO $\rightarrow$ LUMO+2 & 0.54467 \\
\hline & & & & $\mathrm{HOMO} \rightarrow \mathrm{LUMO}+8$ & -0.14417 \\
\hline \multirow[t]{5}{*}{$\mathrm{S}_{4}$} & 4.6933 & 264.17 & 0.0371 & HOMO-7 $\rightarrow$ LUMO & 0.13870 \\
\hline & & & & HOMO-6 $\rightarrow$ LUMO & 0.40592 \\
\hline & & & & HOMO-6 $\rightarrow$ LUMO+10 & 0.13290 \\
\hline & & & & HOMO-1 $\rightarrow$ LUMO & 0.39784 \\
\hline & & & & $\mathrm{HOMO} \rightarrow \mathrm{LUMO}+2$ & 0.30889 \\
\hline \multirow[t]{4}{*}{$\mathrm{S}_{5}$} & 4.7990 & 258.35 & 0.0771 & HOMO-6 $\rightarrow$ LUMO & 0.52020 \\
\hline & & & & HOMO-6 $\rightarrow$ LUMO+10 & -0.14314 \\
\hline & & & & HOMO-1 $\rightarrow$ LUMO & 0.35160 \\
\hline & & & & $\mathrm{HOMO} \rightarrow$ LUMO+2 & -0.20343 \\
\hline \multirow[t]{5}{*}{$\mathrm{S}_{6}$} & 5.0183 & 247.06 & 0.0051 & HOMO-7 $\rightarrow$ LUMO+1 & -0.12884 \\
\hline & & & & HOMO-6 $\rightarrow$ LUMO+1 & -0.56039 \\
\hline & & & & HOMO-6 $\rightarrow$ LUMO+8 & -0.10605 \\
\hline & & & & HOMO-6 $\rightarrow$ LUMO+19 & 0.17621 \\
\hline & & & & HOMO-1 $\rightarrow$ LUMO+1 & 0.25824 \\
\hline
\end{tabular}

Table S14: Calculated TD-DFT transitions of compound $\mathbf{5 g}$ at B3LYP/6-31+G(d) level (IEFPCM).

\begin{tabular}{|c|c|c|c|c|c|}
\hline$\overline{S_{n}}$ & $E(e V)$ & $\lambda(\mathrm{nm})$ & $f$ & Configuration & $\mathrm{Cl}$ coefficient \\
\hline \multirow[t]{2}{*}{$\mathrm{S}_{1}$} & 3.4350 & 360.94 & 0.3857 & HOMO-3 $\rightarrow$ LUMO+1 & 0.14174 \\
\hline & & & & HOMO $\rightarrow$ LUMO & 0.68590 \\
\hline \multirow[t]{3}{*}{$\mathrm{S}_{2}$} & 3.5747 & 346.84 & 0.0850 & HOMO-3 $\rightarrow$ LUMO & -0.23126 \\
\hline & & & & HOMO-3 $\rightarrow$ LUMO & -0.17916 \\
\hline & & & & HOMO $\rightarrow$ LUMO+1 & 0.63904 \\
\hline \multirow[t]{2}{*}{$\mathrm{S}_{3}$} & 3.8552 & 321.60 & 0.2968 & HOMO-1 $\rightarrow$ LUMO & 0.67835 \\
\hline & & & & HOMO $\rightarrow$ LUMO+1 & 0.15835 \\
\hline \multirow[t]{2}{*}{$\mathrm{S}_{4}$} & 4.0367 & 307.14 & 0.0660 & HOMO-3 $\rightarrow$ LUMO & 0.14070 \\
\hline & & & & $\mathrm{HOMO} \rightarrow \mathrm{LUMO}+2$ & 0.68280 \\
\hline $\mathrm{S}_{5}$ & 4.1950 & 295.55 & 0.0025 & HOMO-6 $\rightarrow$ LUMO & -0.25118 \\
\hline
\end{tabular}




\begin{tabular}{|c|c|c|c|c|c|}
\hline & & & & HOMO-2 $\rightarrow$ LUMO & 0.64204 \\
\hline \multirow[t]{5}{*}{$\mathrm{S}_{6}$} & 4.3972 & 281.96 & 0.0001 & HOMO-6 $\rightarrow$ LUMO & 0.61509 \\
\hline & & & & HOMO-5 $\rightarrow$ LUMO & -0.15974 \\
\hline & & & & HOMO-2 $\rightarrow$ LUMO & 0.21886 \\
\hline & & & & HOMO-1 $\rightarrow$ LUMO+1 & 0.12544 \\
\hline & & & & $\mathrm{HOMO} \rightarrow \mathrm{LUMO}+3$ & -0.13966 \\
\hline
\end{tabular}

Table S15: Calculated TD-DFT transitions of compound 5g at CAM-B3LYP/6-31+G(d) level.

\begin{tabular}{|c|c|c|c|c|c|}
\hline$S_{n}$ & $E(e V)$ & $\lambda(\mathrm{nm})$ & $f$ & Configuration & $\mathrm{Cl}$ coefficient \\
\hline \multirow[t]{3}{*}{$\mathrm{S}_{1}$} & 3.8667 & 320.65 & 0.3514 & HOMO-3 $\rightarrow$ LUMO+1 & -0.17498 \\
\hline & & & & HOMO-1 $\rightarrow$ LUMO+1 & 0.10010 \\
\hline & & & & HOMO $\rightarrow$ LUMO & 0.67349 \\
\hline \multirow[t]{3}{*}{$\mathrm{S}_{2}$} & 3.8681 & 320.53 & 0.0607 & HOMO-3 $\rightarrow$ LUMO & 0.28613 \\
\hline & & & & HOMO-1 $\rightarrow$ LUMO & -0.17277 \\
\hline & & & & $\mathrm{HOMO} \rightarrow$ LUMO+1 & 0.60513 \\
\hline \multirow[t]{5}{*}{$\mathrm{S}_{3}$} & 4.4464 & 278.84 & 0.2697 & HOMO-7 $\rightarrow$ LUMO & -0.11516 \\
\hline & & & & HOMO-1 $\rightarrow$ LUMO & 0.35139 \\
\hline & & & & $\mathrm{HOMO} \rightarrow \mathrm{LUMO}+1$ & 0.13818 \\
\hline & & & & $\mathrm{HOMO} \rightarrow \mathrm{LUMO}+2$ & 0.54023 \\
\hline & & & & $\mathrm{HOMO} \rightarrow \mathrm{LUMO}+8$ & -0.15559 \\
\hline \multirow[t]{6}{*}{$\mathrm{S}_{4}$} & 4.7554 & 260.73 & 0.1448 & HOMO-7 $\rightarrow$ LUMO & -0.17972 \\
\hline & & & & HOMO-3 $\rightarrow$ LUMO & 0.14904 \\
\hline & & & & HOMO-2 $\rightarrow$ LUMO+2 & -0.10308 \\
\hline & & & & HOMO-1 $\rightarrow$ LUMO & 0.52183 \\
\hline & & & & $\mathrm{HOMO} \rightarrow \mathrm{LUMO}+2$ & -0.34272 \\
\hline & & & & $\mathrm{HOMO} \rightarrow \mathrm{LUMO}+8$ & 0.10910 \\
\hline \multirow[t]{3}{*}{$\mathrm{S}_{5}$} & 4.7845 & 259.14 & 0.0001 & HOMO-6 $\rightarrow$ LUMO & 0.61103 \\
\hline & & & & HOMO-6 $\rightarrow$ LUMO+10 & -0.17971 \\
\hline & & & & HOMO-2 $\rightarrow$ LUMO & -0.24990 \\
\hline \multirow[t]{4}{*}{$\mathrm{S}_{6}$} & 4.9686 & 249.54 & 0.5293 & HOMO-7 $\rightarrow$ LUMO & -0.16592 \\
\hline & & & & HOMO-3 $\rightarrow$ LUMO & 0.57312 \\
\hline & & & & HOMO-1 $\rightarrow$ LUMO & -0.10324 \\
\hline & & & & HOMO $\rightarrow$ LUMO+1 & -0.29903 \\
\hline
\end{tabular}


Table S16: Calculated TD-DFT transitions of compound $\mathbf{5 j}$ at B3LYP/6-31+G(d) level (IEFPCM).

\begin{tabular}{|c|c|c|c|c|c|}
\hline$S_{n}$ & $E(e V)$ & $\lambda(\mathrm{nm})$ & $f$ & Configuration & $\mathrm{Cl}$ coefficient \\
\hline \multirow[t]{2}{*}{$\mathrm{S}_{1}$} & 3.4594 & 358.40 & 0.3855 & HOMO-3 $\rightarrow$ LUMO+1 & -0.14172 \\
\hline & & & & HOMO $\rightarrow$ LUMO & 0.68577 \\
\hline \multirow[t]{3}{*}{$\mathrm{S}_{2}$} & 3.5943 & 344.95 & 0.0842 & HOMO-3 $\rightarrow$ LUMO & 0.23486 \\
\hline & & & & HOMO-1 $\rightarrow$ LUMO & 0.17238 \\
\hline & & & & HOMO $\rightarrow$ LUMO+1 & 0.63972 \\
\hline \multirow[t]{3}{*}{$\mathrm{S}_{3}$} & 3.9762 & 311.81 & 0.2476 & HOMO-3 $\rightarrow$ LUMO & -0.12958 \\
\hline & & & & HOMO-1 $\rightarrow$ LUMO & 0.67565 \\
\hline & & & & HOMO $\rightarrow$ LUMO+1 & -0.13805 \\
\hline \multirow[t]{2}{*}{$\mathrm{S}_{4}$} & 4.0263 & 307.94 & 0.0584 & HOMO-3 $\rightarrow$ LUMO & 0.11523 \\
\hline & & & & $\mathrm{HOMO} \rightarrow \mathrm{LUMO}+2$ & 0.68538 \\
\hline \multirow[t]{3}{*}{$\mathrm{S}_{5}$} & 4.2602 & 291.03 & 0.0007 & HOMO-6 $\rightarrow$ LUMO & -0.25466 \\
\hline & & & & HOMO-4 $\rightarrow$ LUMO & -0.37842 \\
\hline & & & & HOMO-2 $\rightarrow$ LUMO & 0.52413 \\
\hline \multirow[t]{5}{*}{$\mathrm{S}_{6}$} & 4.4467 & 278.82 & 0.0010 & HOMO-6 $\rightarrow$ LUMO & 0.17410 \\
\hline & & & & HOMO-4 $\rightarrow$ LUMO & 0.20139 \\
\hline & & & & HOMO-2 $\rightarrow$ LUMO & 0.21361 \\
\hline & & & & $\mathrm{HOMO} \rightarrow \mathrm{LUMO}+3$ & 0.55002 \\
\hline & & & & HOMO $\rightarrow$ LUMO+5 & -0.25709 \\
\hline
\end{tabular}

Table S17: Calculated TD-DFT transitions of compound $\mathbf{5 j}$ at CAM-B3LYP/6-31+G(d) level.

\begin{tabular}{|c|c|c|c|c|c|}
\hline$\overline{S_{n}}$ & $E(e V)$ & $\lambda(\mathrm{nm})$ & $f$ & Configuration & $\mathrm{Cl}$ coefficient \\
\hline \multirow[t]{3}{*}{$\overline{S_{1}}$} & 3.8785 & 319.67 & 0.0526 & HOMO-2 $\rightarrow$ LUMO & -0.27422 \\
\hline & & & & HOMO-1 $\rightarrow$ LUMO & 0.20169 \\
\hline & & & & HOMO $\rightarrow$ LUMO+1 & 0.60273 \\
\hline \multirow[t]{3}{*}{$\mathrm{S}_{2}$} & 3.8789 & 319.63 & 0.3439 & HOMO-2 $\rightarrow$ LUMO+1 & 0.16699 \\
\hline & & & & HOMO-1 $\rightarrow$ LUMO+1 & -0.11515 \\
\hline & & & & HOMO $\rightarrow$ LUMO & 0.67349 \\
\hline \multirow[t]{4}{*}{$\mathrm{S}_{3}$} & 4.4668 & 277.57 & 0.1793 & HOMO-1 $\rightarrow$ LUMO & 0.29400 \\
\hline & & & & HOMO $\rightarrow$ LUMO+1 & -0.11438 \\
\hline & & & & $\mathrm{HOMO} \rightarrow$ LUMO+2 & 0.57525 \\
\hline & & & & $\mathrm{HOMO} \rightarrow$ LUMO+8 & 0.16383 \\
\hline $\mathrm{S}_{4}$ & 4.7557 & 260.70 & 0.0000 & HOMO-6 $\rightarrow$ LUMO & 0.15093 \\
\hline
\end{tabular}




\begin{tabular}{|c|c|c|c|c|c|}
\hline & & & & HOMO-4 $\rightarrow$ LUMO & 0.58703 \\
\hline & & & & HOMO-4 $\rightarrow$ LUMO+10 & 0.17017 \\
\hline & & & & HOMO-3 $\rightarrow$ LUMO & -0.26960 \\
\hline \multirow[t]{5}{*}{$\mathrm{S}_{5}$} & 4.7966 & 258.49 & 0.1729 & HOMO-7 $\rightarrow$ LUMO & 0.19115 \\
\hline & & & & HOMO-2 $\rightarrow$ LUMO & 0.18154 \\
\hline & & & & HOMO-1 $\rightarrow$ LUMO & 0.54089 \\
\hline & & & & HOMO $\rightarrow$ LUMO+1 & -0.10361 \\
\hline & & & & $\mathrm{HOMO} \rightarrow \mathrm{LUMO}+2$ & -0.29637 \\
\hline \multirow[t]{4}{*}{$\mathrm{S}_{6}$} & 4.9751 & 249.21 & 0.4849 & HOMO-7 $\rightarrow$ LUMO & 0.15506 \\
\hline & & & & HOMO-2 $\rightarrow$ LUMO & 0.56851 \\
\hline & & & & HOMO-1 $\rightarrow$ LUMO & -0.13808 \\
\hline & & & & $\mathrm{HOMO} \rightarrow$ LUMO+1 & 0.30769 \\
\hline
\end{tabular}

Table S18: Calculated TD-DFT transitions of compound $5 \mathrm{~m}$ at B3LYP/6-31+G(d) level (IEFPCM).

\begin{tabular}{|c|c|c|c|c|c|}
\hline$S_{n}$ & $E(e V)$ & $\lambda(\mathrm{nm})$ & $f$ & Configuration & $\mathrm{Cl}$ coefficient \\
\hline \multirow[t]{3}{*}{$S_{1}$} & 3.3604 & 368.96 & 0.3442 & HOMO-3 $\rightarrow$ LUMO+1 & 0.12728 \\
\hline & & & & HOMO-2 $\rightarrow$ LUMO & -0.10811 \\
\hline & & & & HOMO $\rightarrow$ LUMO & 0.68315 \\
\hline \multirow[t]{3}{*}{$\mathrm{S}_{2}$} & 3.5081 & 353.42 & 0.0041 & HOMO-3 $\rightarrow$ LUMO & -0.15145 \\
\hline & & & & HOMO-1 $\rightarrow$ LUMO & 0.53386 \\
\hline & & & & HOMO $\rightarrow$ LUMO+1 & 0.42913 \\
\hline \multirow[t]{3}{*}{$\mathrm{S}_{3}$} & 3.5900 & 345.36 & 0.3606 & HOMO-3 $\rightarrow$ LUMO & -0.17819 \\
\hline & & & & HOMO-1 $\rightarrow$ LUMO & -0.45711 \\
\hline & & & & HOMO $\rightarrow$ LUMO+1 & 0.50413 \\
\hline \multirow[t]{2}{*}{$\mathrm{S}_{4}$} & 3.9203 & 316.26 & 0.0345 & HOMO-2 $\rightarrow$ LUMO & 0.67737 \\
\hline & & & & HOMO-1 $\rightarrow$ LUMO+1 & -0.10023 \\
\hline \multirow[t]{2}{*}{$\mathrm{S}_{5}$} & 4.0336 & 307.38 & 0.0802 & HOMO-3 $\rightarrow$ LUMO & 0.12767 \\
\hline & & & & $\mathrm{HOMO} \rightarrow$ LUMO+2 & 0.68239 \\
\hline $\mathrm{S}_{6}$ & 4.1547 & 298.42 & 0.0112 & HOMO-1 $\rightarrow$ LUMO+1 & 0.69127 \\
\hline
\end{tabular}

Table S19: Calculated TD-DFT transitions of compound 5m at CAM-B3LYP/6-31+G(d) level.

\begin{tabular}{cccclr}
\hline $\mathrm{S}_{\mathrm{n}}$ & $\mathrm{E}(\mathrm{eV})$ & $\lambda(\mathrm{nm})$ & $f$ & Configuration & Cl coefficient \\
\hline $\mathrm{S}_{1}$ & 3.8455 & 322.41 & 0.3480 & HOMO-3 $\rightarrow$ LUMO+1 & 0.18371 \\
& & & & HOMO $\rightarrow$ LUMO & 0.66728 \\
\hline
\end{tabular}




\begin{tabular}{|c|c|c|c|c|c|}
\hline \multirow[t]{4}{*}{$\overline{S_{2}}$} & 3.8573 & 321.43 & 0.0642 & HOMO-3 $\rightarrow$ LUMO & -0.30398 \\
\hline & & & & HOMO-2 $\rightarrow$ LUMO+1 & 0.11319 \\
\hline & & & & HOMO-1 $\rightarrow$ LUMO & -0.13477 \\
\hline & & & & HOMO $\rightarrow$ LUMO+1 & 0.59525 \\
\hline \multirow[t]{4}{*}{$\mathrm{S}_{3}$} & 4.3703 & 283.70 & 0.3965 & HOMO-7 $\rightarrow$ LUMO & -0.15611 \\
\hline & & & & HOMO-1 $\rightarrow$ LUMO & 0.48862 \\
\hline & & & & $\mathrm{HOMO} \rightarrow$ LUMO+1 & 0.14544 \\
\hline & & & & $\mathrm{HOMO} \rightarrow \mathrm{LUMO}+2$ & 0.42451 \\
\hline \multirow[t]{4}{*}{$\mathrm{S}_{4}$} & 4.6510 & 266.57 & 0.0426 & HOMO-7 $\rightarrow$ LUMO & 0.11904 \\
\hline & & & & HOMO-2 $\rightarrow$ LUMO+2 & 0.13456 \\
\hline & & & & HOMO-1 $\rightarrow$ LUMO & -0.41490 \\
\hline & & & & $\mathrm{HOMO} \rightarrow$ LUMO+2 & 0.48951 \\
\hline \multirow[t]{3}{*}{$\mathrm{S}_{5}$} & 4.7641 & 260.25 & 0.0018 & HOMO-6 $\rightarrow$ LUMO & 0.55422 \\
\hline & & & & HOMO-2 $\rightarrow$ LUMO & -0.34812 \\
\hline & & & & HOMO-1 $\rightarrow$ LUMO+2 & 0.10241 \\
\hline \multirow[t]{7}{*}{$\mathrm{S}_{6}$} & 4.9404 & 250.96 & 0.3414 & HOMO-7 $\rightarrow$ LUMO & 0.14963 \\
\hline & & & & HOMO-3 $\rightarrow$ LUMO & 0.44687 \\
\hline & & & & HOMO-2 $\rightarrow$ LUMO+4 & -0.12557 \\
\hline & & & & HOMO-1 $\rightarrow$ LUMO+2 & 0.10180 \\
\hline & & & & HOMO-1 $\rightarrow$ LUMO+3 & 0.17585 \\
\hline & & & & HOMO $\rightarrow$ LUMO+1 & 0.24614 \\
\hline & & & & $\mathrm{HOMO} \rightarrow$ LUMO+4 & 0.19211 \\
\hline
\end{tabular}

Table S20: Calculated TD-DFT transitions of compound $\mathbf{5 n}$ at B3LYP/6-31+G(d) level (IEFPCM).

\begin{tabular}{cccclr}
\hline $\mathrm{S}_{\mathrm{n}}$ & $\mathrm{E}(\mathrm{eV})$ & $\lambda(\mathrm{nm})$ & $f$ & Configuration & Cl coefficient \\
\hline $\mathrm{S}_{1}$ & 2.8937 & 428.46 & 0.1861 & HOMO-1 $\rightarrow$ LUMO & 0.70223 \\
$\mathrm{~S}_{2}$ & 2.9207 & 424.50 & 0.1420 & $\mathrm{HOMO}-2 \rightarrow$ LUMO & -0.11588 \\
& & & & HOMO $\rightarrow$ LUMO & 0.69170 \\
$\mathrm{~S}_{3}$ & 3.3347 & \multirow{3}{*}{371.80} & 0.1917 & HOMO-3 $\rightarrow$ LUMO & 0.12492 \\
& & & & HOMO-2 $\rightarrow$ LUMO+1 & 0.10699 \\
& & & & HOMO $\rightarrow$ LUMO+1 & 0.68451 \\
$\mathrm{~S}_{4}$ & 3.4689 & \multirow{3}{*}{357.42} & 0.0115 & HOMO-2 $\rightarrow$ LUMO & 0.16461 \\
& & & & HOMO- $\rightarrow$ LUMO+1 & 0.68360 \\
$\mathrm{~S}_{5}$ & 3.6283 & \multirow{2}{*}{341.72} & 0.2148 & HOMO-3 $\rightarrow$ LUMO+1 & -0.14721 \\
\hline
\end{tabular}




\begin{tabular}{|c|c|c|c|c|c|}
\hline & & & & HOMO-2 $\rightarrow$ LUMO & 0.65264 \\
\hline & & & & HOMO-1 $\rightarrow$ LUMO+1 & -0.17062 \\
\hline & & & & HOMO $\rightarrow$ LUMO & 0.10362 \\
\hline \multirow[t]{4}{*}{$\mathrm{S}_{6}$} & 3.8551 & 321.61 & 0.0717 & HOMO-3 $\rightarrow$ LUMO & 0.23736 \\
\hline & & & & HOMO-2 $\rightarrow$ LUMO+1 & 0.57136 \\
\hline & & & & HOMO $\rightarrow$ LUMO+1 & -0.14661 \\
\hline & & & & HOMO $\rightarrow$ LUMO+2 & -0.29782 \\
\hline
\end{tabular}

Table S21: Calculated TD-DFT transitions of compound 5n at CAM-B3LYP/6-31+G(d) level.

\begin{tabular}{|c|c|c|c|c|c|}
\hline$S_{n}$ & $E(e V)$ & $\lambda(\mathrm{nm})$ & $f$ & Configuration & $\mathrm{Cl}$ coefficient \\
\hline \multirow[t]{3}{*}{$\overline{S_{1}}$} & 3.7682 & 329.03 & 0.3605 & HOMO-3 $\rightarrow$ LUMO+1 & 0.17271 \\
\hline & & & & HOMO-2 $\rightarrow$ LUMO & 0.16243 \\
\hline & & & & HOMO $\rightarrow$ LUMO & 0.65687 \\
\hline \multirow[t]{4}{*}{$\mathrm{S}_{2}$} & 3.8216 & 324.43 & 0.0471 & HOMO-3 $\rightarrow$ LUMO & -0.30443 \\
\hline & & & & HOMO-2 $\rightarrow$ LUMO+1 & 0.27446 \\
\hline & & & & HOMO-1 $\rightarrow$ LUMO & -0.16663 \\
\hline & & & & HOMO $\rightarrow$ LUMO+1 & 0.53857 \\
\hline \multirow[t]{4}{*}{$\mathrm{S}_{3}$} & 4.0469 & 306.37 & 0.5868 & HOMO-6 $\rightarrow$ LUMO & -0.15368 \\
\hline & & & & HOMO-1 $\rightarrow$ LUMO & 0.59995 \\
\hline & & & & HOMO $\rightarrow$ LUMO+1 & 0.19063 \\
\hline & & & & $\mathrm{HOMO} \rightarrow$ LUMO+2 & 0.22921 \\
\hline \multirow[t]{5}{*}{$\mathrm{S}_{4}$} & 4.4588 & 278.06 & 0.0011 & HOMO-7 $\rightarrow$ LUMO & -0.11661 \\
\hline & & & & HOMO-3 $\rightarrow$ LUMO+1 & 0.10153 \\
\hline & & & & HOMO-2 $\rightarrow$ LUMO & 0.60325 \\
\hline & & & & HOMO-1 $\rightarrow$ LUMO+2 & -0.21902 \\
\hline & & & & HOMO $\rightarrow$ LUMO & -0.15586 \\
\hline \multirow[t]{5}{*}{$\mathrm{S}_{5}$} & 4.5318 & 273.59 & 0.0288 & HOMO-2 $\rightarrow$ LUMO+2 & 0.20168 \\
\hline & & & & HOMO-1 $\rightarrow$ LUMO & -0.21196 \\
\hline & & & & HOMO-1 $\rightarrow$ LUMO+5 & -0.15956 \\
\hline & & & & $\mathrm{HOMO} \rightarrow$ LUMO+2 & 0.53205 \\
\hline & & & & $\mathrm{HOMO} \rightarrow \mathrm{LUMO}+3$ & -0.13371 \\
\hline \multirow[t]{3}{*}{$\mathrm{S}_{6}$} & 4.6042 & 269.28 & 0.0373 & HOMO-2 $\rightarrow$ LUMO & -0.11919 \\
\hline & & & & HOMO-2 $\rightarrow$ LUMO+5 & -0.18637 \\
\hline & & & & HOMO-2 $\rightarrow$ LUMO+8 & 0.11108 \\
\hline
\end{tabular}




\begin{tabular}{llr}
\hline HOMO-1 $\rightarrow$ LUMO+1 & 0.14313 \\
HOMO $1 \rightarrow$ LUMO+2 & -0.11112 \\
HOMO-1 $\rightarrow$ LUMO+6 & 0.36931 \\
HOMO $-1 \rightarrow$ LUMO+7 & 0.17026 \\
HOMO $\rightarrow$ LUMO+5 & 0.34771 \\
HOMO $\rightarrow$ LUMO+8 & -0.16636 \\
\hline
\end{tabular}

Table S22: Calculated TD-DFT transitions of compound $\mathbf{5 0}$ at B3LYP/6-31+G(d) level (IEFPCM).

\begin{tabular}{|c|c|c|c|c|c|}
\hline $\mathrm{S}_{\mathrm{n}}$ & $E(e V)$ & $\lambda(\mathrm{nm})$ & $f$ & Configuration & $\mathrm{Cl}$ coefficient \\
\hline \multirow[t]{2}{*}{$\overline{S_{1}}$} & 3.4390 & 360.52 & 0.3746 & HOMO-4 $\rightarrow$ LUMO+1 & -0.13472 \\
\hline & & & & HOMO $\rightarrow$ LUMO & 0.68587 \\
\hline \multirow[t]{3}{*}{$\mathrm{S}_{2}$} & 3.5766 & 346.65 & 0.0719 & HOMO-4 $\rightarrow$ LUMO & 0.21401 \\
\hline & & & & HOMO-1 $\rightarrow$ LUMO & 0.20601 \\
\hline & & & & $\mathrm{HOMO} \rightarrow \mathrm{LUMO}+1$ & 0.63123 \\
\hline \multirow[t]{3}{*}{$\mathrm{S}_{3}$} & 3.8309 & 323.64 & 0.2402 & HOMO-4 $\rightarrow$ LUMO & -0.11091 \\
\hline & & & & HOMO-1 $\rightarrow$ LUMO & 0.67035 \\
\hline & & & & HOMO $\rightarrow$ LUMO+1 & -0.18152 \\
\hline $\mathrm{S}_{4}$ & 4.0647 & 305.02 & 0.0054 & HOMO-2 $\rightarrow$ LUMO & 0.68545 \\
\hline \multirow[t]{3}{*}{$\mathrm{S}_{5}$} & 4.1115 & 301.56 & 0.0993 & HOMO-4 $\rightarrow$ LUMO & 0.18774 \\
\hline & & & & $\mathrm{HOMO} \rightarrow \mathrm{LUMO}+2$ & 0.65477 \\
\hline & & & & $\mathrm{HOMO} \rightarrow \mathrm{LUMO}+4$ & -0.14476 \\
\hline \multirow[t]{2}{*}{$\mathrm{S}_{6}$} & 4.2697 & 290.38 & 0.0628 & HOMO-4 $\rightarrow$ LUMO & 0.12620 \\
\hline & & & & HOMO-3 $\rightarrow$ LUMO & 0.68576 \\
\hline
\end{tabular}

Table S23: Calculated TD-DFT transitions of compound $\mathbf{5 o}$ at CAM-B3LYP/6-31+G(d) level.

\begin{tabular}{cccclr}
\hline $\mathrm{S}_{\mathrm{n}}$ & $\mathrm{E}(\mathrm{eV})$ & $\lambda(\mathrm{nm})$ & $f$ & Configuration & Cl coefficient \\
\hline $\mathrm{S}_{1}$ & 3.8711 & 320.28 & 0.3402 & $\mathrm{HOMO}-3 \rightarrow$ LUMO+1 & 0.16512 \\
& & & & HOMO-1 $\rightarrow$ LUMO+1 & -0.10698 \\
& & & & HOMO $\rightarrow$ LUMO & 0.67378 \\
$\mathrm{~S}_{2}$ & 3.8752 & 319.94 & 0.0528 & HOMO-3 $\rightarrow$ LUMO & -0.27133 \\
& & & & HOMO-1 $\rightarrow$ LUMO & 0.19036 \\
& & & & HOMO $\rightarrow$ LUMO+1 & 0.60012 \\
$\mathrm{~S}_{3}$ & 4.4748 & 277.07 & 0.2849 & HOMO-7 $\rightarrow$ LUMO & -0.11114 \\
& & & HOMO- $\rightarrow$ LUMO & -0.42381 \\
& & & &
\end{tabular}




\begin{tabular}{|c|c|c|c|c|c|}
\hline & & & & $\mathrm{HOMO} \rightarrow \mathrm{LUMO}+1$ & 0.16881 \\
\hline & & & & $\mathrm{HOMO} \rightarrow \mathrm{LUMO}+2$ & 0.45840 \\
\hline & & & & $\mathrm{HOMO} \rightarrow \mathrm{LUMO}+4$ & -0.21480 \\
\hline \multirow[t]{4}{*}{$\mathrm{S}_{4}$} & 4.7539 & 260.81 & 0.0000 & HOMO-6 $\rightarrow$ LUMO & 0.58724 \\
\hline & & & & HOMO-6 $\rightarrow$ LUMO+9 & -0.16696 \\
\hline & & & & HOMO-5 $\rightarrow$ LUMO & -0.24091 \\
\hline & & & & HOMO-2 $\rightarrow$ LUMO & 0.19484 \\
\hline \multirow[t]{6}{*}{$\mathrm{S}_{5}$} & 4.7626 & 260.33 & 0.0536 & HOMO-7 $\rightarrow$ LUMO & 0.13037 \\
\hline & & & & HOMO-4 $\rightarrow$ LUMO & -0.10399 \\
\hline & & & & HOMO-3 $\rightarrow$ LUMO & 0.17775 \\
\hline & & & & HOMO-1 $\rightarrow$ LUMO & 0.46254 \\
\hline & & & & $\mathrm{HOMO} \rightarrow \mathrm{LUMO}+2$ & 0.38356 \\
\hline & & & & $\mathrm{HOMO} \rightarrow$ LUMO+4 & -0.20558 \\
\hline \multirow[t]{6}{*}{$\mathrm{S}_{6}$} & 4.9752 & 249.20 & 0.5029 & HOMO-7 $\rightarrow$ LUMO & 0.13423 \\
\hline & & & & HOMO-4 $\rightarrow$ LUMO & -0.20104 \\
\hline & & & & HOMO-3 $\rightarrow$ LUMO & 0.53315 \\
\hline & & & & HOMO-1 $\rightarrow$ LUMO & -0.13643 \\
\hline & & & & $\mathrm{HOMO} \rightarrow \mathrm{LUMO}+1$ & 0.30416 \\
\hline & & & & $\mathrm{HOMO} \rightarrow \mathrm{LUMO}+2$ & -0.11881 \\
\hline
\end{tabular}

Table S24: Calculated TD-DFT Emission of compound $5 n$ at B3LYP/6-31+G(d) level (IEFPCM).

\begin{tabular}{cccclr}
\hline $\mathrm{S}_{\mathrm{n}}$ & $\mathrm{E}(\mathrm{eV})$ & $\lambda(\mathrm{nm})$ & $f$ & Configuration & Cl coefficient \\
\hline $\mathrm{S}_{1}$ & 2.4974 & 496.45 & 0.2906 & HOMO-1 $\rightarrow$ LUMO & 0.14663 \\
& & & & HOMO $\rightarrow$ LUMO & -0.68616 \\
& & & & & \\
\hline
\end{tabular}

${ }^{1} \mathrm{H}$-, ${ }^{19} \mathrm{~F}$-, and ${ }^{13} \mathrm{C}$-NMR Spectra 


\section{4-chloro-3,5-bis(phenylethynyl)pyridine (2a)}

\begin{tabular}{lll}
\multicolumn{1}{c}{ Parameter } & \multicolumn{1}{c}{ Value } \\
1 Solvent & CDCl3 \\
2 Temperature & 298.2 \\
3 Pulse Sequence & 2930 \\
4 Number of Scans & 16 \\
5 Receiver Gain & 256 \\
6 Relaxation Delay & 1.0000 \\
7 Pulse Width & 10.0000 \\
8 Acquisition Time & 5.2954 \\
9 Spectrometer Frequency 300.13 \\
10 Spectral Width & 6188.1 \\
11 Lowest Frequency & -1245.4 \\
12 Nucleus & $1 \mathrm{H}$ \\
13 Acquired Size & 32768 \\
14 Spectral Size & 32768
\end{tabular}
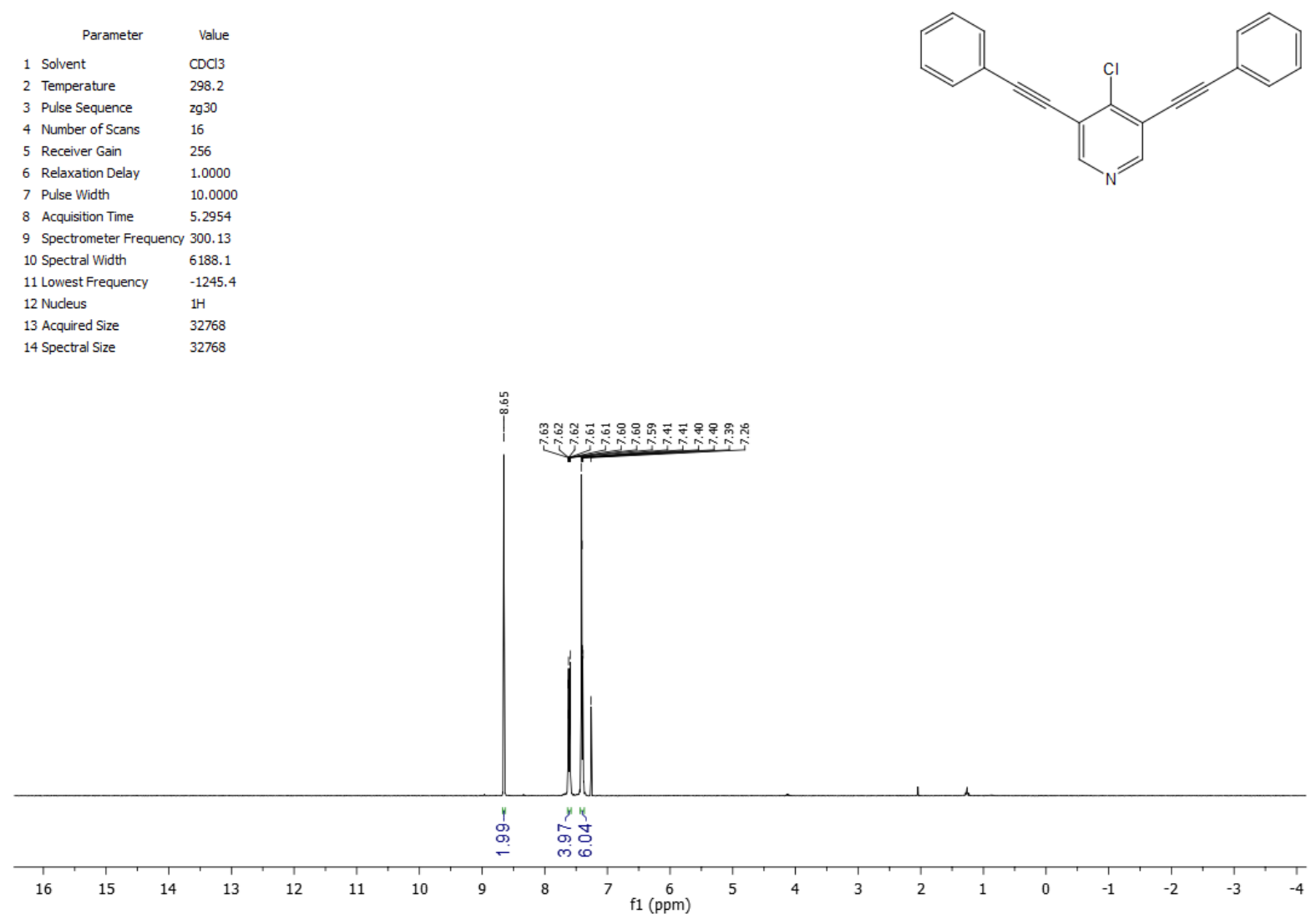

\begin{tabular}{lll}
\multicolumn{1}{c}{ Parameter } & \multicolumn{1}{c}{ Value } \\
1 Solvent & CDCl3 \\
2 Temperature & 298.5 \\
3 Pulse Sequence & $29 \mathrm{gg} 30$ \\
4 Number of Scans & 1024 \\
5 Receiver Gain & 2050 \\
6 Relaxation Delay & 2.0000 \\
7 Pulse Width & 10.0000 \\
8 Acquisition Time & 1.8176 \\
9 Spectrometer Frequency & 75.47 \\
10 Spectral Width & 18028.8 \\
11 Lowest Frequency & -1469.6 \\
12 Nucleus & $13 \mathrm{C}$ \\
13 Acquired Size & 32768 \\
14 Spectral Size & 32768
\end{tabular}

14 Spectral Size $\quad 32768$

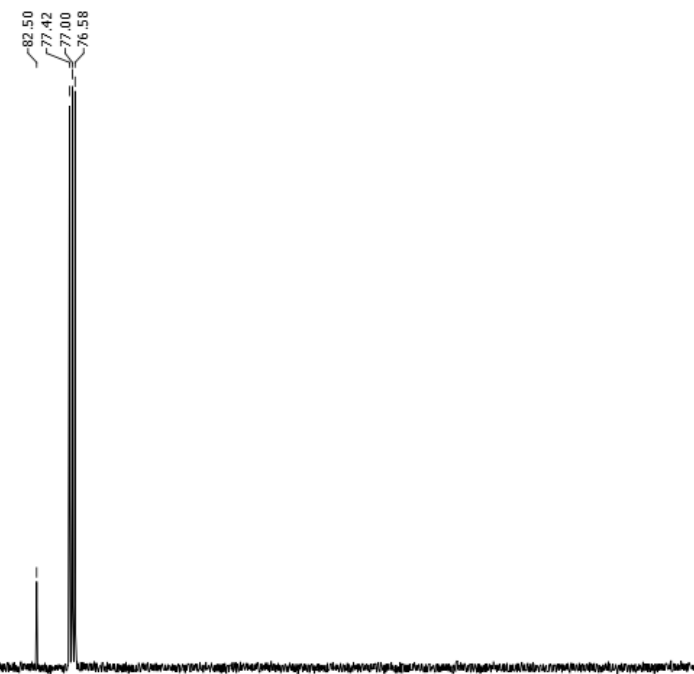


4-chloro-3,5-bis(p-tolylethynyl)pyridine (2b)

\begin{tabular}{lll}
\multicolumn{1}{c}{ Parameter } & \multicolumn{1}{c}{ Value } \\
1 Solvent & CDCl3 \\
2 Temperature & 298.0 \\
3 & Pulse Sequence & 2930 \\
4 Number of Scans & 16 \\
5 5 & Receiver Gain & 63 \\
6 Relaxation Delay & 1.0000 \\
7 Pulse Width & 8.0000 \\
8 Acquisition Time & 3.2768 \\
9 Spectrometer Frequency 500.13 \\
10 Spectral Width & 10000.0 \\
11 Lowest Frequency & -1924.1 \\
12 Nucleus & $1 \mathrm{H}$ \\
13 Acquired Size & 32768 \\
14 Spectral Size & 65536
\end{tabular}
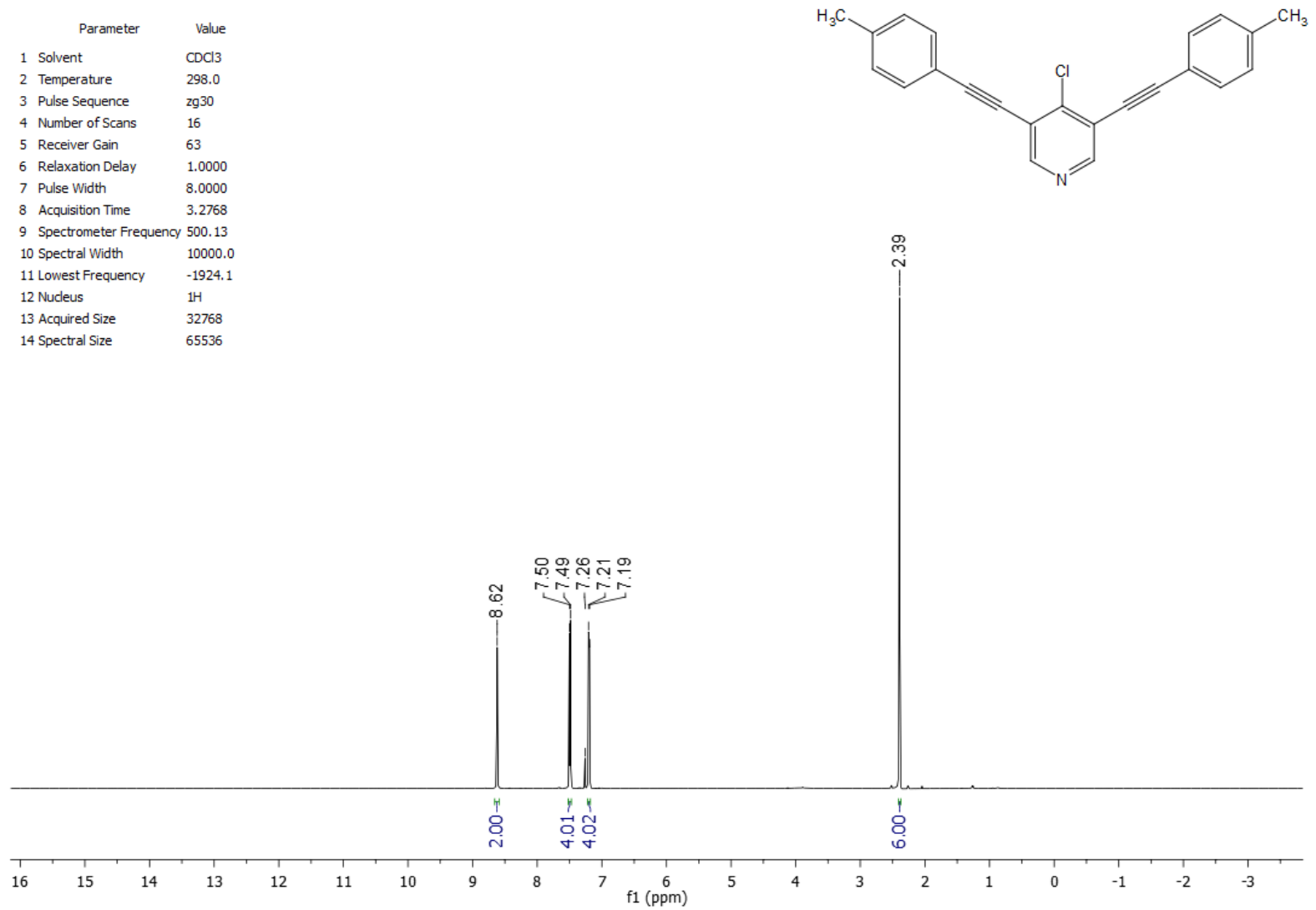

\begin{tabular}{lll}
\multicolumn{1}{c}{ Parameter } & \multicolumn{1}{c}{ Value } \\
1 Solvent & CDCl3 \\
2 2emperature & 298.1 \\
3 Pulse Sequence & $29 p 930$ \\
4 Number of Scans & 1024 \\
5 Receiver Gain & 101 \\
6 Relaxation Delay & 2.0000 \\
7 Pulse Width & 10.0000 \\
8 Acquisition Time & 1.0879 \\
9 Spectrometer Frequency & 125.76 \\
10 Spectral Width & 30120.5 \\
11 Lowest Frequency & -2491.0 \\
12 Nucleus & $13 \mathrm{C}$ \\
13 Acquired Size & 32768 \\
14 Spectral Size & 32768
\end{tabular}
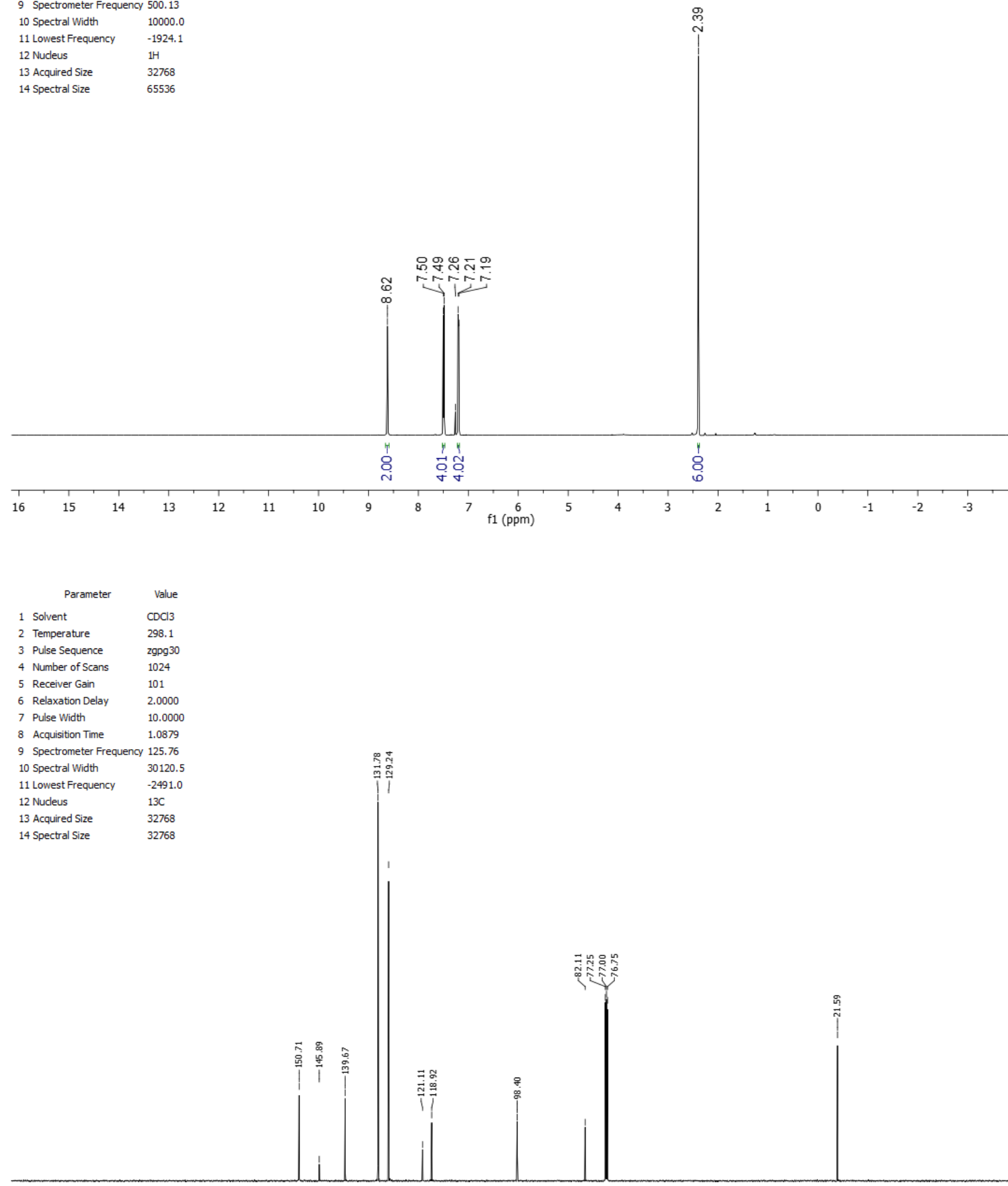

$\begin{array}{lllllllllllllllllllllll}210 & 200 & 190 & 180 & 170 & 160 & 150 & 140 & 130 & 120 & 110 & 100 & 90 & 80 & 70 & 60 & 50 & 40 & 30 & 20 & 10 & 0 & -10\end{array}$ 
4-chloro-3,5-bis( $m$-tolylethynyl)pyridine (2c)

\begin{tabular}{lll} 
& \multicolumn{1}{c}{ Parameter } & \multicolumn{1}{c}{ Value } \\
1 Solvent & CDCl3 \\
2 2 & Temperature & 298.2 \\
3 & Pulse Sequence & 2930 \\
4 & Number of Scans & 16 \\
5 & Receiver Gain & 63 \\
6 & Relaxation Delay & 1.0000 \\
7 Pulse Width & 8.0000 \\
8 & Acquisition Time & 3.2768 \\
9 & Spectrometer Frequency & 500.13 \\
10 & Spectral Width & 10000.0 \\
11 Lowest Frequency & -1923.9 \\
12 Nudeus & $1 \mathrm{H}$ \\
13 Acquired Size & 32768 \\
14 Spectral Size & 65536
\end{tabular}
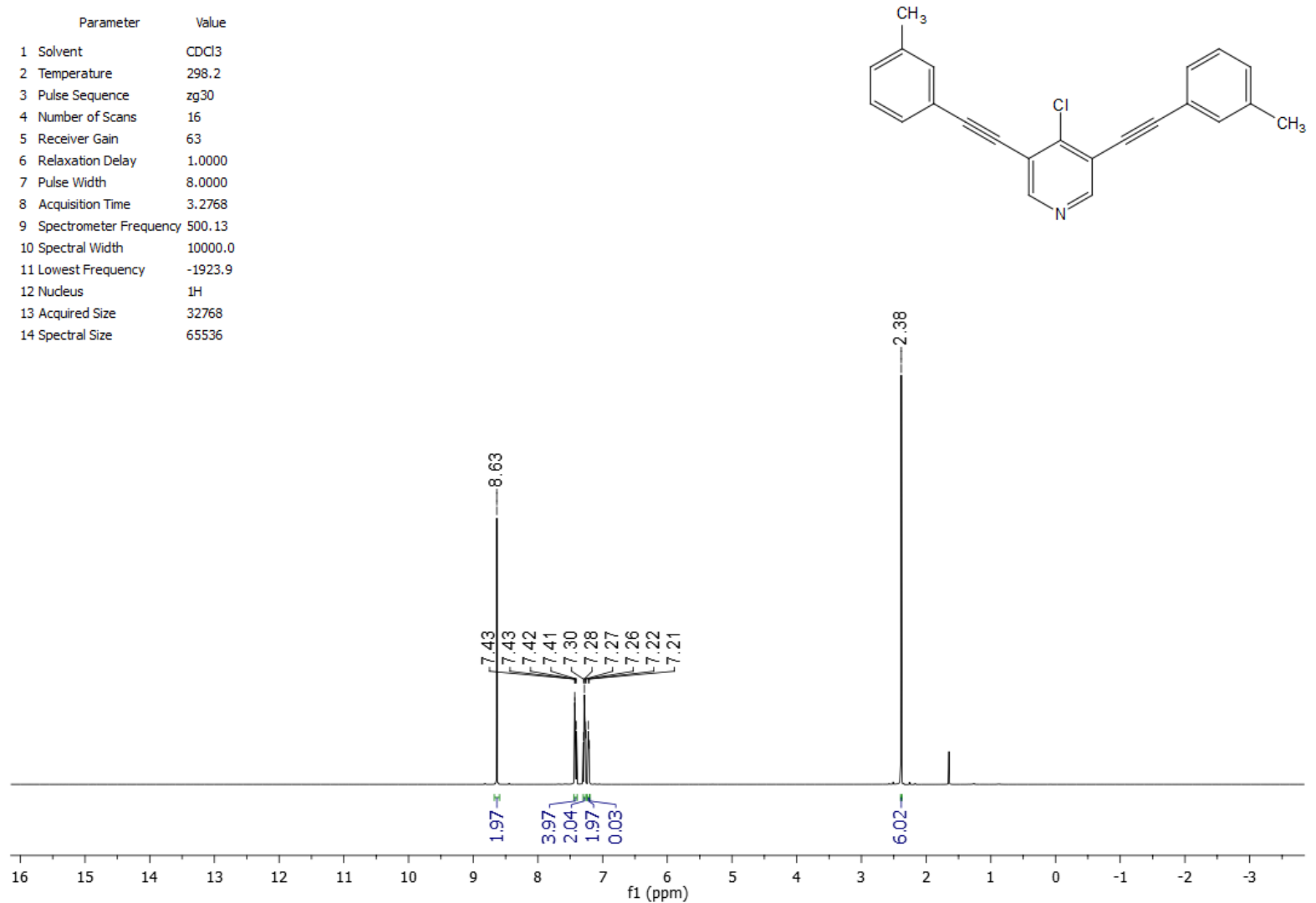

\begin{tabular}{lll}
\multicolumn{1}{c}{ Parameter } & \multicolumn{1}{c}{ Value } \\
1 Solvent & CDCl3 \\
2 2emperature & 298.1 \\
3 Pulse Sequence & $29 \mathrm{~g} 930$ \\
4 N & Number of Scans & 2048 \\
5 & Receiver Gain & 101 \\
6 Relaxation Delay & 2.0000 \\
7 Pulse Width & 10.0000 \\
8 Acquisition Time & 1.0879 \\
9 Spectrometer Frequency & 125.76 \\
10 Spectral Width & 30120.5 \\
11 Lowest Frequency & -2489.7 \\
12 Nudeus & $13 \mathrm{C}$ \\
13 Acquired Size & 32768 \\
14 Spectral Size & 32768
\end{tabular}

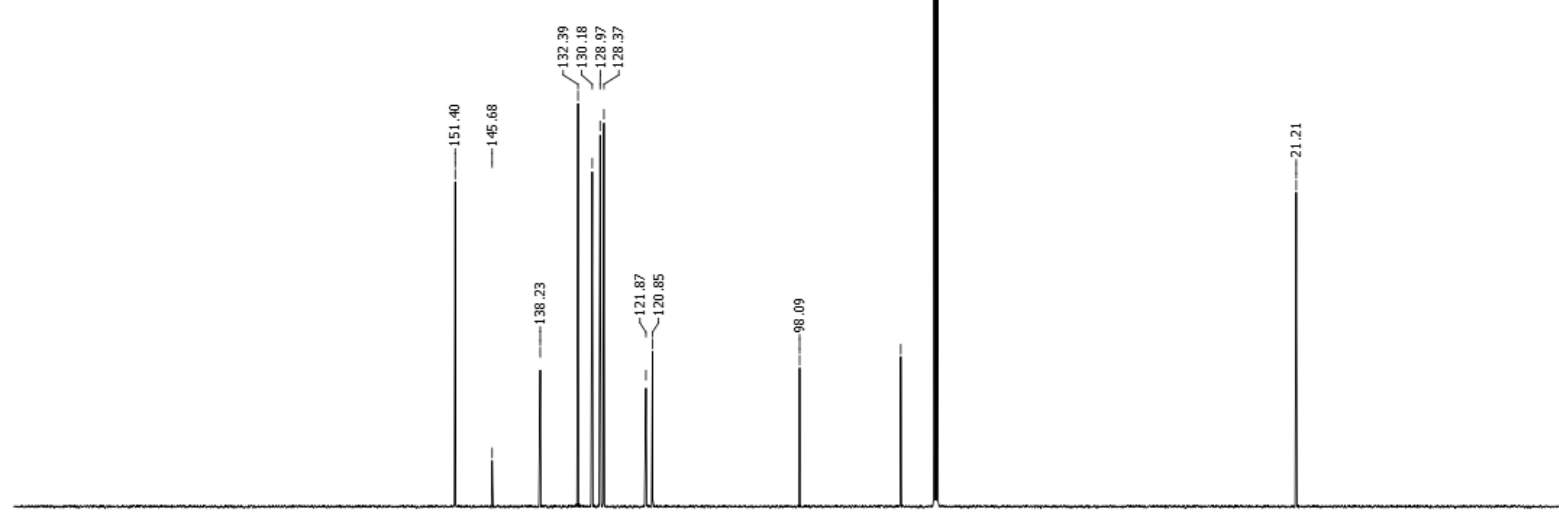

$\begin{array}{lllllllllllllllllllllll}210 & 200 & 190 & 180 & 170 & 160 & 150 & 140 & 130 & 120 & 110 & \begin{array}{c}100 \\ \mathrm{f} 1(\mathrm{ppm})\end{array} & 90 & 80 & 70 & 60 & 50 & 40 & 30 & 20 & 10 & 0 & -10\end{array}$ 
4-chloro-3,5-bis((4-fluorophenyl)ethynyl)pyridine (2d)

\begin{tabular}{|c|c|c|}
\hline & Parameter & \\
\hline & Solvent & \\
\hline 2 & Temperature & \\
\hline 3 & Pulse Sequence & \\
\hline 4 & Number of Scans & \\
\hline & Receiver Gain & \\
\hline 6 & Relaxation Delay & \\
\hline & Pulse Width & \\
\hline & Acquisition Time & \\
\hline & Spectrometer Frequency & \\
\hline & Spectral Width & \\
\hline & 1 Lowest Frequency & \\
\hline & 2 Nucleus & \\
\hline & 3 Acquired Size & \\
\hline & Tpectral Size & \\
\hline
\end{tabular}
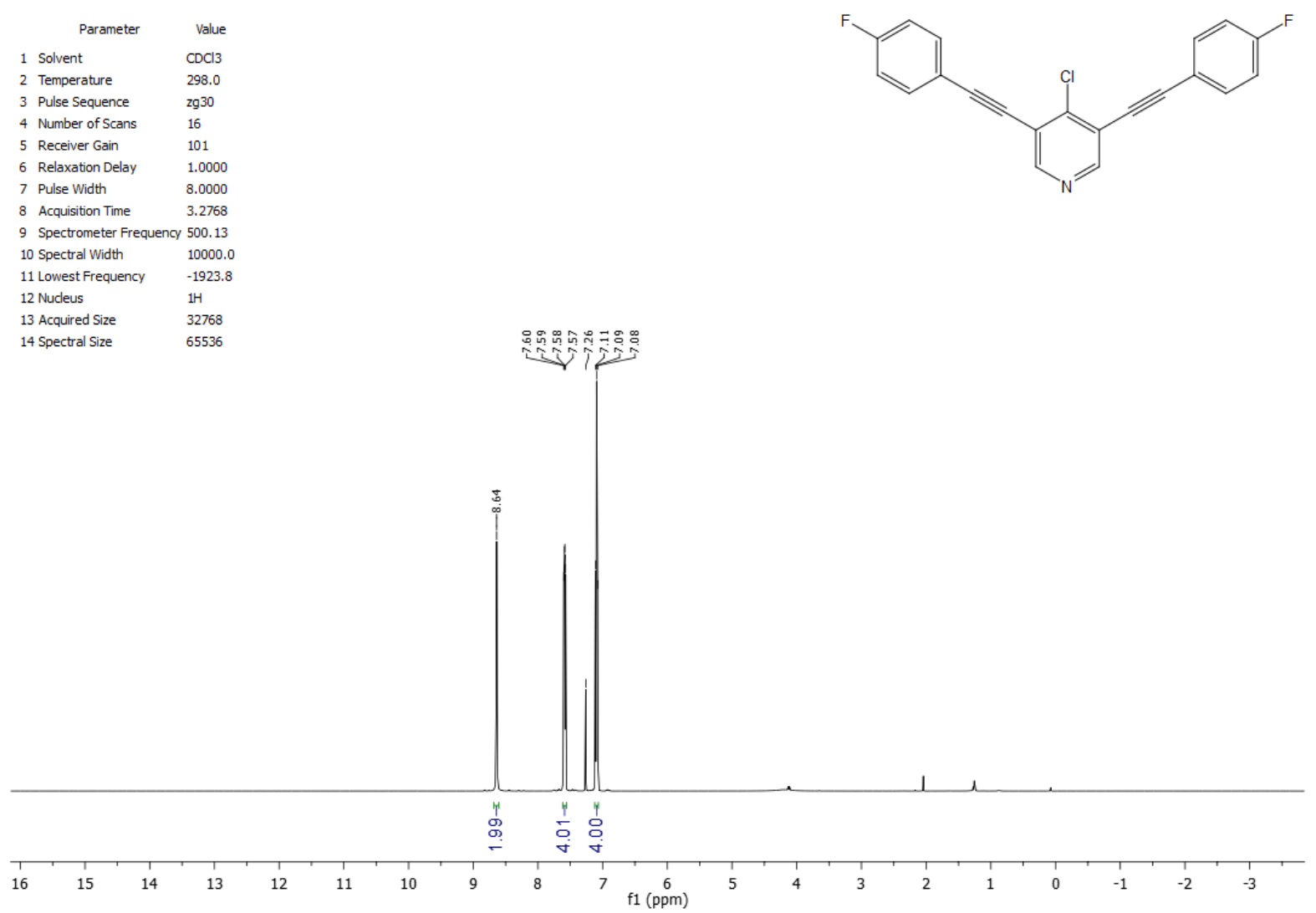

\begin{tabular}{lll}
\multicolumn{1}{c}{ Parameter } & \multicolumn{1}{c}{ Value } \\
1 Solvent & CDCl3 \\
2 2emperature & 298.1 \\
3 Pulse Sequence & zgig \\
4 Number of Scans & 16 \\
5 Receiver Gain & 101 \\
6 Relaxation Delay & 1.0000 \\
7 Pulse Width & 12.0000 \\
8 Acquisition Time & 0.5767 \\
9 Spectrometer Frequency 470.59 \\
10 Spectral Width & 113636.4 \\
11 Lowest Frequency & -103877.4 \\
12 Nudeus & $19 F$ \\
13 Acquired Size & 65536 \\
14 Spectral Size & 65536
\end{tabular}

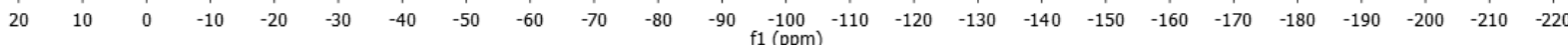




$$
\begin{array}{lll} 
& \multicolumn{1}{c}{\text { Parameter }} & \multicolumn{1}{c}{\text { Value }} \\
1 \text { Solvent } & \text { CDCl3 } \\
2 \text { Temperature } & 298.0 \\
3 \text { Pulse Sequence } & \text { 29pg } 30 \\
4 \text { Number of Scans } & 1024 \\
5 \text { Receiver Gain } & 101 \\
6 \text { Relaxation Delay } & 2.0000 \\
7 \text { Pulse Width } & 10.0000 \\
8 \text { Acquisition Time } & 1.0879 \\
9 \text { Spectrometer Frequency } & 125.76 \\
10 \text { Spectral Width } & 30120.5 \\
\text { 11 Lowest Frequency } & -2488.9 \\
\text { 12 Nucleus } & 13 \mathrm{C} \\
\text { 13 Acquired Size } & 32768 \\
14 \text { Spectral Size } & 32768
\end{array}
$$

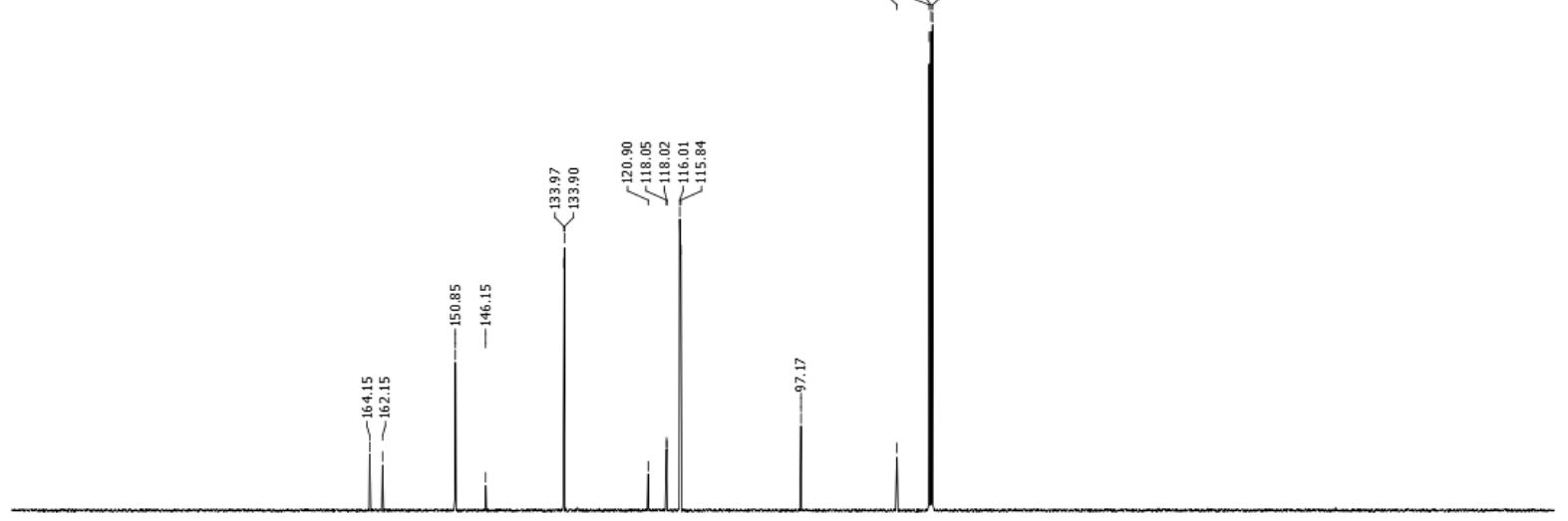

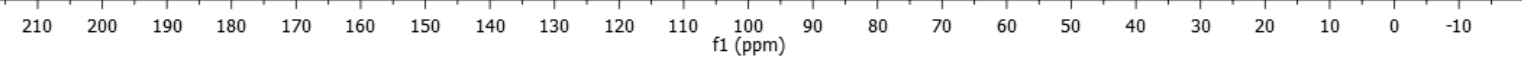


4-chloro-3,5-bis((4-(trifluoromethyl)phenyl)ethynyl)pyridine (ae)

\begin{tabular}{lll}
\multicolumn{1}{c}{ Parameter } & \multicolumn{1}{c}{ Value } \\
1 Solvent & $\mathrm{CDCl} 3$ \\
2 Temperature & 298.4 \\
3 Pulse Sequence & 2930 \\
4 Number of Scans & 16 \\
5 Receiver Gain & 812 \\
6 Relaxation Delay & 1.0000 \\
7 Pulse Width & 10.0000 \\
8 Acquisition Time & 6.3439 \\
9 Spectrometer Frequency & 250.13 \\
10 Spectral Width & 5165.3 \\
11 Lowest Frequency & -1038.6 \\
12 Nucleus & $1 \mathrm{H}$ \\
13 Acquired Size & 32768 \\
14 Spectral Size & 32768
\end{tabular}
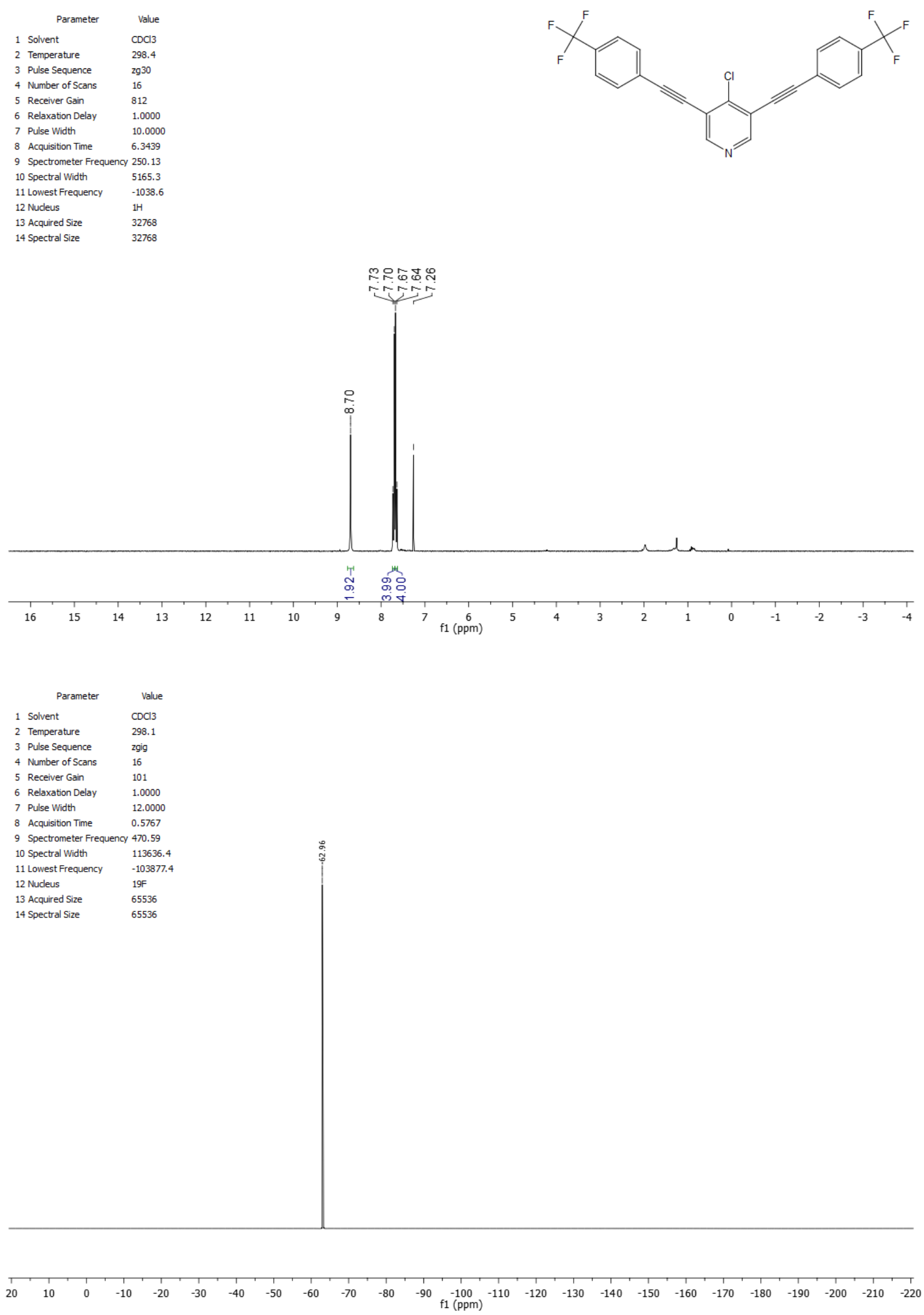

S50 


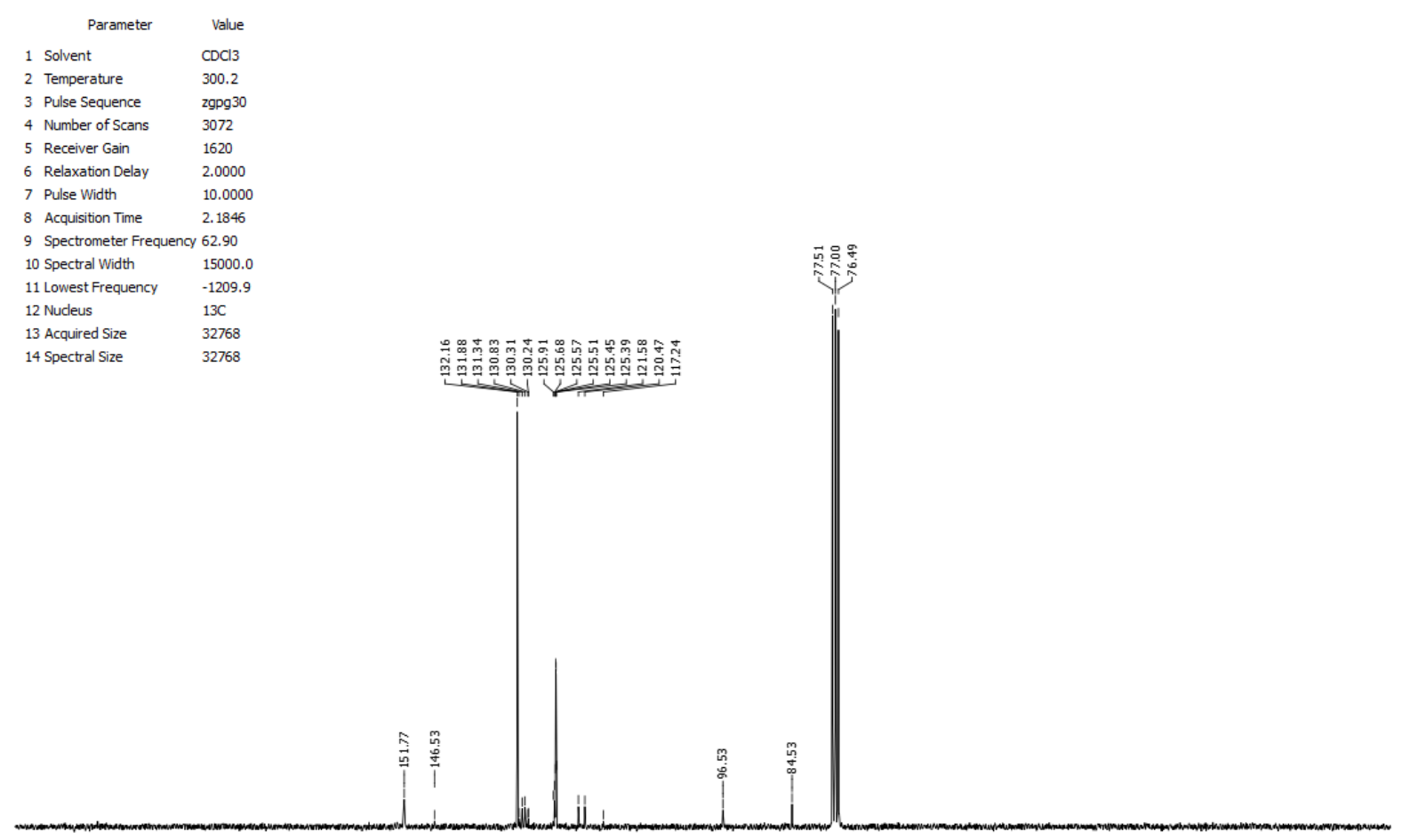

$\begin{array}{lllllllllllllllllllllllllll}210 & 200 & 190 & 180 & 170 & 160 & 150 & 140 & 130 & 120 & 110 & 100 & 90 & 80 & 70 & 60 & 50 & 40 & 30 & 20 & 10 & 0 & -10\end{array}$ 
4-chloro-3,5-bis((4-methoxyphenyl)ethynyl)pyridine (2f)

\begin{tabular}{lll} 
& \multicolumn{1}{c}{ Parameter } & \multicolumn{1}{c}{ Value } \\
1 Solvent & CDd3 \\
2 2 & Temperature & 298.2 \\
3 & Pulse Sequence & 2930 \\
4 & Number of Scans & 16 \\
5 & Receiver Gain & 362 \\
6 & Relaxation Delay & 1.0000 \\
7 Pulse Width & 10.0000 \\
8 Acquisition Time & 6.3439 \\
9 & Spectrometer Frequency 250.13 \\
10 Spectral Width & 5165.3 \\
11 Lowest Frequency & -1038.5 \\
12 Nudeus & $1 \mathrm{H}$ \\
13 Acquired Size & 32768 \\
14 Spectral Size & 32768
\end{tabular}
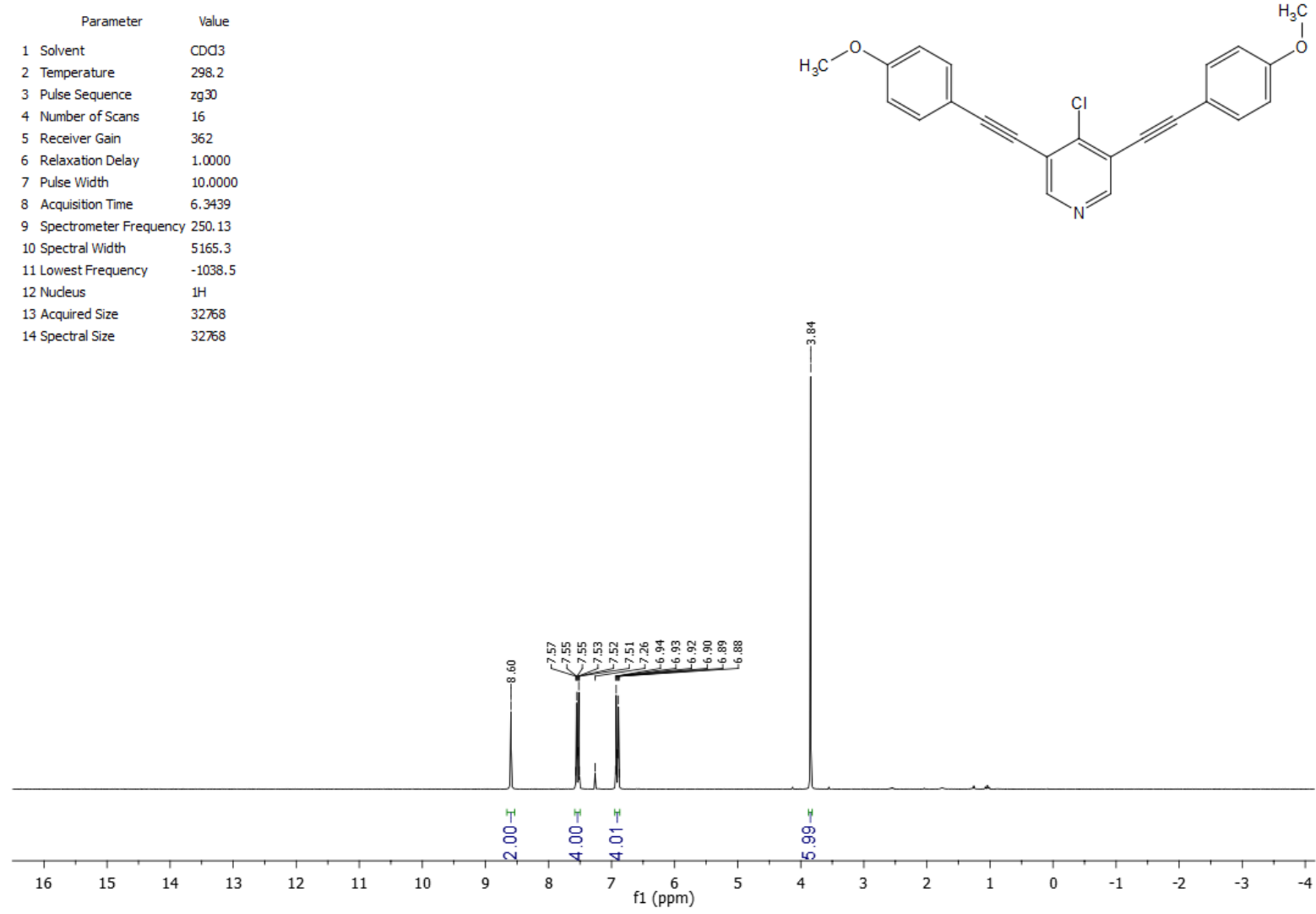

\begin{tabular}{lll}
\multicolumn{1}{c}{ Parameter } & \multicolumn{1}{c}{ Value } \\
1 Solvent & CDCl3 \\
2 2emperature & 298.0 \\
3 Pulse Sequence & $29 p 930$ \\
4 Number of Scans & 1024 \\
5 Receiver Gain & 2050 \\
6 Relaxation Delay & 2.0000 \\
7 Pulse Width & 10.0000 \\
8 Acquisition Time & 2.1846 \\
9 Spectrometer Frequency & 62.90 \\
10 Spectral Width & 15000.0 \\
11 Lowest Frequency & -1211.1 \\
12 Nucleus & $13 \mathrm{C}$ \\
13 Acquired Size & 32768 \\
14 Spectral Size & 32768
\end{tabular}

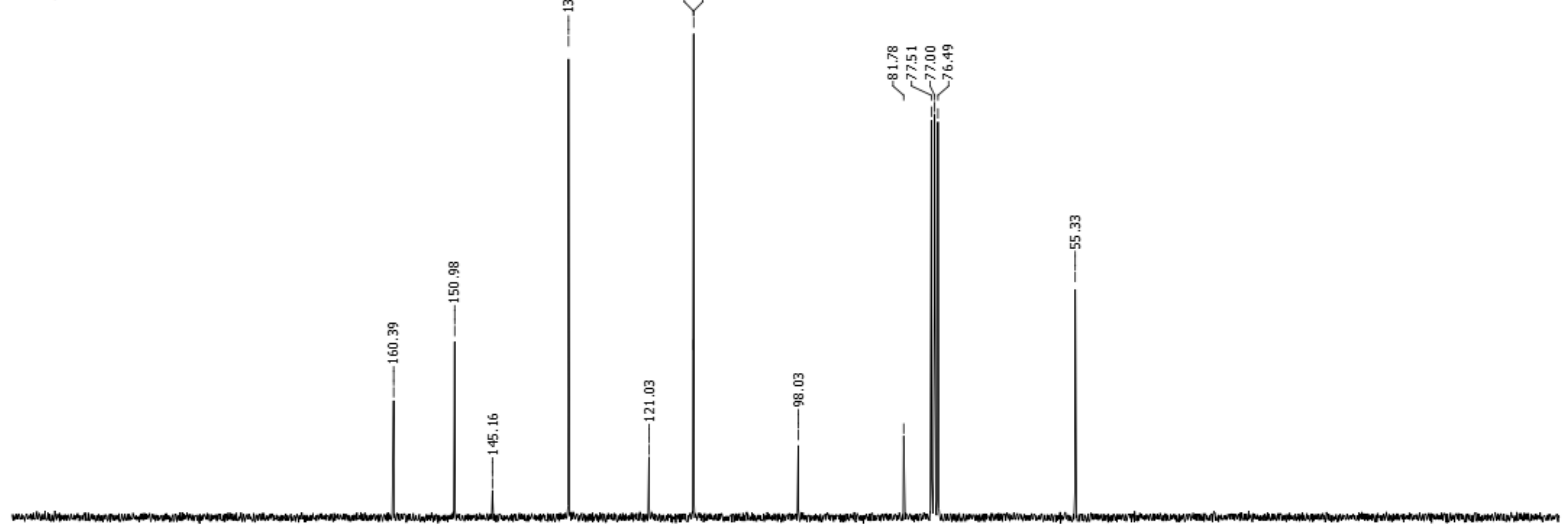

$\begin{array}{lllllllllllllllllllllllll}210 & 200 & 190 & 180 & 170 & 160 & 150 & 140 & 130 & 120 & 110 & 100 & 90 & 80 & 70 & 60 & 50 & 40 & 30 & 20 & 10 & 0 & -10\end{array}$ 
4,4'-((4-chloropyridine-3,5-diyl)bis(ethyne-2,1-diyl))bis( $N, N$-dimethylaniline) (2g)

\begin{tabular}{|c|c|c|}
\hline & Parameter & Valu \\
\hline & Solvent & $\mathrm{CDCl}$ \\
\hline & Temperature & 298. \\
\hline & Pulse Sequence & 2930 \\
\hline & Number of Scans & 16 \\
\hline & Receiver Gain & 101 \\
\hline & Relaxation Delay & 1.00 \\
\hline & Pulse Width & 8.000 \\
\hline & Acquisition Time & 3.27 \\
\hline & Spectrometer Frequency & 500 \\
\hline & Spectral Width & 1000 \\
\hline & Lowest Frequency & \\
\hline & Nucleus & $1 \mathrm{H}$ \\
\hline & Acquired Size & \\
\hline & Spectral Size & \\
\hline
\end{tabular}
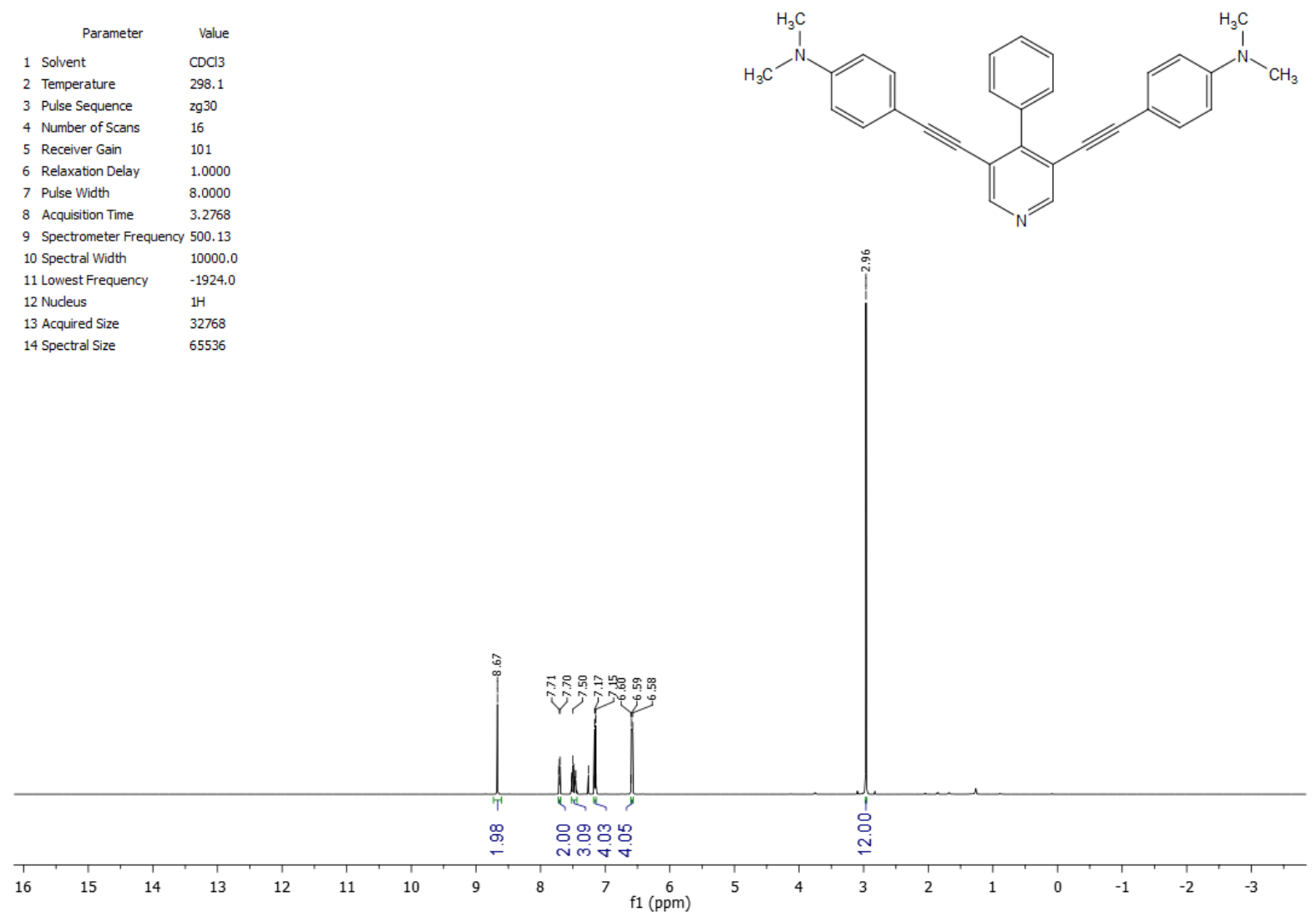

\begin{tabular}{lll}
\multicolumn{1}{c}{ Parameter } & \multicolumn{1}{c}{ Value } \\
1 Solvent & CDCl3 \\
2 & Temperature & 298.2 \\
3 3 & Pulse Sequence & 29pg 30 \\
4 Number of Scans & 2048 \\
5 Receiver Gain & 2050 \\
6 Relaxation Delay & 2.0000 \\
7 Pulse Width & 10.0000 \\
8 Acquisition Time & 2.1846 \\
9 Spectrometer Frequency 62.90 \\
10 Spectral Width & 15000.0 \\
11 Lowest Frequency & -1212.1 \\
12 Nucleus & $13 \mathrm{C}$ \\
13 Acquired Size & 32768 \\
14 Spectral Size & 32768
\end{tabular}

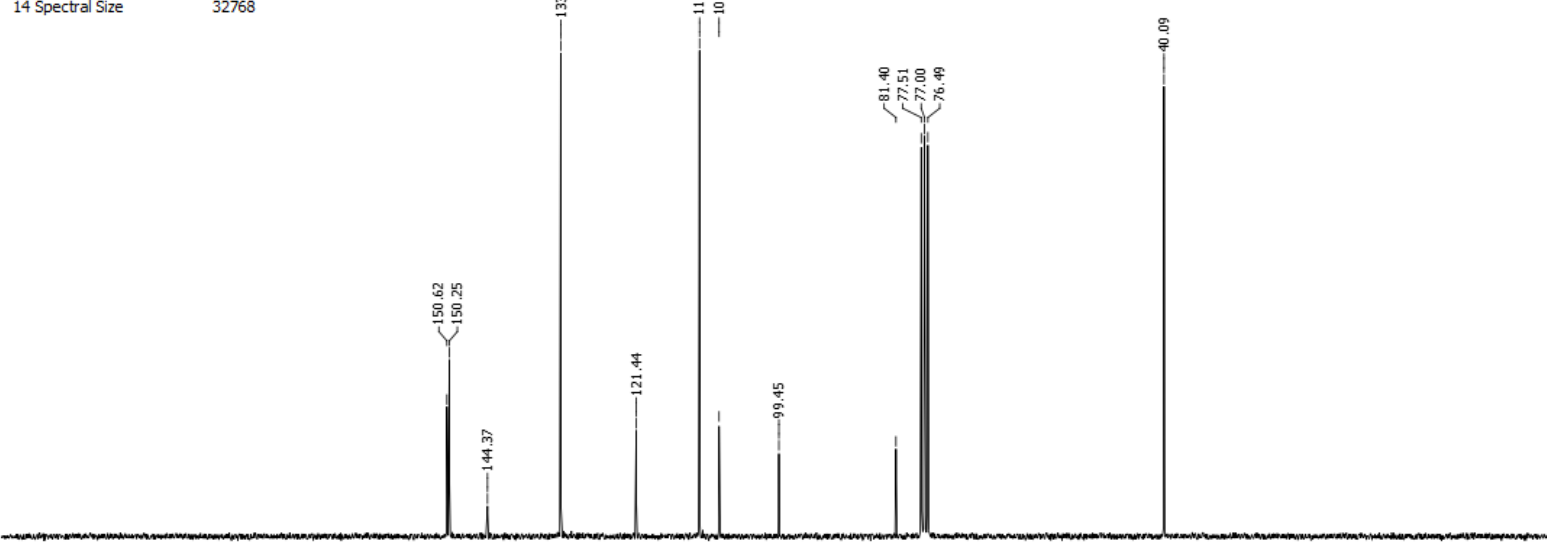

$\begin{array}{lllllllllllllllllllllll}210 & 200 & 190 & 180 & 170 & 160 & 150 & 140 & 130 & 120 & 110 & \begin{array}{c}100 \\ \mathrm{f} 1(\mathrm{ppm})\end{array} & 90 & 80 & 70 & 60 & 50 & 40 & 30 & 20 & 10 & 0 & -10\end{array}$ 
4-chloro-3,5-bis(thiophen-3-ylethynyl)pyridine (2h)

\begin{tabular}{|c|c|c|}
\hline & Parameter & Value \\
\hline & Solvent & $\mathrm{CDCl} 3$ \\
\hline & Temperature & 299.3 \\
\hline & Pulse Sequence & $2 \mathrm{~g} 30$ \\
\hline & Number of Scans & 16 \\
\hline & Receiver Gain & 812 \\
\hline & Relaxation Delay & 1.0000 \\
\hline & Pulse Width & 10.0000 \\
\hline & Acquisition Time & 6.3439 \\
\hline & Spectrometer Frequency & 250.13 \\
\hline & Spectral Width & 5165.3 \\
\hline & 1 Lowest Frequency & -1040.5 \\
\hline & 2 Nucleus & $1 \mathrm{H}$ \\
\hline & Acquired Size & 32768 \\
\hline & Spectral Size & 32768 \\
\hline
\end{tabular}
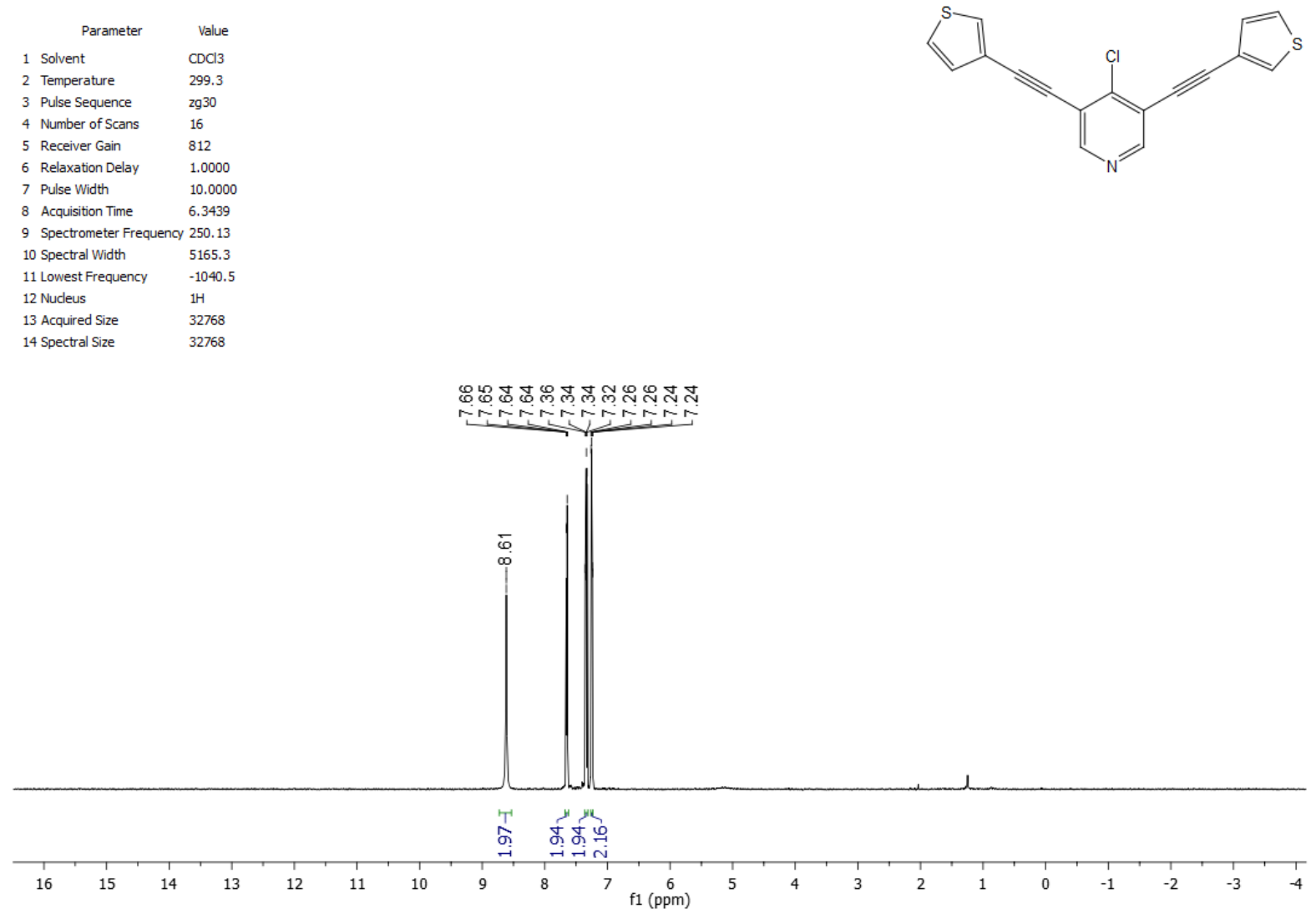

\begin{tabular}{lll}
\multicolumn{1}{c}{ Parameter } & \multicolumn{1}{c}{ Value } \\
1 Solvent & CDCl3 \\
2 2emperature & 299.9 \\
3 Pulse Sequence & 29pg30 \\
4 Number of Scans & 1024 \\
5 Receiver Gain & 2050 \\
6 Relaxation Delay & 2.0000 \\
7 Pulse Width & 10.0000 \\
8 Acquisition Time & 2.1846 \\
9 Spectrometer Frequency 62.90 \\
10 Spectral Width & 15000.0 \\
11 Lowest Frequency & -1211.3 \\
12 Nucleus & $13 \mathrm{C}$ \\
13 Acquired Size & 32768 \\
14 Spectral Size & 32768
\end{tabular}

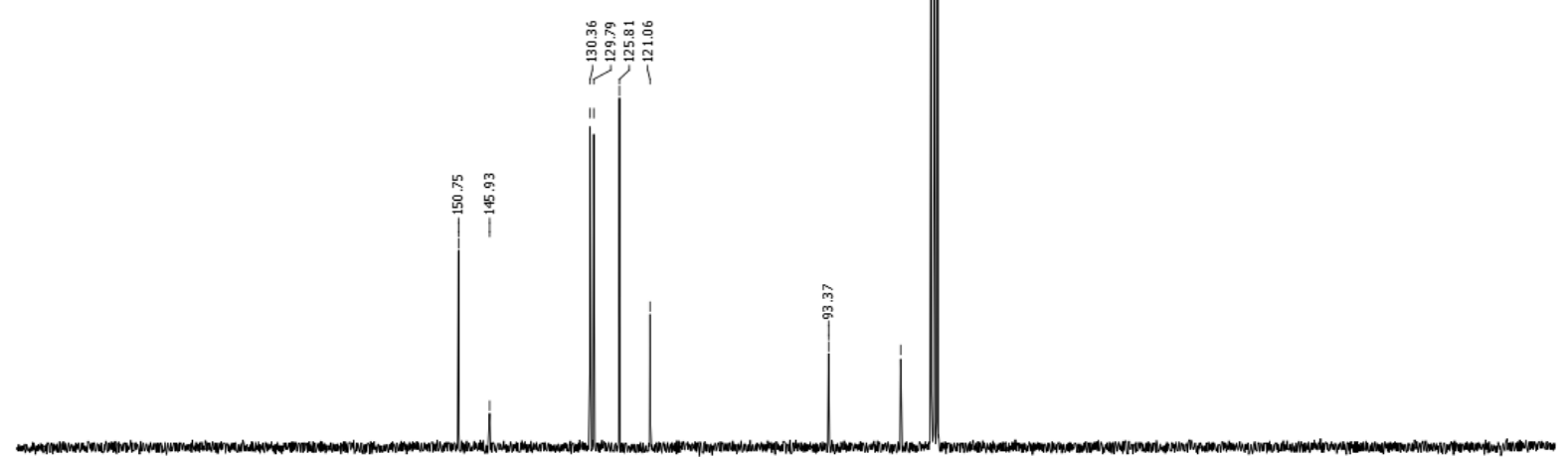

$\begin{array}{llllllllllllllllllllllllllll}210 & 200 & 190 & 180 & 170 & 160 & 150 & 140 & 130 & 120 & 110 & 100 & 90 & 80 & 70 & 60 & 50 & 40 & 30 & 20 & 10 & 0 & -10\end{array}$ 
4-phenyl-3,5-bis(phenylethynyl)pyridine (3a)

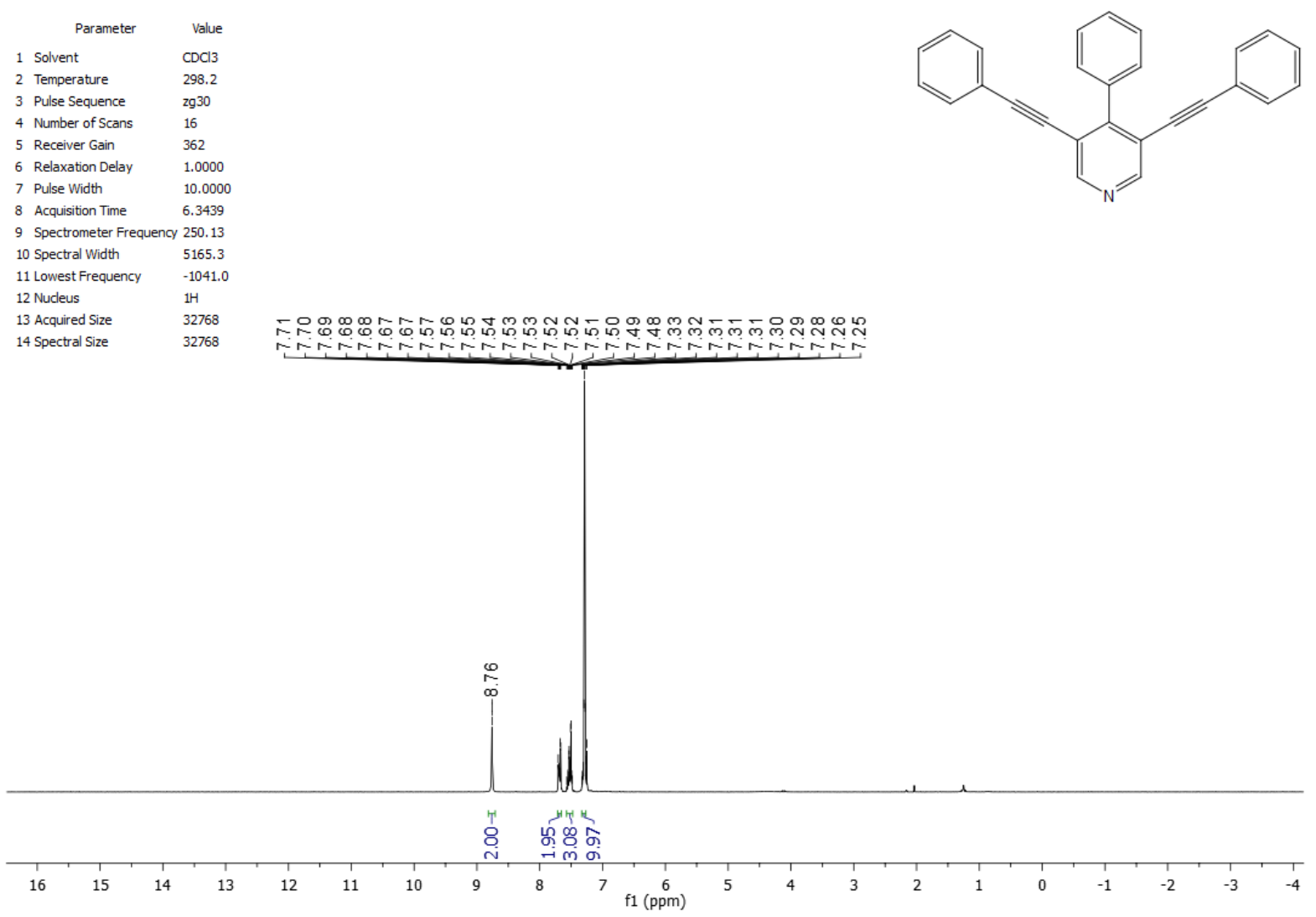

$$
\begin{array}{lll}
\multicolumn{1}{c}{\text { Parameter }} & \multicolumn{1}{c}{\text { Value }} \\
1 \text { Solvent } & \text { CDCl3 } \\
\text { 2 } & \text { Temperature } & 299.3 \\
3 \text { Pulse Sequence } & \text { 29pg } 30 \\
4 \text { Number of Scans } & 1024 \\
5 \text { Receiver Gain } & 2050 \\
6 \text { Relaxation Delay } & 2.0000 \\
7 \text { Pulse Width } & 10.0000 \\
8 \text { Acquisition Time } & 2.1846 \\
9 \text { Spectrometer Frequency } & 62.90 \\
\text { 10 } & \text { Spectral Width } & 15000.0 \\
\text { 11 Lowest Frequency } & -1212.3 \\
\text { 12 Nucleus } & 13 \mathrm{C} \\
\text { 13 Acquired Size } & 32768 \\
\text { 14 Spectral Size } & 32768
\end{array}
$$

Value
CDCl3
299.3
zgpg 30
1024
2050
2.0000
10.0000
2.1846
62.90
15000.0
-1212.3
$13 \mathrm{C}$
32768
32768

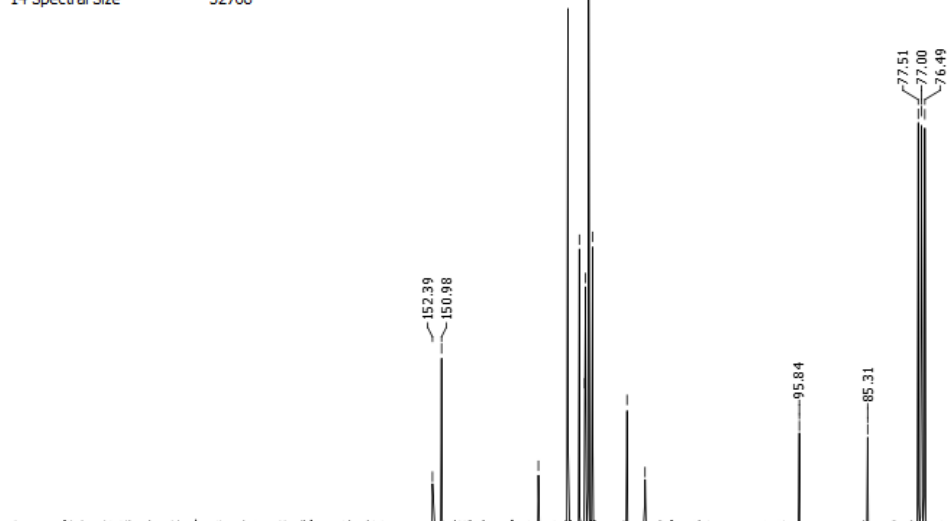

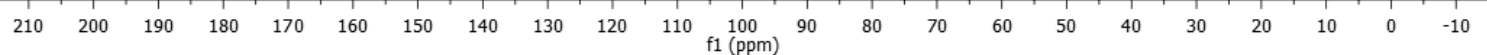


3,5-bis(phenylethynyl)-4-(p-tolyl)pyridine (3b)

\begin{tabular}{lll}
\multicolumn{1}{c}{ Parameter } & \multicolumn{1}{c}{ Value } \\
1 Solvent & CDCl3 \\
2 2emperature & 298.2 \\
3 Pulse Sequence & 2930 \\
4 Number of Scans & 16 \\
5 Receiver Gain & 101 \\
6 Relaxation Delay & 1.0000 \\
7 Pulse Width & 9.0000 \\
8 Acquisition Time & 5.2954 \\
9 Spectrometer Frequency & 300.13 \\
10 Spectral Width & 6188.1 \\
11 Lowest Frequency & -1246.9 \\
12 Nucleus & $1 H$ \\
13 Acquired Size & 32768 \\
14 Spectral Size & 32768
\end{tabular}

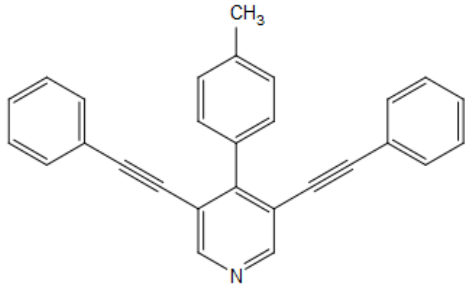

14 Spectral Size $\quad 32768$ 
4-(4-methoxyphenyl)-3,5-bis(phenylethynyl)pyridine (3c)

\begin{tabular}{lll}
\multicolumn{1}{c}{ Parameter } & \multicolumn{1}{c}{ Value } \\
1 Solvent & CDCl3 \\
2 2 & Temperature & 298.1 \\
3 Pulse Sequence & 2930 \\
4 Number of Scans & 16 \\
5 Receiver Gain & 63 \\
6 Relaxation Delay & 1.0000 \\
7 Pulse Width & 8.0000 \\
8 Acquisition Time & 3.2768 \\
9 Spectrometer Frequency 500.13 \\
10 Spectral Width & 10000.0 \\
11 Lowest Frequency & -1924.1 \\
12 Nucleus & $1 \mathrm{H}$ \\
13 Acquired Size & 32768 \\
14 Spectral Size & 65536
\end{tabular}

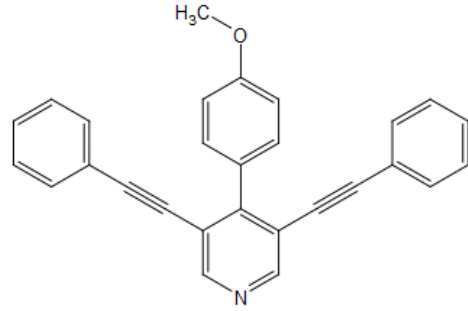

13 Acquired Size

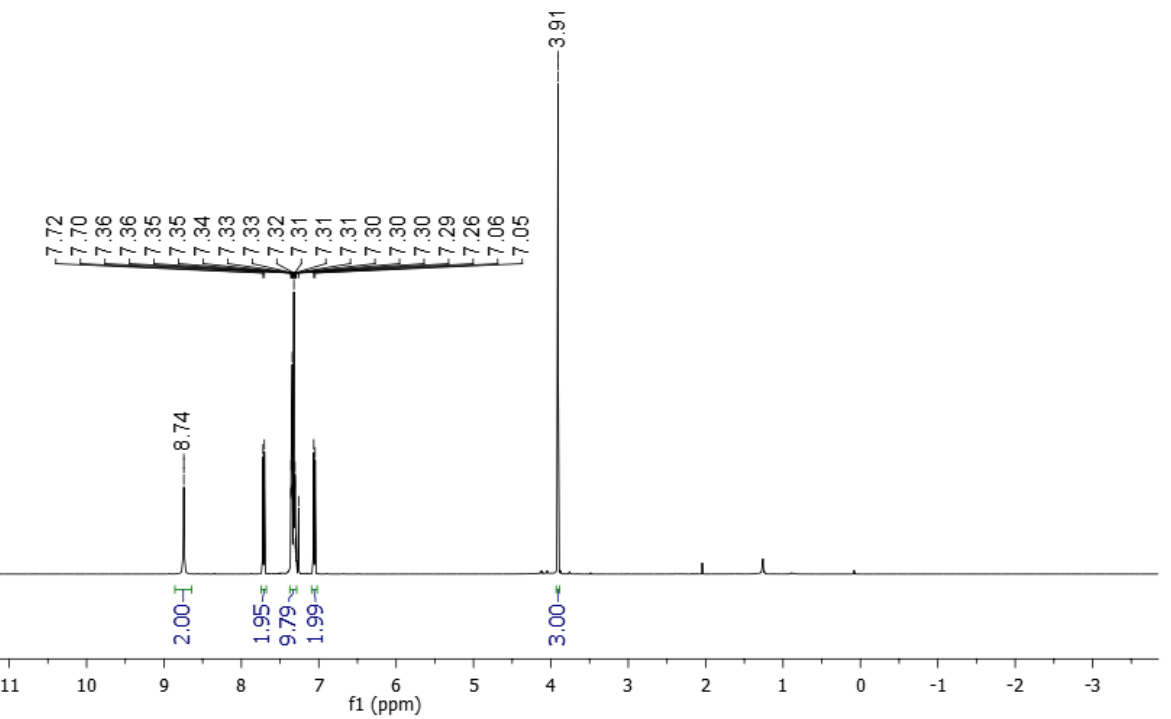

\begin{tabular}{lll} 
& \multicolumn{1}{c}{ Parameter } & \multicolumn{1}{c}{ Value } \\
1 Solvent & CDCl3 \\
2 Temperature & 298.2 \\
3 Pulse Sequence & 29pg30 \\
4 Number of Scans & 1024 \\
5 Receiver Gain & 101 \\
6 Relaxation Delay & 2.0000 \\
7 Pulse Width & 10.0000 \\
8 Acquisition Time & 1.0879 \\
9 Spectrometer Frequency 125.76 \\
10 Spectral Width & 30120.5 \\
11 Lowest Frequency & -2490.9 \\
12 Nudeus & $13 \mathrm{C}$ \\
13 Acquired Size & 32768 \\
14 Spectral Size & 32768
\end{tabular}

Value
CDCl3
28.2
0.240
102
10000
0.0000
.0879
125.76
3120.5
-2490.9
13 C
32768
32768

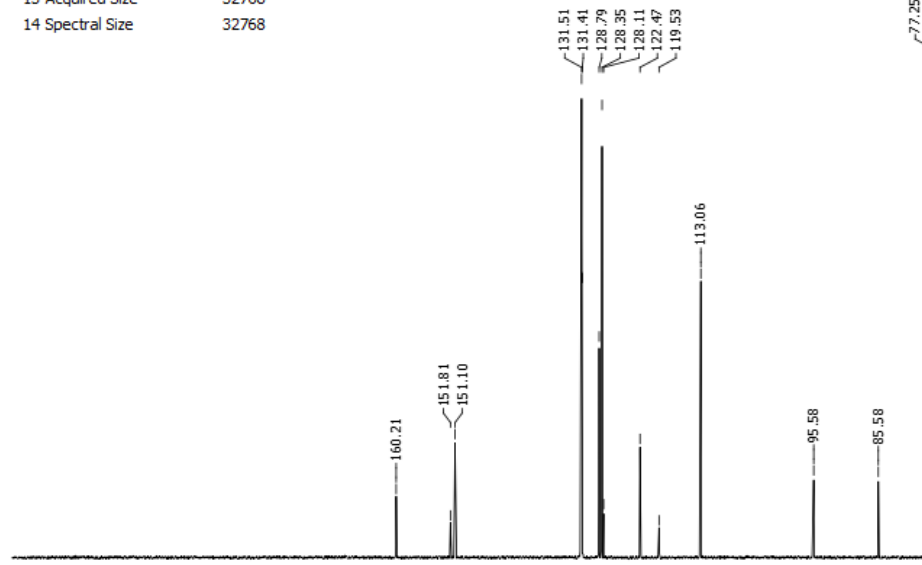

$\begin{array}{lllllllllllllllllllllllll}210 & 200 & 190 & 180 & 170 & 160 & 150 & 140 & 130 & 120 & 110 & 100 & 90 & 80 & 70 & 60 & 50 & 40 & 30 & 20 & 10 & 0 & -10\end{array}$ 
3,5-bis(phenylethynyl)-4-(4-(trifluoromethyl)phenyl)pyridine (3d)

\begin{tabular}{lll} 
& \multicolumn{1}{c}{ Parameter } & \multicolumn{1}{c}{ Value } \\
1 Solvent & CDCl3 \\
2 Temperature & 298.1 \\
3 Pulse Sequence & 2930 \\
4 Number of Scans & 64 \\
5 Receiver Gain & 63 \\
6 Relaxation Delay & 1.0000 \\
7 Pulse Width & 8.0000 \\
8 Acquisition Time & 3.2768 \\
9 Spectrometer Frequency & 500.13 \\
10 Spectral Width & 10000.0 \\
11 Lowest Frequency & -1966.6 \\
12 Nucleus & $1 \mathrm{H}$ \\
13 Acquired Size & 32768 \\
14 Spectral Size & 65536
\end{tabular}

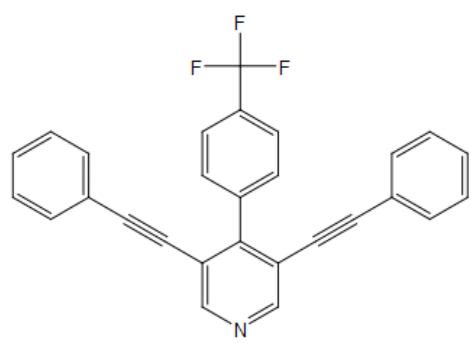

14 Spectral Size $\quad 65536$

\begin{tabular}{lll}
\multicolumn{1}{c}{ Parameter } & \multicolumn{1}{c}{ Value } \\
1 Solvent & CDCl3 \\
2 Temperature & 298.3 \\
3 & Pulse Sequence & zgig \\
4 Number of Scans & 16 \\
5 S & Receiver Gain & 101 \\
6 & Relaxation Delay & 1.0000 \\
7 Pulse Width & 12.0000 \\
8 Acquisition Time & 0.5767 \\
9 Spectrometer Frequency & 470.59 \\
10 & Spectral Width & 113636.4 \\
11 Lowest Frequency & -103877.4 \\
12 Nucleus & $19 F$ \\
13 Acquired Size & 65536 \\
14 Spectral Size & 65536
\end{tabular}




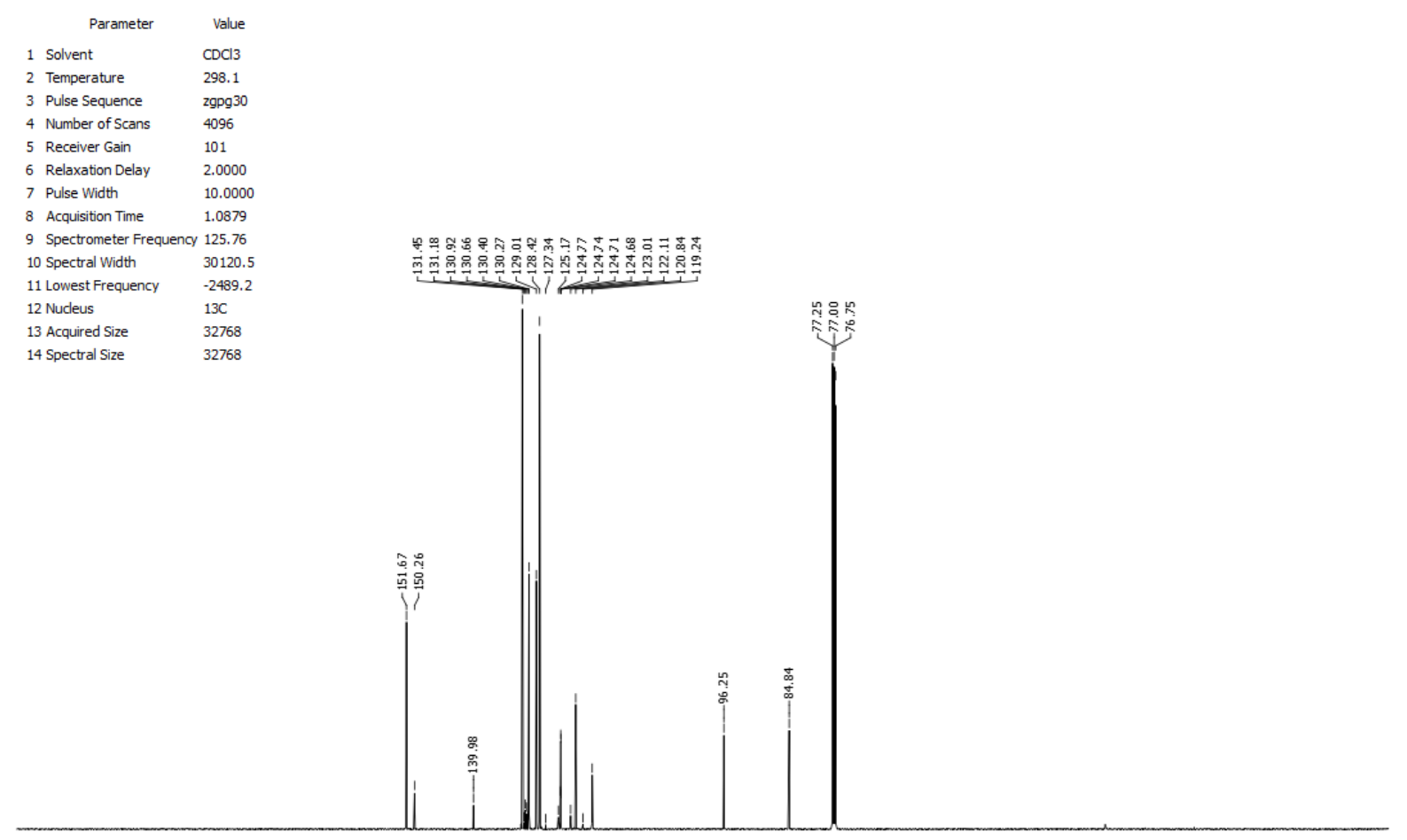

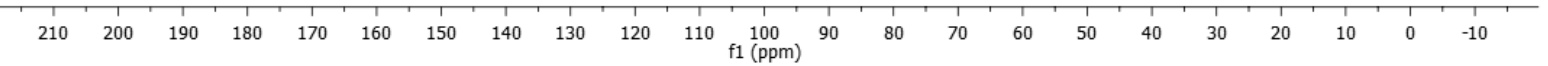


4-phenyl-3,5-bis( $p$-tolylethynyl)pyridine (Be)

\begin{tabular}{lll}
\multicolumn{1}{c}{ Parameter } & \multicolumn{1}{c}{ Value } \\
1 Solvent & $\mathrm{CDCl} 3$ \\
2 Temperature & 298.2 \\
3 Pulse Sequence & $2 \mathrm{z30}$ \\
4 Number of Scans & 16 \\
5 Receiver Gain & 645 \\
6 Relaxation Delay & 1.0000 \\
7 Pulse Width & 10.0000 \\
8 Acquisition Time & 6.3439 \\
9 Spectrometer Frequency & 250.13 \\
10 Spectral Width & 5165.3 \\
11 Lowest Frequency & -1038.5 \\
12 Nucleus & $1 \mathrm{H}$ \\
13 Acquired Size & 32768 \\
14 Spectral Size & 32768
\end{tabular}
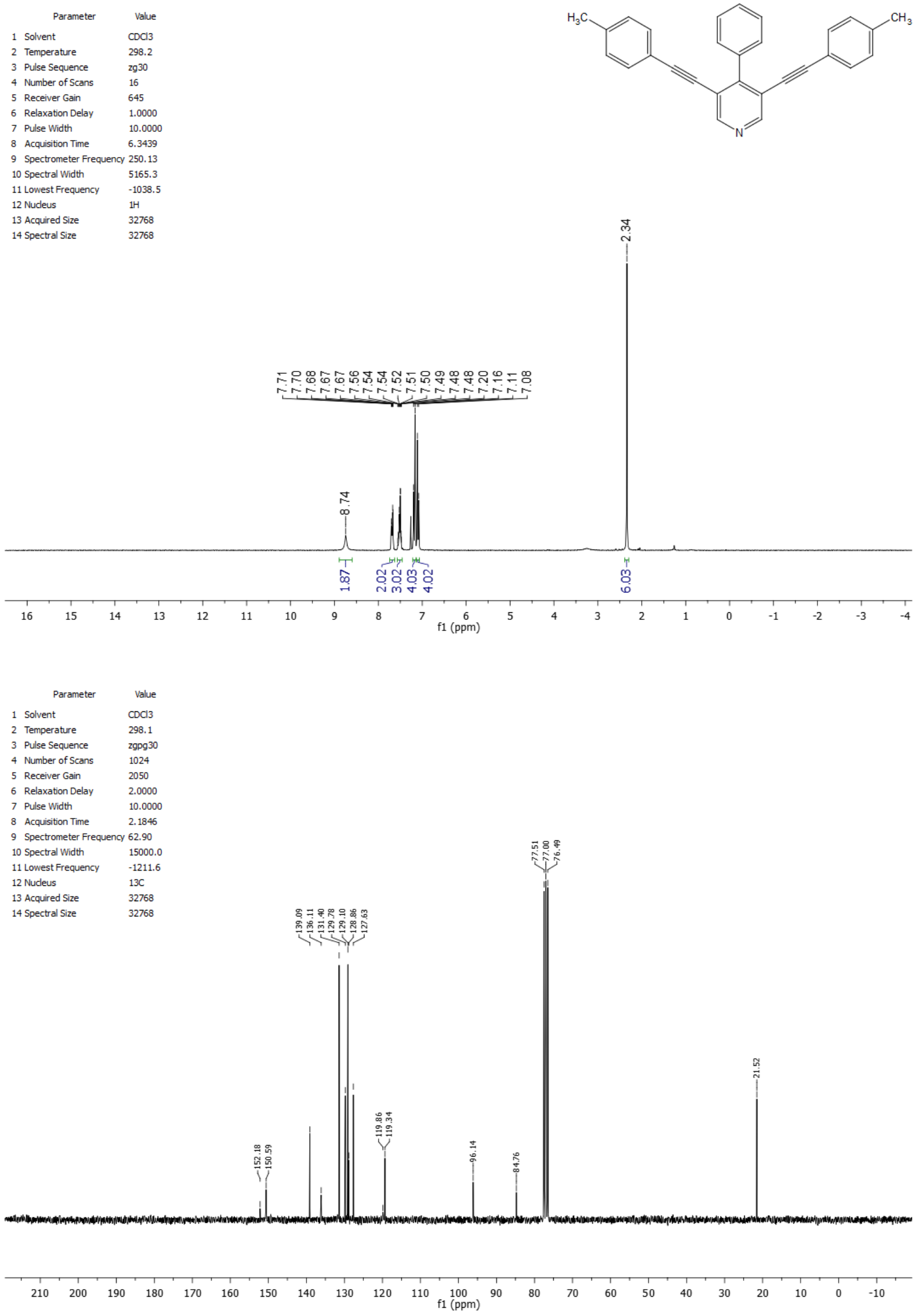

$\mathrm{S} 60$ 
4-(p-tolyl)-3,5-bis( $p$-tolylethynyl)pyridine (3f)

\begin{tabular}{lll}
\multicolumn{1}{c}{ Parameter } & \multicolumn{1}{c}{ Value } \\
1 Solvent & CDCl3 \\
2 & Temperature & 298.3 \\
3 & Pulse Sequence & 2930 \\
4 Number of Scans & 16 \\
5 5 & Receiver Gain & 575 \\
6 6elaxation Delay & 1.0000 \\
7 Pulse Width & 10.0000 \\
8 & Acquisition Time & 6.3439 \\
9 Spectrometer Frequency 250.13 \\
10 Spectral Width & 5165.3 \\
11 Lowest Frequency & -1038.5 \\
12 Nucleus & $1 \mathrm{H}$ \\
13 Acquired Size & 32768 \\
14 Spectral Size & 32768
\end{tabular}
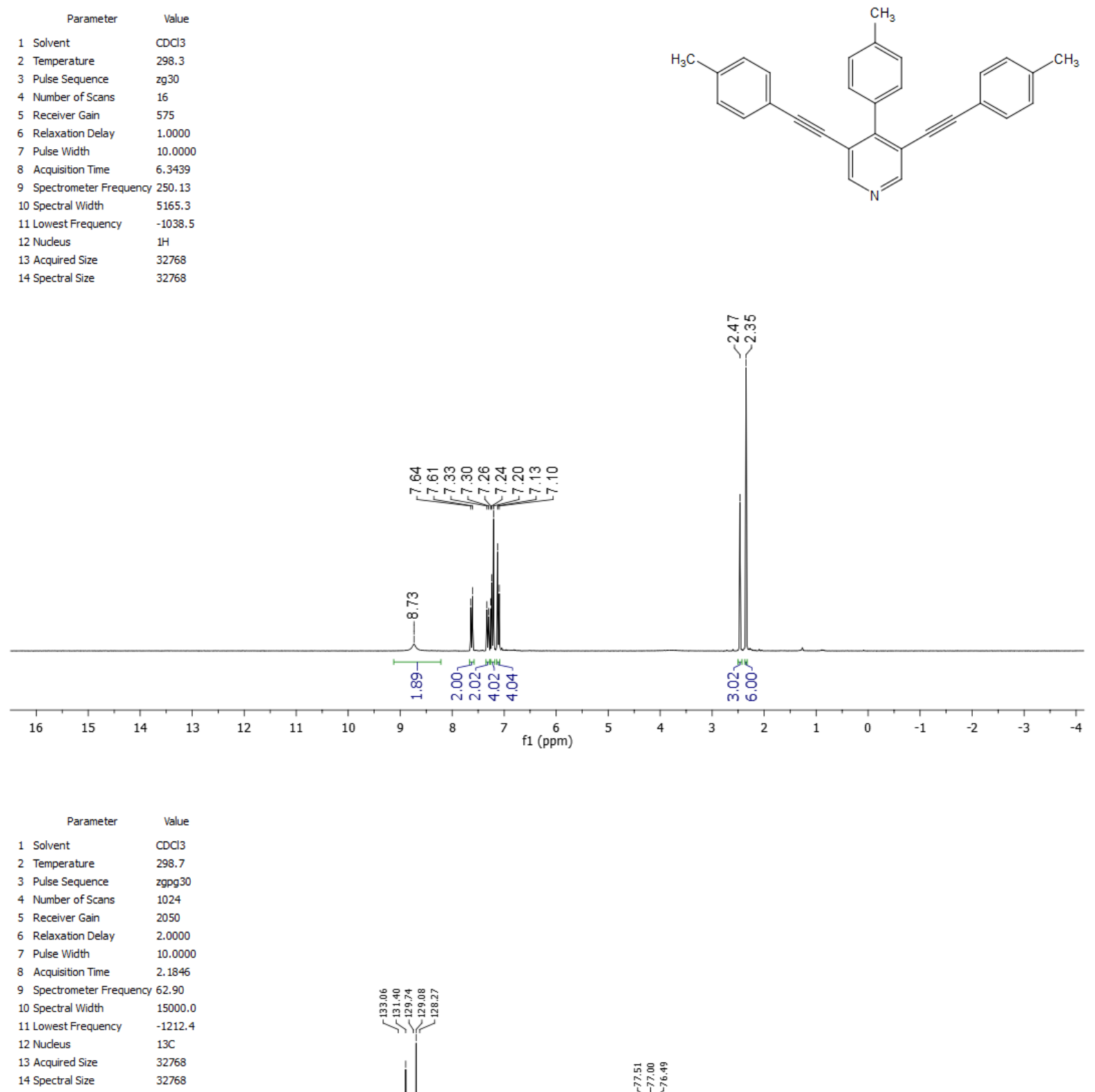

Value
CDCl3
298.7
9 pg 30
1024
2050
.0000
0.0000
2.1846
2.90
5000.0
1212.4
$13 \mathrm{C}$
32768
32768

$$
14 \text { Spectral Size } \quad 3276
$$

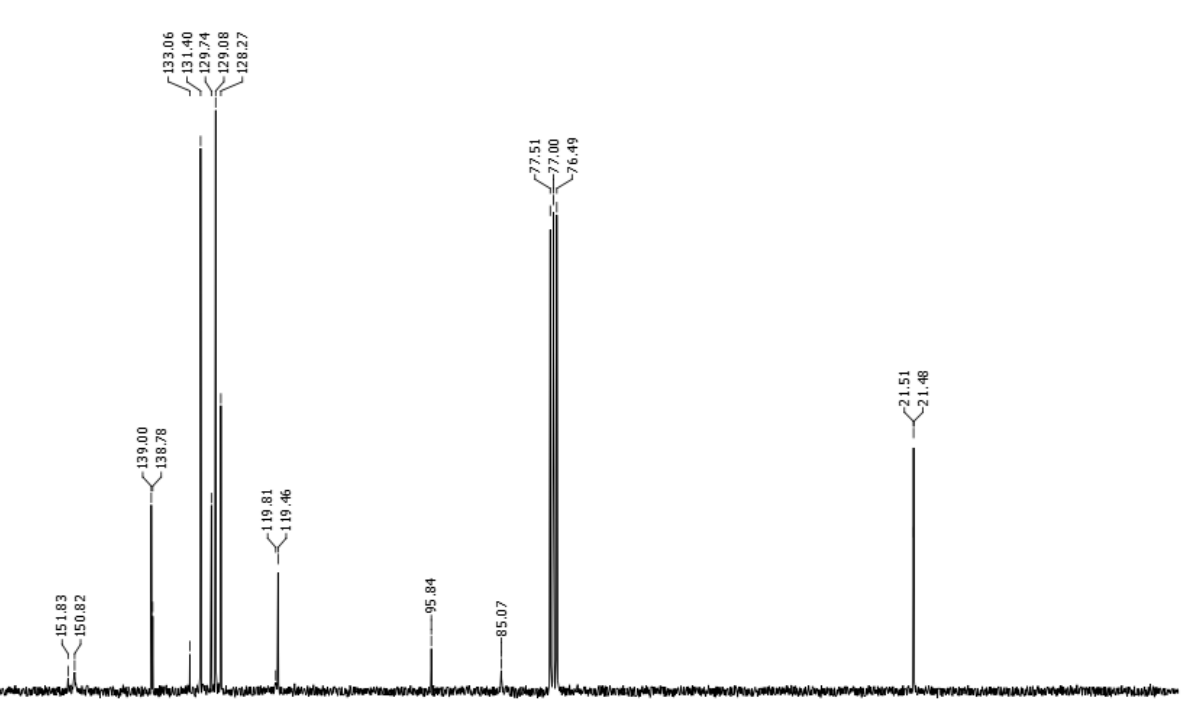

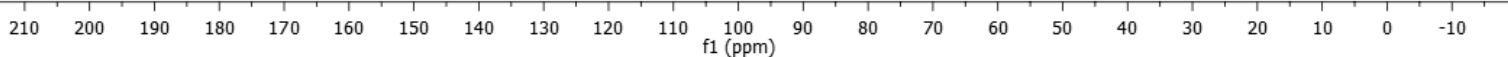


4-(4-methoxyphenyl)-3,5-bis(p-tolylethynyl)pyridine (3g)

\begin{tabular}{lll}
\multicolumn{1}{c}{ Parameter } & \multicolumn{1}{c}{ Value } \\
1 Solvent & CDCl3 \\
2 Temperature & 298.2 \\
3 Pulse Sequence & 2930 \\
4 Number of Scans & 16 \\
5 Receiver Gain & 63 \\
6 Relaxation Delay & 1.0000 \\
7 Pulse Width & 8.0000 \\
8 Acquisition Time & 3.2768 \\
9 Spectrometer Frequency 500.13 \\
10 Spectral Width & 10000.0 \\
11 Lowest Frequency & -1924.0 \\
12 Nucleus & $1 H$ \\
13 Acquired Size & 32768 \\
14 Spectral Size & 65536
\end{tabular}

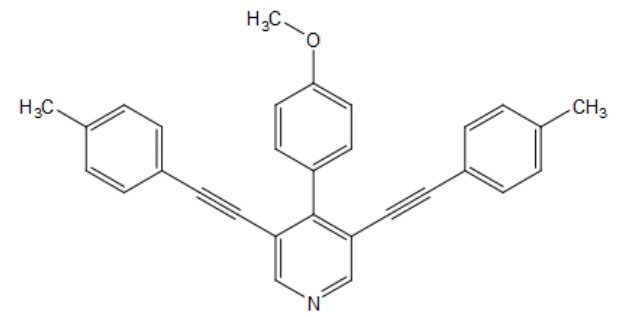

14 Spectral Size

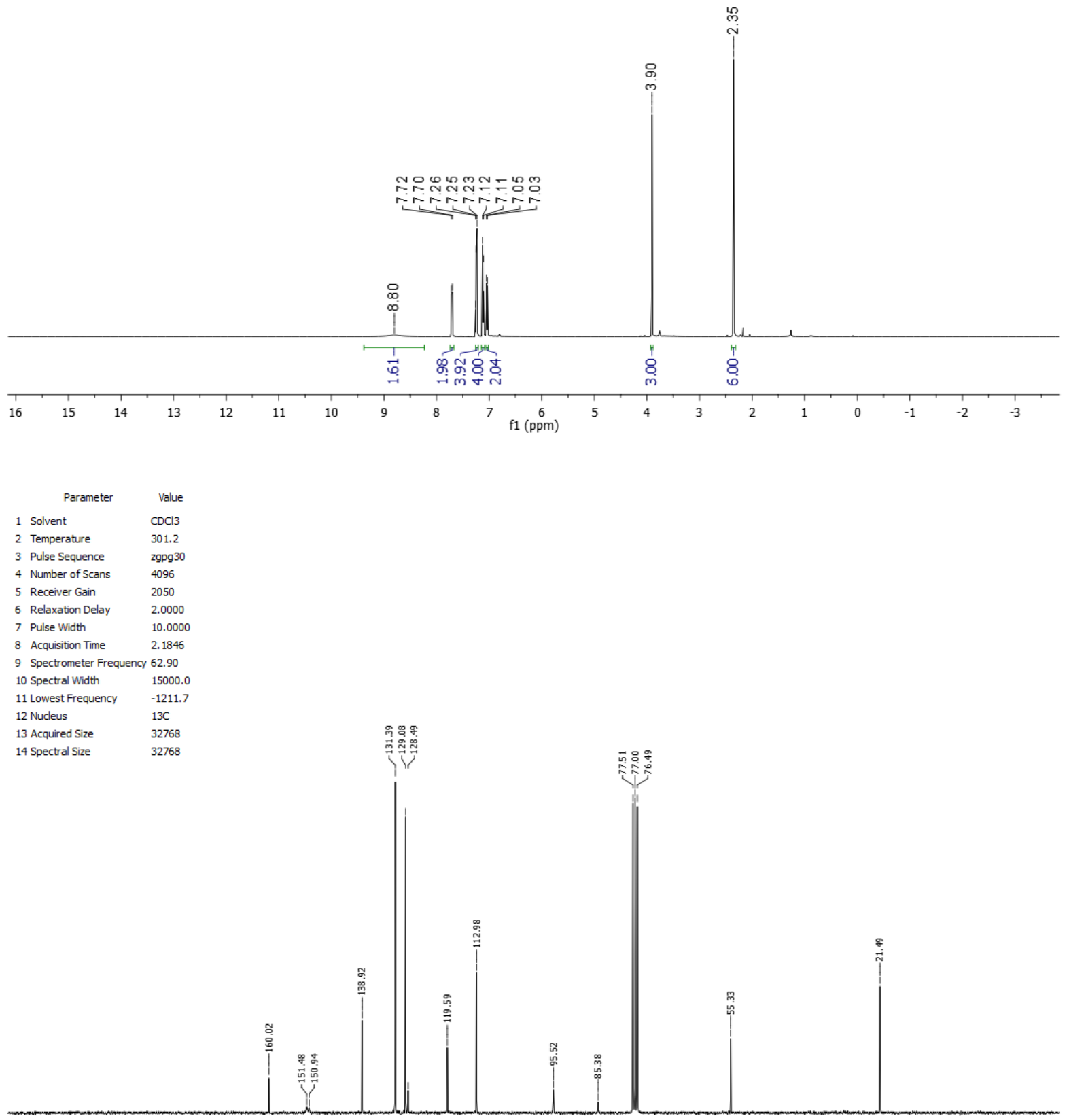

$\begin{array}{llllllllllllllllllllllll}210 & 200 & 190 & 180 & 170 & 160 & 150 & 140 & 130 & 120 & 110 & 100 & 90 & 80 & 70 & 60 & 50 & 40 & 30 & 20 & 10 & 0 & -10\end{array}$ 
3,5-bis(p-tolylethynyl)-4-(4-(trifluoromethyl)phenyl)pyridine (3h)

\begin{tabular}{lll}
\multicolumn{1}{c}{ Parameter } & \multicolumn{1}{c}{ Value } \\
1 Solvent & CDCl3 \\
2 Temperature & 298.2 \\
3 Pulse Sequence & 2930 \\
4 Number of Scans & 16 \\
5 Receiver Gain & 63 \\
6 Relaxation Delay & 1.0000 \\
7 Pulse Width & 8.0000 \\
8 Acquisition Time & 3.2768 \\
9 Spectrometer Frequency 500.13 \\
10 Spectral Width & 10000.0 \\
11 Lowest Frequency & -1924.3 \\
12 Nucleus & $1 \mathrm{H}$ \\
13 Acquired Size & 32768 \\
14 Spectral Size & 65536
\end{tabular}
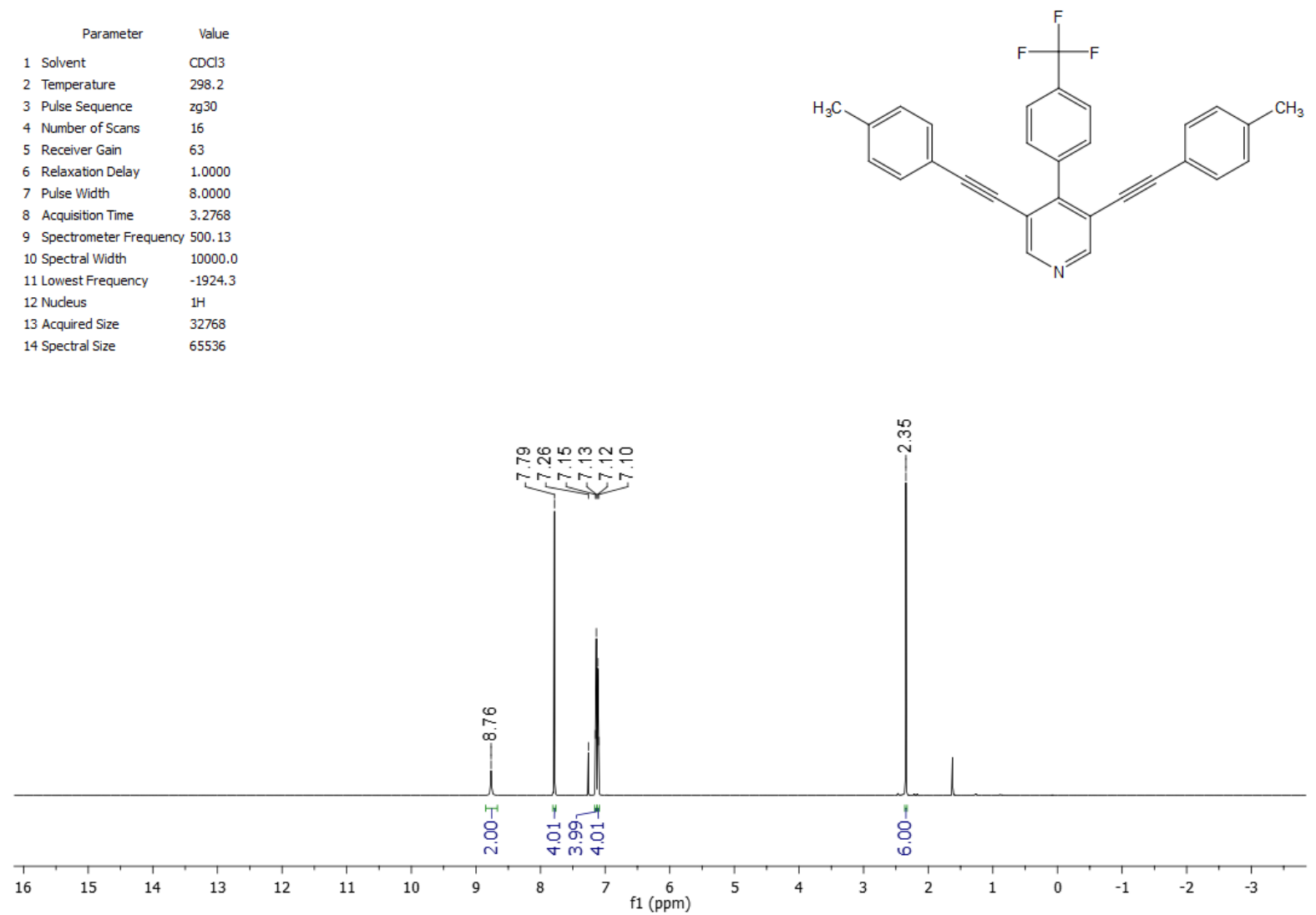

\begin{tabular}{lll}
\multicolumn{1}{c}{ Parameter } & \multicolumn{1}{c}{ Value } \\
1 Solvent & CDCl3 \\
2 2 & Temperature & 298.3 \\
3 Pulse Sequence & 2gig \\
4 Number of Scans & 16 \\
5 Receiver Gain & 101 \\
6 Relaxation Delay & 1.0000 \\
7 Pulse Width & 12.0000 \\
8 Acquisition Time & 0.5767 \\
9 Spectrometer Frequency & 470.59 \\
10 Spectral Width & 113636.4 \\
11 Lowest Frequency & -103877.4 \\
12 Nucleus & $19 F$ \\
13 Acquired Size & 65536 \\
14 Spectral Size & 65536
\end{tabular}

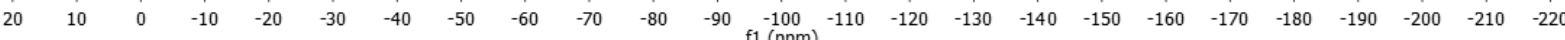




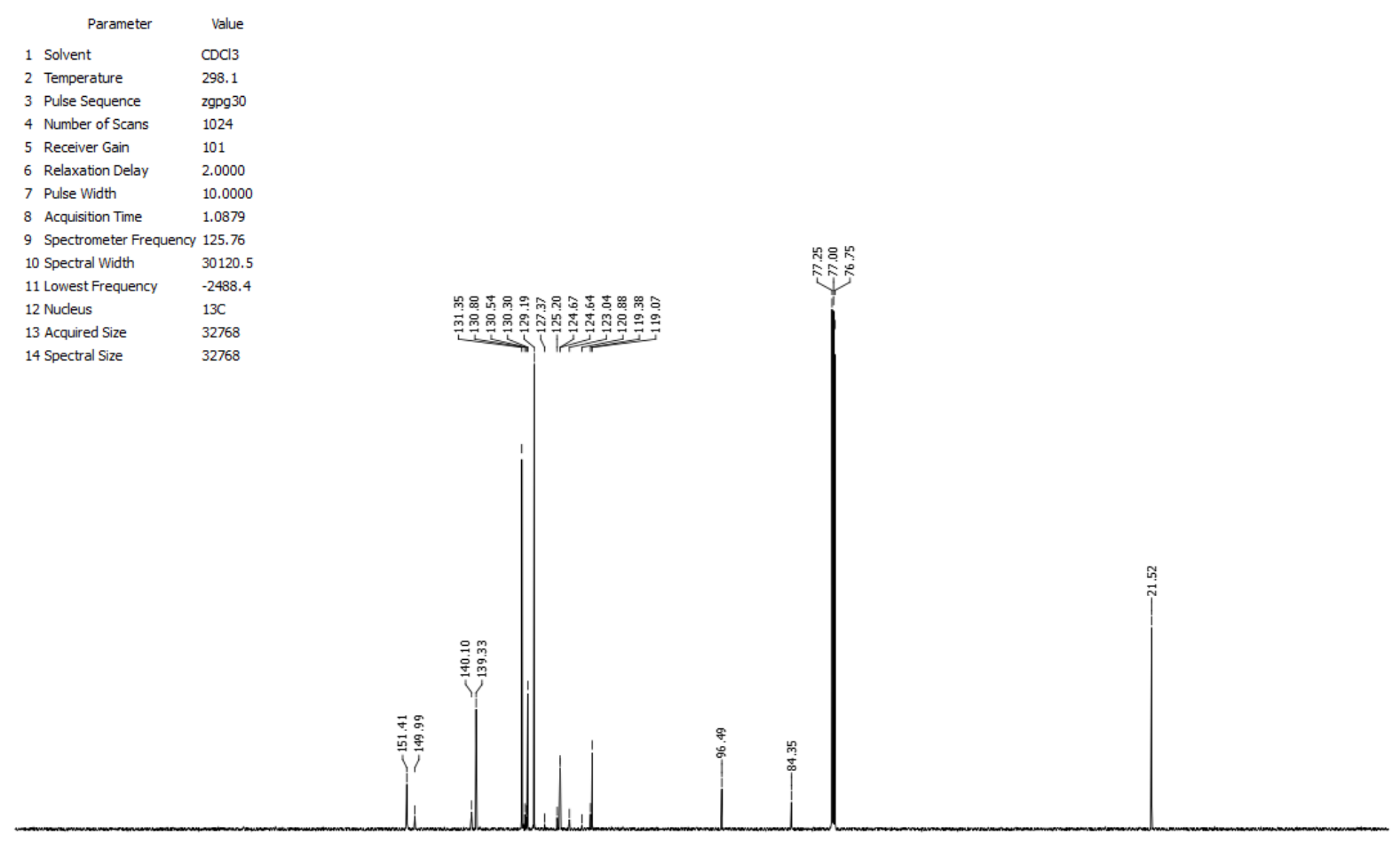

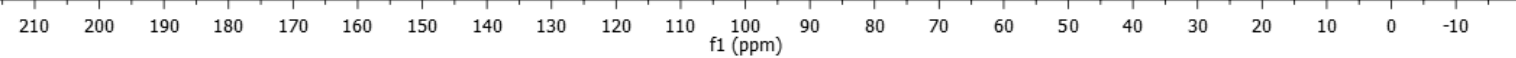


4-phenyl-3,5-bis( $m$-tolylethynyl)pyridine (3i)

\begin{tabular}{|c|c|c|}
\hline & Parameter & Valu \\
\hline & Solvent & $\mathrm{CDCl}_{3}$ \\
\hline & Temperature & 298.2 \\
\hline & Pulse Sequence & 2930 \\
\hline & Number of Scans & 16 \\
\hline & Receiver Gain & 724 \\
\hline & Relaxation Delay & 1.00 \\
\hline & Pulse Width & 10.0 \\
\hline & Acquisition Time & 6.3439 \\
\hline & Spectrometer Frequenc, & 250 \\
\hline & Spectral Width & 5165 \\
\hline & Lowest Frequency & -103 \\
\hline & Nudeus & $1 \mathrm{H}$ \\
\hline & Acquired Size & 327 \\
\hline & Spectral Size & 3276 \\
\hline
\end{tabular}
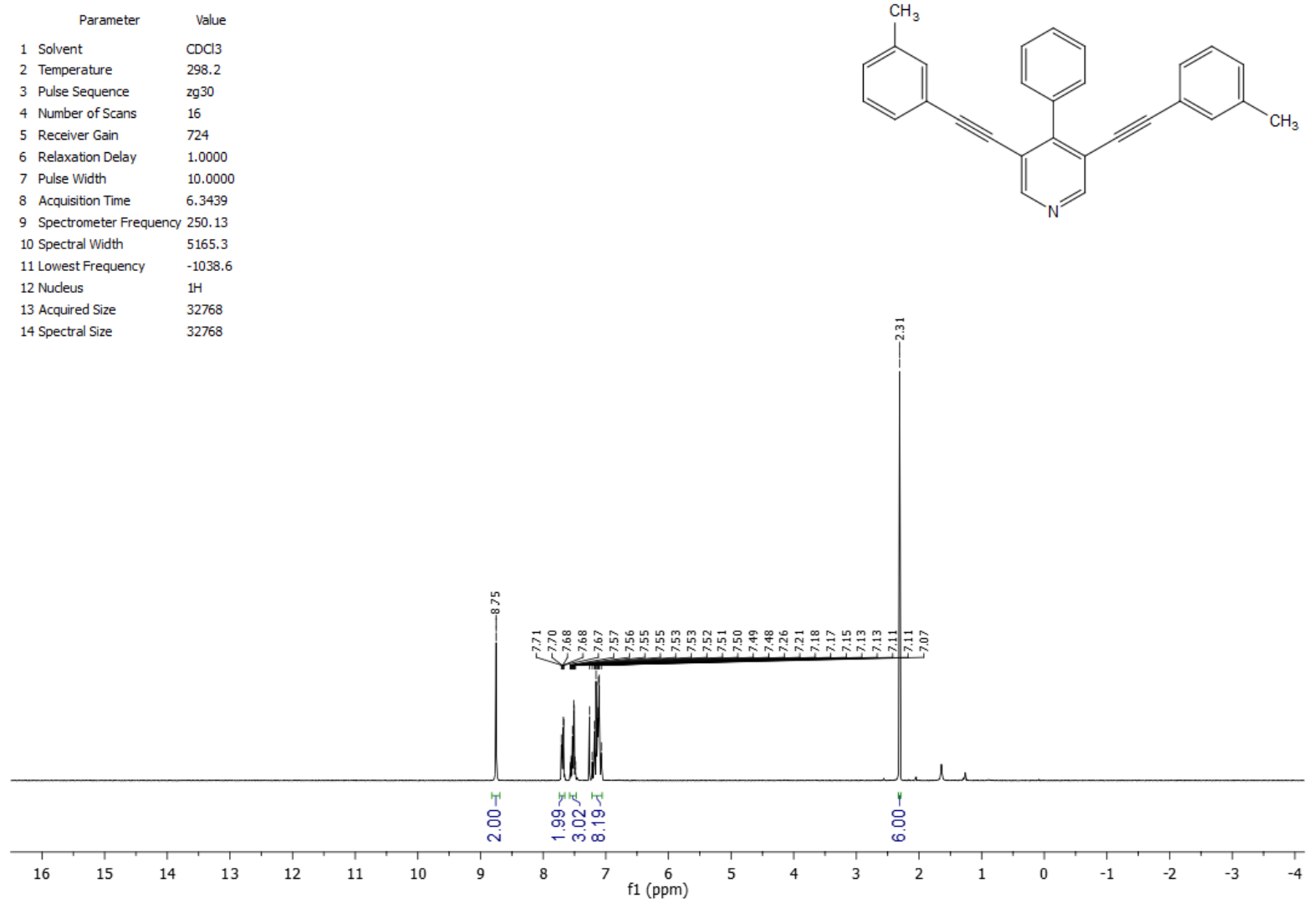

\begin{tabular}{|c|c|c|}
\hline & Parameter & Value \\
\hline & Solvent & $\mathrm{CDCl} 3$ \\
\hline & Temperature & 298.2 \\
\hline & Pulse Sequence & zgpg 30 \\
\hline & Number of Scans & 1024 \\
\hline & Receiver Gain & 2050 \\
\hline & Relaxation Delay & 2.0000 \\
\hline & Pulse Width & 10.0000 \\
\hline & Acquisition Time & 2. 1846 \\
\hline & Spectrometer Frequency & 62.90 \\
\hline & Spectral Width & 15000.0 \\
\hline & Lowest Frequency & -1211.3 \\
\hline & Nucleus & $13 \mathrm{C}$ \\
\hline & Acquired Size & 32768 \\
\hline & pectral Size & 32768 \\
\hline
\end{tabular}

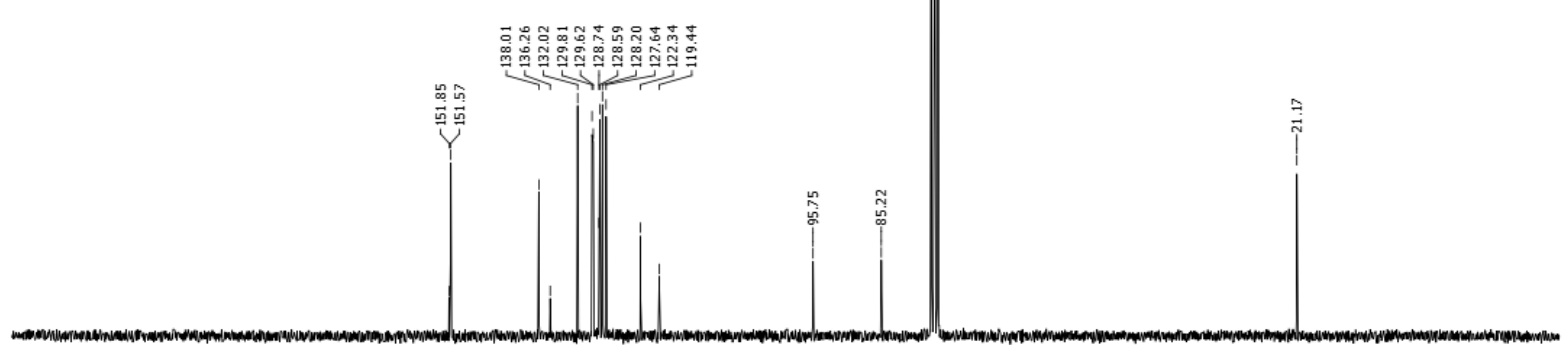

$\begin{array}{lllllllllllllllllllllll}210 & 200 & 190 & 180 & 170 & 160 & 150 & 140 & 130 & 120 & 110 & \begin{array}{c}100 \\ \mathrm{f} 1(\mathrm{ppm})\end{array} & 90 & 80 & 70 & 60 & 50 & 40 & 30 & 20 & 10 & 0 & -10\end{array}$ 
4-(p-tolyl)-3,5-bis( $m$-tolylethynyl)pyridine (3j)

\begin{tabular}{lll}
\multicolumn{1}{c}{ Parameter } & \multicolumn{1}{c}{ Value } \\
1 Solvent & CDCl3 \\
2 & Temperature & 298.2 \\
3 & Pulse Sequence & 2930 \\
4 & Number of Scans & 16 \\
5 S & Receiver Gain & 63 \\
6 6elaxation Delay & 1.0000 \\
7 Pulse Width & 8.0000 \\
8 & Acquisition Time & 3.2768 \\
9 & Spectrometer Frequency 500.13 \\
10 & Spectral Width & 10000.0 \\
11 Lowest Frequency & -1924.2 \\
12 Nucleus & $1 \mathrm{H}$ \\
13 Acquired Size & 32768 \\
14 Spectral Size & 65536
\end{tabular}
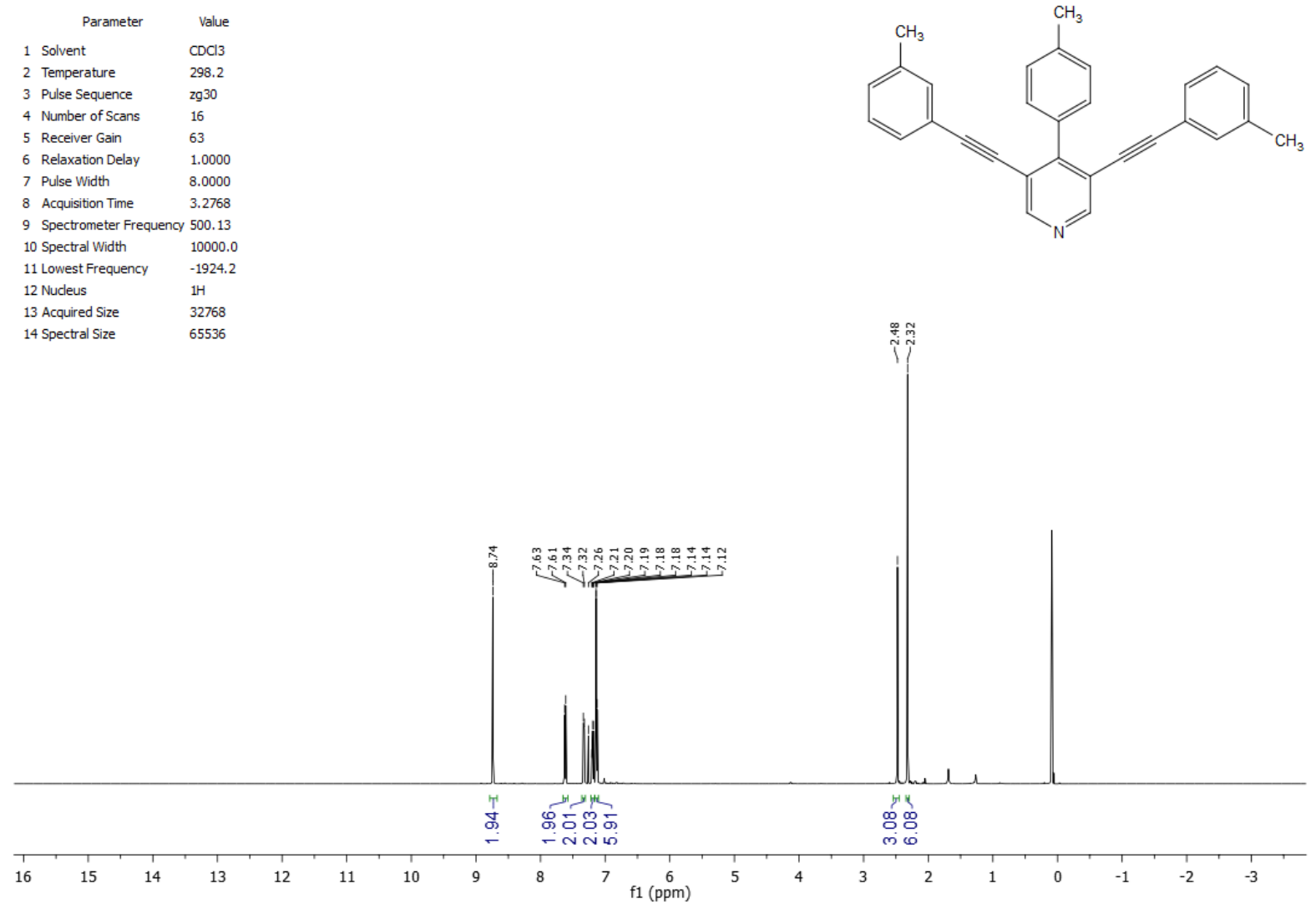

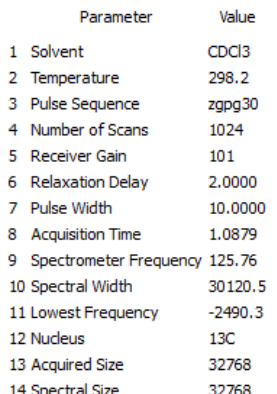

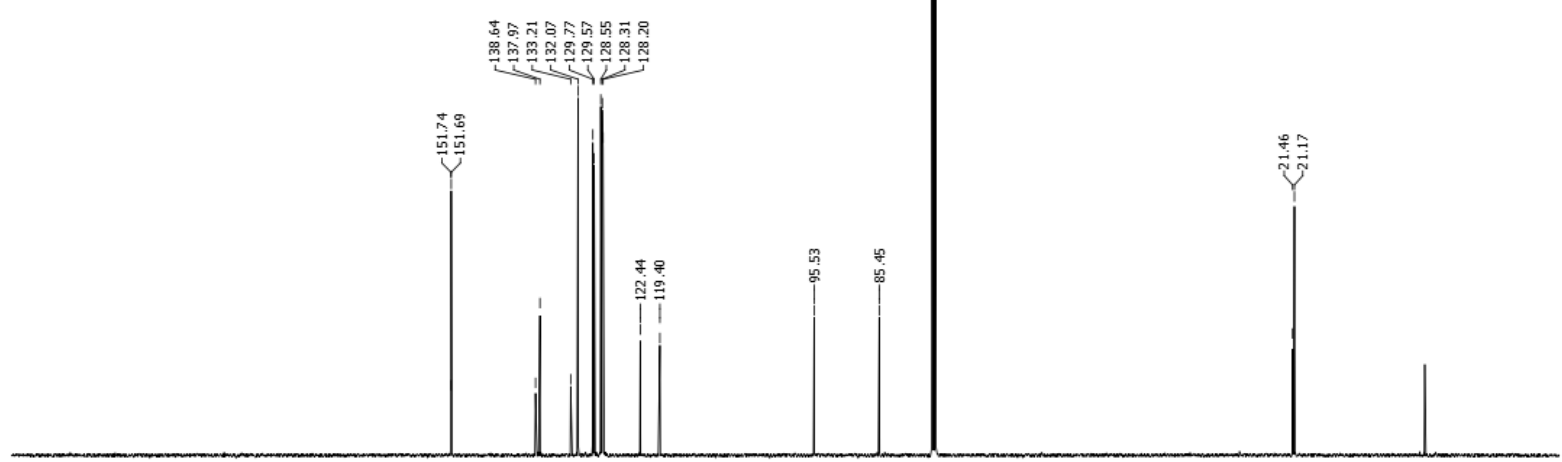

$\begin{array}{lllllllllllllllllllllll}210 & 200 & 190 & 180 & 170 & 160 & 150 & 140 & 130 & 120 & 110 & 100 & 90 & 80 & 70 & 60 & 50 & 40 & 30 & 20 & 10 & 0 & -10\end{array}$ 


$$
\begin{array}{lll} 
& \multicolumn{1}{c}{\text { Parameter }} & \multicolumn{1}{c}{\text { Value }} \\
1 & \text { Solvent } & \text { CDCl3 } \\
2 & \text { Temperature } & 298.2 \\
3 \text { Pulse Sequence } & 2930 \\
4 \text { N } & \text { Number of Scans } & 16 \\
5 & \text { Receiver Gain } & 362 \\
6 & \text { Relaxation Delay } & 1.0000 \\
7 \text { Pulse Width } & 10.0000 \\
8 \text { Acquisition Time } & 6.3439 \\
9 & \text { Spectrometer Frequency } 250.13 \\
10 & \text { Spectral Width } & 5165.3 \\
11 \text { Lowest Frequency } & -1038.5 \\
\text { 12 Nucleus } & 1 H \\
\text { 13 Acquired Size } & 32768 \\
14 \text { Spectral Size } & 32768
\end{array}
$$
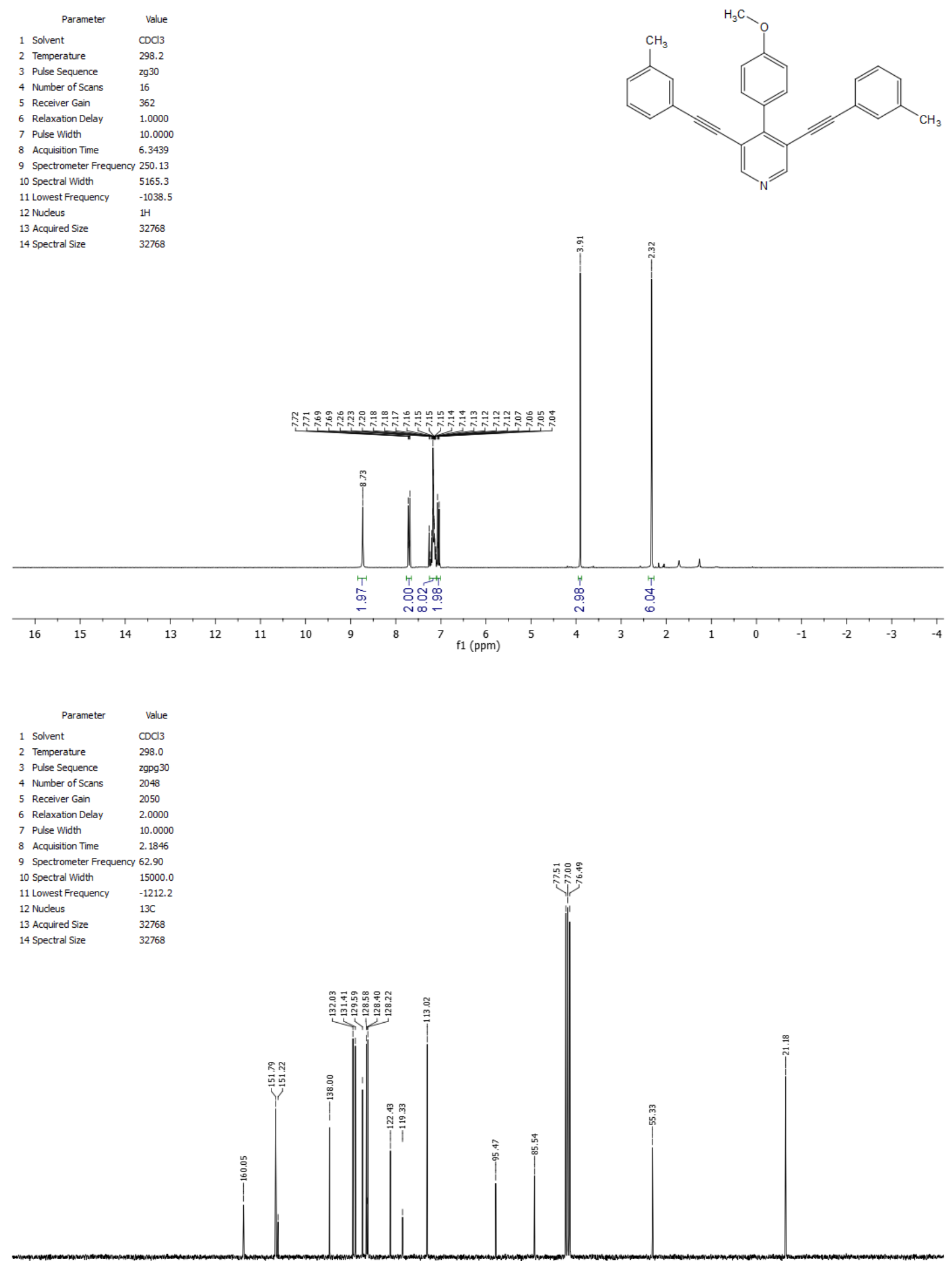

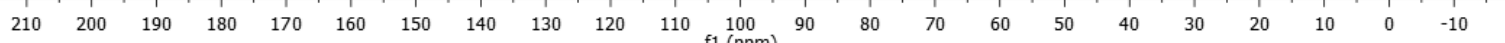


3,5-bis((4-fluorophenyl)ethynyl)-4-phenylpyridine (3I)

\begin{tabular}{lll}
\multicolumn{1}{c}{ Parameter } & \multicolumn{1}{c}{ Value } \\
1 Solvent & CDCl3 \\
2 Temperature & 298.1 \\
3 Pulse Sequence & 2930 \\
4 Number of Scans & 16 \\
5 Receiver Gain & 63 \\
6 Relaxation Delay & 1.0000 \\
7 Pulse Width & 8.0000 \\
8 Acquisition Time & 3.2768 \\
9 Spectrometer Frequency 500.13 \\
10 Spectral Width & 10000.0 \\
11 Lowest Frequency & -1923.9 \\
12 Nucleus & $1 \mathrm{H}$ \\
13 Acquired Size & 32768 \\
14 Spectral Size & 65536
\end{tabular}
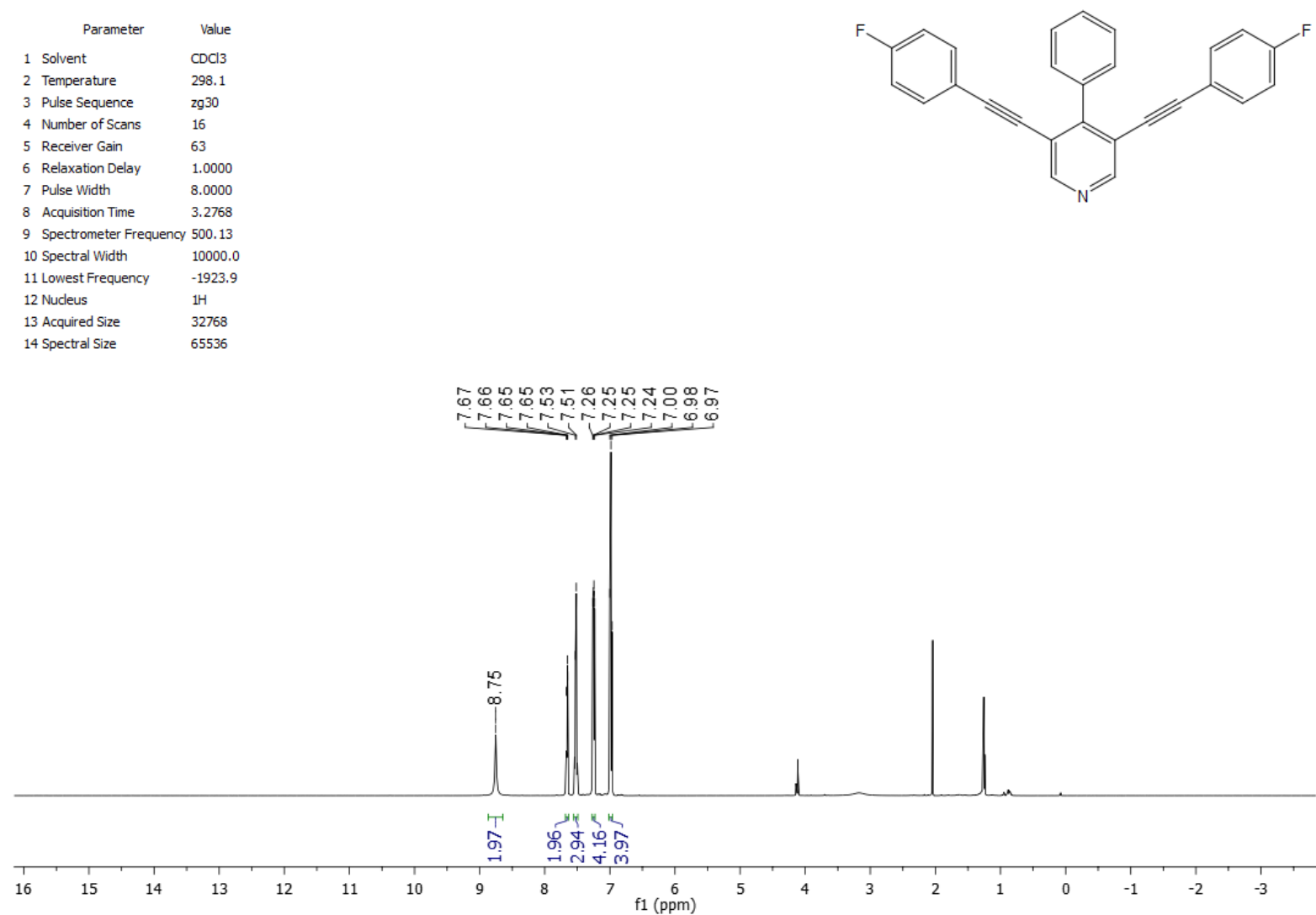

\begin{tabular}{lll}
\multicolumn{1}{c}{ Parameter } & \multicolumn{1}{c}{ Value } \\
1 Solvent & CDCl3 \\
2 Temperature & 298.3 \\
3 & Pulse Sequence & zgig \\
4 Number of Scans & 16 \\
5 5eceiver Gain & 101 \\
6 Relaxation Delay & 1.0000 \\
7 Pulse Width & 12.0000 \\
8 Acquisition Time & 0.5767 \\
9 & Spectrometer Frequency & 470.59 \\
10 & Spectral Width & 113636.4 \\
11 Lowest Frequency & -103877.4 \\
12 Nucleus & $19 F$ \\
13 Acquired Size & 65536 \\
14 Spectral Size & 65536
\end{tabular}

14 Spectral Size $\quad 65536$

$\begin{array}{lllllllllllllllllllllllllll} & 20 & 10 & 0 & -10 & -20 & -30 & -40 & -50 & -60 & -70 & -80 & -90 & -100 & -110 & -120 & -130 & -140 & -150 & -160 & -170 & -180 & -190 & -200 & -210 & -220\end{array}$ 


$$
\begin{array}{lll} 
& \multicolumn{1}{c}{\text { Parameter }} & \multicolumn{1}{c}{\text { Value }} \\
1 \text { Solvent } & \text { CDCl3 } \\
2 \text { Temperature } & 298.1 \\
3 \text { Pulse Sequence } & \text { 29pg } 30 \\
4 \text { Number of Scans } & 1024 \\
5 \text { Receiver Gain } & 101 \\
6 \text { Relaxation Delay } & 2.0000 \\
7 \text { Pulse Width } & 10.0000 \\
8 \text { Acquisition Time } & 1.0879 \\
9 \text { Spectrometer Frequency } & 125.76 \\
10 \text { Spectral Width } & 30120.5 \\
\text { 11 Lowest Frequency } & -2489.4 \\
\text { 12 Nucleus } & 13 \mathrm{C} \\
\text { 13 Acquired Size } & 32768 \\
14 \text { Spectral Size } & 32768
\end{array}
$$

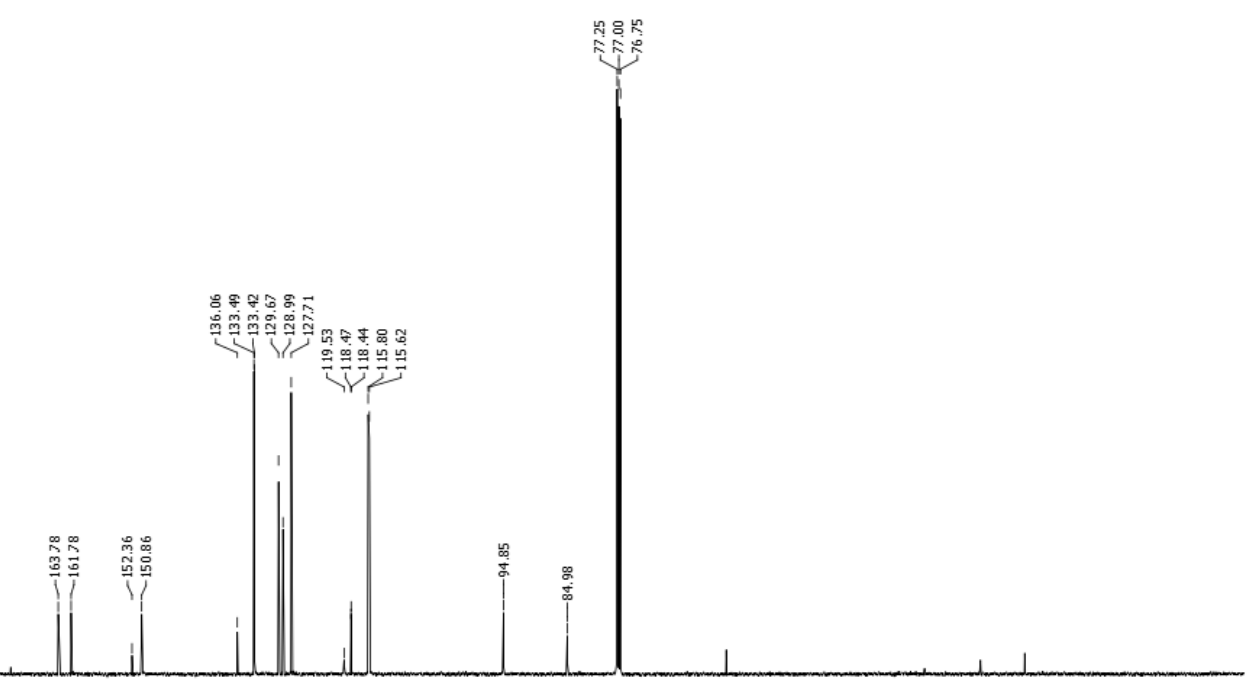

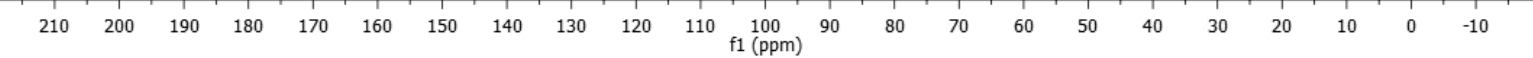


3,5-bis((4-fluorophenyl)ethynyl)-4-(p-tolyl)pyridine (3m)

\begin{tabular}{|c|c|c|}
\hline & Parameter & Value \\
\hline & Solvent & $\mathrm{CDCl} 3$ \\
\hline & Temperature & 298.2 \\
\hline & Pulse Sequence & 2930 \\
\hline & Number of Scans & 16 \\
\hline & Receiver Gain & 63 \\
\hline & Relaxation Delay & 1.0000 \\
\hline & Pulse Width & 8.0000 \\
\hline & Acquisition Time & 3.2768 \\
\hline & Spectrometer Frequency & 500.13 \\
\hline & Spectral Width & 10000.0 \\
\hline & Lowest Frequency & -1923.8 \\
\hline & Nudleus & $1 \mathrm{H}$ \\
\hline & Acquired Size & 32768 \\
\hline & Spectral Size & 65536 \\
\hline
\end{tabular}
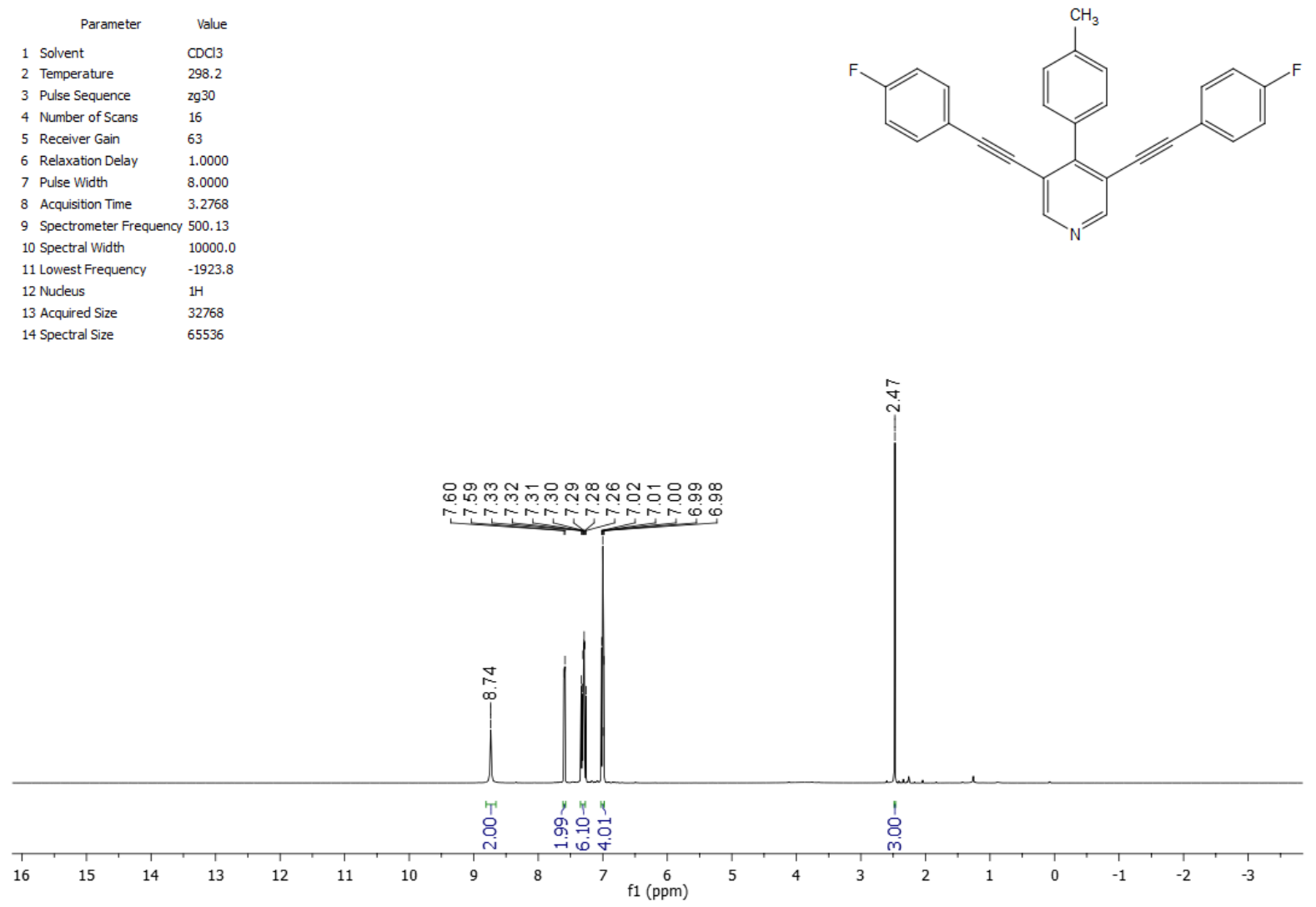

\begin{tabular}{lll}
\multicolumn{1}{c}{ Parameter } & \multicolumn{1}{c}{ Value } \\
1 Solvent & CDCl3 \\
2 2emperature & 298.3 \\
3 Pulse Sequence & 29ig \\
4 Number of Scans & 16 \\
5 Receiver Gain & 101 \\
6 Relaxation Delay & 1.0000 \\
7 Pulse Width & 12.0000 \\
8 Acquisition Time & 0.5767 \\
9 Spectrometer Frequency & 470.59 \\
10 Spectral Width & 113636.4 \\
11 Lowest Frequency & -103877.4 \\
12 Nucleus & $19 F$ \\
13 Acquired Size & 65536 \\
14 Spectral Size & 65536
\end{tabular}

$\begin{array}{lllllllllllllllllllllllll}20 & 10 & 0 & -10 & -20 & -30 & -40 & -50 & -60 & -70 & -80 & -90 & -100 & -110 & -120 & -130 & -140 & -150 & -160 & -170 & -180 & -190 & -200 & -210 & -220\end{array}$ 


$$
\begin{aligned}
& \text { Parameter Value } \\
& 1 \text { Solvent } \mathrm{CDCl} 3 \\
& 2 \text { Temperature } 299.1 \\
& 3 \text { Pulse Sequence } 2 \text { gpg } 30 \\
& 4 \text { Number of Scans } 1024 \\
& 5 \text { Receiver Gain } 2050 \\
& 6 \text { Relaxation Delay } \quad 2.0000 \\
& 7 \text { Pulse Width } \quad 10.000 \\
& 8 \text { Acquistion Time } 2.1846 \\
& 9 \text { Spectrometer Frequency } 62.90 \\
& 10 \text { Spectral Width } \quad 15000 . \\
& 11 \text { Lowest Frequency } \quad-1211.1 \\
& 12 \text { Nucleus } \quad 13 \mathrm{C} \\
& 13 \text { Acquired Size } \quad 32768 \\
& 14 \text { Spectral Size } \quad 32768
\end{aligned}
$$

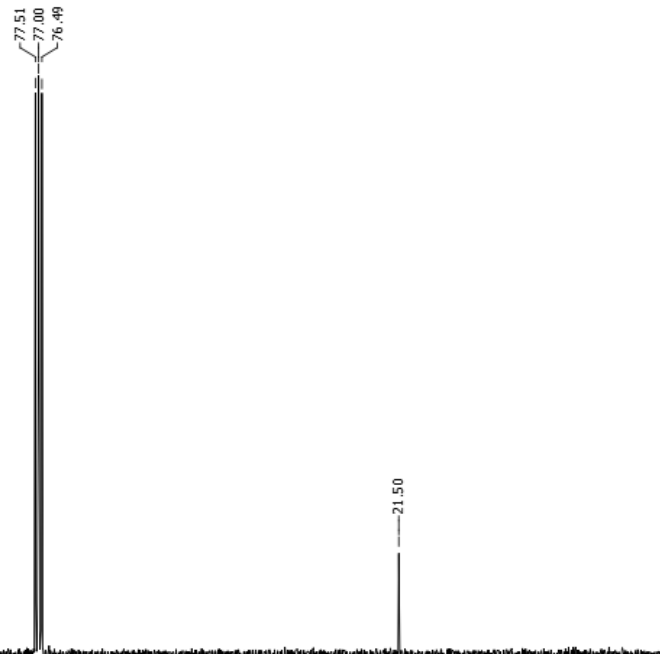

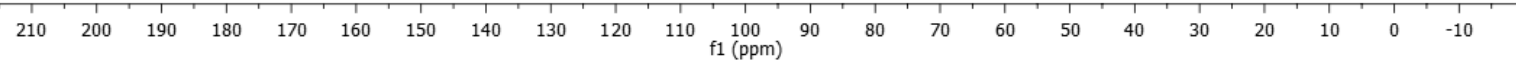




\section{3,5-bis((4-fluorophenyl)ethynyl)-4-(4-methoxyphenyl)pyridine (3n)}

\begin{tabular}{lll} 
& \multicolumn{1}{c}{ Parameter } & \multicolumn{1}{c}{ Value } \\
1 Solvent & CDCl3 \\
2 Temperature & 298.1 \\
3 Pulse Sequence & 2930 \\
4 Number of Scans & 16 \\
5 Receiver Gain & 63 \\
6 Relaxation Delay & 1.0000 \\
7 Pulse Width & 8.0000 \\
8 Acquisition Time & 3.2768 \\
9 Spectrometer Frequency 500.13 \\
10 Spectral Width & 10000.0 \\
11 Lowest Frequency & -1924.1 \\
12 Nucleus & $1 \mathrm{H}$ \\
13 Acquired Size & 32768 \\
14 Spectral Size & 65536
\end{tabular}
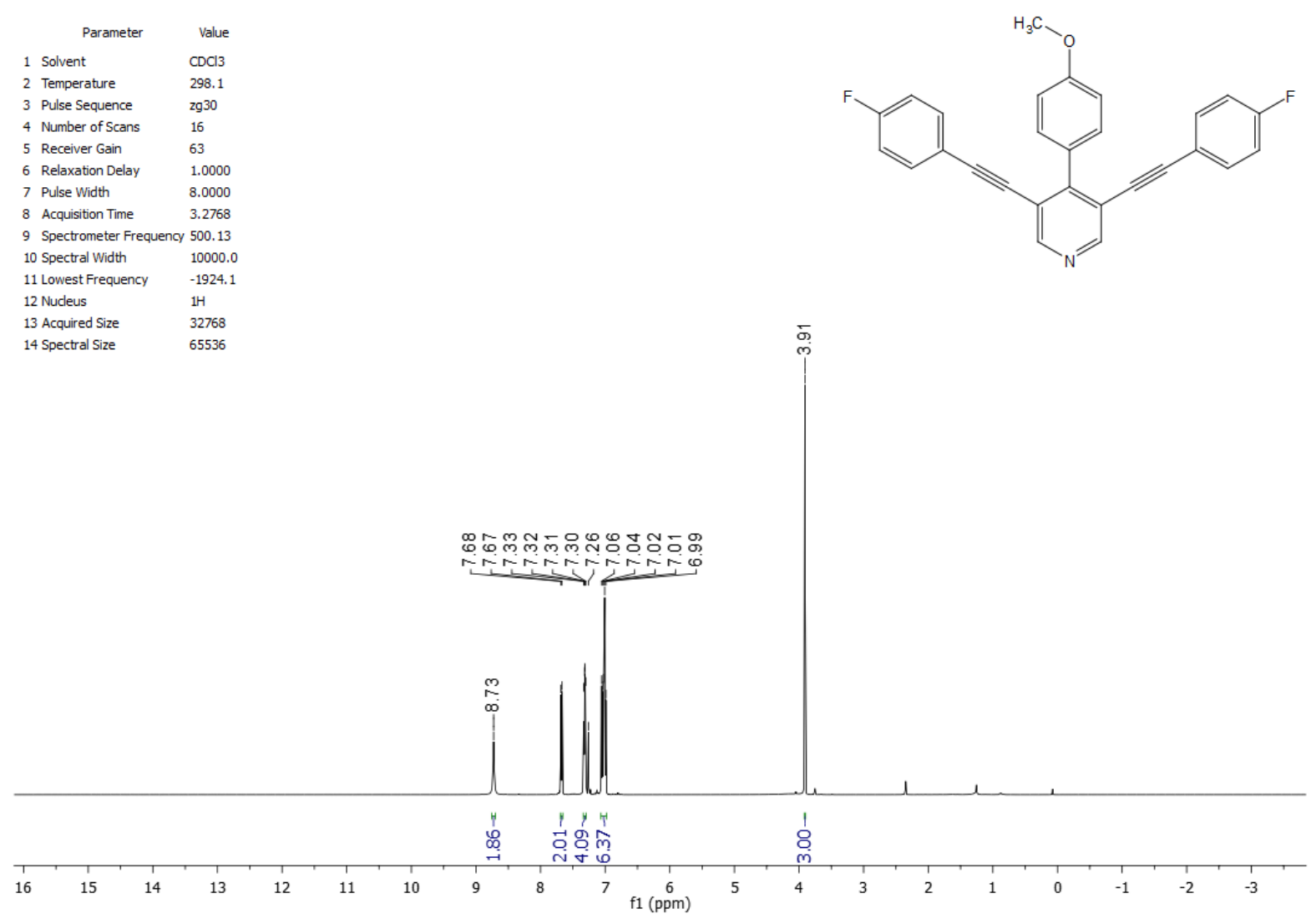

\begin{tabular}{|c|c|c|}
\hline & Parameter & Value \\
\hline & Solvent & $\mathrm{CDCl} 3$ \\
\hline & Temperature & 298.2 \\
\hline & Pulse Sequence & zgig \\
\hline & Number of Scans & 16 \\
\hline & Receiver Gain & 101 \\
\hline & Relaxation Delay & 1.0000 \\
\hline & Pulse Width & 12.0000 \\
\hline & Acquisition Time & 0.5767 \\
\hline & Spectrometer Frequency & 470.59 \\
\hline & Spectral Width & 113636.4 \\
\hline & Lowest Frequency & -103877.4 \\
\hline & Nucleus & $19 \mathrm{~F}$ \\
\hline & Acquired Size & 65536 \\
\hline & Spectral Size & 65536 \\
\hline
\end{tabular}

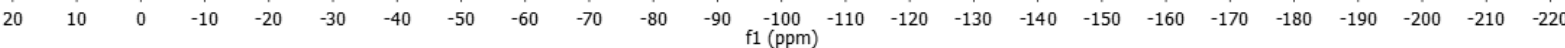




$$
\begin{aligned}
& \text { Parameter Value } \\
& 1 \text { Solvent } \mathrm{CDCl} 3 \\
& 2 \text { Temperature } 298.1 \\
& 3 \text { Pulse Sequence } 2 \text { 2gpg } 30 \\
& 4 \text { Number of Scans } 1024 \\
& 5 \text { Receiver Gain } 101 \\
& 6 \text { Relaxation Delay } \quad 2.0000 \\
& 7 \text { Pulse Width } \quad 10.0000 \\
& 8 \text { Acquisition Time } \quad 1.0879 \\
& 9 \text { Spectrometer Frequency } 125.76 \\
& 10 \text { Spectral Width } \quad 30120.5 \\
& 11 \text { Lowest Frequency } \quad-2489.2 \\
& 12 \text { Nudeus } \quad 13 \mathrm{C} \\
& 13 \text { Acquired Size } \quad 32768 \\
& 14 \text { Spectral Size } \quad 32768
\end{aligned}
$$

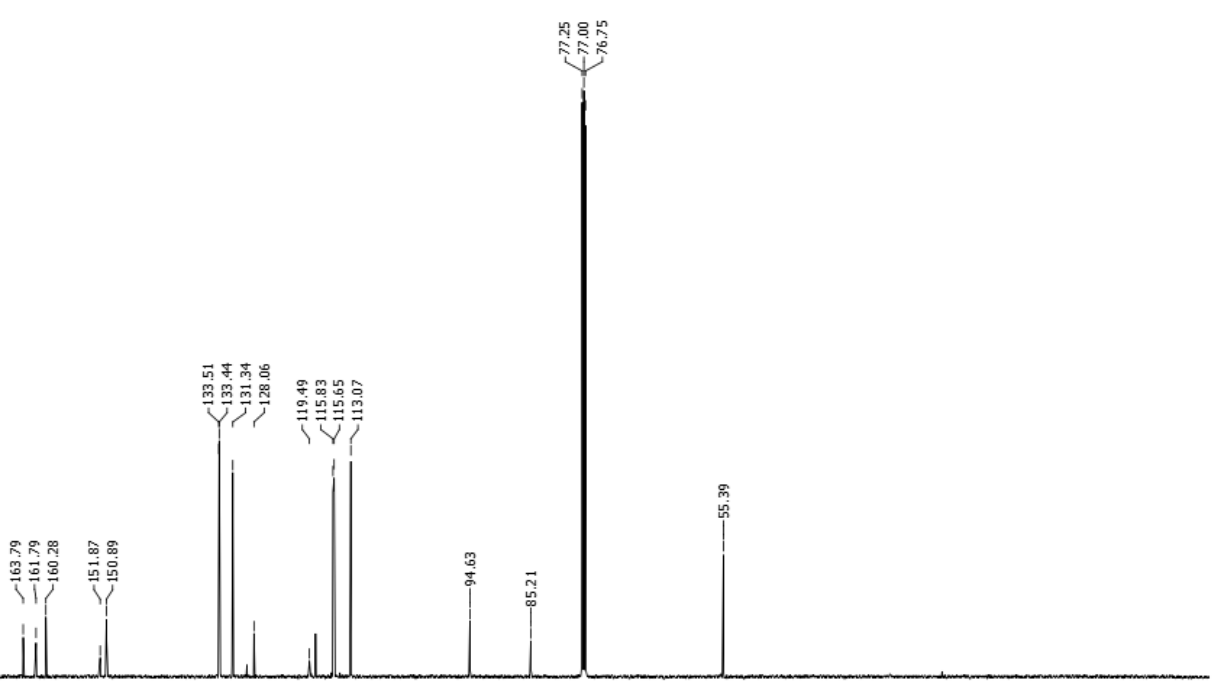

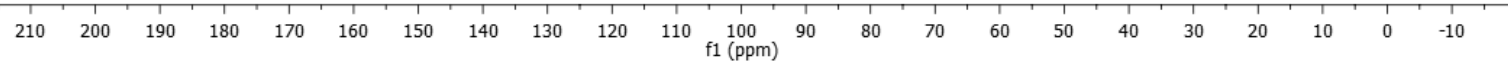


4-phenyl-3,5-bis((4-(trifluoromethyl)phenyl)ethynyl)pyridine (30)

\begin{tabular}{lll}
\multicolumn{1}{c}{ Parameter } & \multicolumn{1}{c}{ Value } \\
1 Solvent & $\mathrm{CDCl} 3$ \\
2 Temperature & 298.1 \\
3 Pulse Sequence & 2930 \\
4 Number of Scans & 16 \\
5 Receiver Gain & 101 \\
6 Relaxation Delay & 1.0000 \\
7 Pulse Width & 8.0000 \\
8 Acquisition Time & 3.2768 \\
9 Spectrometer Frequency & 500.13 \\
10 Spectral Width & 10000.0 \\
11 Lowest Frequency & -1924.4 \\
12 Nucleus & $1 \mathrm{H}$ \\
13 Acquired Size & 32768 \\
14 Spectral Size & 65536
\end{tabular}
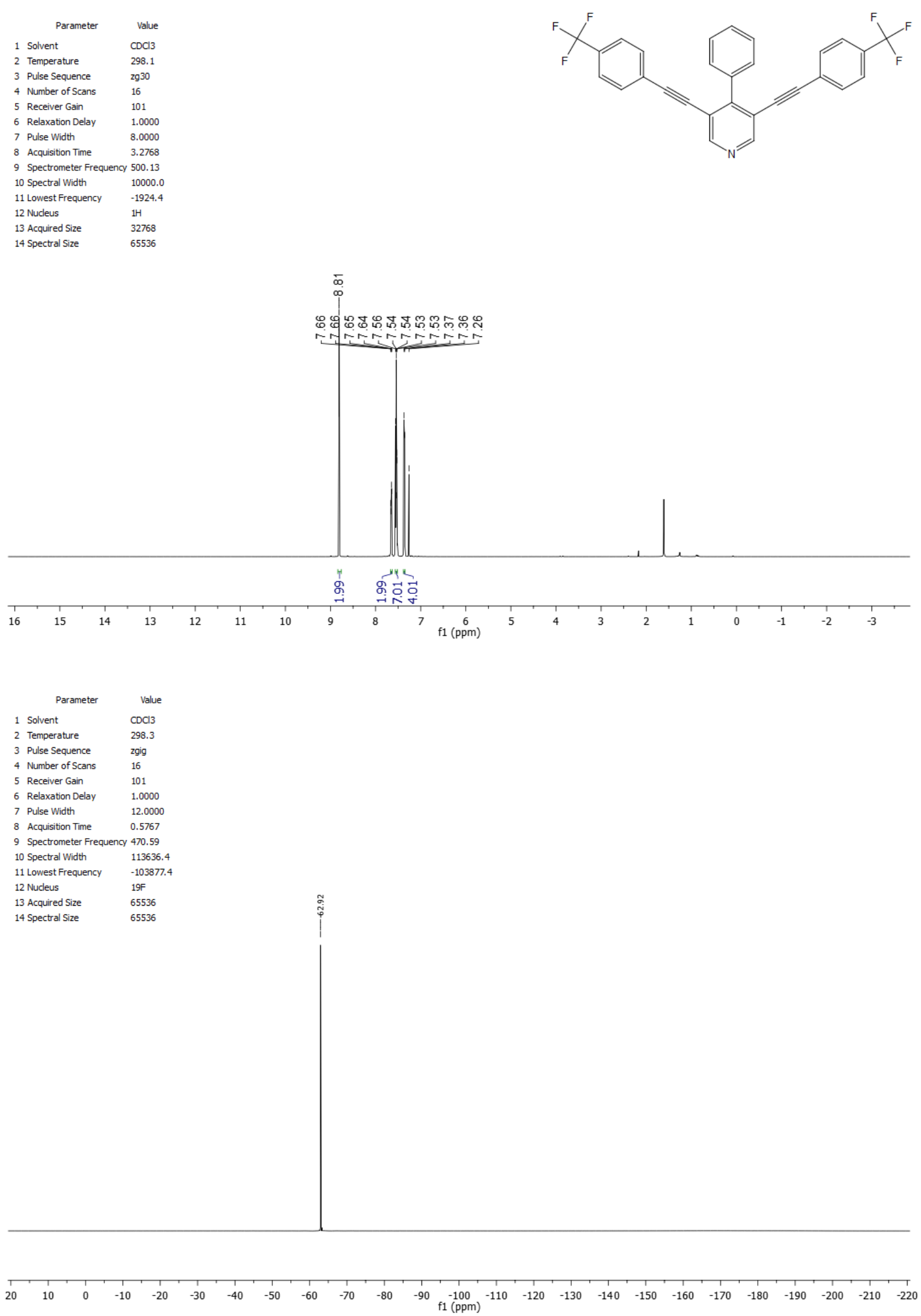

S74 


$$
\begin{array}{lll}
\multicolumn{1}{c}{\text { Parameter }} & \multicolumn{1}{c}{\text { Value }} \\
1 \text { Solvent } & \text { CDCl3 } \\
2 \text { Temperature } & 298.2 \\
3 \text { Pulse Sequence } & \text { 29pg } 30 \\
4 \text { Number of Scans } & 1024 \\
5 \text { Receiver Gain } & 101 \\
6 \text { Relaxation Delay } & 2.0000 \\
7 \text { Pulse Width } & 10.0000 \\
8 \text { Acquisition Time } & 1.0879 \\
9 \text { Spectrometer Frequency } & 125.76 \\
10 \text { Spectral Width } & 30120.5 \\
\text { 11 Lowest Frequency } & -2487.6 \\
\text { 12 Nucleus } & 13 \mathrm{C} \\
\text { 13 Acquired Size } & 32768 \\
\text { 14 Spectral Size } & 32768
\end{array}
$$

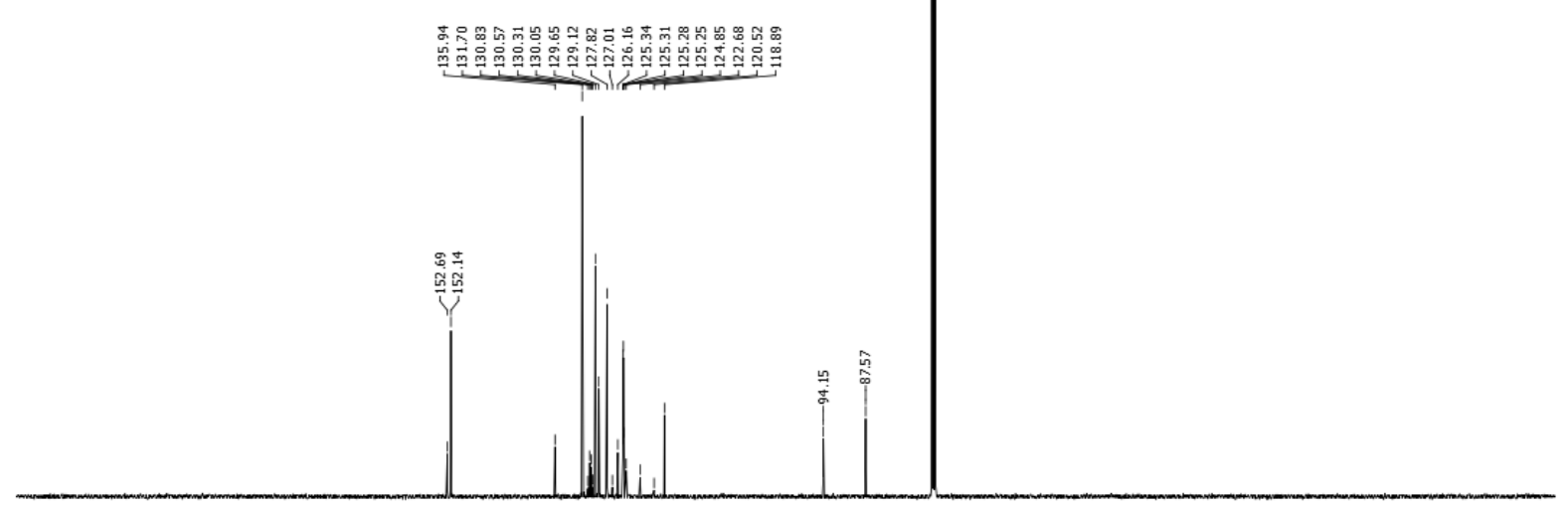

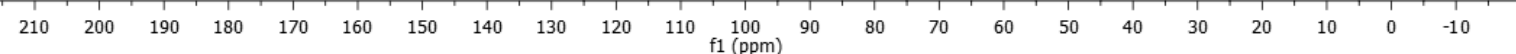


4-(p-tolyl)-3,5-bis((4-(trifluoromethyl)phenyl)ethynyl)pyridine (3p)

\begin{tabular}{lll}
\multicolumn{1}{c}{ Parameter } & \multicolumn{1}{c}{ Value } \\
1 Solvent & $\mathrm{CDCl3}$ \\
2 Temperature & 298.1 \\
3 Pulse Sequence & 2930 \\
4 Number of Scans & 16 \\
5 Receiver Gain & 63 \\
6 Relaxation Delay & 1.0000 \\
7 Pulse Width & 8.0000 \\
8 Acquisition Time & 3.2768 \\
9 Spectrometer Frequency & 500.13 \\
10 Spectral Width & 10000.0 \\
11 Lowest Frequency & -1924.1 \\
12 Nudleus & $1 \mathrm{H}$ \\
13 Acquired Size & 32768 \\
14 Spectral Size & 65536
\end{tabular}
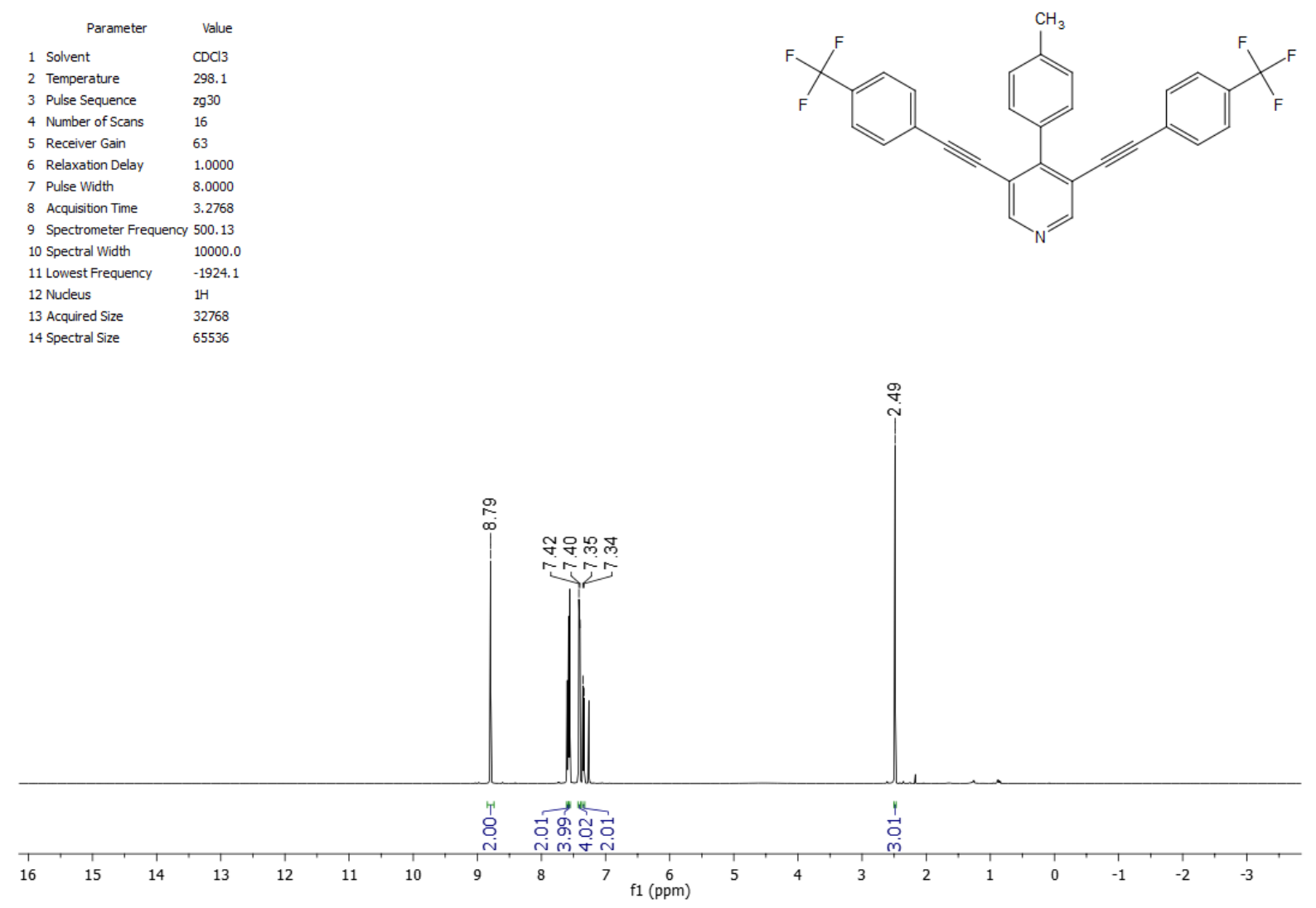

$$
\begin{array}{lll}
\multicolumn{1}{c}{\text { Parameter }} & \multicolumn{1}{c}{\text { Value }} \\
1 \text { Solvent } & \text { CDCl3 } \\
2 \text { 2 } & \text { Temperature } & 298.3 \\
3 \text { Pulse Sequence } & \text { 2gig } \\
4 \text { 4 Number of Scans } & 16 \\
5 \text { Receiver Gain } & 101 \\
6 \text { Relaxation Delay } & 1.0000 \\
7 \text { Pulse Width } & 12.0000 \\
8 \text { Acquisition Time } & 0.5767 \\
9 \text { Spectrometer Frequency } & 470.59 \\
\text { 10 Spectral Width } & 113636.4 \\
\text { 11 Lowest Frequency } & -103877.4 \\
\text { 12 Nudleus } & 19 \mathrm{~F} \\
\text { 13 Acquired Size } & 65536 \\
14 \text { Spectral Size } & 65536
\end{array}
$$




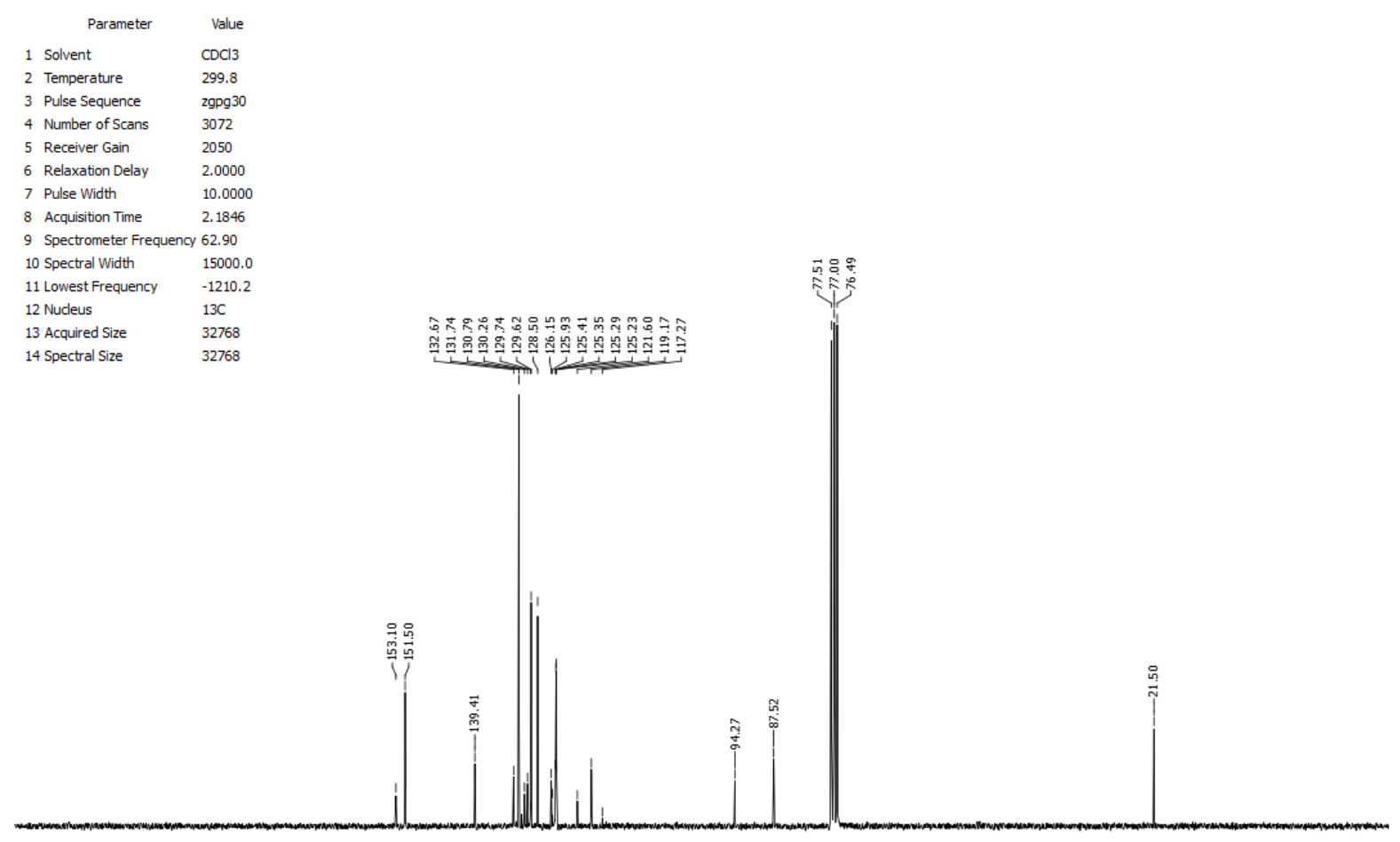

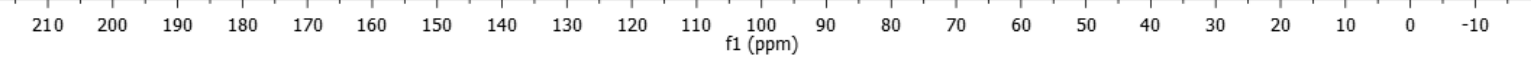


4-(4-methoxyphenyl)-3,5-bis((4-(trifluoromethyl)phenyl)ethynyl)pyridine (3q)

\begin{tabular}{lll}
\multicolumn{1}{c}{ Parameter } & \multicolumn{1}{c}{ Value } \\
1 Solvent & $\mathrm{CDCl3}$ \\
2 Temperature & 298.1 \\
3 Pulse Sequence & $\mathrm{zg} 30$ \\
4 Number of Scans & 16 \\
5 Receiver Gain & 63 \\
6 Relaxation Delay & 1.0000 \\
7 Pulse Width & 8.0000 \\
8 Acquisition Time & 3.2768 \\
9 Spectrometer Frequency & 500.13 \\
10 Spectral Width & 10000.0 \\
11 Lowest Frequency & -1924.0 \\
12 Nucleus & $1 H$ \\
13 Acquired Size & 32768 \\
14 Spectral Size & 65536
\end{tabular}
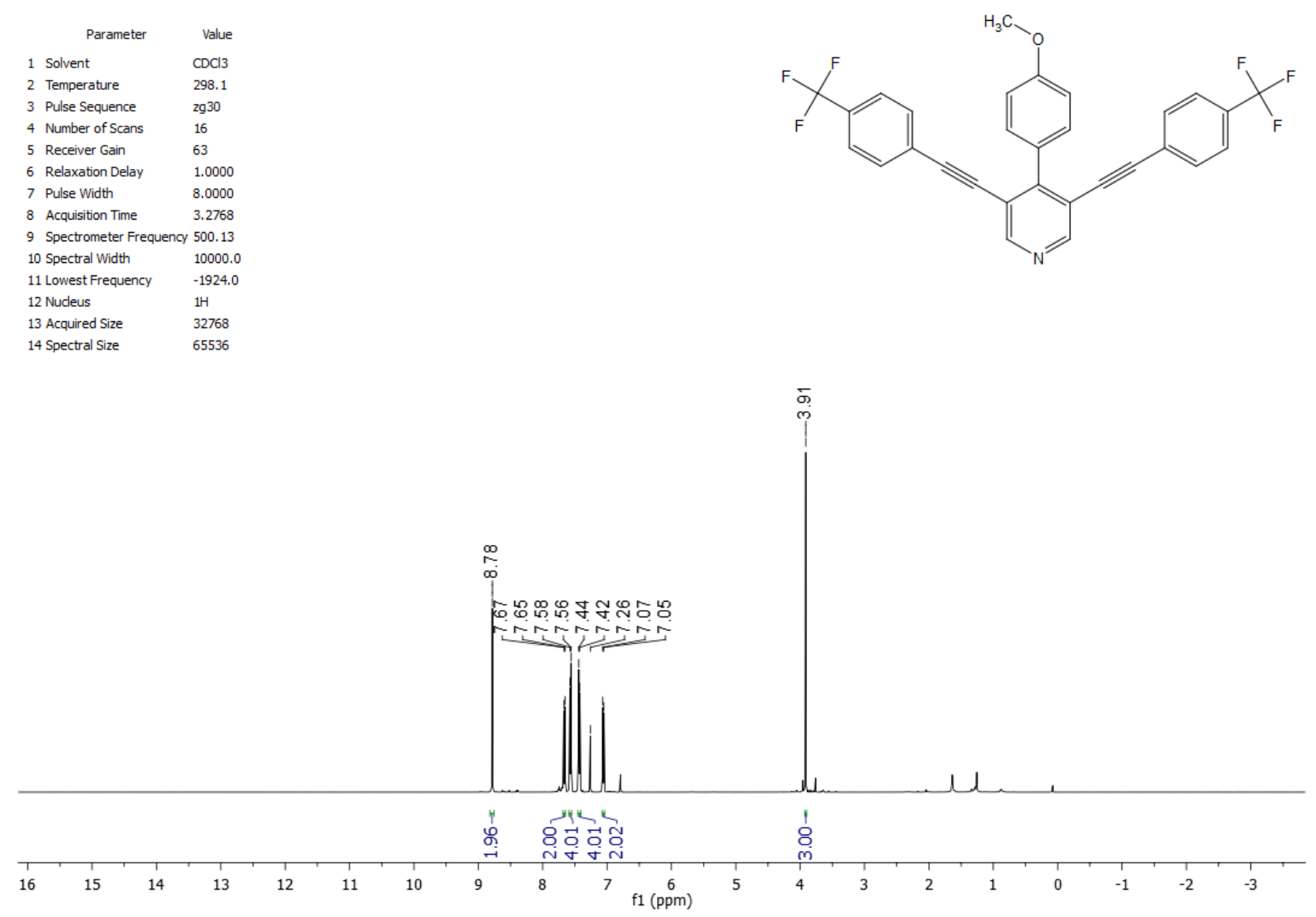

$$
\begin{array}{lll}
\multicolumn{1}{c}{\text { Parameter }} & \multicolumn{1}{c}{\text { Value }} \\
1 \text { Solvent } & \text { CDCl3 } \\
2 \text { Temperature } & 298.3 \\
3 \text { 3 } & \text { Pulse Sequence } & \text { zgig } \\
4 \text { Number of Scans } & 16 \\
5 \text { Receiver Gain } & 101 \\
6 \text { Relaxation Delay } & 1.0000 \\
7 \text { Pulse Width } & 12.0000 \\
8 \text { Acquisition Time } & 0.5767 \\
9 \text { Spectrometer Frequency } & 470.59 \\
10 \text { Spectral Width } & 113636.4 \\
\text { 11 Lowest Frequency } & -103877.4 \\
\text { 12 Nucleus } & 19 F \\
\text { 13 Acquired Size } & 65536 \\
14 \text { Spectral Size } & 65536
\end{array}
$$




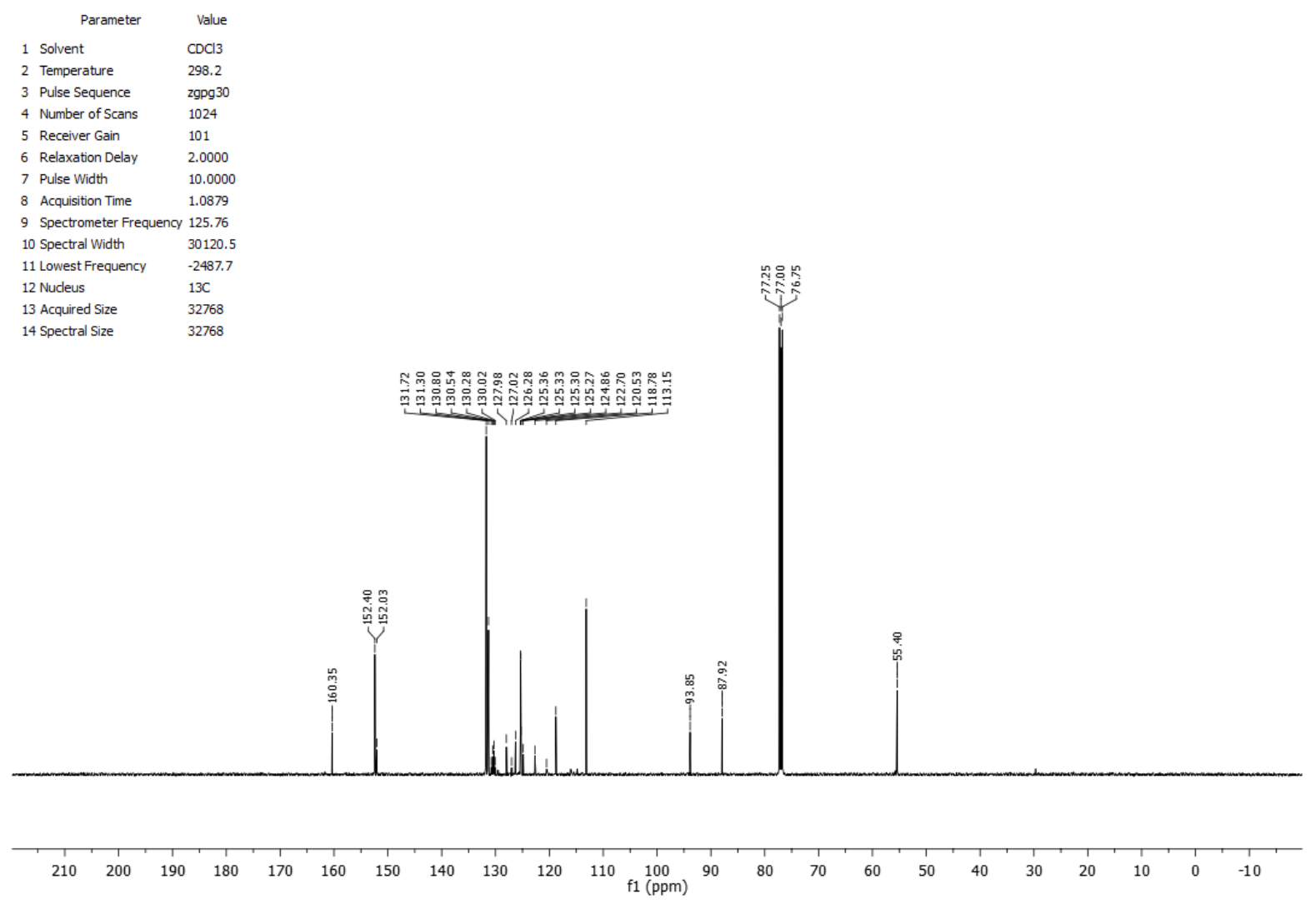




\section{4-phenyl-3,5-bis(thiophen-3-ylethynyl)pyridine (3r)}

\begin{tabular}{lll}
\multicolumn{1}{c}{ Parameter } & \multicolumn{1}{c}{ Value } \\
1 Solvent & CDCl3 \\
2 Temperature & 298.2 \\
3 Pulse Sequence & 2930 \\
4 Number of Scans & 16 \\
5 Receiver Gain & 724 \\
6 Relaxation Delay & 1.0000 \\
7 Pulse Width & 10.0000 \\
8 Acquisition Time & 6.3439 \\
9 Spectrometer Frequency & 250.13 \\
10 Spectral Width & 5165.3 \\
11 Lowest Frequency & -1038.7 \\
12 Nucleus & $1 \mathrm{H}$ \\
13 Acquired Size & 32768 \\
14 Spectral Size & 32768
\end{tabular}
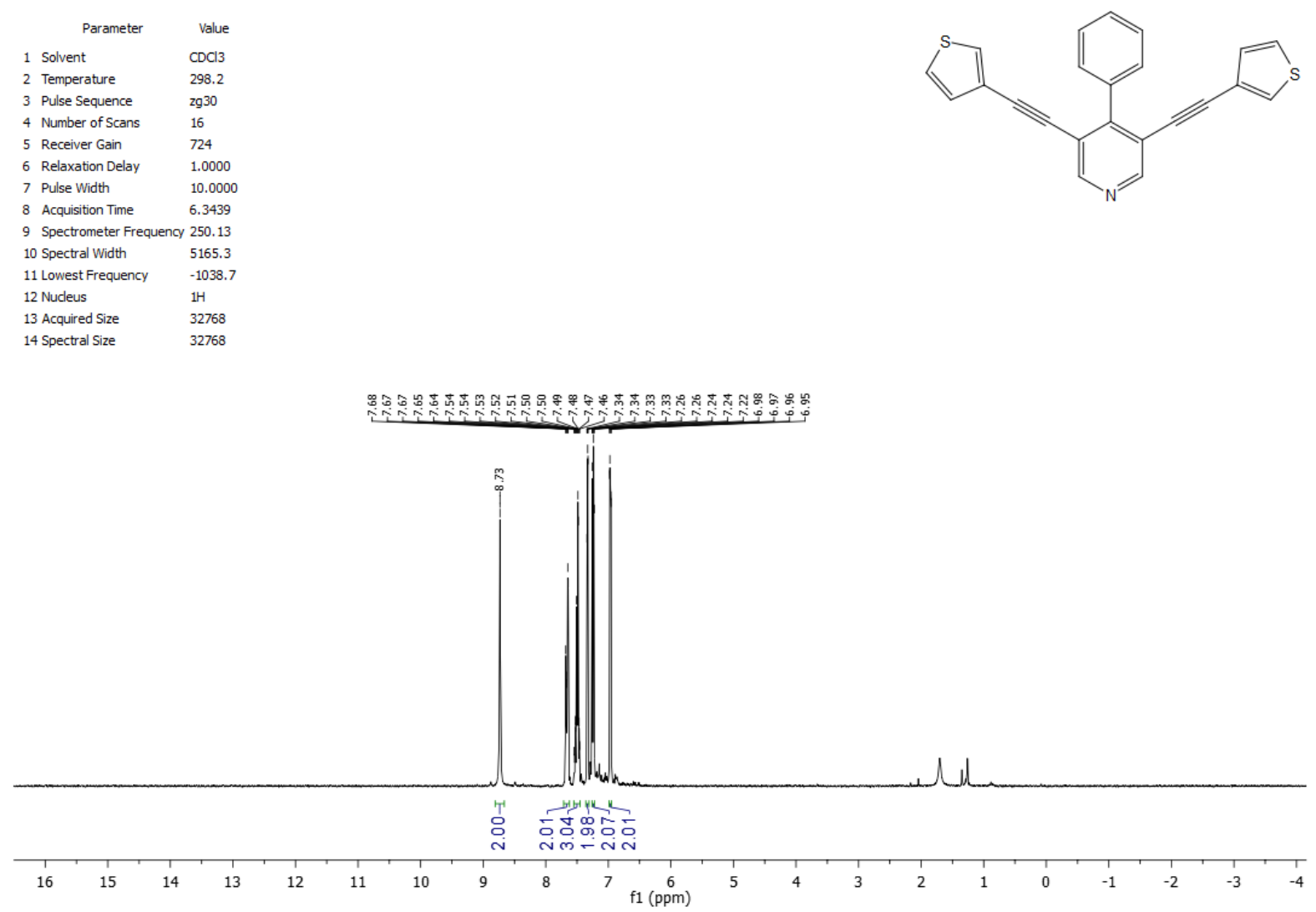

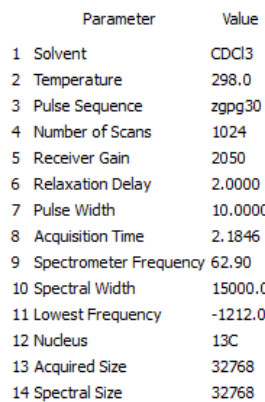

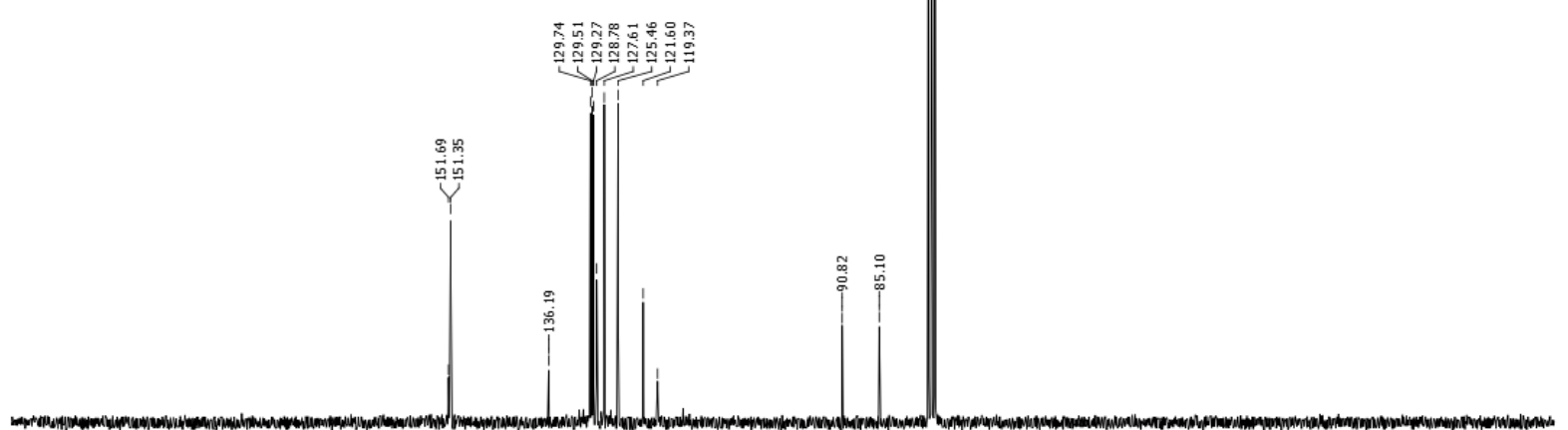

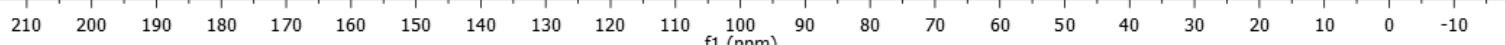




\section{3,5-bis((4-methoxyphenyl)ethynyl)-4-phenylpyridine (3s)}

\begin{tabular}{|c|c|c|}
\hline & Parameter & Valu \\
\hline & Solvent & $\mathrm{CDCl}$ \\
\hline & Temperature & 297.9 \\
\hline & Pulse Sequence & 2930 \\
\hline & Number of Scans & 16 \\
\hline & Receiver Gain & 362 \\
\hline & Relaxation Delay & 1.00 \\
\hline & Pulse Width & 10.0 \\
\hline & Acquisition Time & 6.34 \\
\hline & Spectrometer Frequency & 250 \\
\hline & Spectral Width & 5165 \\
\hline & Lowest Frequency & -103 \\
\hline & Nucleus & $1 \mathrm{H}$ \\
\hline & Acquired Size & \\
\hline & Spectral Size & \\
\hline
\end{tabular}
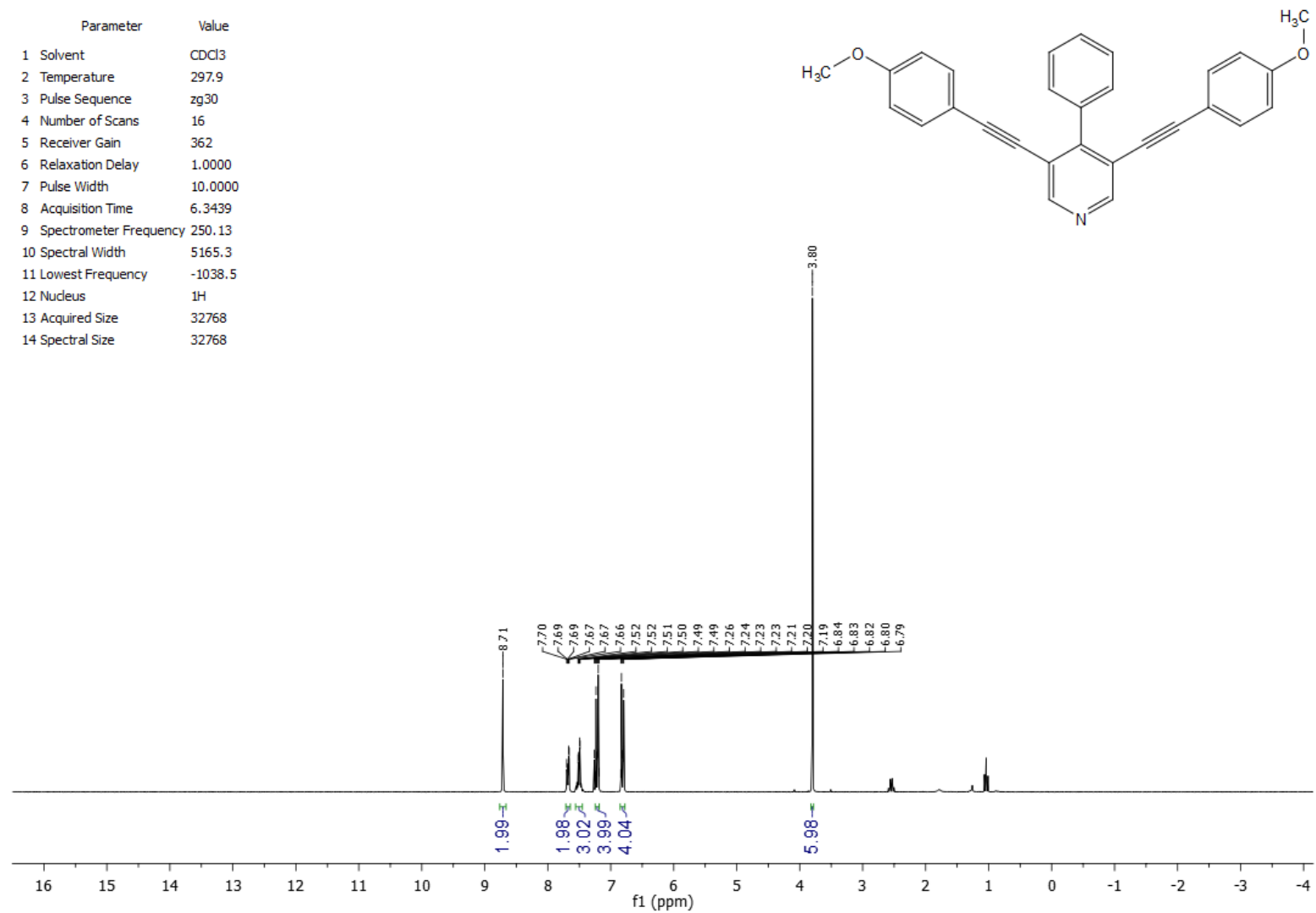

\begin{tabular}{lll}
\multicolumn{1}{c}{ Parameter } & \multicolumn{1}{c}{ Value } \\
1 Solvent & CDCl3 \\
2 2 & Temperature & 297.8 \\
3 Pulse Sequence & 29pg 30 \\
4 Number of Scans & 1024 \\
5 Receiver Gain & 2050 \\
6 Relaxation Delay & 2.0000 \\
7 Pulse Width & 10.0000 \\
8 Acquisition Time & 2.1846 \\
9 Spectrometer Frequency 62.90 \\
10 Spectral Width & 15000.0 \\
11 Lowest Frequency & -1210.9 \\
12 Nucleus & $13 \mathrm{C}$ \\
13 Acquired Size & 32768 \\
14 Spectral Size & 32768
\end{tabular}

14 Spectral Size $\quad 32768$

$\begin{array}{lllllllllll}210 & 200 & 190 & 180 & 170 & 160 & 150 & 140 & 130 & 120 & 110 \\ \mathrm{f} 1(\mathrm{ppm}) & 100\end{array}$ 
4,4'-((4-phenylpyridine-3,5-diyl)bis(ethyne-2,1-diyl))bis( $N, N$-dimethylaniline) (3t)

\begin{tabular}{|c|c|c|}
\hline & Parameter & Value \\
\hline & Solvent & $\mathrm{CDCl} 3$ \\
\hline 27 & Temperature & 298.1 \\
\hline & Pulse Sequence & 2930 \\
\hline & Number of Scans & 16 \\
\hline & Receiver Gain & 101 \\
\hline & Relaxation Delay & 1.0000 \\
\hline & Pulse Width & 8.0000 \\
\hline & Acquisition Time & 3.2768 \\
\hline & Spectrometer Frequency & 500.13 \\
\hline & Spectral Width & 10000.0 \\
\hline & Lowest Frequency & -1924.0 \\
\hline & Nudeus & $1 \mathrm{H}$ \\
\hline & Acquired Size & 32768 \\
\hline & Spectral Size & 65536 \\
\hline
\end{tabular}
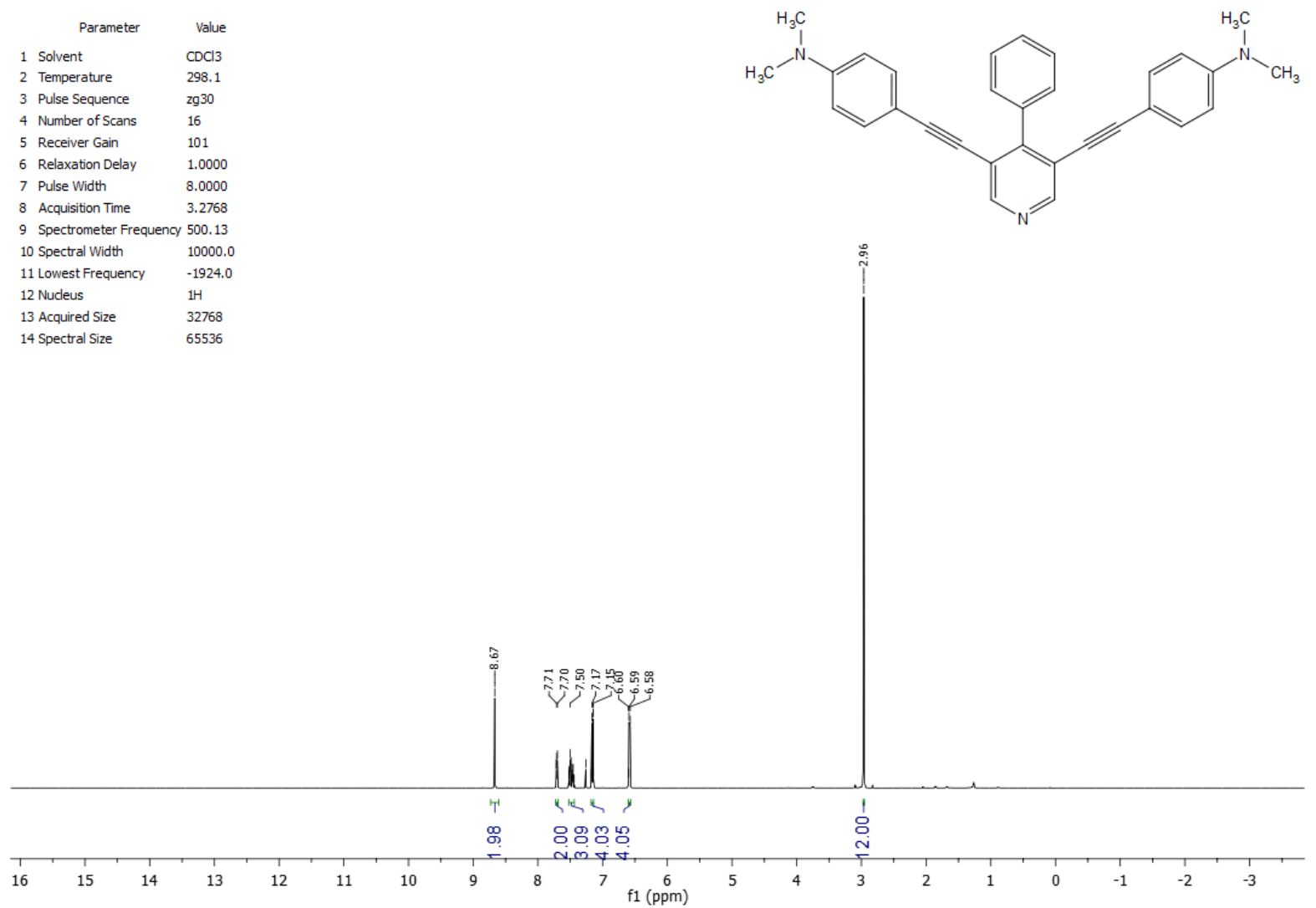

\begin{tabular}{lll} 
& \multicolumn{1}{c}{ Parameter } & \multicolumn{1}{c}{ Value } \\
1 Solvent & CDCl3 \\
2 2 & Temperature & 298.1 \\
3 Pulse Sequence & 29pg 30 \\
4 Number of Scans & 1024 \\
5 Receiver Gain & 101 \\
6 Relaxation Delay & 2.0000 \\
7 Pulse Width & 10.0000 \\
8 Acquisition Time & 1.0879 \\
9 Spectrometer Frequency & 125.76 \\
10 & Spectral Width & 30120.5 \\
11 Lowest Frequency & -2490.9 \\
12 Nucleus & $13 \mathrm{C}$ \\
13 Acquired Size & 32768 \\
14 Spectral Size & 32768
\end{tabular}

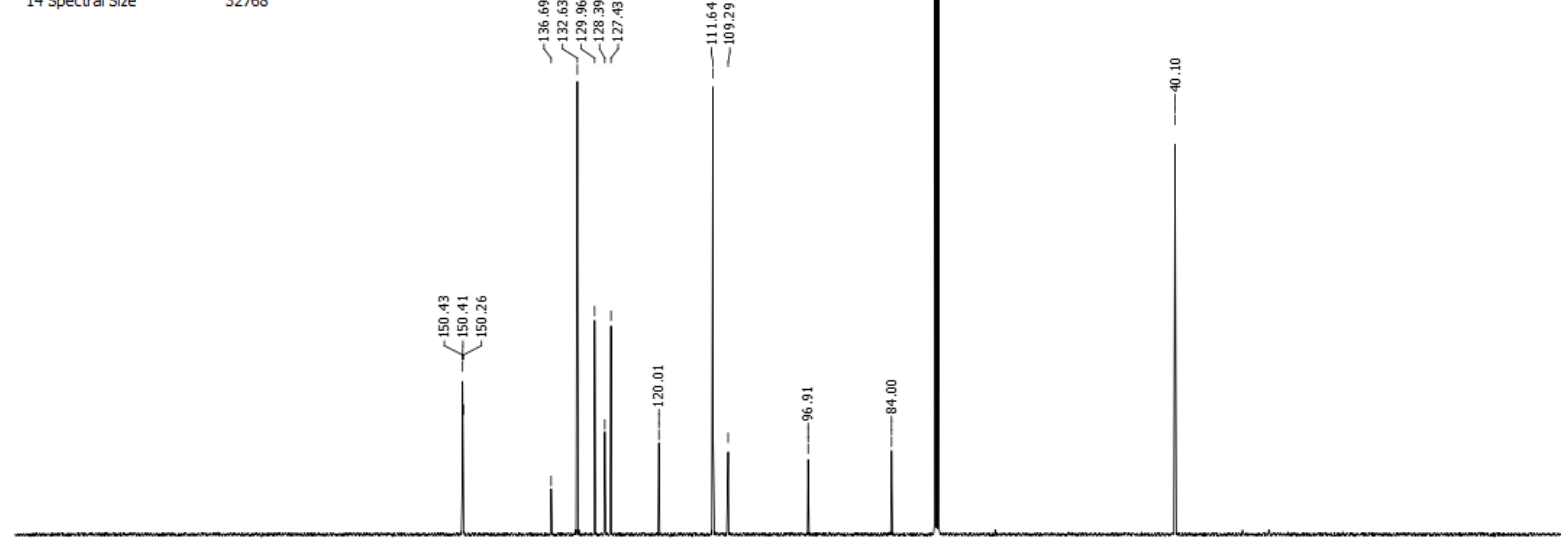

$\begin{array}{lllllllllllllllllllllll}210 & 200 & 190 & 180 & 170 & 160 & 150 & 140 & 130 & 120 & 110 & \underset{\mathrm{f} 1}{100}(\mathrm{ppm}) & 90 & 80 & 70 & 60 & 50 & 40 & 30 & 20 & 10 & 0 & -10\end{array}$ 
6-phenyl-1-(phenylethynyl)benzo[f]isoquinoline (4a)

\begin{tabular}{lll} 
& \multicolumn{1}{c}{ Parameter } & \multicolumn{1}{c}{ Value } \\
1 Solvent & CDCl3 \\
2 Temperature & 298.2 \\
3 Pulse Sequence & $\mathrm{zg} 30$ \\
4 & Number of Scans & 16 \\
5 & Receiver Gain & 362 \\
6 & Relaxation Delay & 1.0000 \\
7 & Pulse Width & 10.0000 \\
8 & Acquisition Time & 6.3439 \\
9 & Spectrometer Frequency 250.13 \\
10 Spectral Width & 5165.3 \\
11 Lowest Frequency & -1038.5 \\
12 Nucleus & $1 \mathrm{H}$ \\
13 Acquired Size & 32768 \\
14 Spectral Size & 32768
\end{tabular}
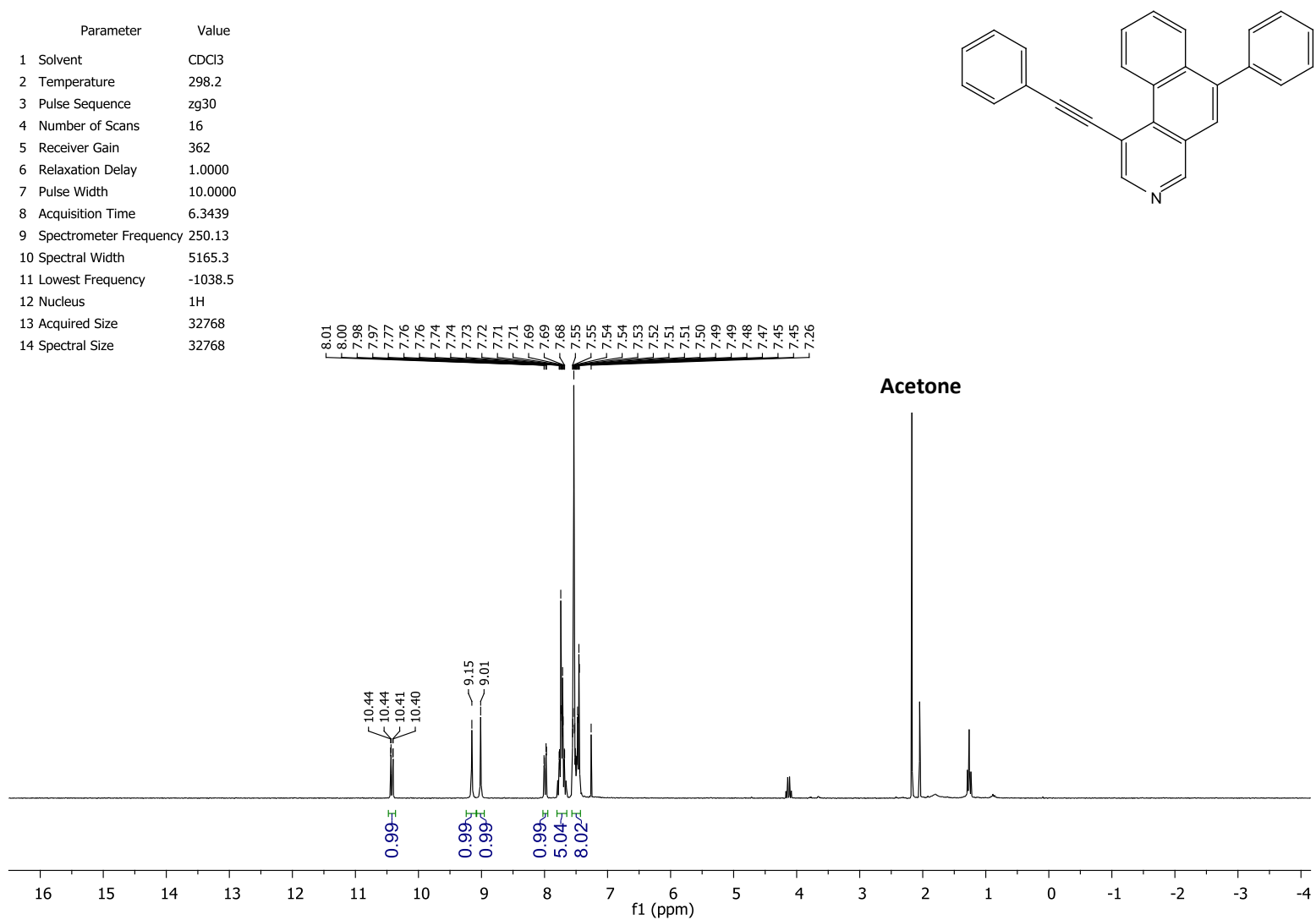

\begin{tabular}{|c|c|c|}
\hline & Parameter & Valu \\
\hline & Solvent & $\mathrm{CDCl}_{3}^{3}$ \\
\hline & Temperature & 298. \\
\hline & Pulse Sequence & zgpg3 3 \\
\hline & Number of Scans & 2048 \\
\hline & Receiver Gain & 2050 \\
\hline & Relaxation Delay & 2.00 \\
\hline & Pulse Width & 10.00 \\
\hline & Acquisition Time & 2.184 \\
\hline & Spectrometer Frequency & 62.90 \\
\hline & Spectral Width & 1500 \\
\hline & Lowest Frequency & -121 \\
\hline & Nudeus & $13 \mathrm{C}$ \\
\hline & Acquired Size & 327 \\
\hline & Spectral Size & 3276 \\
\hline
\end{tabular}

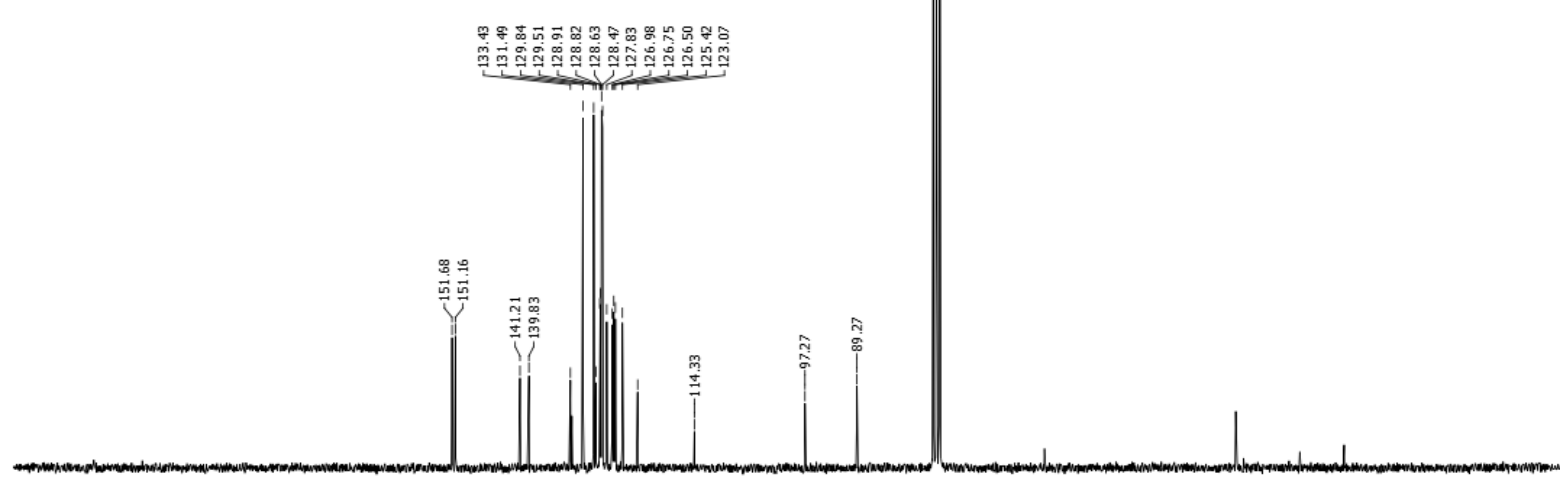

$\begin{array}{lllllllllllllllllllllll}210 & 200 & 190 & 180 & 170 & 160 & 150 & 140 & 130 & 120 & 110 & 100 & 90 & 80 & 70 & 60 & 50 & 40 & 30 & 20 & 10 & 0 & -10\end{array}$ 


\section{8-methyl-6-phenyl-1-(phenylethynyl)benzo[ $f$ isoquinoline (4b)}

\begin{tabular}{lll}
\multicolumn{1}{c}{ Parameter } & \multicolumn{1}{c}{ Value } \\
1 Solvent & CDCl3 \\
2 Temperature & 298.2 \\
3 Pulse Sequence & \multicolumn{2}{c}{2930} \\
4 Number of Scans & 16 \\
5 Receiver Gain & 362 \\
6 Relaxation Delay & 1.0000 \\
7 Pulse Width & 10.0000 \\
8 Acquisition Time & 6.3439 \\
9 Spectrometer Frequency 250.13 \\
10 Spectral Width & 5165.3 \\
11 Lowest Frequency & -1038.5 \\
12 Nucleus & $1 \mathrm{H}$ \\
13 Acquired Size & 32768 \\
14 Spectral Size & 32768
\end{tabular}
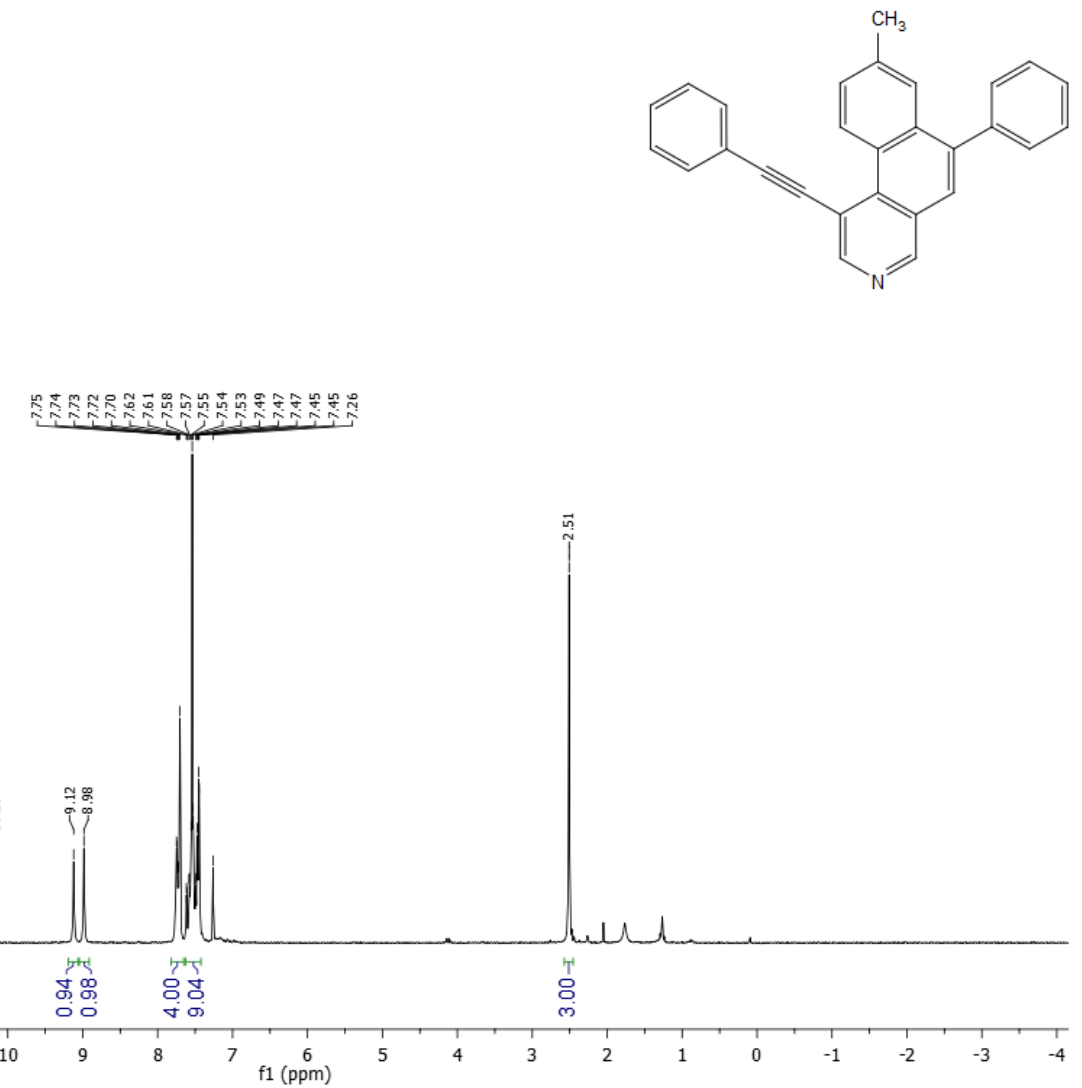

\begin{tabular}{|c|c|c|}
\hline & Parameter & \\
\hline & Solvent & $\mathrm{CDCl}$ \\
\hline & Temperature & 298. \\
\hline & Pulse Sequence & $2 g p g$ \\
\hline & Number of Scans & 1024 \\
\hline & Receiver Gain & 2050 \\
\hline & Relaxation Delay & 2.00 \\
\hline & Pulse Width & 10.0 \\
\hline & Acquisition Time & 2.1846 \\
\hline \multicolumn{3}{|c|}{ Spectrometer Frequency 62.90} \\
\hline & Spectral Width & 1500 \\
\hline & Lowest Frequency & -121 \\
\hline & Nucleus & $13 \mathrm{C}$ \\
\hline & Acquired Size & 3276 \\
\hline & & 327 \\
\hline
\end{tabular}

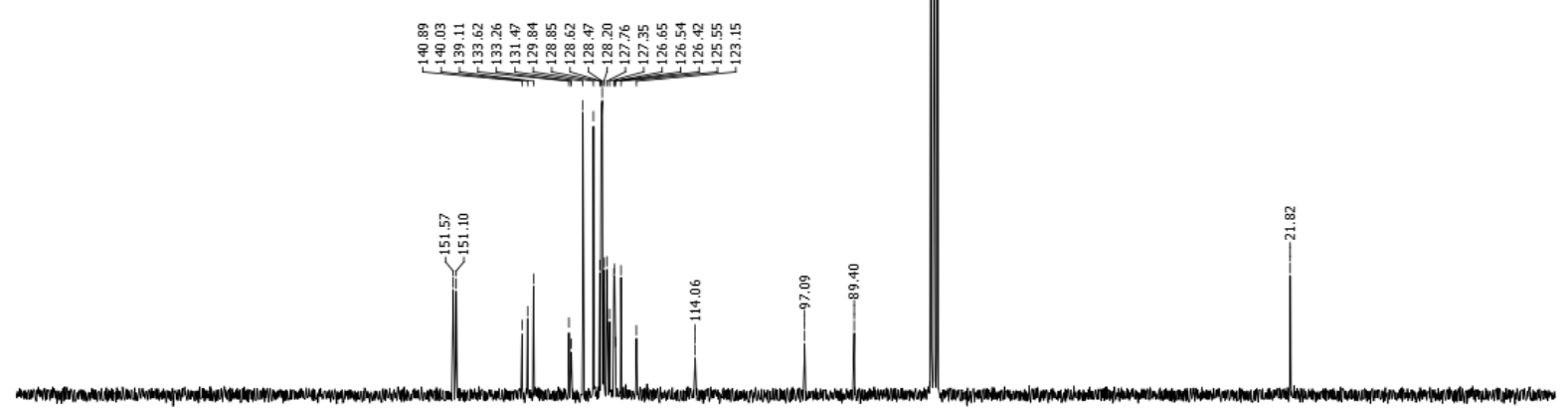

$\begin{array}{lllllllllll}210 & 200 & 190 & 180 & 170 & 160 & 150 & 140 & 130 & 120 & 110 \underset{\mathrm{f} 1(\mathrm{ppm})}{100}\end{array}$ 


\section{6-phenyl-1-(phenylethynyl)-8-(trifluoromethyl)benzo[f]isoquinoline (4c)}

\begin{tabular}{lll}
\multicolumn{1}{c}{ Parameter } & \multicolumn{1}{c}{ Value } \\
1 Solvent & $\mathrm{CDCl} 3$ \\
2 Temperature & 298.2 \\
3 Pulse Sequence & 2930 \\
4 N & Number of Scans & 16 \\
5 Receiver Gain & 63 \\
6 Relaxation Delay & 1.0000 \\
7 Pulse Width & 8.0000 \\
8 Acquisition Time & 3.2768 \\
9 Spectrometer Frequency & 500.13 \\
10 Spectral Width & 10000.0 \\
11 Lowest Frequency & -1924.1 \\
12 Nucleus & $1 \mathrm{H}$ \\
13 Acquired Size & 32768 \\
14 Spectral Size & 65536
\end{tabular}
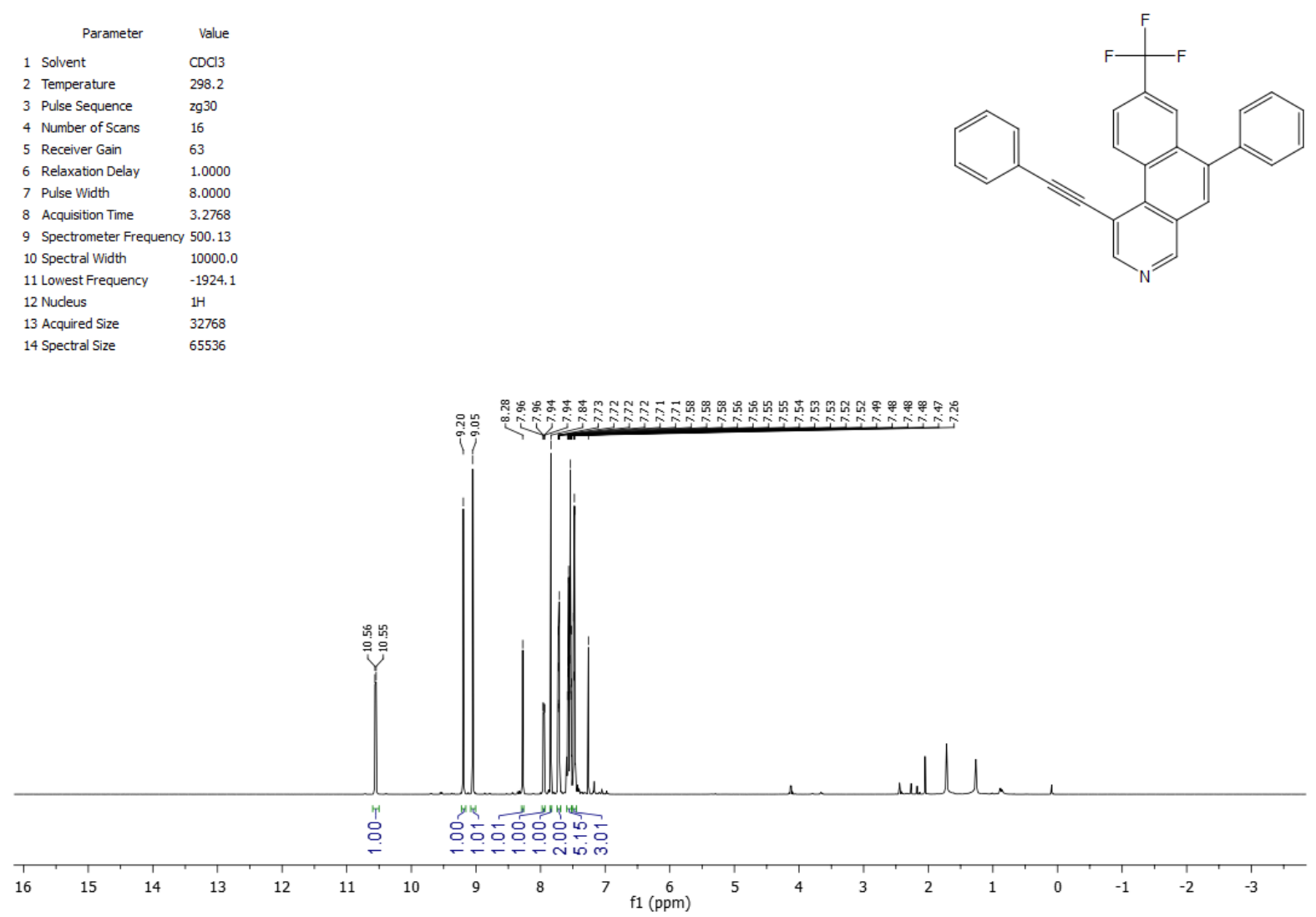

\begin{tabular}{lll}
\multicolumn{1}{c}{ Parameter } & \multicolumn{1}{c}{ Value } \\
1 Solvent & $\mathrm{CDCl} 3$ \\
2 Temperature & 298.2 \\
3 Pulse Sequence & zgig \\
4 Number of Scans & 16 \\
5 Receiver Gain & 101 \\
6 Relaxation Delay & 1.0000 \\
7 Pulse Width & 12.0000 \\
8 Acquisition Time & 0.5767 \\
9 Spectrometer Frequency & 470.59 \\
10 Spectral Width & 113636.4 \\
11 Lowest Frequency & -103877.4 \\
12 Nucleus & $19 \mathrm{~F}$ \\
13 Acquired Size & 65536 \\
14 Spectral Size & 65536
\end{tabular}

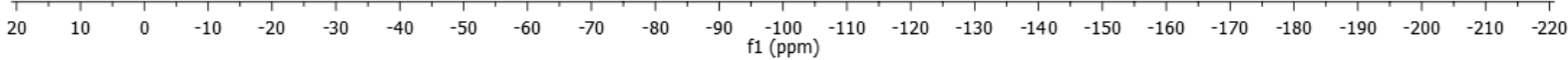




$$
\begin{aligned}
& \begin{array}{llr} 
& \multicolumn{1}{c}{\text { Parameter }} & \text { Value } \\
1 \text { Solvent } & \text { CDCl3 } \\
2 \text { 2 } & \text { Temperature } & 298.2
\end{array} \\
& 3 \text { Pulse Sequence } 29 \mathrm{pg} 30 \\
& 3 \text { Pulse Sequence } 29 p 930 \\
& \begin{array}{lll}
4 & \text { Number of Scans } & 1024 \\
5 & \text { Receiver Gain } & 101
\end{array} \\
& \begin{array}{lll}
5 & \text { Receiver Gain } & 101 \\
6 & \text { Relaxation Delay } & 2.0000
\end{array} \\
& 7 \text { Pulse Width } 10.000 \\
& 8 \text { Acquisition Time } \quad 1.0879 \\
& 9 \text { Spectrometer Frequency } 125.76 \\
& 10 \text { Spectral Width } \quad 30120.5
\end{aligned}
$$

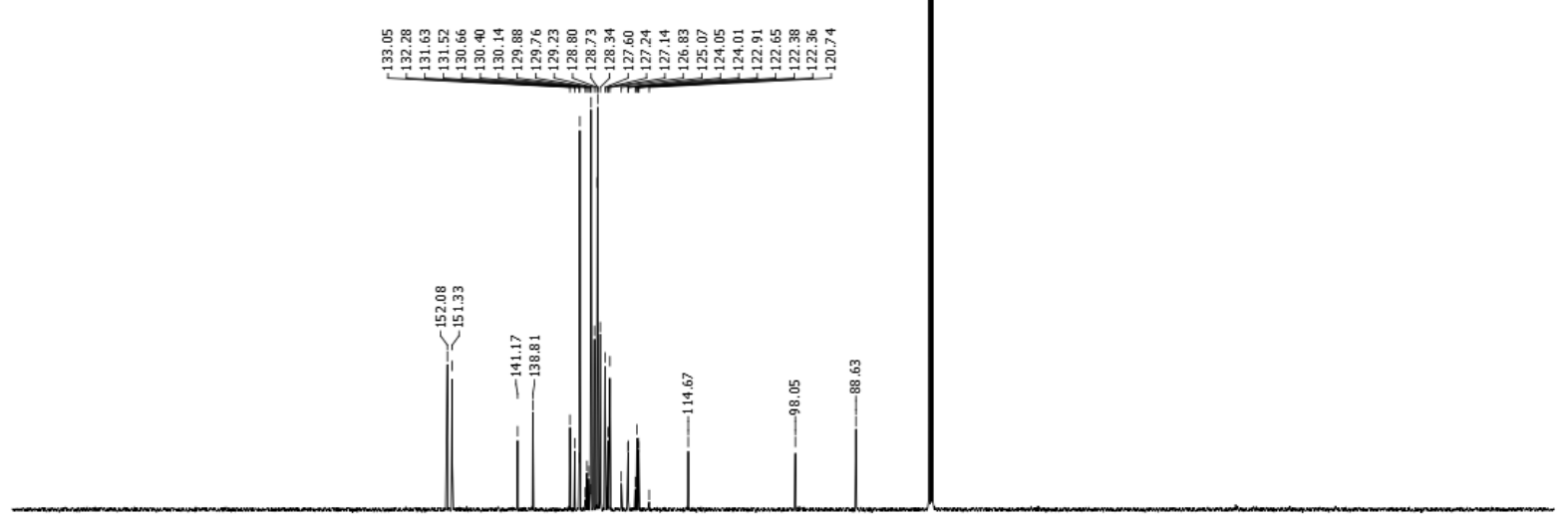

$\begin{array}{llllllllllllllllllllllllll}210 & 200 & 190 & 180 & 170 & 160 & 150 & 140 & 130 & 120 & 110 & 100 & 90 & 80 & 70 & 60 & 50 & 40 & 30 & 20 & 10 & 0 & -10\end{array}$ 
6-(p-tolyl)-1-(p-tolylethynyl)benzo[ $f$ isoquinoline (4d)

\begin{tabular}{lll}
\multicolumn{1}{c}{ Parameter } & \multicolumn{1}{c}{ Value } \\
1 Solvent & CDCl3 \\
2 & Temperature & 298.2 \\
3 & Pulse Sequence & 2930 \\
4 Number of Scans & 16 \\
5 Seceiver Gain & 575 \\
6 Relaxation Delay & 1.0000 \\
7 Pulse Width & 10.0000 \\
8 Acquisition Time & 6.3439 \\
9 Spectrometer Frequency 250.13 \\
10 Spectral Width & 5165.3 \\
11 Lowest Frequency & -1061.7 \\
12 Nucleus & $1 \mathrm{H}$ \\
13 Acquired Size & 32768 \\
14 Spectral Size & 32768
\end{tabular}
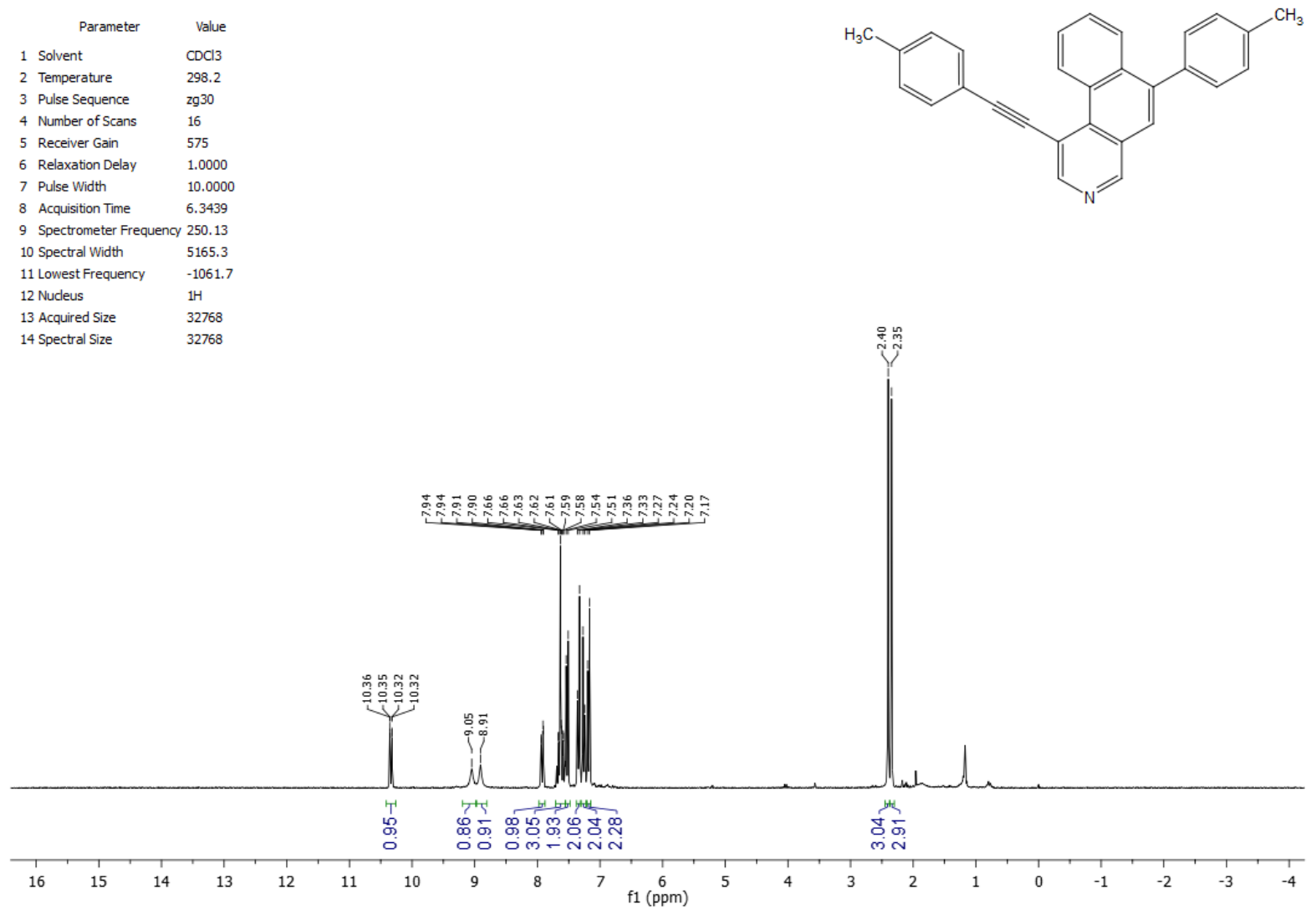

\begin{tabular}{lll}
\multicolumn{1}{c}{ Parameter } & \multicolumn{1}{c}{ Value } \\
1 Solvent & CDCl3 \\
2 Temperature & 298.0 \\
3 Pulse Sequence & zgpg 30 \\
4 Number of Scans & 2048 \\
5 5eceiver Gain & 2050 \\
6 Relaxation Delay & 2.0000 \\
7 Pulse Width & 10.0000 \\
8 Acquisition Time & 2.1846 \\
9 Spectrometer Frequency & 62.90 \\
10 Spectral Width & 15000.0 \\
11 Lowest Frequency & -1212.0 \\
12 Nucleus & $13 \mathrm{C}$ \\
13 Acquired Size & 32768 \\
14 Spectral Size & 32768
\end{tabular}

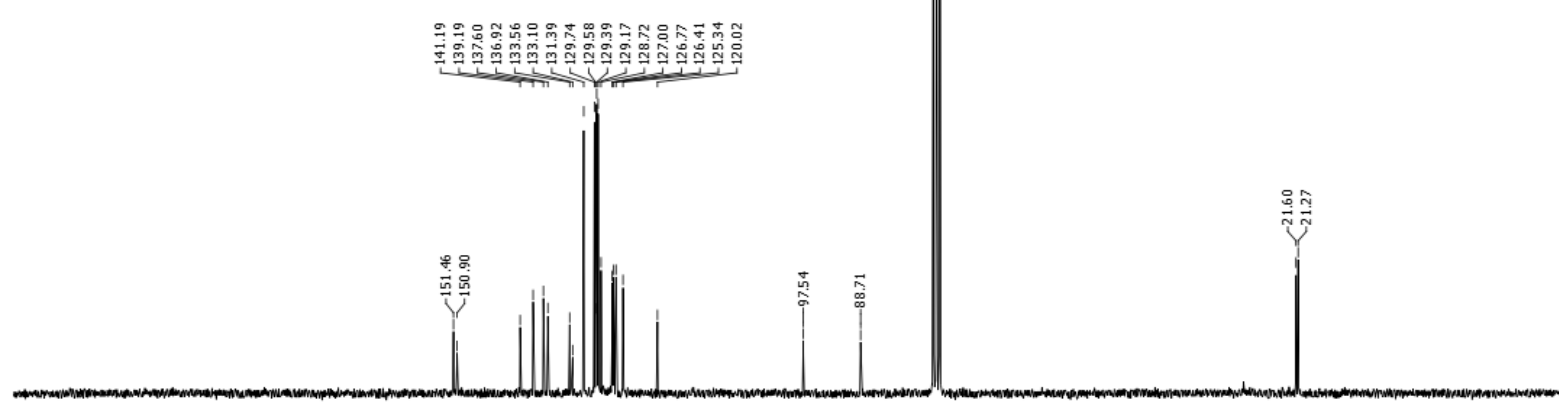

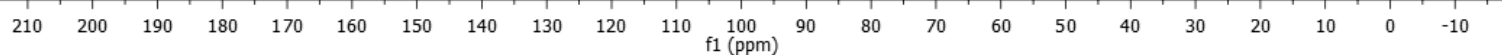


8-methyl-6-(p-tolyl)-1-(p-tolylethynyl)benzo[f]isoquinoline (4e)

\begin{tabular}{lll}
\multicolumn{1}{c}{ Parameter } & \multicolumn{1}{c}{ Value } \\
1 Solvent & $\mathrm{CDCl} 3$ \\
2 2emperature & 298.2 \\
3 Pulse Sequence & $\mathrm{zg} 30$ \\
4 Number of Scans & 16 \\
5 Receiver Gain & 144 \\
6 Relaxation Delay & 1.0000 \\
7 Pulse Width & 10.0000 \\
8 Acquisition Time & 5.2954 \\
9 Spectrometer Frequency & 300.13 \\
10 & Spectral Width & 6188.1 \\
11 Lowest Frequency & -1246.3 \\
12 Nucleus & $1 \mathrm{H}$ \\
13 Acquired Size & 32768 \\
14 Spectral Size & 32768
\end{tabular}
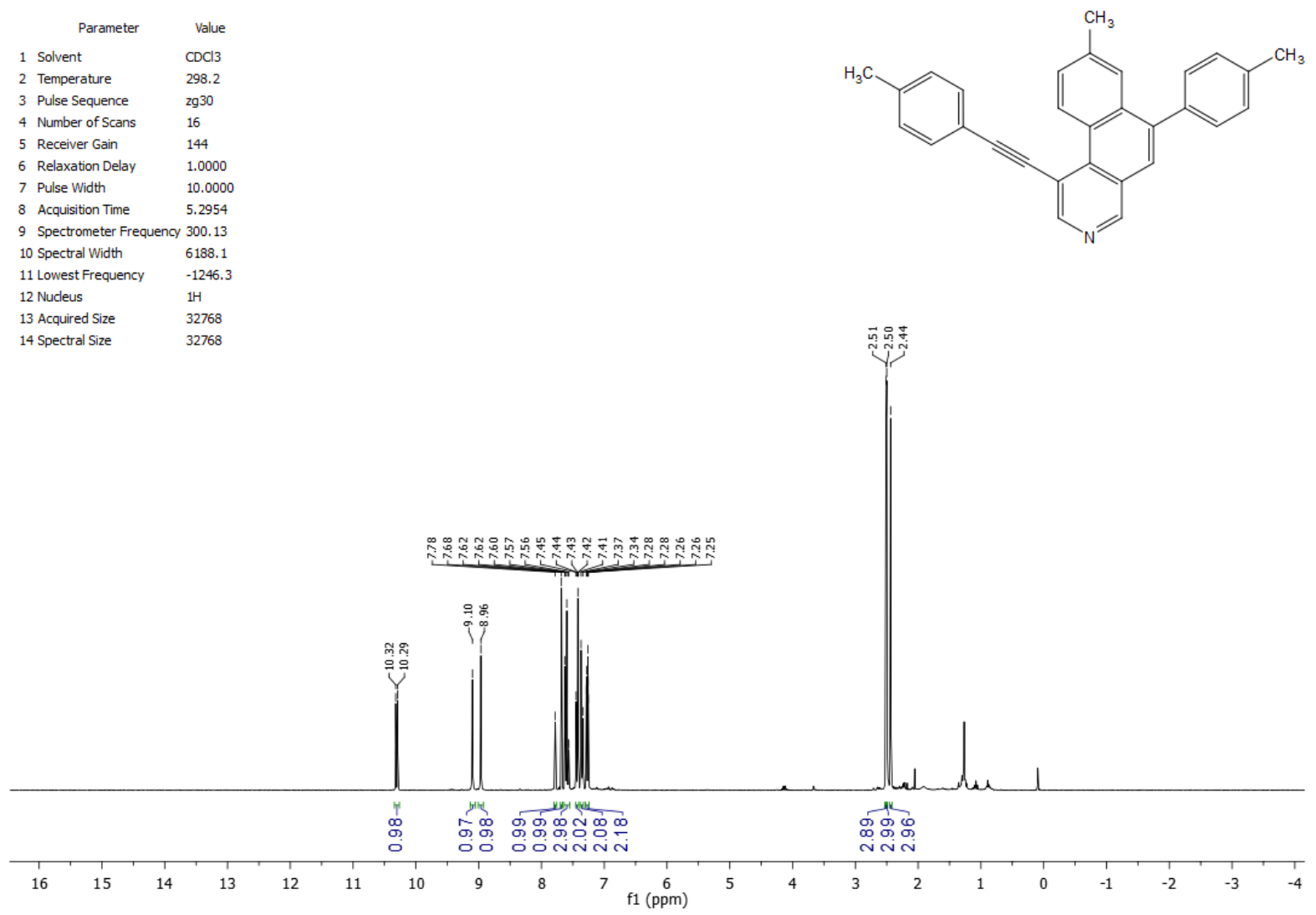

\begin{tabular}{lll}
\multicolumn{1}{c}{ Parameter } & \multicolumn{1}{c}{ Value } \\
1 Solvent & CDCl3 \\
2 & Temperature & 298.2 \\
3 Pulse Sequence & 29pg30 \\
4 Number of Scans & 1024 \\
5 Receiver Gain & 2050 \\
6 Relaxation Delay & 2.0000 \\
7 Pulse Width & 10.0000 \\
8 Acquisition Time & 1.8176 \\
9 Spectrometer Frequency 75.47 \\
10 Spectral Width & 18028.8 \\
11 Lowest Frequency & -1470.9 \\
12 Nucleus & $13 \mathrm{C}$ \\
13 Acquired Size & 32768 \\
14 Spectral Size & 32768
\end{tabular}

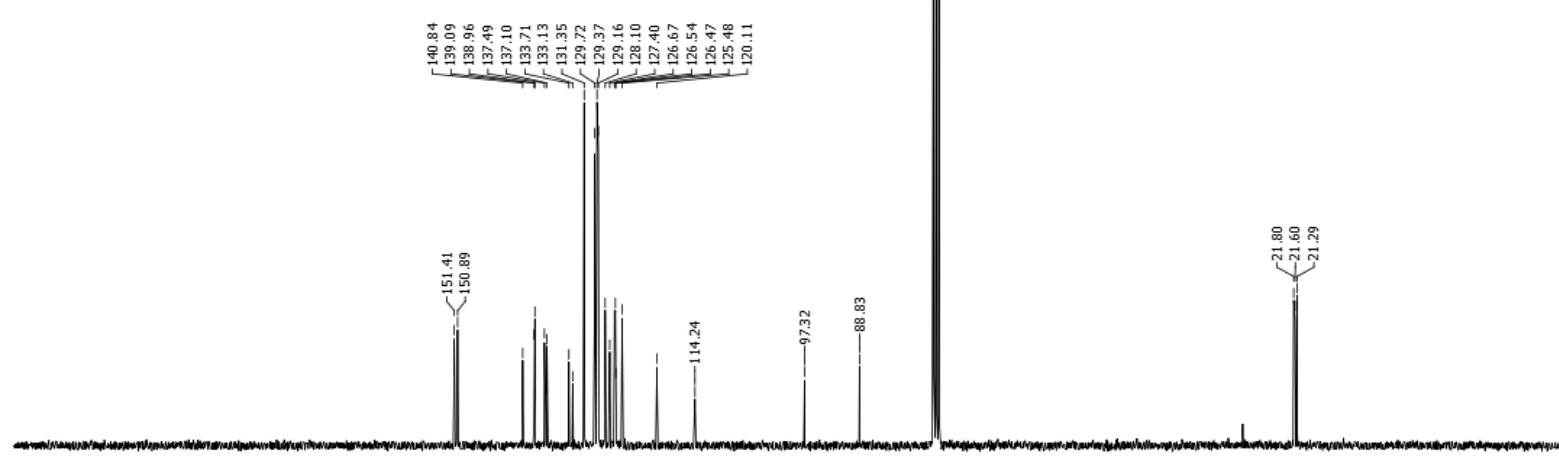

$\begin{array}{lllllllllllllllllllllll}210 & 200 & 190 & 180 & 170 & 160 & 150 & 140 & 130 & 120 & 110 & 100 & 90 & 80 & 70 & 60 & 50 & 40 & 30 & 20 & 10 & 0 & -10\end{array}$ 
6-(p-tolyl)-1-( $p$-tolylethynyl)-8-(trifluormethyl)benzo[f]isoquinoline (4f)

\begin{tabular}{lll}
\multicolumn{1}{c}{ Parameter } & \multicolumn{1}{c}{ Value } \\
1 Solvent & CDCl3 \\
2 & Temperature & 298.2 \\
3 & Pulse Sequence & 2930 \\
4 Number of Scans & 16 \\
5 S & Receiver Gain & 144 \\
6 Relaxation Delay & 1.0000 \\
7 7 & Pulse Width & 10.0000 \\
8 & Acquisition Time & 5.2954 \\
9 Spectrometer Frequency & 300.13 \\
10 & Spectral Width & 6188.1 \\
11 Lowest Frequency & -1246.6 \\
12 Nudeus & $1 \mathrm{H}$ \\
13 Acquired Size & 32768 \\
14 Spectral Size & 32768
\end{tabular}
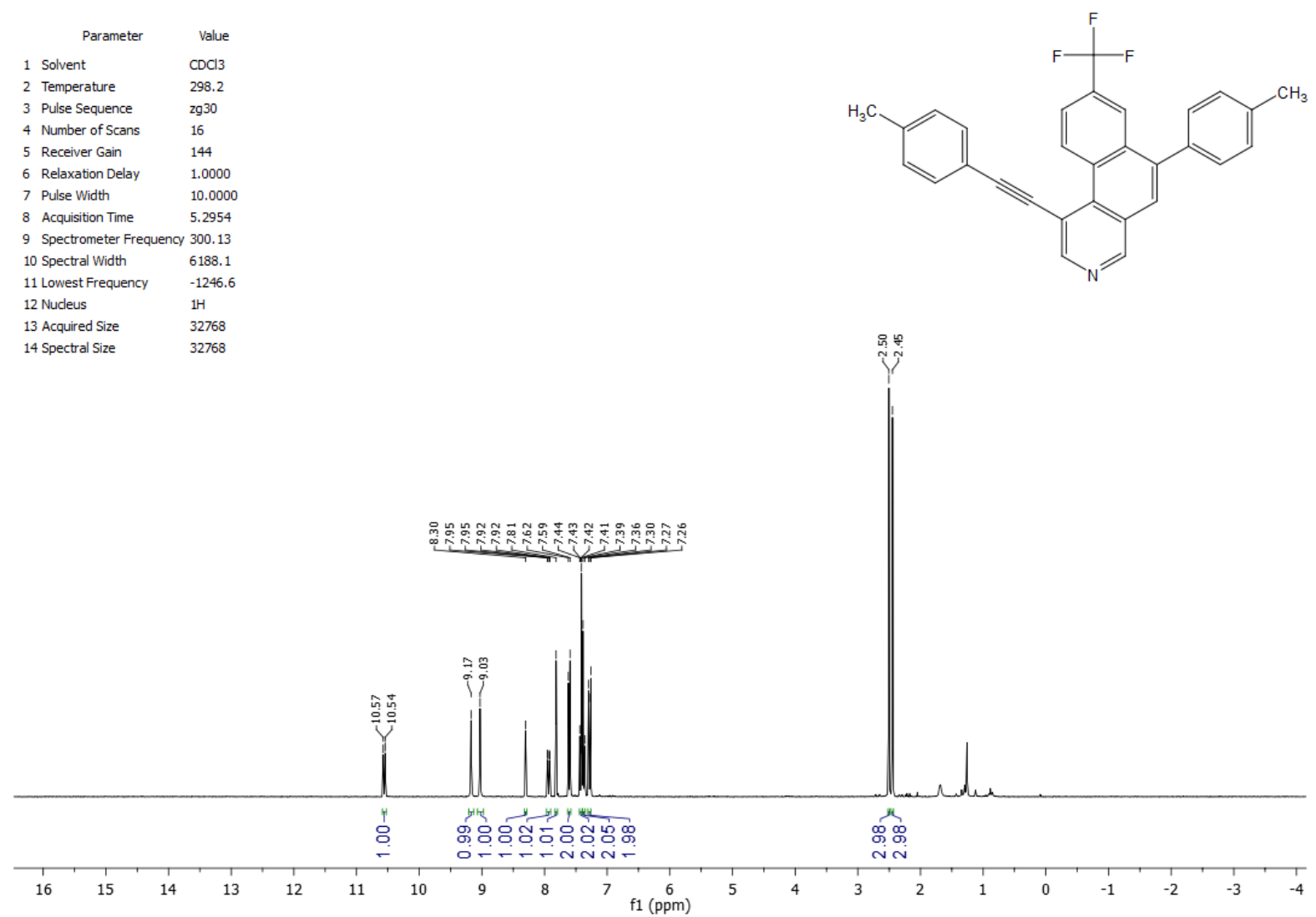

\begin{tabular}{|c|c|c|}
\hline & Parameter & Value \\
\hline & Solvent & $\mathrm{CDCl} 3$ \\
\hline & Temperature & 298.2 \\
\hline & Pulse Sequence & zgfhigan \\
\hline & Number of Scans & 64 \\
\hline & Receiver Gain & 2050 \\
\hline & Relaxation Delay & 1.0000 \\
\hline & Pulse Width & 10.0000 \\
\hline & Acquisition Time & 0.9787 \\
\hline & Spectrometer Frequency & 282.40 \\
\hline & Spectral Width & 66964.3 \\
\hline & Lowest Frequency & -61722 \\
\hline & Nucleus & $19 F$ \\
\hline & Acquired Size & 65536 \\
\hline & Spectral Size & 65536 \\
\hline
\end{tabular}

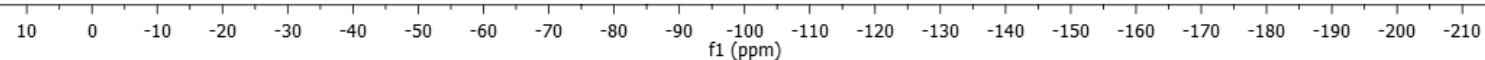




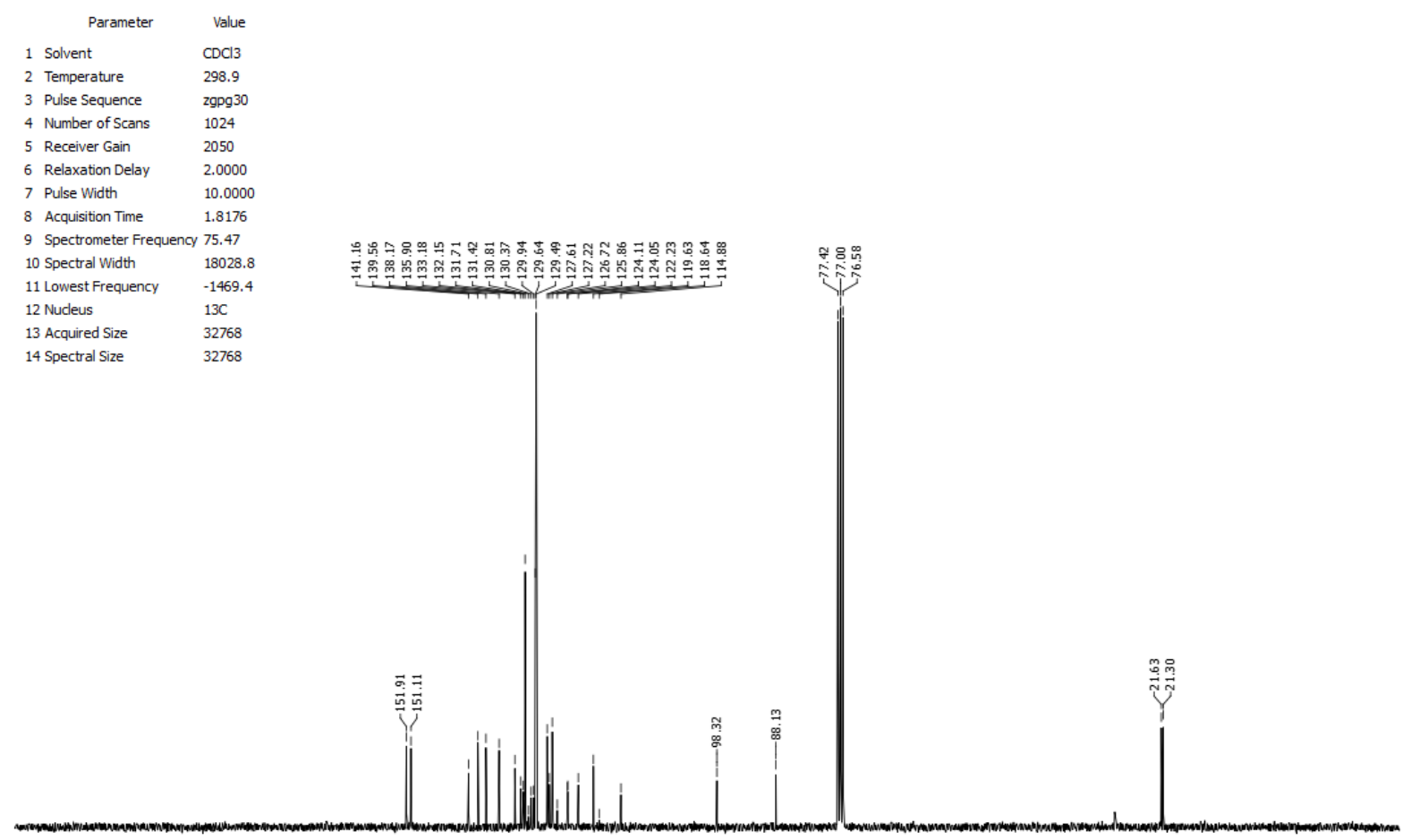

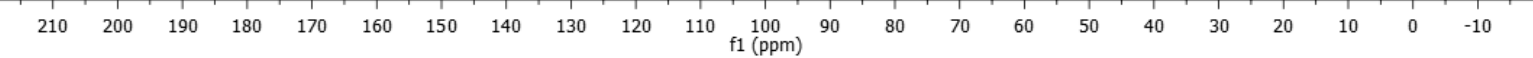


6-(4-fluorophenyl)-1-((4-fluorophenyl)ethynyl)benzo[f]isoquinoline $(4 \mathrm{~g})$

\begin{tabular}{|c|c|c|}
\hline & Parameter & Value \\
\hline & Solvent & $\mathrm{CDCl} 3$ \\
\hline 2 & Temperature & 298.2 \\
\hline 3 & Pulse Sequence & 2930 \\
\hline 4 & Number of Scans & 16 \\
\hline 5 & Receiver Gain & 72 \\
\hline 6 & Relaxation Delay & 1.0000 \\
\hline 7 & Pulse Width & 10.0000 \\
\hline 8 & Acquisition Time & 5.2954 \\
\hline & Spectrometer Frequency & 300.13 \\
\hline & Spectral Width & 6188.1 \\
\hline & Lowest Frequency & -1245.7 \\
\hline & Nucleus & $1 \mathrm{H}$ \\
\hline & Acquired Size & 32768 \\
\hline & Spectral Size & 32768 \\
\hline
\end{tabular}
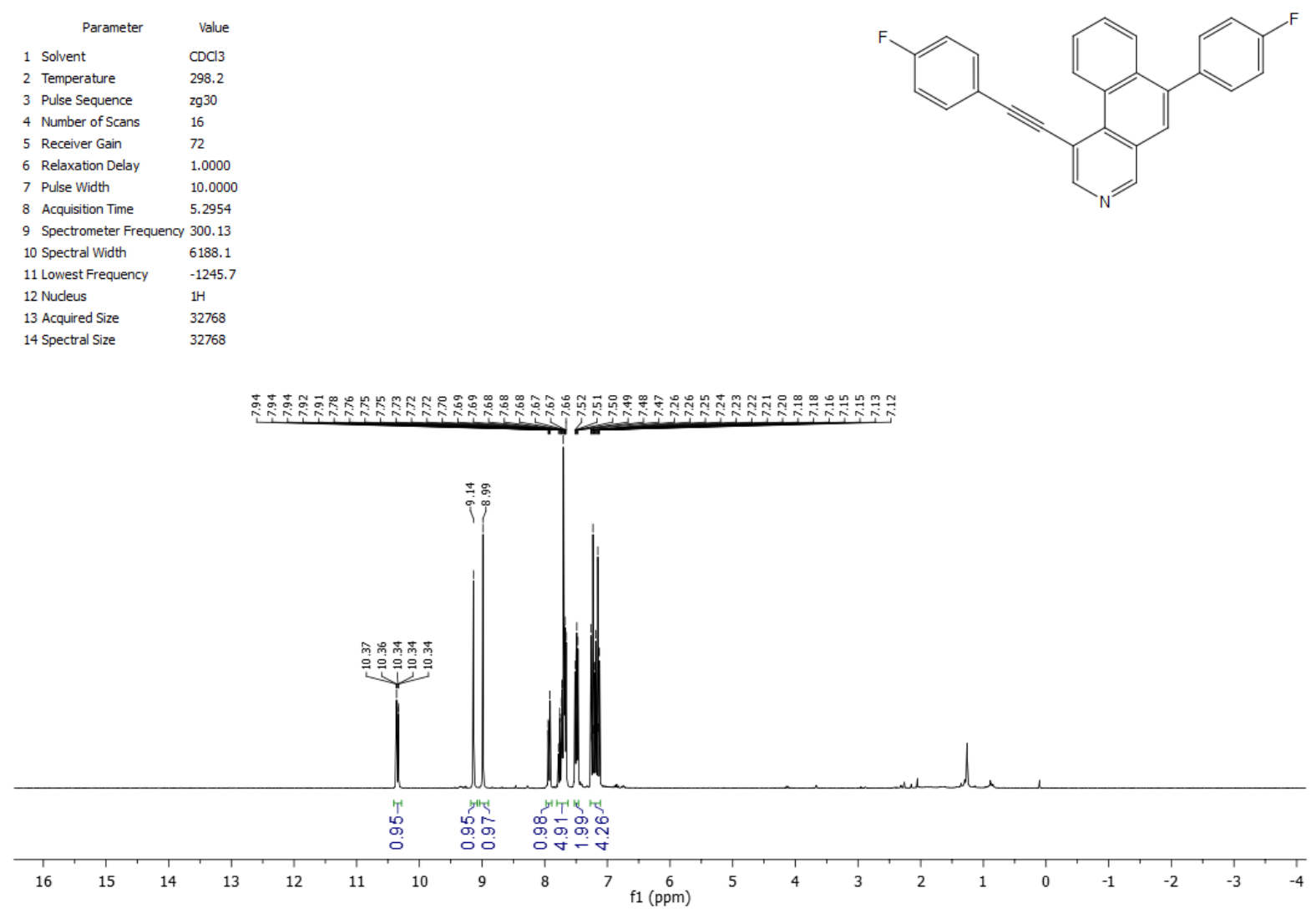

\begin{tabular}{lll} 
& \multicolumn{1}{c}{ Parameter } & \multicolumn{1}{c}{ Value } \\
1 Solvent & CDCl3 \\
2 Temperature & 298.2 \\
3 Pulse Sequence & zgfhigan \\
4 Number of Scans & 64 \\
5 & Receiver Gain & 2050 \\
6 & Relaxation Delay & 1.0000 \\
7 Pulse Width & 10.0000 \\
8 Acquisition Time & 0.9787 \\
9 Spectrometer Frequency 282.40 \\
10 Spectral Width & 66964.3 \\
11 Lowest Frequency & -61722.4 \\
12 Nucleus & $19 F$ \\
13 Acquired Size & 65536 \\
14 Spectral Size & 65536
\end{tabular}

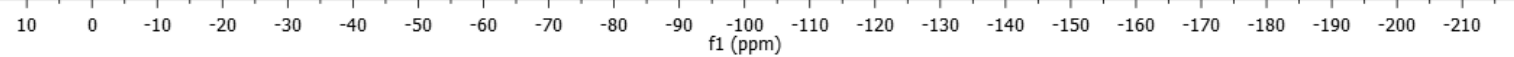




$$
\begin{aligned}
& \text { Parameter Value } \\
& 1 \text { Solvent } \mathrm{CDCl} 3 \\
& 2 \text { Temperature } 298.2 \\
& 3 \text { Pulse Sequence zgpg30 } \\
& 4 \text { Number of Scans } 1500 \\
& 5 \text { Receiver Gain } 2050 \\
& 6 \text { Relaxation Delay } 2.0000 \\
& 7 \text { Pulse Width } 10.0000 \\
& 8 \text { Acquisition Time } 1.8176 \\
& 10 \text { Spectral Width } \quad 18028 \text {. } \\
& 11 \text { Lowest Frequency } \quad-1471.6 \\
& 12 \text { Nucleus } \quad 13 \mathrm{C} \\
& 13 \text { Acquired Size } \quad 32768 \\
& 14 \text { Spectral Size } \quad 32768
\end{aligned}
$$

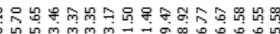

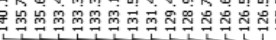

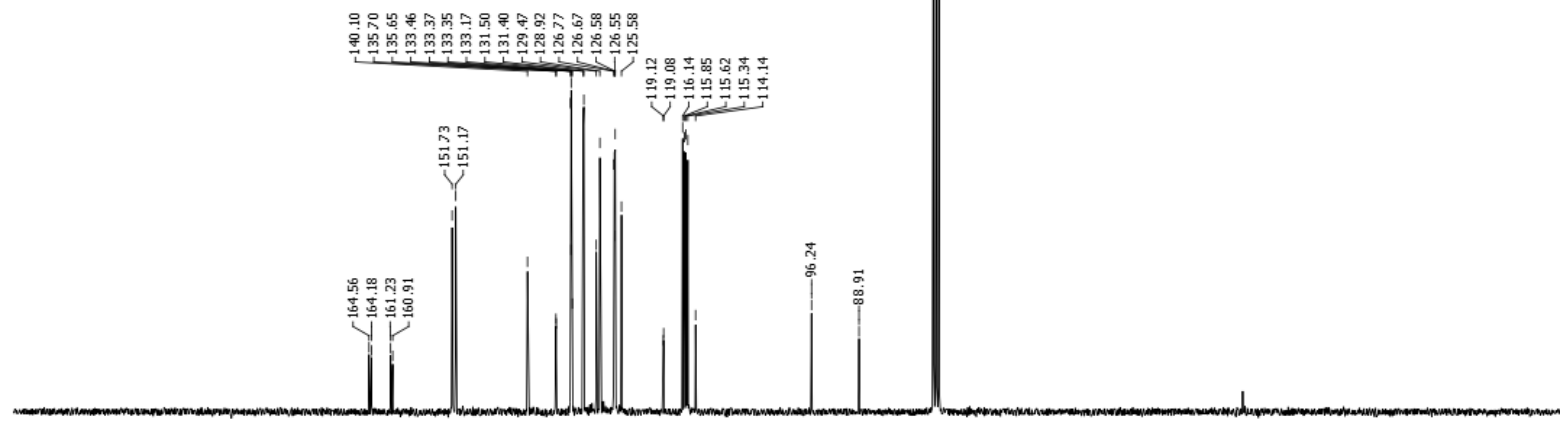

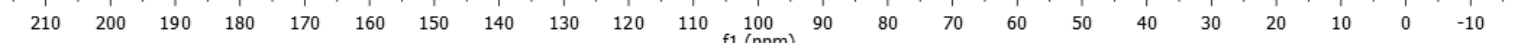



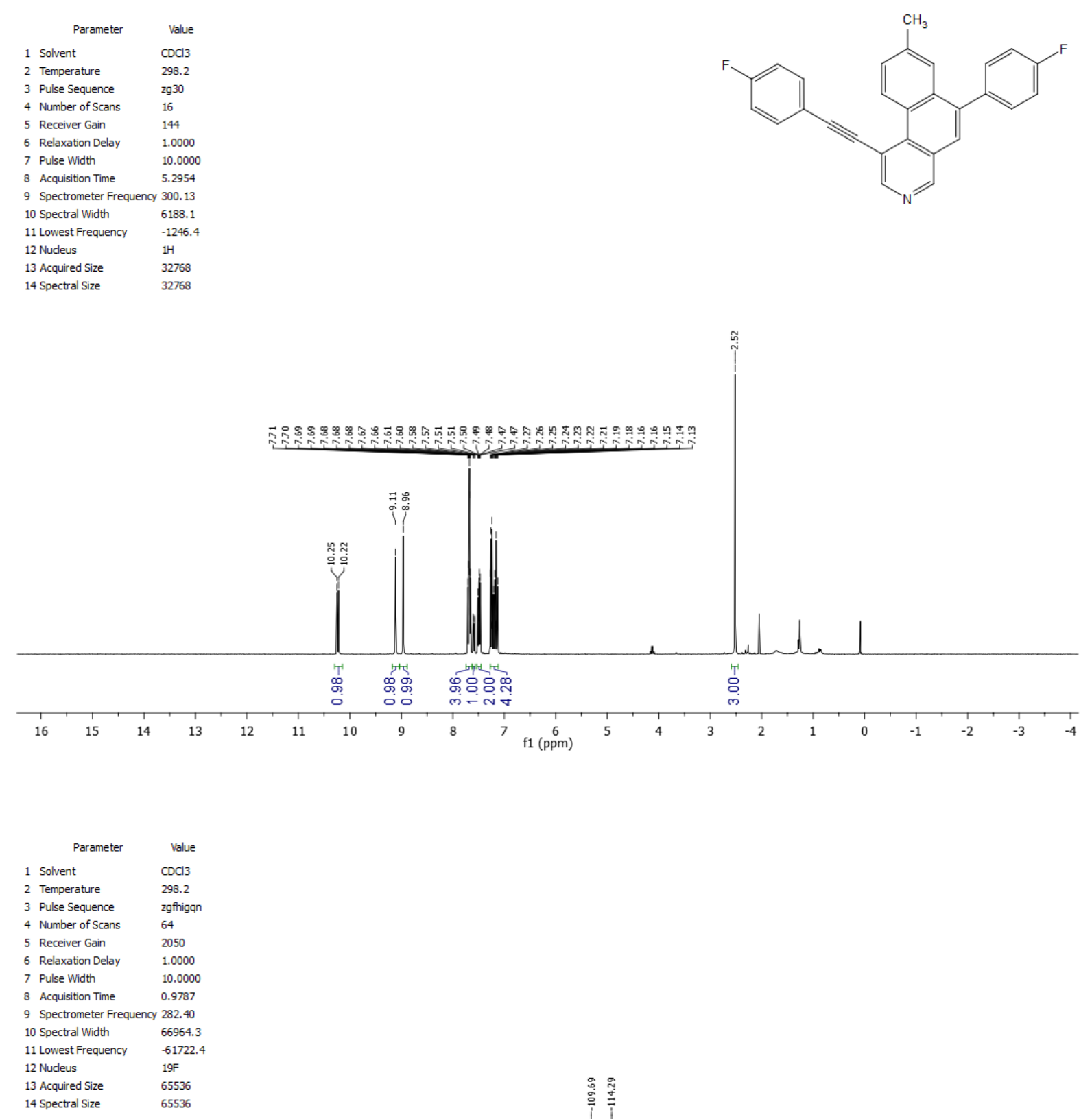

Value
CDCl3
98.2
gfhigan
4
2050
1.0000
0.0000
0.9787
282.40
66964.3
61722.4
$19 F$
65536
65536

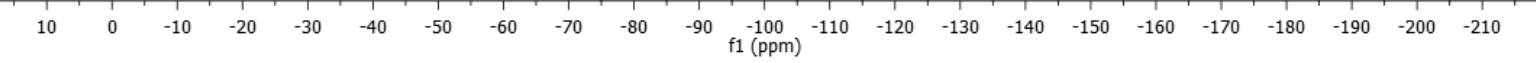




$$
\begin{array}{lll}
\multicolumn{1}{c}{\text { Parameter }} & \multicolumn{1}{c}{\text { Value }} \\
1 \text { Solvent } & \text { CDCl3 } \\
\text { 2 } & \text { Temperature } & 298.2 \\
3 \text { Pulse Sequence } & \text { 29pg } 30 \\
4 \text { Number of Scans } & 1024 \\
5 \text { Receiver Gain } & 2050 \\
6 \text { Relaxation Delay } & 2.0000 \\
7 \text { Pulse Width } & 10.0000 \\
8 \text { Acquisition Time } & 1.8176 \\
9 \text { Spectrometer Frequency } & 75.47 \\
\text { 10 Spectral Width } & 18028.8 \\
\text { 11 Lowest Frequency } & -1469.9 \\
\text { 12 Nucleus } & 13 \mathrm{C} \\
\text { 13 Acquired Size } & 32768 \\
\text { 14 Spectral Size } & 32768
\end{array}
$$

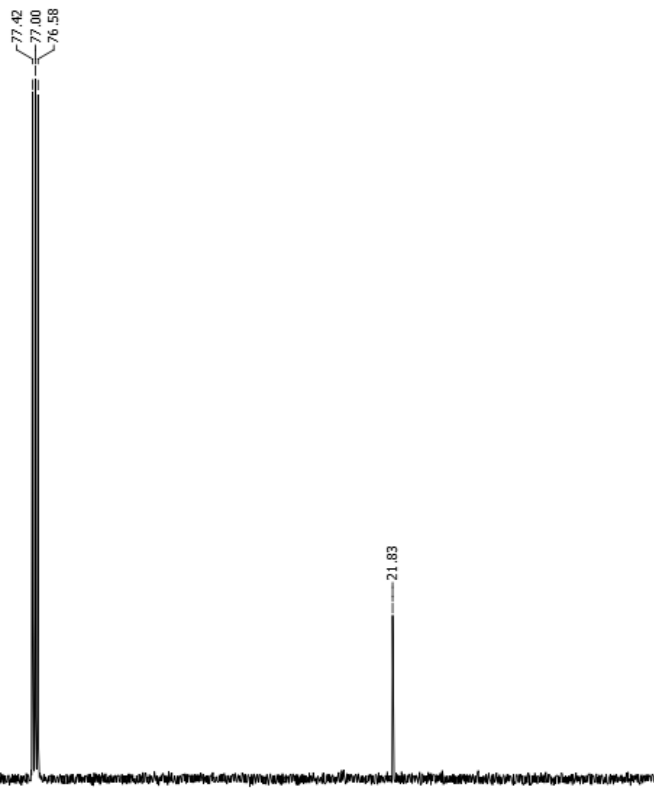

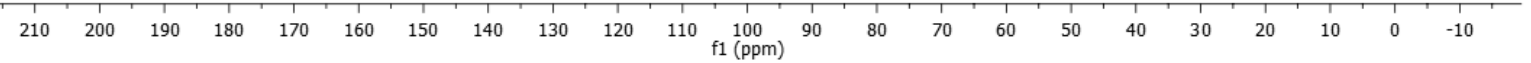


6-(4-(trifluoromethyl)phenyl)-1-((4-(trifluoromethyl)phenyl)ethynyl)benzo[ $f$ isoquinoline (4i)

\begin{tabular}{|c|c|c|}
\hline & Parameter & Value \\
\hline & Solvent & $\mathrm{CDCl} 3$ \\
\hline & Temperature & 298.1 \\
\hline & Pulse Sequence & 2930 \\
\hline & Number of Scans & 16 \\
\hline 5 & Receiver Gain & 63 \\
\hline 6 & Relaxation Delay & 1.0000 \\
\hline & Pulse Width & 8.0000 \\
\hline 8 & Acquisition Time & 3.2768 \\
\hline 9 & Spectrometer Frequency & 500.13 \\
\hline & Spectral Width & 10000.0 \\
\hline & Lowest Frequency & -1924.2 \\
\hline & Nudeus & $1 \mathrm{H}$ \\
\hline & Acquired Size & 32768 \\
\hline & Spectral Size & 65536 \\
\hline
\end{tabular}
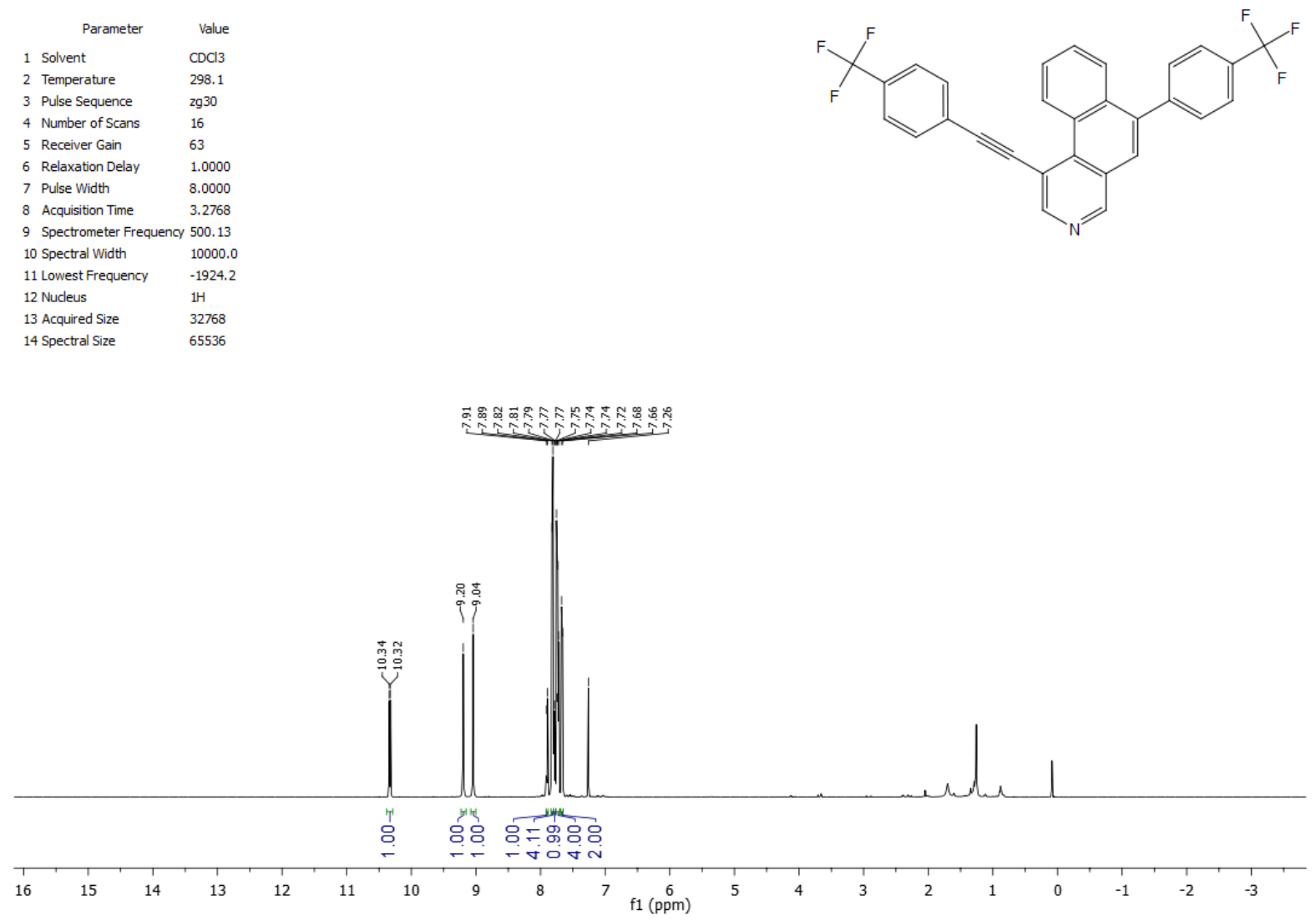

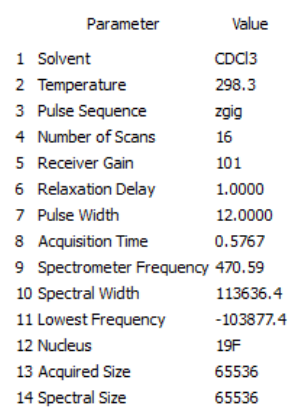




$$
\begin{array}{lll}
\multicolumn{1}{c}{\text { Parameter }} & \multicolumn{1}{c}{\text { Value }} \\
1 \text { Solvent } & \text { CDCl3 } \\
2 \text { Temperature } & 298.2 \\
3 \text { Pulse Sequence } & \text { 29pg } 30 \\
4 \text { Number of Scans } & 1024 \\
5 \text { Receiver Gain } & 101 \\
6 \text { Relaxation Delay } & 2.0000 \\
7 \text { Pulse Width } & 10.0000 \\
8 \text { Acquisition Time } & 1.0879 \\
9 \text { Spectrometer Frequency } & 125.76 \\
10 \text { Spectral Width } & 30120.5 \\
\text { 11 Lowest Frequency } & -2488.0 \\
\text { 12 Nucleus } & 13 \mathrm{C} \\
\text { 13 Acquired Size } & 32768 \\
\text { 14 Spectral Size } & 32768
\end{array}
$$

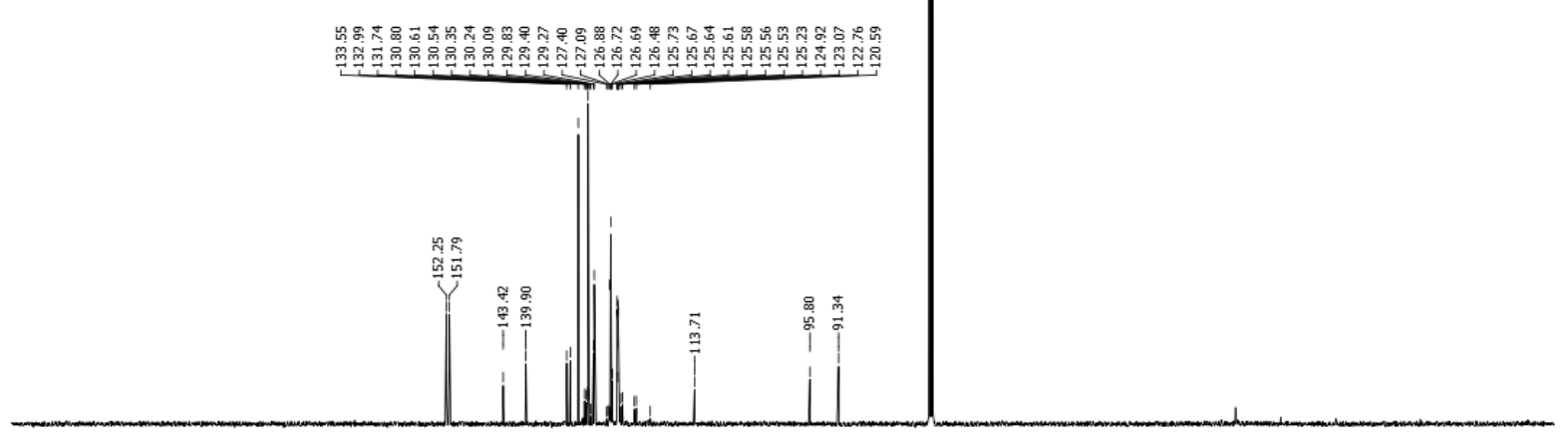

$\begin{array}{llllllllllllllllllllllllll}210 & 200 & 190 & 180 & 170 & 160 & 150 & 140 & 130 & 120 & 110 & 100 & 90 & 80 & 70 & 60 & 50 & 40 & 30 & 20 & 10 & 0 & -10\end{array}$ 
8-methyl-6-(4-(trifluoromethyl)phenyl)-1-((4-

(trifluoromethyl)phenyl)ethynyl)benzo[f]isoquinoline (4j)

\begin{tabular}{lll} 
& \multicolumn{1}{c}{ Parameter } & \multicolumn{1}{c}{ Value } \\
1 Solvent & CDCl3 \\
2 Temperature & 298.2 \\
3 Pulse Sequence & $\mathrm{zg} 30$ \\
4 Number of Scans & 16 \\
5 & Receiver Gain & 144 \\
6 Relaxation Delay & 1.0000 \\
7 Pulse Width & 10.0000 \\
8 Acquisition Time & 5.2954 \\
9 & Spectrometer Frequency & 300.13 \\
10 & Spectral Width & 6188.1 \\
11 Lowest Frequency & -1246.3 \\
12 Nucleus & $1 \mathrm{H}$ \\
13 Acquired Size & 32768 \\
14 Spectral Size & 32768
\end{tabular}
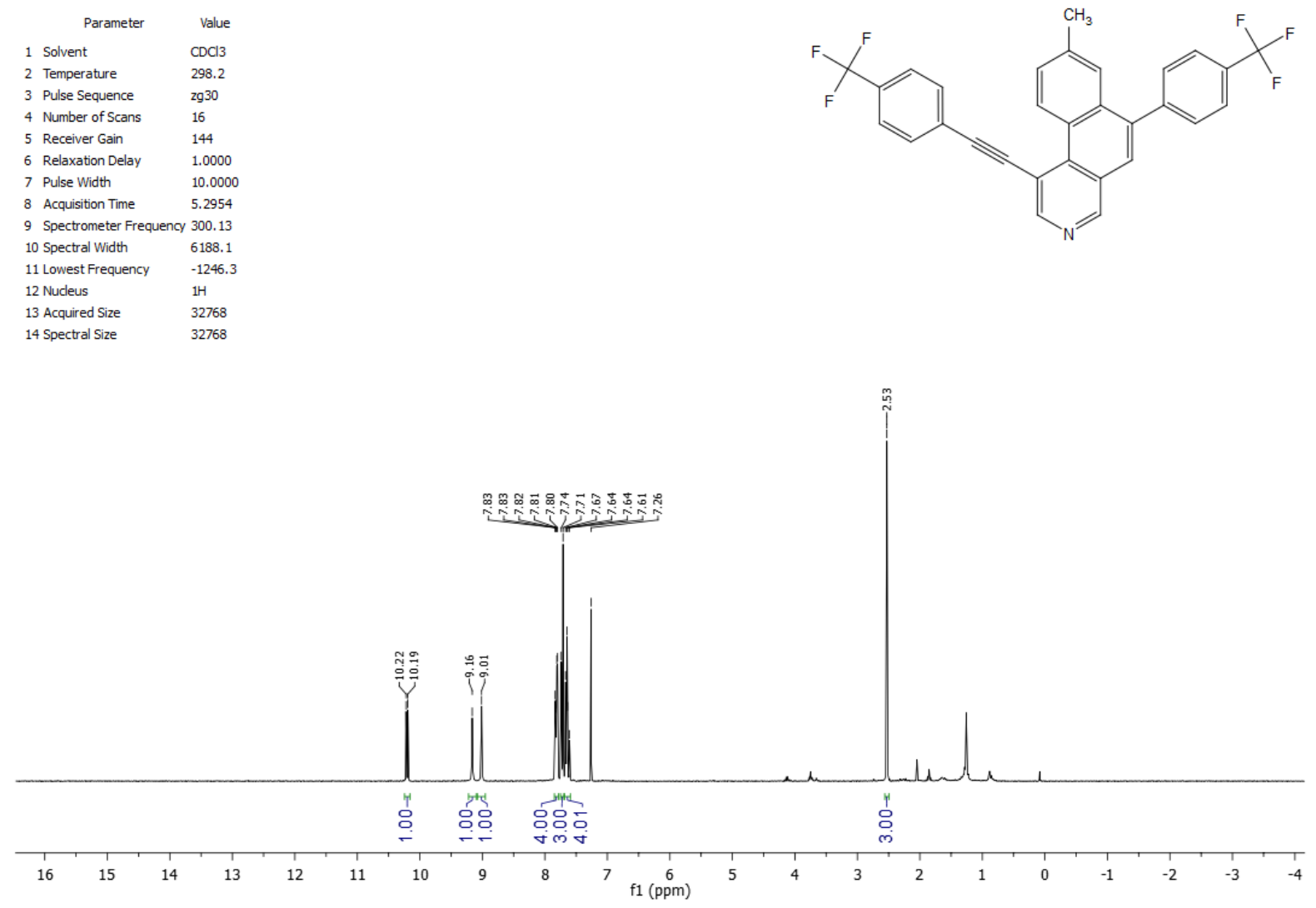

\begin{tabular}{lll}
\multicolumn{1}{c}{ Parameter } & \multicolumn{1}{c}{ Value } \\
1 Solvent & CDCl3 \\
2 2emperature & 298.2 \\
3 Pulse Sequence & 2gfhigqn \\
4 Number of Scans & 64 \\
5 & Receiver Gain & 2050 \\
6 Relaxation Delay & 1.0000 \\
7 Pulse Width & 10.0000 \\
8 Acquisition Time & 0.9787 \\
9 Spectrometer Frequency & 282.40 \\
10 Spectral Width & 66964.3 \\
11 Lowest Frequency & -61722.4 \\
12 Nudeus & $19 F$ \\
13 Acquired Size & 65536 \\
14 Spectral Size & 65536
\end{tabular}




$$
\begin{aligned}
& \text { Parameter Value } \\
& 1 \text { Solvent } \mathrm{CDCl} 3 \\
& 2 \text { Temperature } 298.2 \\
& 3 \text { Pulse Sequence } 2 \text { gpg } 30 \\
& 4 \text { Number of Scans } 1024 \\
& 5 \text { Receiver Gain } 2050 \\
& 6 \text { Relaxation Delay } \quad 2.0000 \\
& 7 \text { Pulse Width } 10.000 \\
& 8 \text { Acquisition Time } 1.8176 \\
& 10 \text { Spectral Width } \quad 18028.8 \\
& 11 \text { Lowest Frequency } \quad-1468.1 \\
& 12 \text { Nucleus } \quad 13 \mathrm{C} \\
& 13 \text { Acquired Size } \quad 32768 \\
& 14 \text { Spectral Size } \quad 32768
\end{aligned}
$$
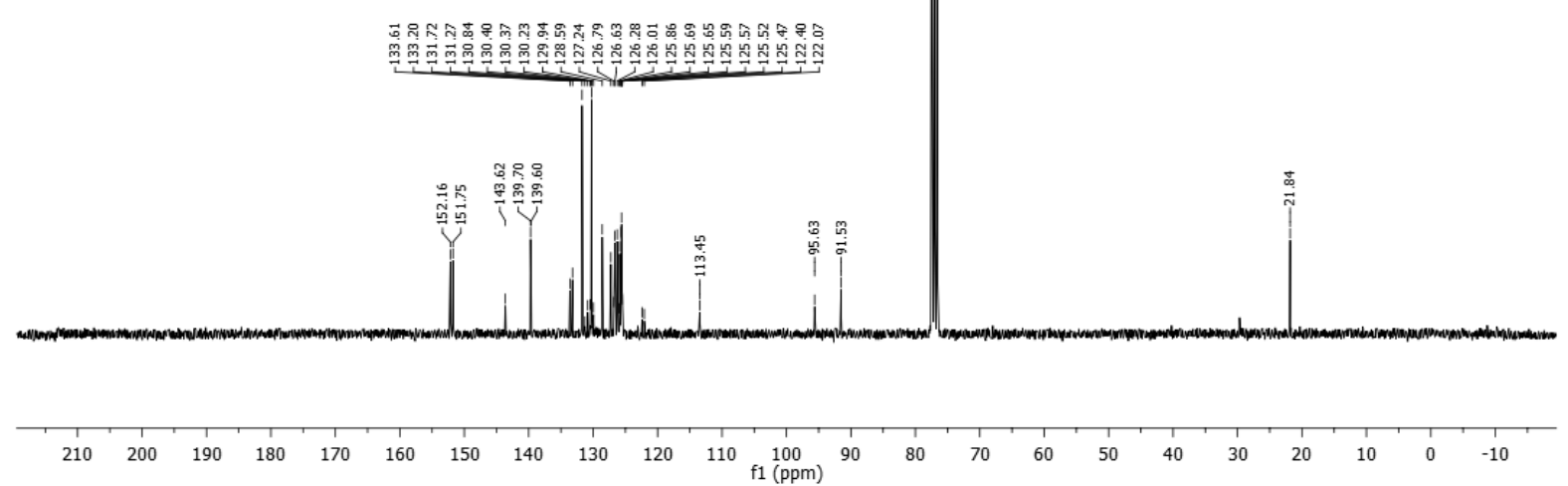


\begin{tabular}{lll}
\multicolumn{1}{c}{ Parameter } & \multicolumn{1}{c}{ Value } \\
1 Solvent & CDCl3 \\
2 2 & Temperature & 298.2 \\
3 Pulse Sequence & 2930 \\
4 Number of Scans & 16 \\
5 Receiver Gain & 144 \\
6 Relaxation Delay & 1.0000 \\
7 Pulse Width & 10.0000 \\
8 Acquisition Time & 5.2954 \\
9 Spectrometer Frequency & 300.13 \\
10 Spectral Width & 6188.1 \\
11 Lowest Frequency & -1246.3 \\
12 Nucleus & $1 \mathrm{H}$ \\
13 Acquired Size & 32768 \\
14 Spectral Size & 32768
\end{tabular}
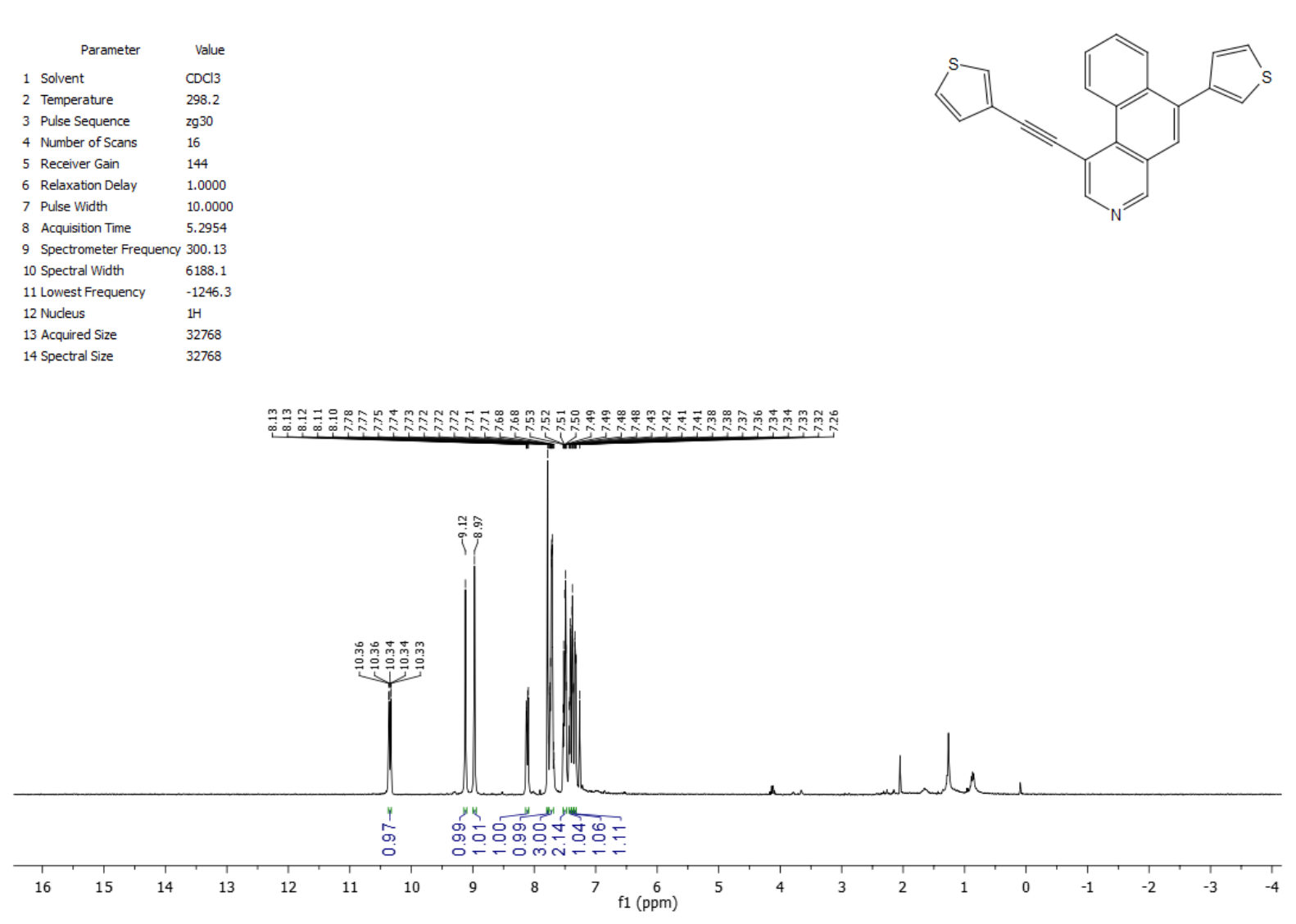

$$
\begin{array}{lll}
\multicolumn{1}{c}{\text { Parameter }} & \multicolumn{1}{c}{\text { Value }} \\
1 \text { Solvent } & \text { CDCl3 } \\
2 \text { 2emperature } & 298.2 \\
3 \text { Pulse Sequence } & 29 p g 30 \\
4 \text { Number of Scans } & 1024 \\
5 \text { Receiver Gain } & 2050 \\
6 \text { Relaxation Delay } & 2.0000 \\
7 \text { Pulse Width } & 10.0000 \\
8 \text { Acquisition Time } & 1.8176 \\
9 \text { Spectrometer Frequency } 75.47 \\
\text { 10 Spectral Width } & 18028.8 \\
\text { 11 Lowest Frequency } & -1472.0 \\
\text { 12 Nucleus } & 13 \mathrm{C} \\
\text { 13 Acquired Size } & 32768 \\
\text { 14 Spectral Size } & 32768
\end{array}
$$


5,9-diphenylnaphtho[2,1,8-def]isoquinoline (5a)

\begin{tabular}{lll}
\multicolumn{1}{c}{ Parameter } & \multicolumn{1}{c}{ Value } \\
1 Solvent & $\mathrm{CDCl} 3$ \\
2 & Temperature & 298.1 \\
3 Pulse Sequence & $\mathrm{zg} 30$ \\
4 Number of Scans & 16 \\
5 Receiver Gain & 63 \\
6 Relaxation Delay & 1.0000 \\
7 Pulse Width & 8.0000 \\
8 Acquisition Time & 3.2768 \\
9 Spectrometer Frequency & 500.13 \\
10 Spectral Width & 10000.0 \\
11 Lowest Frequency & -1924.1 \\
12 Nucleus & $1 H$ \\
13 Acquired Size & 32768 \\
14 Spectral Size & 65536
\end{tabular}
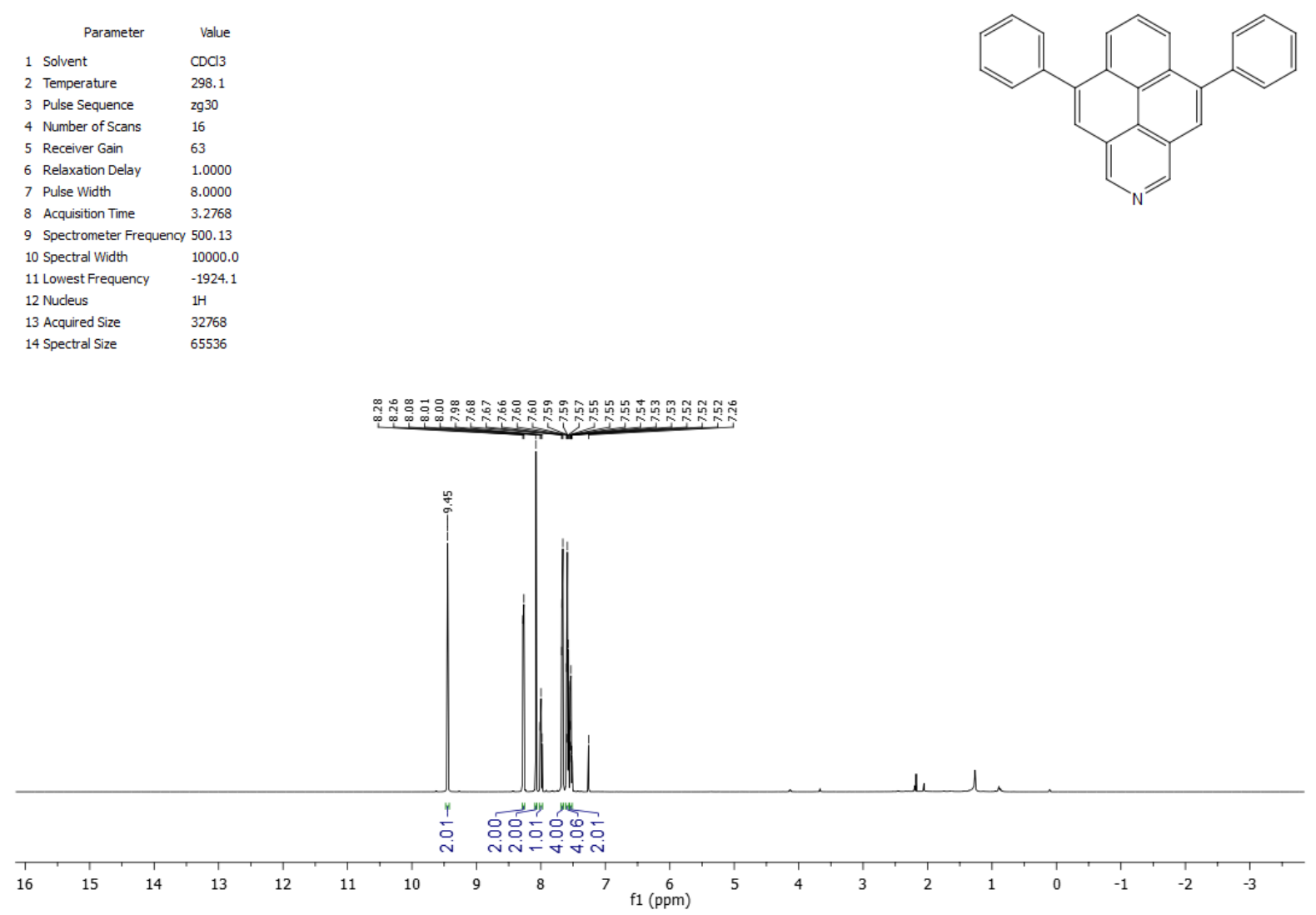

\begin{tabular}{lll}
\multicolumn{1}{c}{ Parameter } & \multicolumn{1}{c}{ Value } \\
1 Solvent & CDCl3 \\
2 2 & Pemperature & 298.2 \\
3 Pulse Sequence & $29 p 930$ \\
4 Number of Scans & 1024 \\
5 Receiver Gain & 101 \\
6 Relaxation Delay & 2.0000 \\
7 Pulse Width & 10.0000 \\
8 Acquisition Time & 1.0879 \\
9 Spectrometer Frequency 125.76 \\
10 Spectral Width & 30120.5 \\
11 Lowest Frequency & -2492.3 \\
12 Nucleus & $13 \mathrm{C}$ \\
13 Acquired Size & 32768 \\
14 Spectral Size & 32768
\end{tabular}
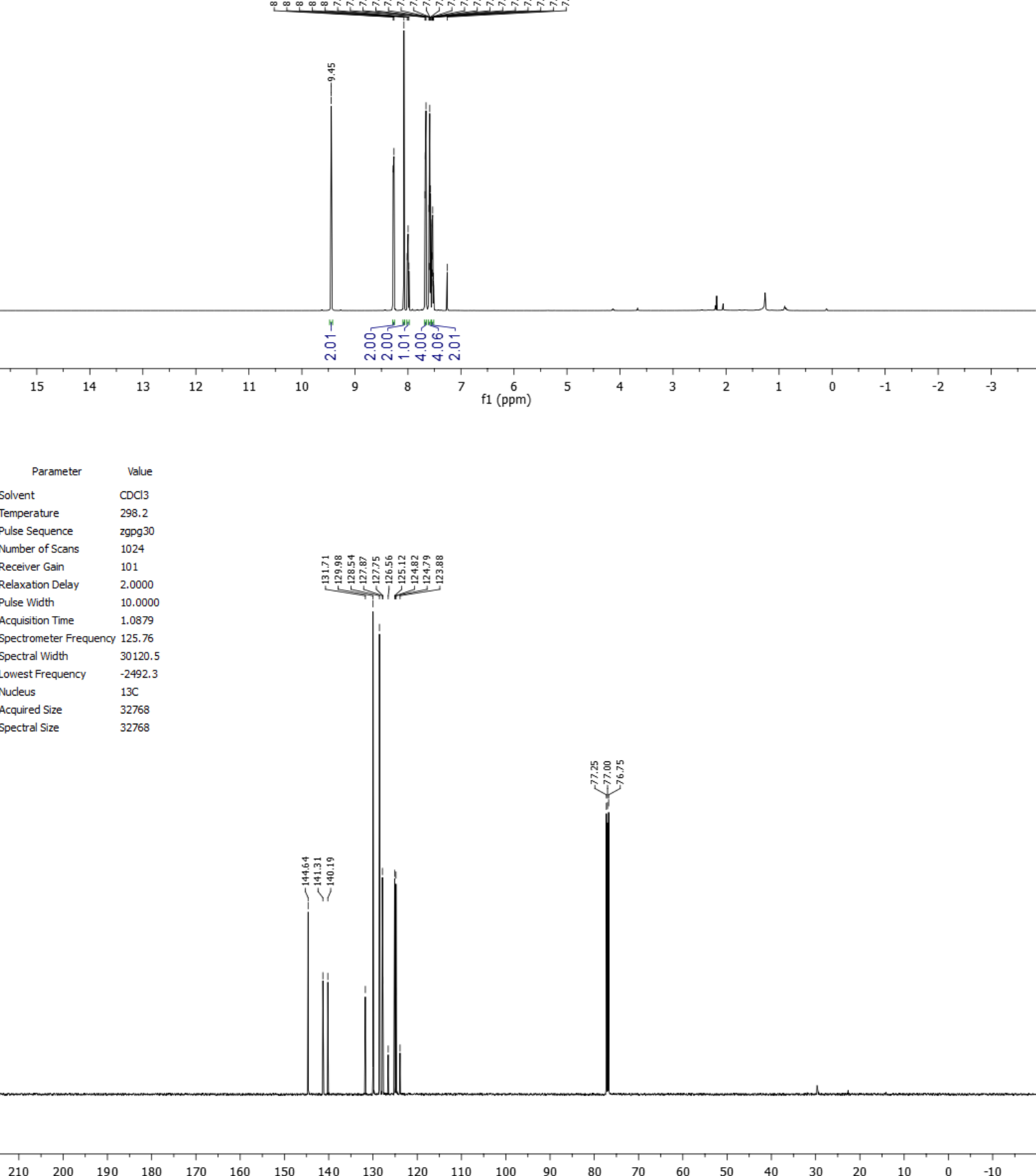

$\begin{array}{lllllllllll}210 & 200 & 190 & 180 & 170 & 160 & 150 & 140 & 130 & 120 & 110 \\ \mathrm{f} 1(\mathrm{ppm}) & 100\end{array}$ 
7-methyl-5,9-diphenylnaphtho[2,1,8-def]isoquinoline (5b)

\begin{tabular}{|c|c|c|}
\hline & Parameter & Valu \\
\hline & Solvent & $\mathrm{CDCl}$ \\
\hline & Temperature & 298.1 \\
\hline & Pulse Sequence & 2930 \\
\hline & Number of Scans & 16 \\
\hline & Receiver Gain & 63 \\
\hline & Relaxation Delay & 1.000 \\
\hline & Pulse Width & 8.00 \\
\hline & Acquisition Time & 3.2768 \\
\hline & Spectrometer Frequency & 500.1 \\
\hline & Spectral Width & 1000 \\
\hline & Lowest Frequency & -1924 \\
\hline & Nucleus & $1 \mathrm{H}$ \\
\hline & Acquired $S$ & 327 \\
\hline & & \\
\hline
\end{tabular}
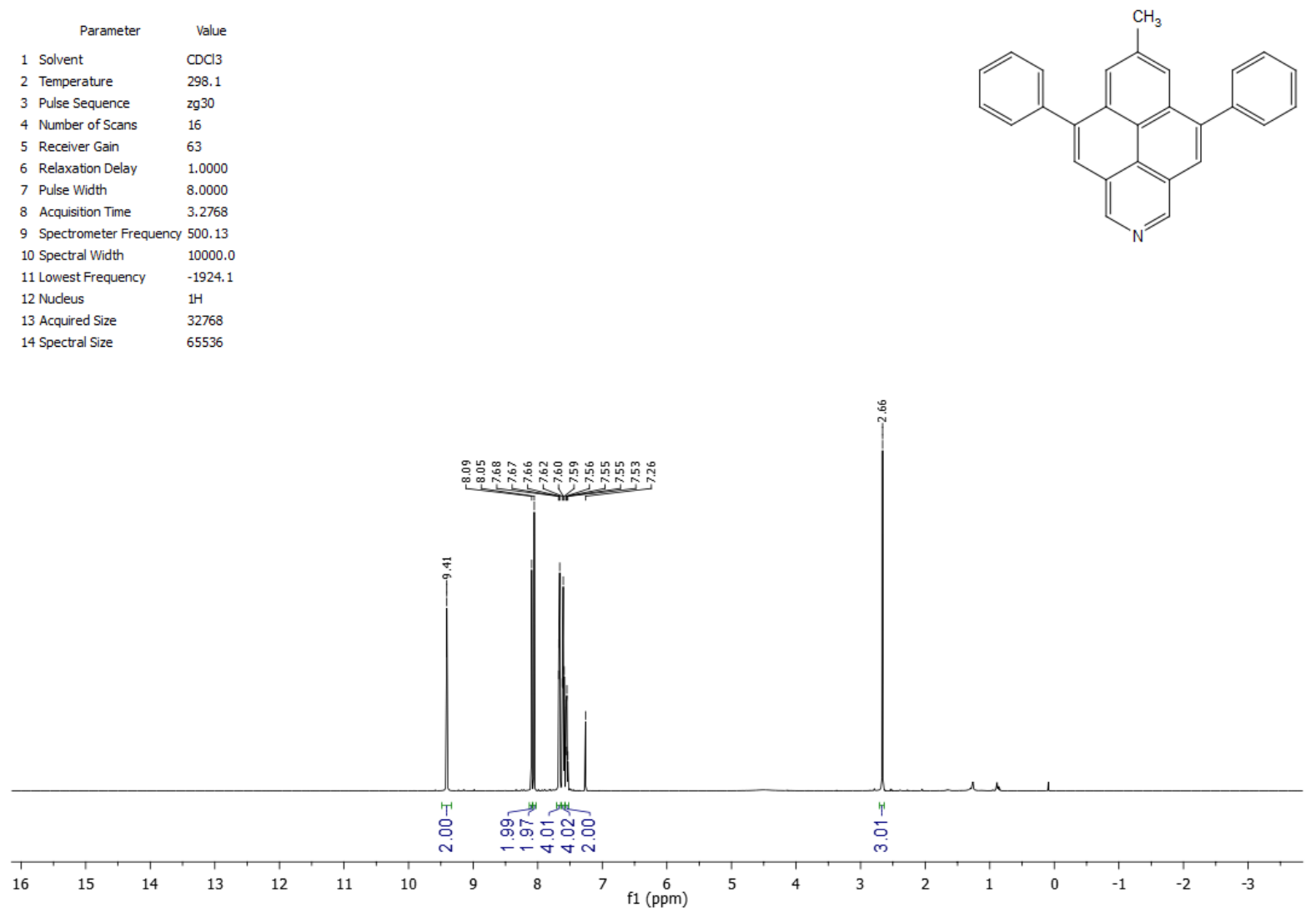

\begin{tabular}{|c|c|c|}
\hline & Parameter & Value \\
\hline & Solvent & $\mathrm{CDCl} 3$ \\
\hline & Temperature & 298.2 \\
\hline & Pulse Sequence & zgpg 30 \\
\hline & Number of Scans & 1024 \\
\hline & Receiver Gain & 101 \\
\hline & Relaxation Delay & 2.0000 \\
\hline & Pulse Width & 10.0000 \\
\hline & Acquisition Time & 1.0879 \\
\hline & Spectrometer Frequency & 125.76 \\
\hline & Spectral Width & 30120.5 \\
\hline & Lowest Frequency & -2491.0 \\
\hline & Nucleus & $13 \mathrm{C}$ \\
\hline & Acquired Size & 32768 \\
\hline & Spectral Size & 32768 \\
\hline
\end{tabular}
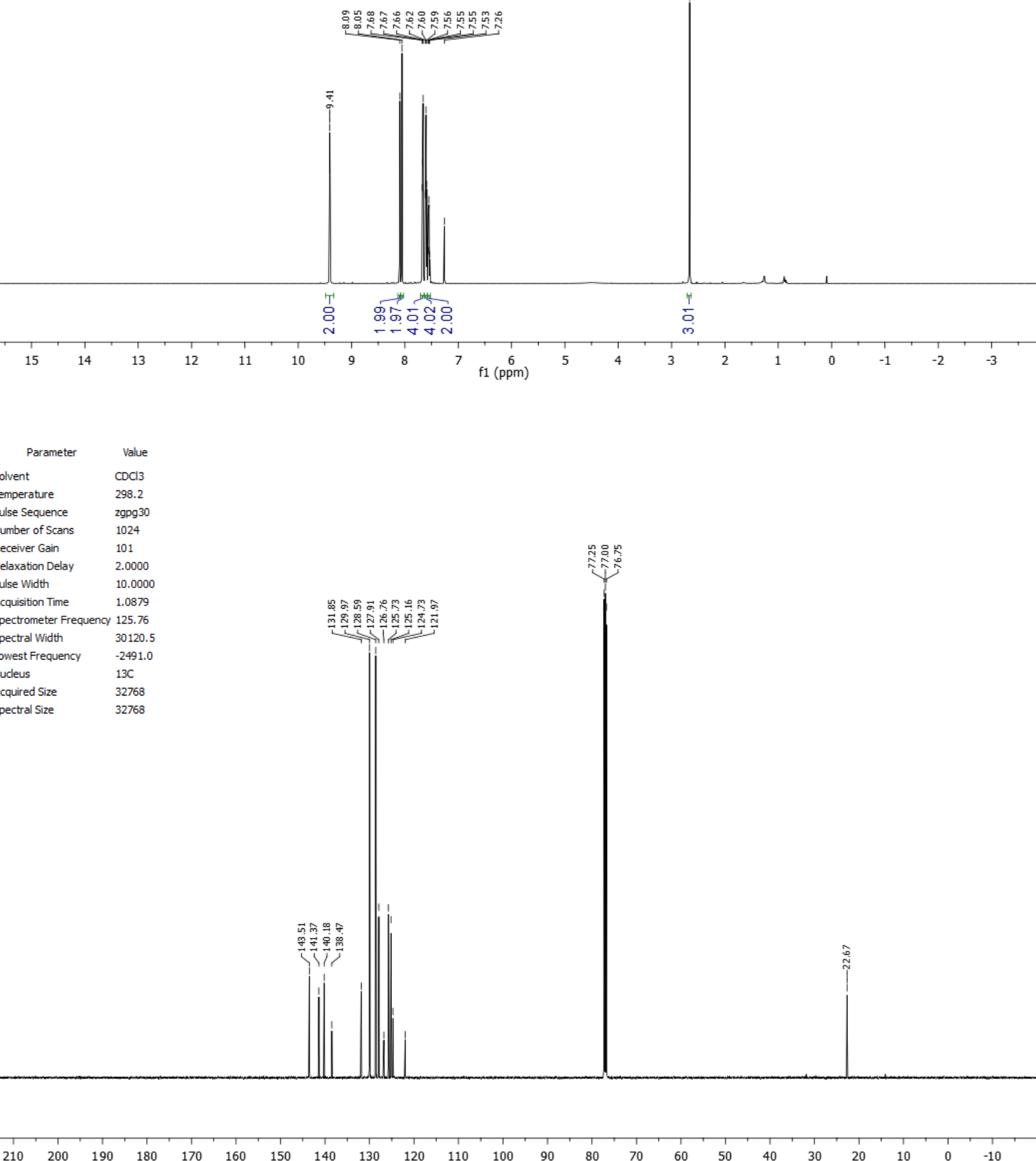

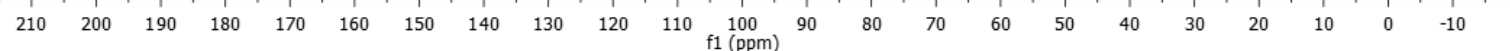


7-methoxy-5,9-diphenylnaphtho[2,1,8-def]isoquinoline (5c)

\begin{tabular}{lll}
\multicolumn{1}{c}{ Parameter } & \multicolumn{1}{c}{ Value } \\
1 Solvent & CDCl3 \\
2 Temperature & 298.2 \\
3 & Pulse Sequence & 2930 \\
4 Number of Scans & 16 \\
5 S & Receiver Gain & 228 \\
6 6elaxation Delay & 2.0000 \\
7 Pulse Width & 10.0000 \\
8 & Acquisition Time & 5.2954 \\
9 & Spectrometer Frequency 300.13 \\
10 & Spectral Width & 6188.1 \\
11 Lowest Frequency & -1246.3 \\
12 Nucleus & $1 \mathrm{H}$ \\
13 Acquired Size & 32768 \\
14 Spectral Size & 32768
\end{tabular}
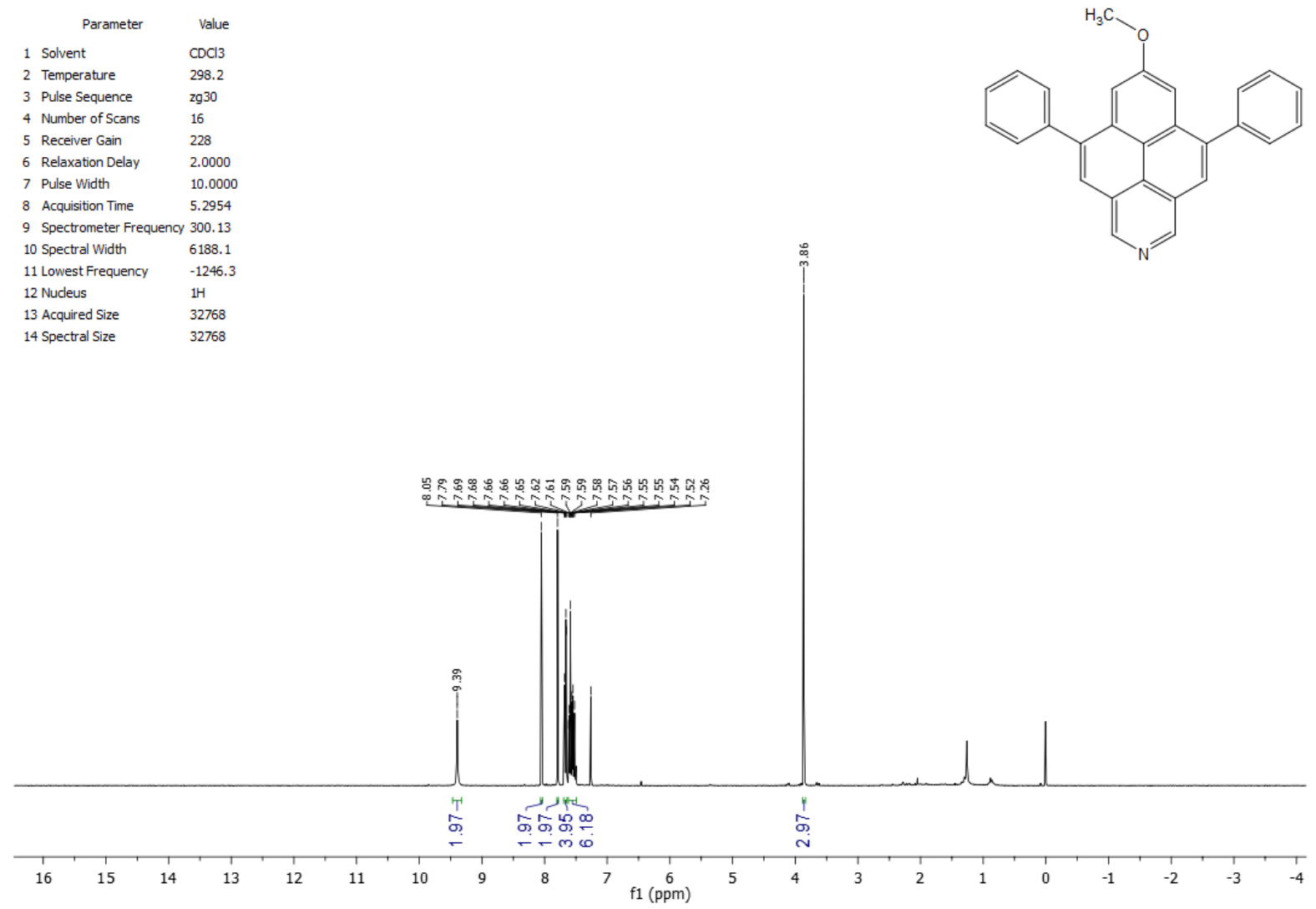

\begin{tabular}{|c|c|c|}
\hline & Parameter & Valu \\
\hline & Solvent & $\mathrm{CDCl}$ \\
\hline & Temperature & 298. \\
\hline & Pulse Sequence & \\
\hline & Number of Scans & 02 \\
\hline & Receiver Gain & 2050 \\
\hline & Relaxation Delay & 2.00 \\
\hline & Pulse Width & 10.0 \\
\hline & Acquisition Time & 1.81 \\
\hline & Spectrometer Frequency & 75.4 \\
\hline & Spectral Width & \\
\hline & Lowest Frequency & \\
\hline & Nucleus & \\
\hline & cquired Size & \\
\hline & pectral Size & \\
\hline
\end{tabular}

14 Spectral Size $\quad 32768$

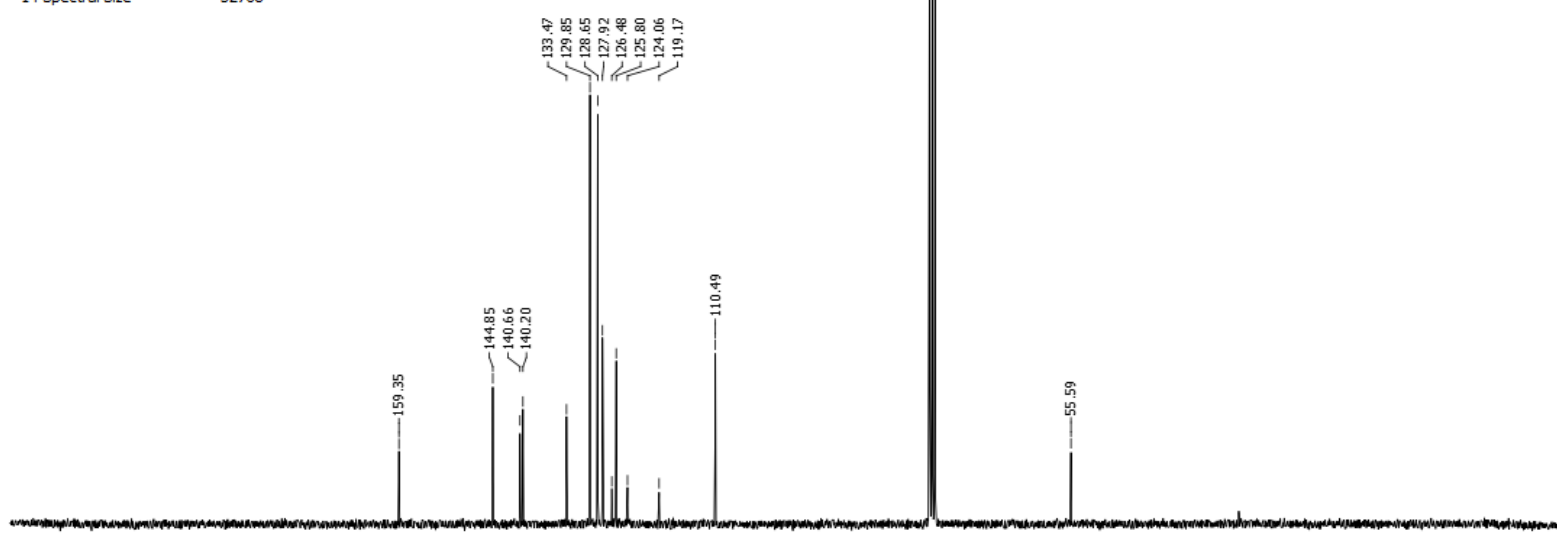

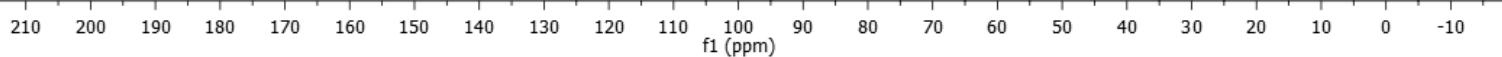


5,9-diphenyl-7-(trifluoromethyl)naphtho[2,1,8-def]isoquinoline (5d)

\begin{tabular}{lll}
\multicolumn{1}{c}{ Parameter } & \multicolumn{1}{c}{ Value } \\
1 Solvent & $\mathrm{CDCl3}$ \\
2 Temperature & 298.2 \\
3 Pulse Sequence & zg30 \\
4 Number of Scans & 16 \\
5 Receiver Gain & 144 \\
6 Relaxation Delay & 1.0000 \\
7 Pulse Width & 10.0000 \\
8 Acquisition Time & 5.2954 \\
9 Spectrometer Frequency & 300.13 \\
10 Spectral Width & 6188.1 \\
11 Lowest Frequency & -1246.4 \\
12 Nucleus & $1 \mathrm{H}$ \\
13 Acquired Size & 32768 \\
14 Spectral Size & 32768
\end{tabular}

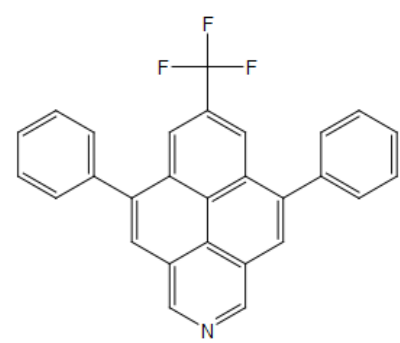

14 Spectral Size $\quad 32768$

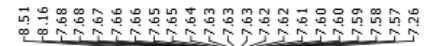

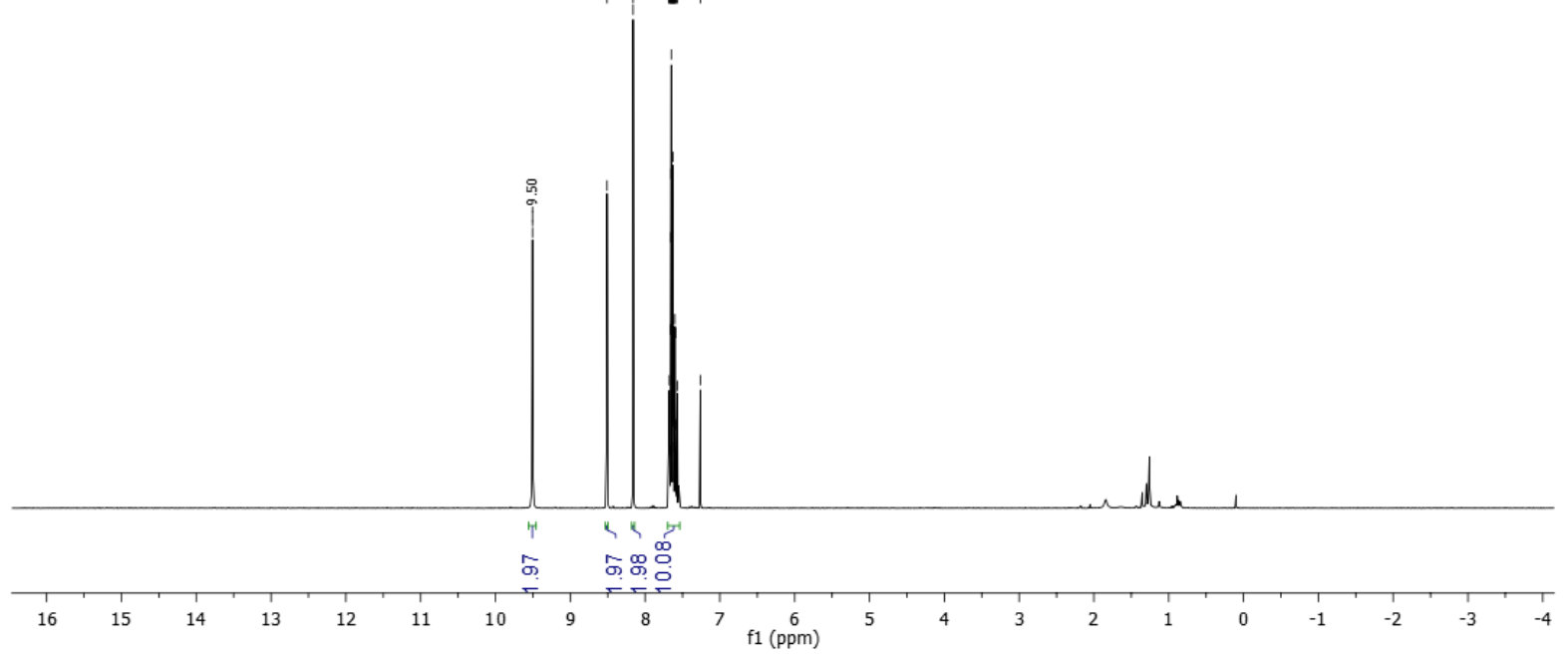

$$
\begin{aligned}
& \begin{array}{llr} 
& \text { Parameter } & \text { Value } \\
1 \text { Solvent } & \text { CDCl3 } \\
\text { 2 } & \text { Temperature } & 298.2
\end{array} \\
& \begin{array}{lll}
2 & \text { Temperature } & 298.2 \\
3 & \text { Pulse Sequence } & \text { zgfhigan }
\end{array} \\
& 4 \text { Number of Scans } 64 \\
& 5 \text { Receiver Gain } 2050 \\
& 6 \text { Relaxation Delay } 1.0000 \\
& 7 \text { Pulse Width } \quad 10.0000 \\
& 0.9787 \\
& 9 \text { Spectrometer Frequency } 282.40 \\
& 10 \text { Spectral Width } \quad 66964.3 \\
& 11 \text { Lowest Frequency } \quad-61722.4 \\
& 12 \text { Nucleus } \quad 19 F \\
& \begin{array}{ll}
13 \text { Acquired Size } & 65536 \\
14 \text { Spectral Size } & 65536
\end{array}
\end{aligned}
$$




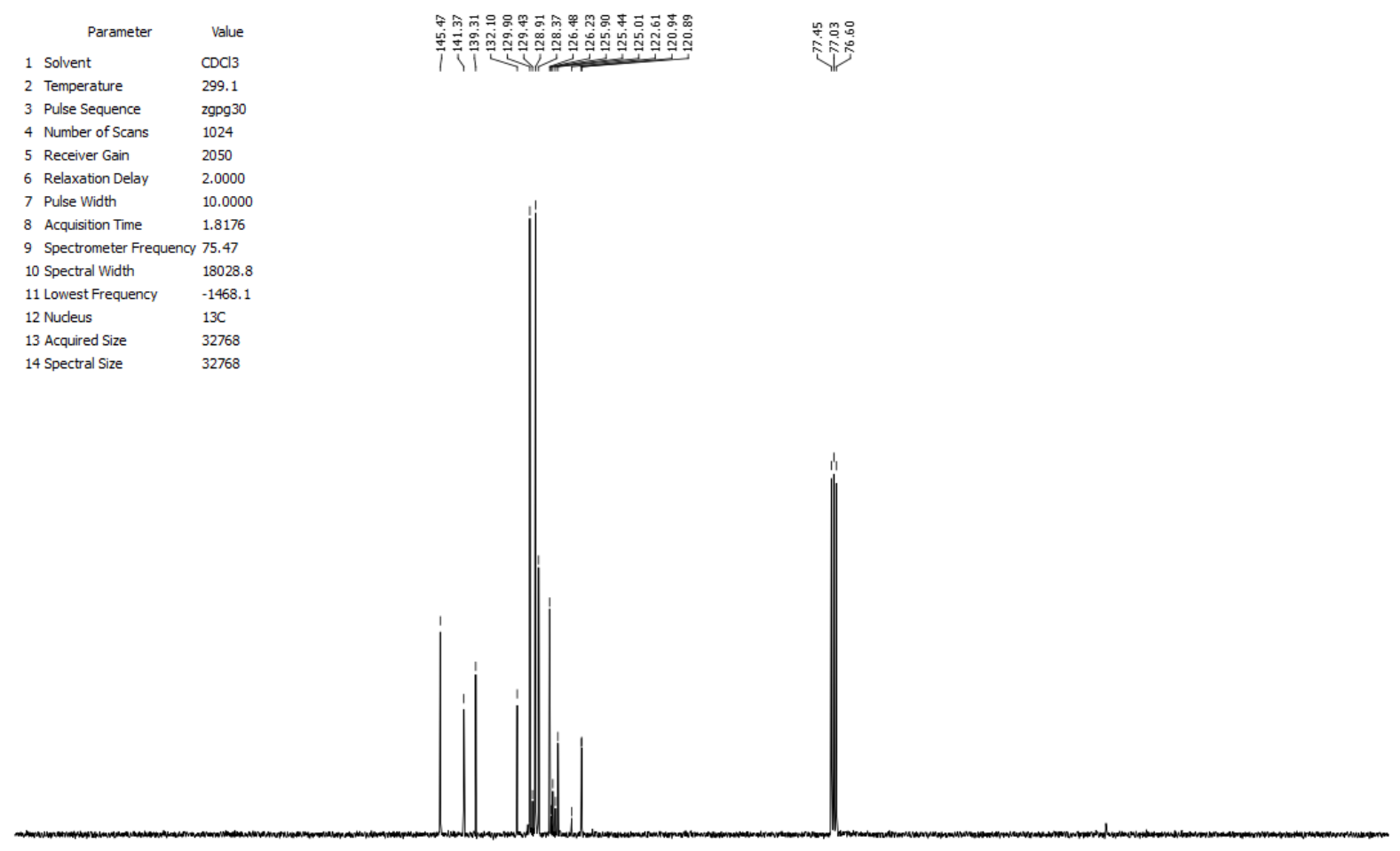

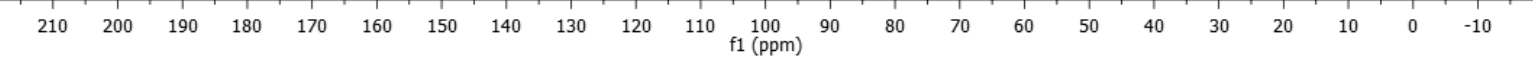




\section{5,9-di- $m$-tolylnaphtho[2,1,8-def]isoquinoline (5e)}

\begin{tabular}{|c|c|c|}
\hline & Parameter & Value \\
\hline & Solvent & $\mathrm{CDCl} 3$ \\
\hline & Temperature & 298.2 \\
\hline & Pulse Sequence & zg30 \\
\hline & Number of Scans & 16 \\
\hline & Receiver Gain & 43 \\
\hline & Relaxation Delay & 1.0000 \\
\hline & Pulse Width & 8.0000 \\
\hline & Acquisition Time & 3.2768 \\
\hline & Spectrometer Frequency & 500.13 \\
\hline & Spectral Width & 10000.0 \\
\hline & Lowest Frequency & -1924.2 \\
\hline & Nucleus & $1 \mathrm{H}$ \\
\hline & Acquired Size & 32768 \\
\hline & Spectral Size & 65536 \\
\hline
\end{tabular}
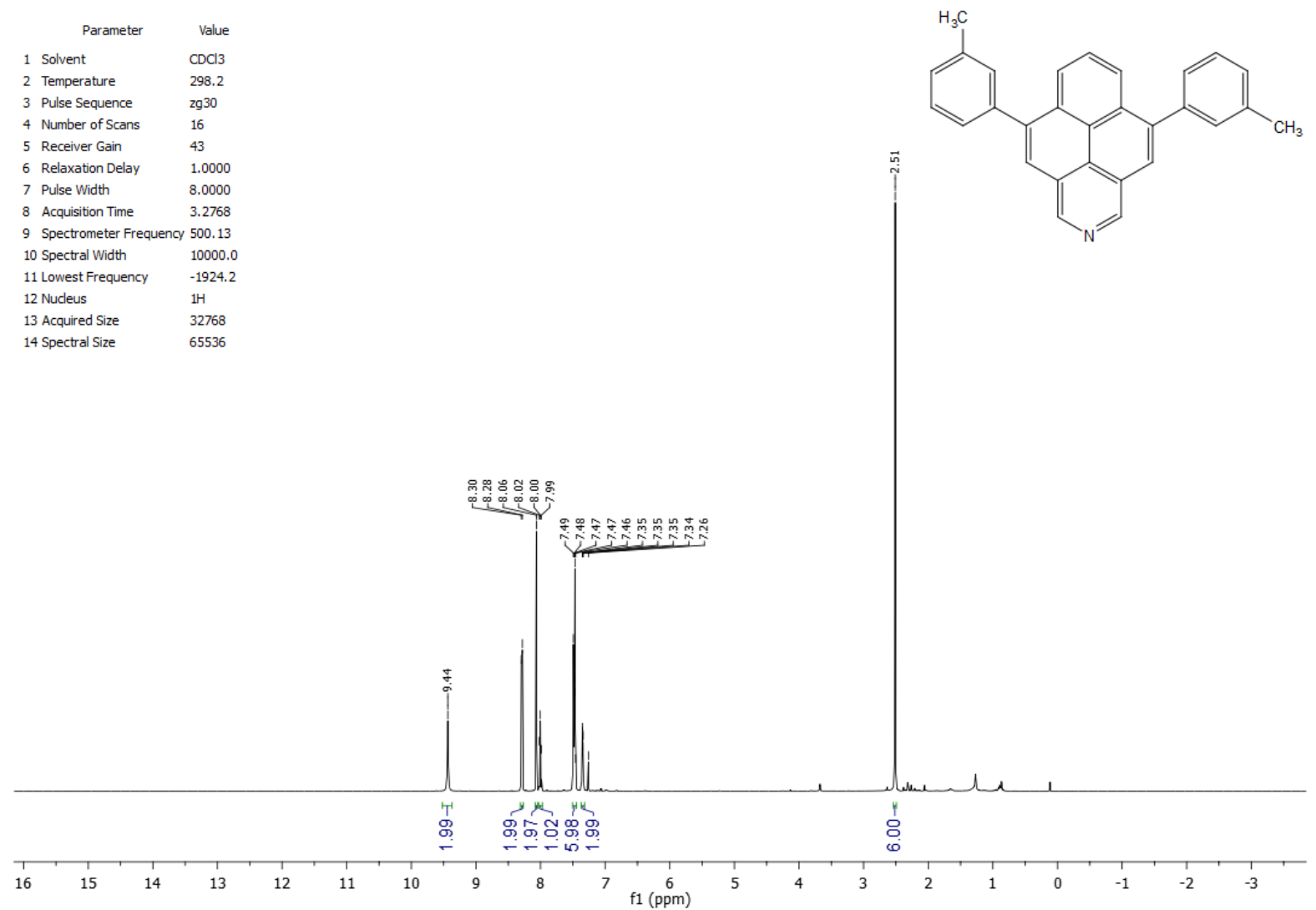

\begin{tabular}{|c|c|c|}
\hline & Parameter & Value \\
\hline & Solvent & $\mathrm{CDCl} 3$ \\
\hline & Temperature & 298.1 \\
\hline & Pulse Sequence & zgpg30 \\
\hline & Number of Scans & 1024 \\
\hline & Receiver Gain & 101 \\
\hline & Relaxation Delay & 2.0000 \\
\hline & Pulse Width & 10.0000 \\
\hline & Acquisition Time & 1.0879 \\
\hline & Spectrometer Frequency & 125.76 \\
\hline & Spectral Width & 30120.5 \\
\hline & Lowest Frequency & -2494.0 \\
\hline & Nucleus & $13 \mathrm{C}$ \\
\hline & Acquired Size & 32768 \\
\hline & Spectral Size & 32768 \\
\hline
\end{tabular}

$\begin{array}{lllllllllll}210 & 200 & 190 & 180 & 170 & 160 & 150 & 140 & 130 & 120 & 110 \underset{\mathrm{f} 1(\mathrm{ppm})}{100} 90\end{array}$

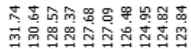

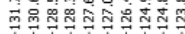

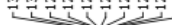

2768 
7-methyl-5,9-di-m-tolylnaphtho[2,1,8-def]isoquinoline (5f)

\begin{tabular}{lll} 
& \multicolumn{1}{c}{ Parameter } & \multicolumn{1}{c}{ Value } \\
1 Solvent & CDCl3 \\
2 2 & Temperature & 298.2 \\
3 Pulse Sequence & zg30 \\
4 4 & Number of Scans & 16 \\
5 & Receiver Gain & 144 \\
6 Relaxation Delay & 1.0000 \\
7 Pulse Width & 9.0000 \\
8 Acquisition Time & 5.2954 \\
9 Spectrometer Frequency & 300.13 \\
10 & Spectral Width & 6188.1 \\
11 Lowest Frequency & -1246.7 \\
12 Nucleus & $1 \mathrm{H}$ \\
13 Acquired Size & 32768 \\
14 Spectral Size & 32768
\end{tabular}
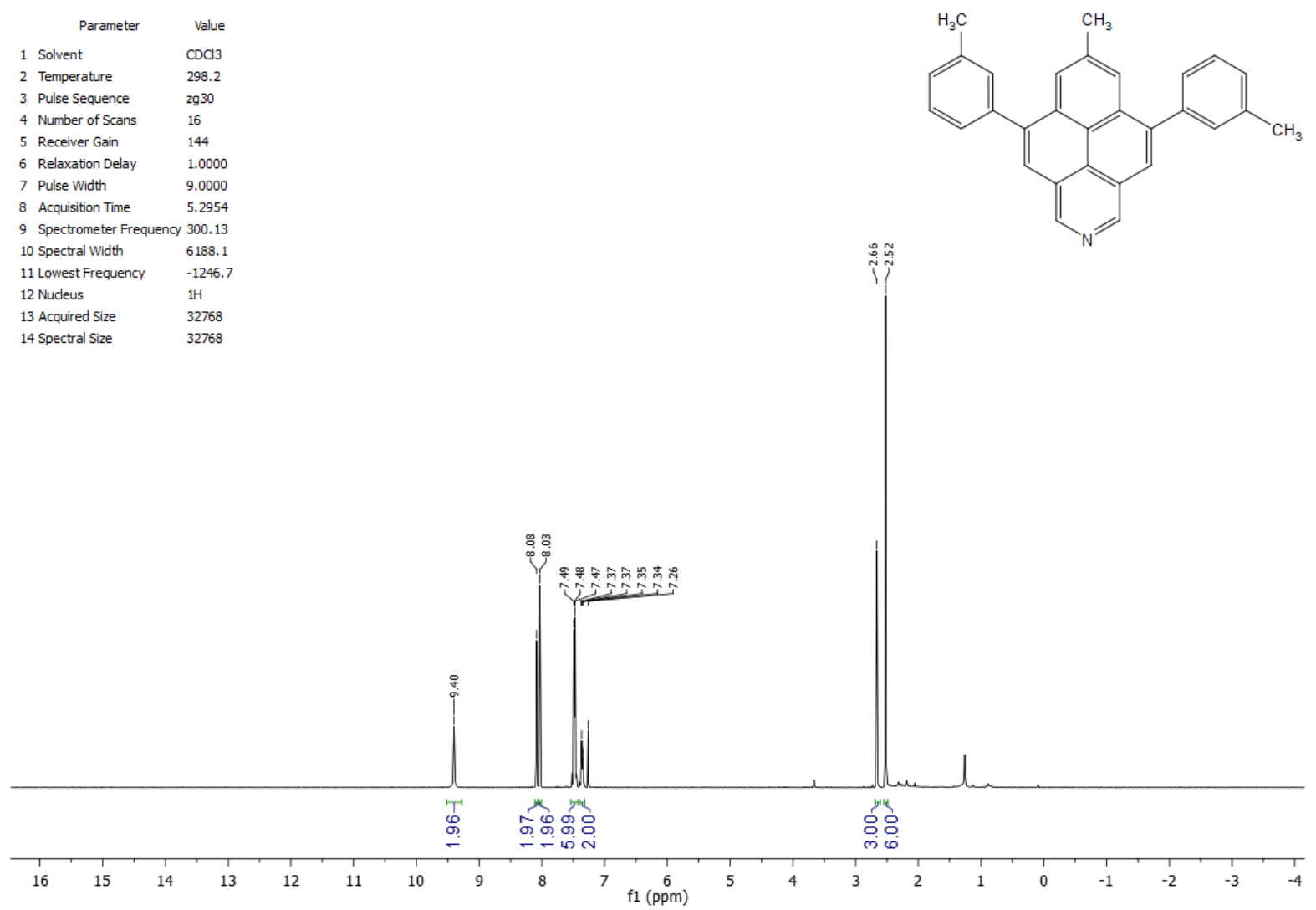

\begin{tabular}{lll}
\multicolumn{1}{c}{ Parameter } & \multicolumn{1}{c}{ Value } \\
1 Solvent & CDCl3 \\
2 Temperature & 298.2 \\
3 Pulse Sequence & $29 p 930$ \\
4 Number of Scans & 2048 \\
5 Receiver Gain & 2050 \\
6 Relaxation Delay & 2.0000 \\
7 Pulse Width & 10.0000 \\
8 Acquisition Time & 1.8176 \\
9 Spectrometer Frequency & 75.47 \\
10 Spectral Width & 18028.8 \\
11 Lowest Frequency & -1470.2 \\
12 Nucleus & $13 \mathrm{C}$ \\
13 Acquired Size & 32768 \\
14 Spectral Size & 32768
\end{tabular}

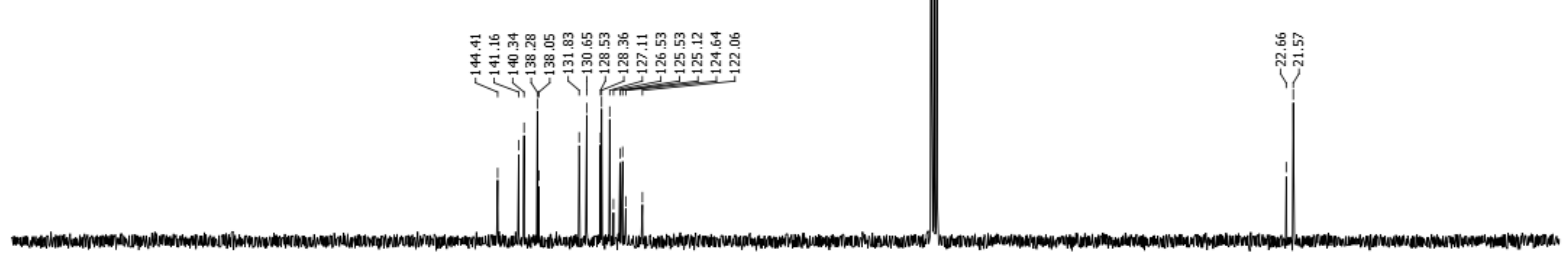

$\begin{array}{llllllllllllllllllllllllllll}210 & 200 & 190 & 180 & 170 & 160 & 150 & 140 & 130 & 120 & 110 & 100 & 90 & 80 & 70 & 60 & 50 & 40 & 30 & 20 & 10 & 0 & -10\end{array}$ 
5,9-di-p-tolylnaphtho[2,1,8-def]isoquinoline $(5 \mathrm{~g})$

\begin{tabular}{|c|c|c|}
\hline & Parameter & Valu \\
\hline & Solvent & $\mathrm{CDCl}$ \\
\hline & Temperature & 298.2 \\
\hline & Pulse Sequence & 2930 \\
\hline & Number of Scans & 16 \\
\hline & Receiver Gain & 81 \\
\hline & Relaxation Delay & 1.00 \\
\hline & Pulse Width & 9.00 \\
\hline & Acquisition Time & 5.29 \\
\hline & Spectrometer Frequency & 300. \\
\hline & Spectral Width & 618 \\
\hline & Lowest Frequency & -124 \\
\hline & Nucleus & $1 \mathrm{H}$ \\
\hline & Acquired Size & 327 \\
\hline & Spectral Size & 327 \\
\hline
\end{tabular}
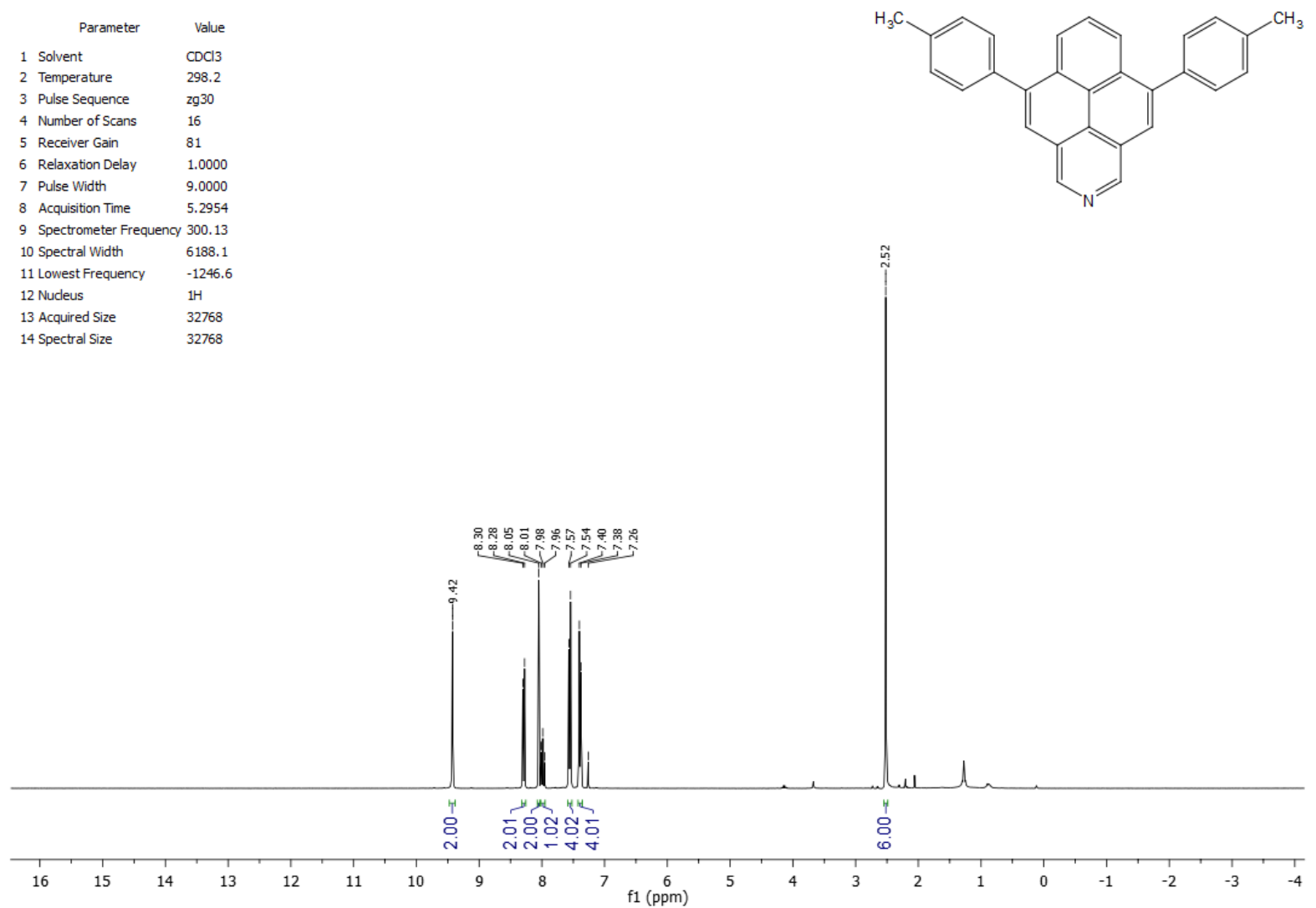

\begin{tabular}{lll}
\multicolumn{1}{c}{ Parameter } & \multicolumn{1}{c}{ Value } \\
1 Solvent & CDCl3 \\
2 Temperature & 298.2 \\
3 Pulse Sequence & 29pg30 \\
4 Number of Scans & 2048 \\
5 Receiver Gain & 2050 \\
6 Relaxation Delay & 2.0000 \\
7 Pulse Width & 10.0000 \\
8 Acquisition Time & 1.8176 \\
9 Spectrometer Frequency 75.47 \\
10 Spectral Width & 18028.8 \\
11 Lowest Frequency & -1472.4 \\
12 Nucleus & $13 C$ \\
13 Acquired Size & 32768 \\
14 Spectral Size & 32768
\end{tabular}

14 Spectral Size $\quad 32768$

$\begin{array}{lllllllllll}210 & 200 & 190 & 180 & 170 & 160 & 150 & 140 & 130 & 120 & 110 \underset{\mathrm{f} 1(\mathrm{ppm})}{100} 90\end{array}$
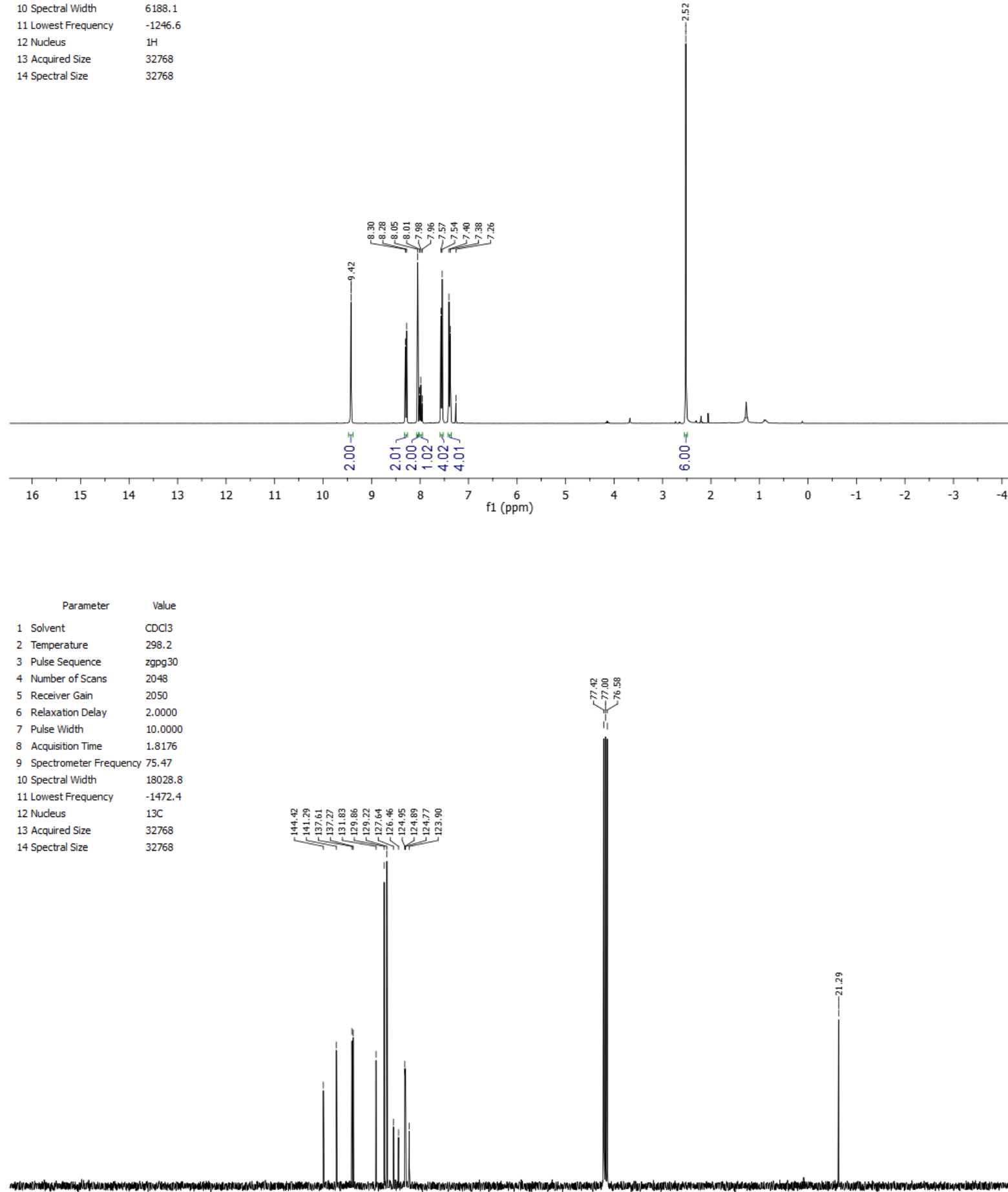
7-methyl-5,9-di-p-tolylnaphtho[2,1,8-def]isoquinoline (5h)
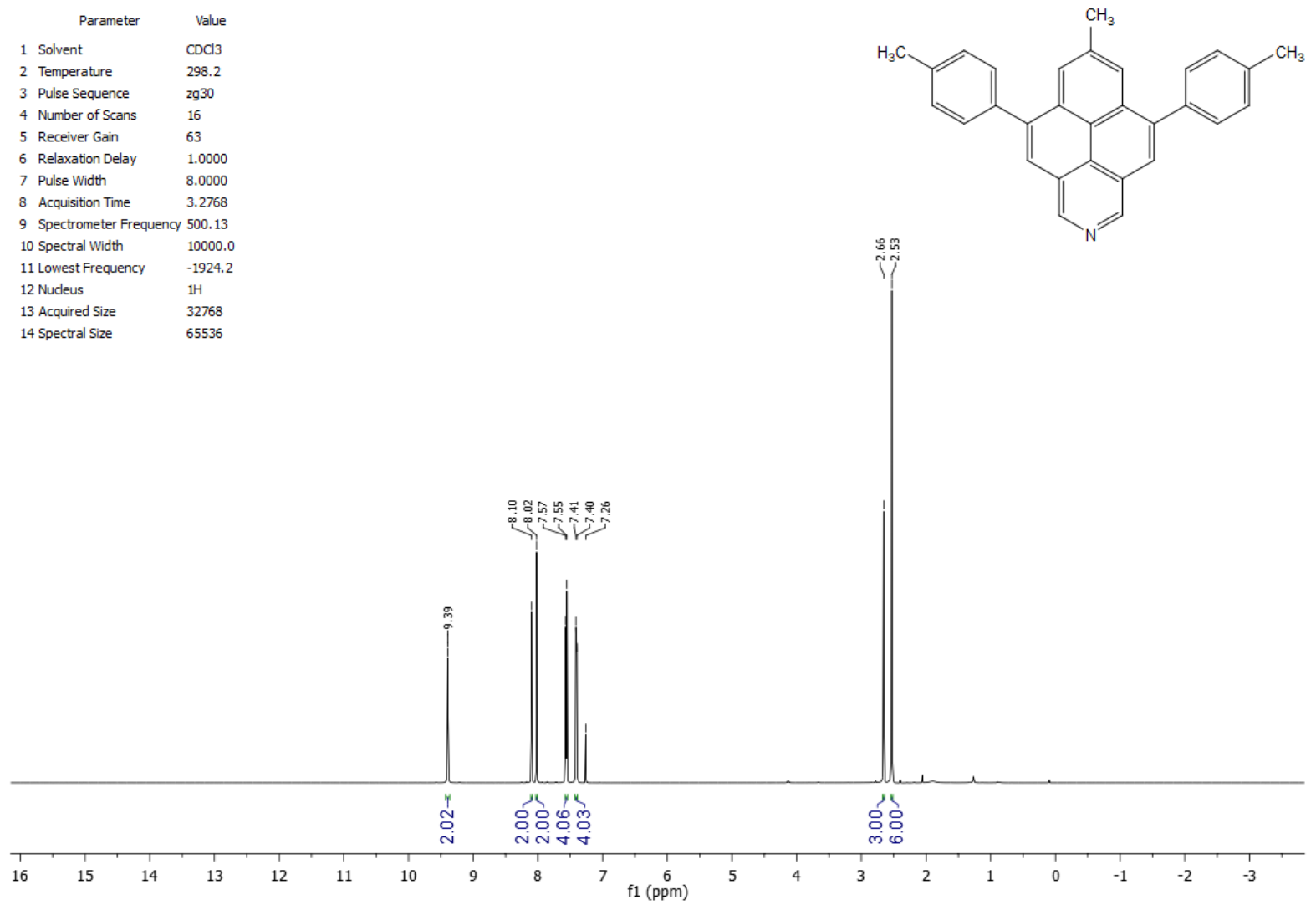

\begin{tabular}{lll}
\multicolumn{1}{c}{ Parameter } & \multicolumn{1}{c}{ Value } \\
1 Solvent & CDCl3 \\
2 2emperature & 298.2 \\
3 3 & Pulse Sequence & $29 \mathrm{gg} 30$ \\
4 Number of Scans & 1024 \\
5 Receiver Gain & 101 \\
6 Relaxation Delay & 2.0000 \\
7 Pulse Width & 10.0000 \\
8 Acquisition Time & 1.0879 \\
9 Spectrometer Frequency & 125.76 \\
10 Spectral Width & 30120.5 \\
11 Lowest Frequency & -2490.8 \\
12 Nucleus & $13 \mathrm{C}$ \\
13 Acquired Size & 32768 \\
14 Spectral Size & 32768
\end{tabular}

14 Spectral Size 32768

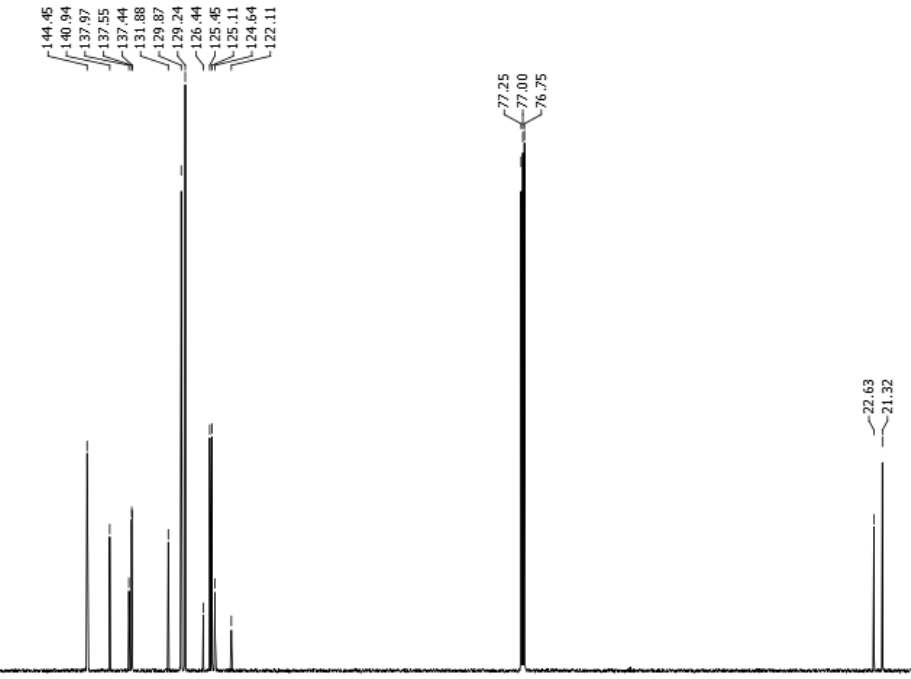

$\begin{array}{lllllllllllllllllllllll}210 & 200 & 190 & 180 & 170 & 160 & 150 & 140 & 130 & 120 & 110 & \begin{array}{c}100 \\ \mathrm{f} 1(\mathrm{ppm})\end{array} & 90 & 80 & 70 & 60 & 50 & 40 & 30 & 20 & 10 & 0 & -10\end{array}$ 
5,9-di-p-tolyl-7-(trifluoromethyl)naphtho[2,1,8-def]isoquinoline (5i)

\begin{tabular}{|c|c|c|}
\hline & Parameter & Valu \\
\hline & Solvent & $\mathrm{CDCl}$ \\
\hline & Temperature & 298. \\
\hline & Pulse Sequence & 2930 \\
\hline & Number of Scans & 16 \\
\hline & Receiver Gain & 362 \\
\hline & Relaxation Delay & 1.00 \\
\hline & Pulse Width & 10.0 \\
\hline & Acquisition Time & 6.34 \\
\hline & Spectrometer Frequency & 250. \\
\hline & Spectral Width & 5165 \\
\hline & Lowest Frequency & \\
\hline & Nucleus & \\
\hline & Iqquired Size & \\
\hline & pectral Size & \\
\hline
\end{tabular}
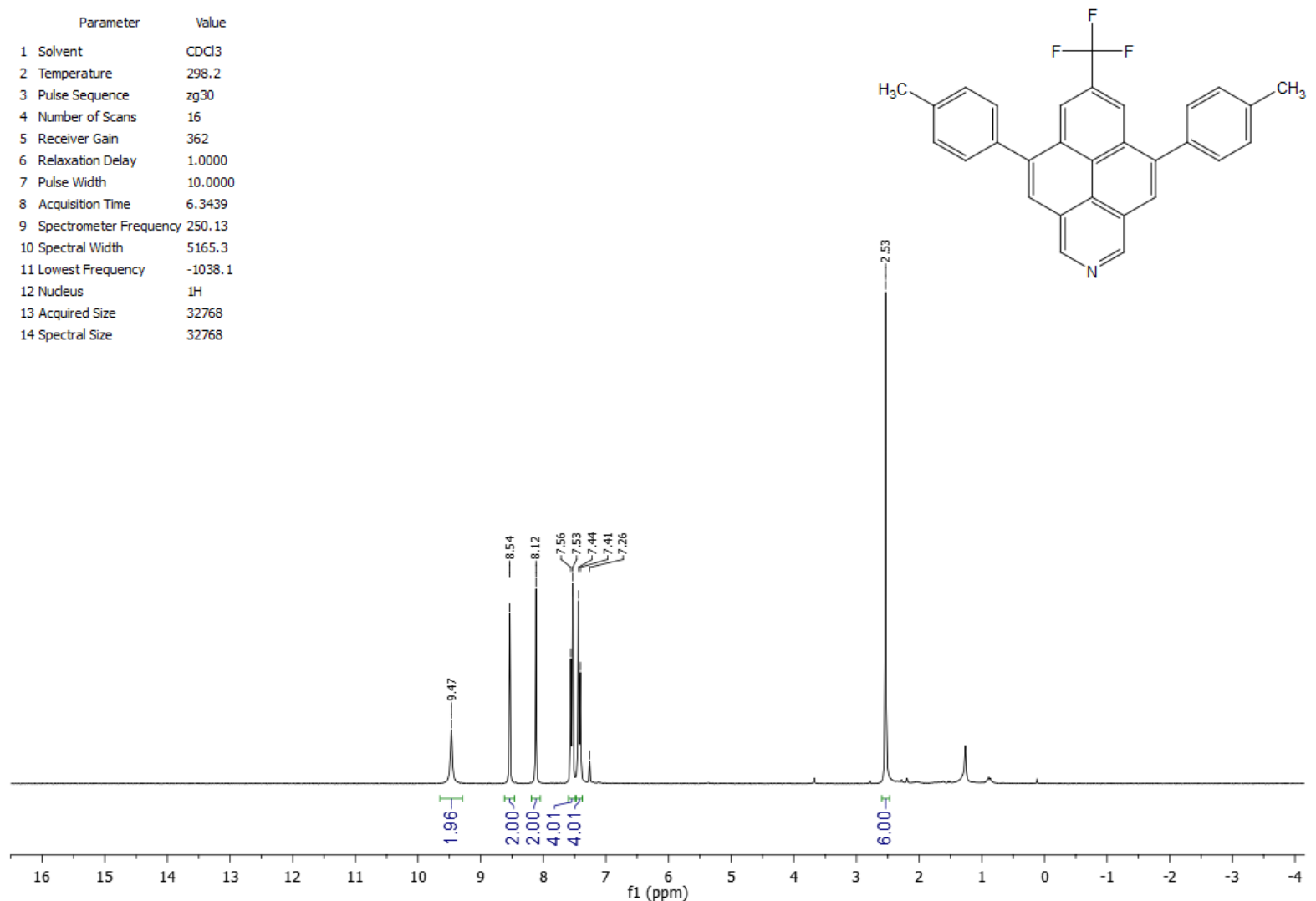

\begin{tabular}{|c|c|c|}
\hline & Parameter & Value \\
\hline & Solvent & $\mathrm{CDCl} 3$ \\
\hline & Temperature & 298.2 \\
\hline & Pulse Sequence & zgfhigan \\
\hline & Number of Scans & 64 \\
\hline & Receiver Gain & 2050 \\
\hline & Relaxation Delay & 1.0000 \\
\hline & Pulse Width & 10.0000 \\
\hline & Acquisition Time & 0.9787 \\
\hline & Spectrometer Frequency & 282.40 \\
\hline & Spectral Width & 66964.3 \\
\hline & Lowest Frequency & -61722.4 \\
\hline & Nucleus & $19 F$ \\
\hline & Acquired Size & 65536 \\
\hline & Spectral Size & 65536 \\
\hline
\end{tabular}




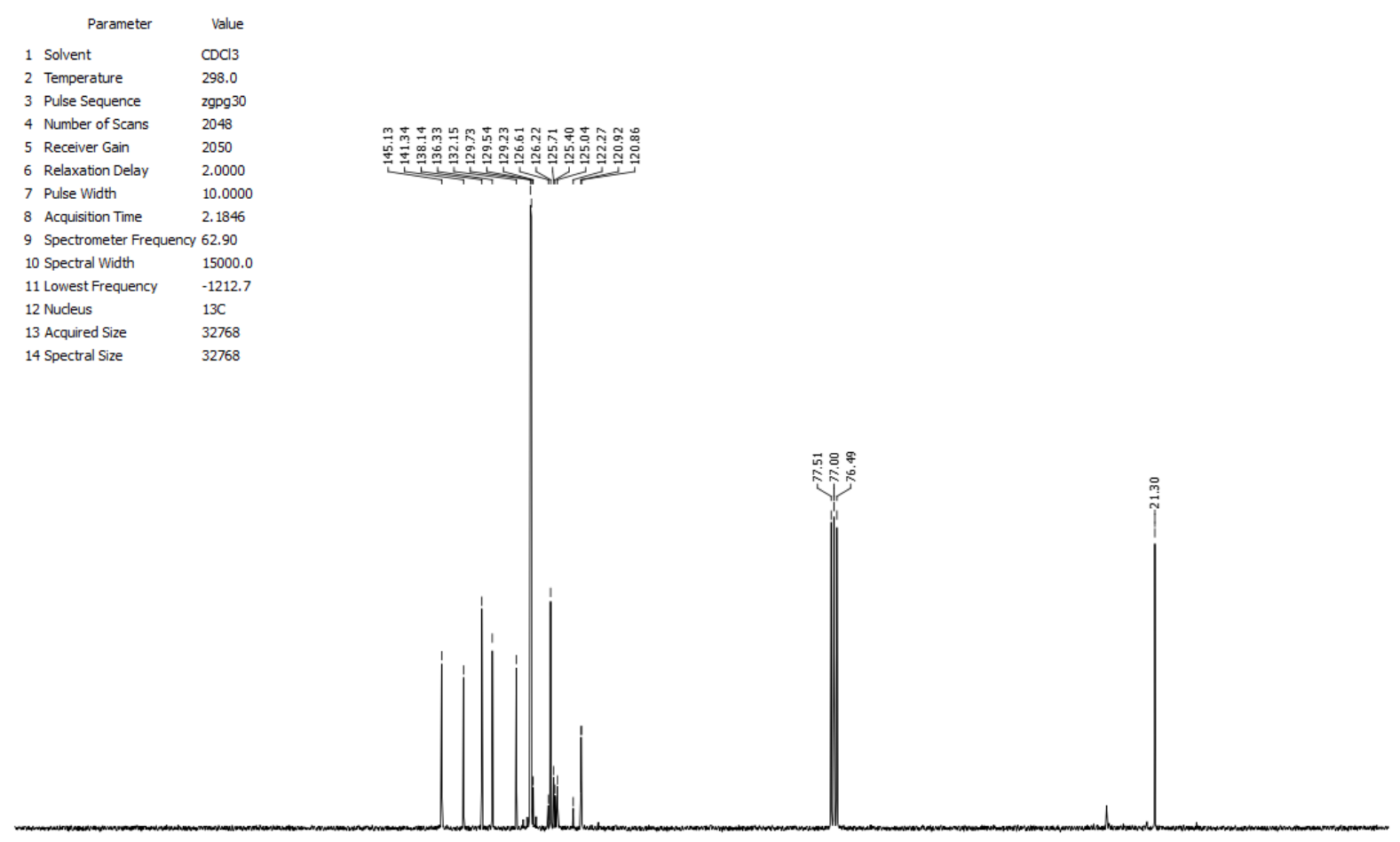

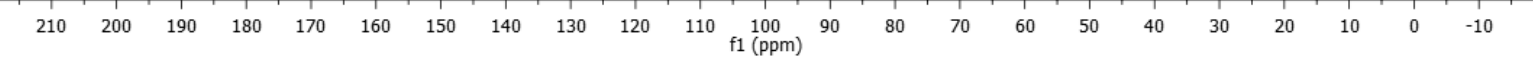




\section{5,9-bis(4-fluorophenyl)naphtho[2,1,8-def]isoquinoline (5j)}

\begin{tabular}{|c|c|c|}
\hline & Parameter & Valu \\
\hline & Solvent & $\mathrm{CDCl}$ \\
\hline & Temperature & 298.2 \\
\hline & Pulse Sequence & 2930 \\
\hline & Number of Scans & 16 \\
\hline & Receiver Gain & 144 \\
\hline & Relaxation Delay & 1.00 \\
\hline & Pulse Width & 10.0 \\
\hline & Acquisition Time & 5.29 \\
\hline & Spectrometer Frequency & 300. \\
\hline & Spectral Width & 6188 \\
\hline & Lowest Frequency & \\
\hline & Nucleus & $1 \mathrm{H}$ \\
\hline & Acquired Size & 327 \\
\hline & Spectral Size & \\
\hline
\end{tabular}
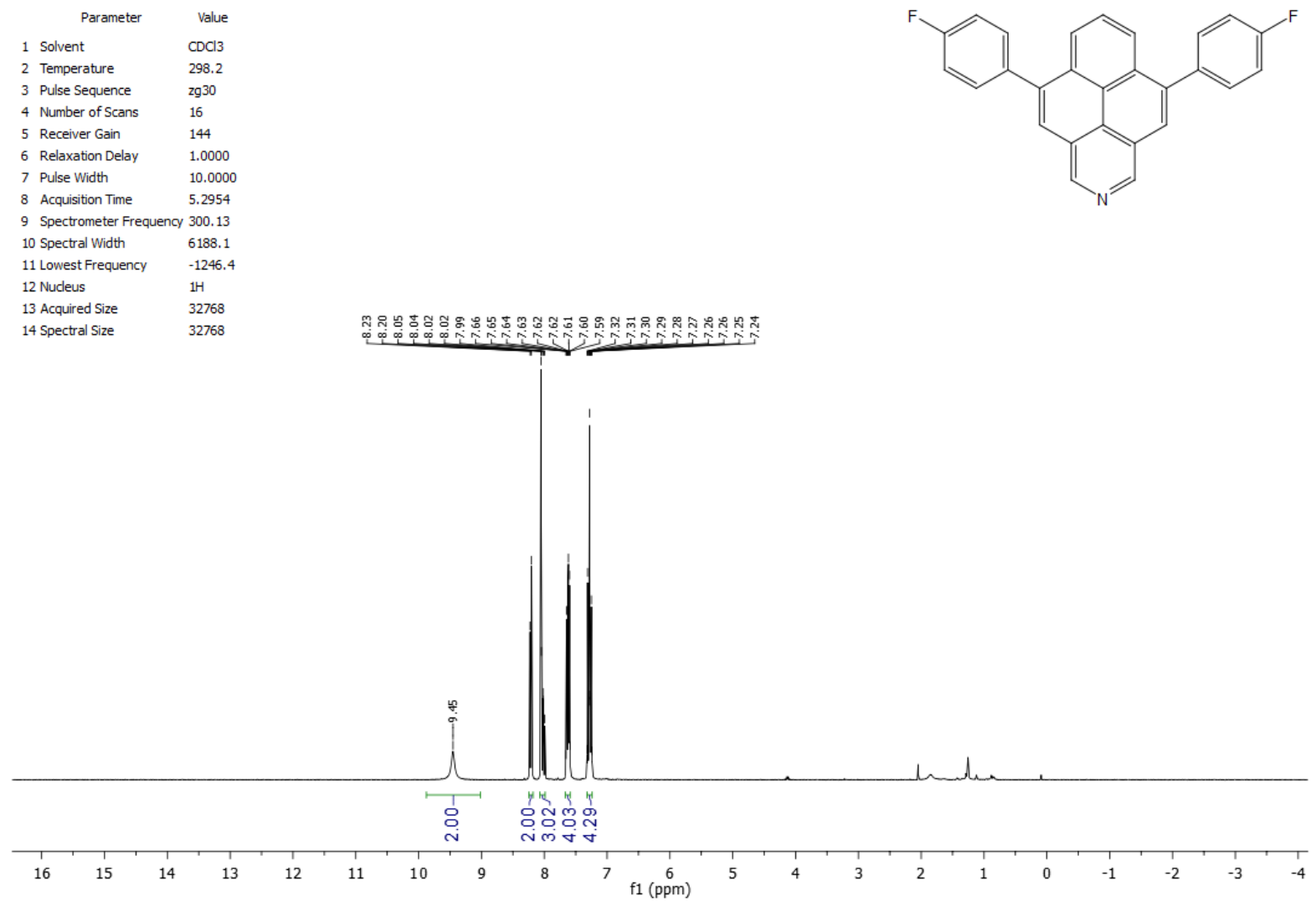

\begin{tabular}{lll}
\multicolumn{1}{c}{ Parameter } & \multicolumn{1}{c}{ Value } \\
1 Solvent & CDCl3 \\
2 2emperature & 298.2 \\
3 Pulse Sequence & 2gfhiggn \\
4 Number of Scans & 64 \\
5 Receiver Gain & 2050 \\
6 Relaxation Delay & 1.0000 \\
7 Pulse Width & 10.0000 \\
8 Acquisition Time & 0.9787 \\
9 Spectrometer Frequency & 282.40 \\
10 Spectral Width & 66964.3 \\
11 Lowest Frequency & -61722.4 \\
12 Nucleus & $19 F$ \\
13 Acquired Size & 65536 \\
14 Spectral Size & 65536
\end{tabular}




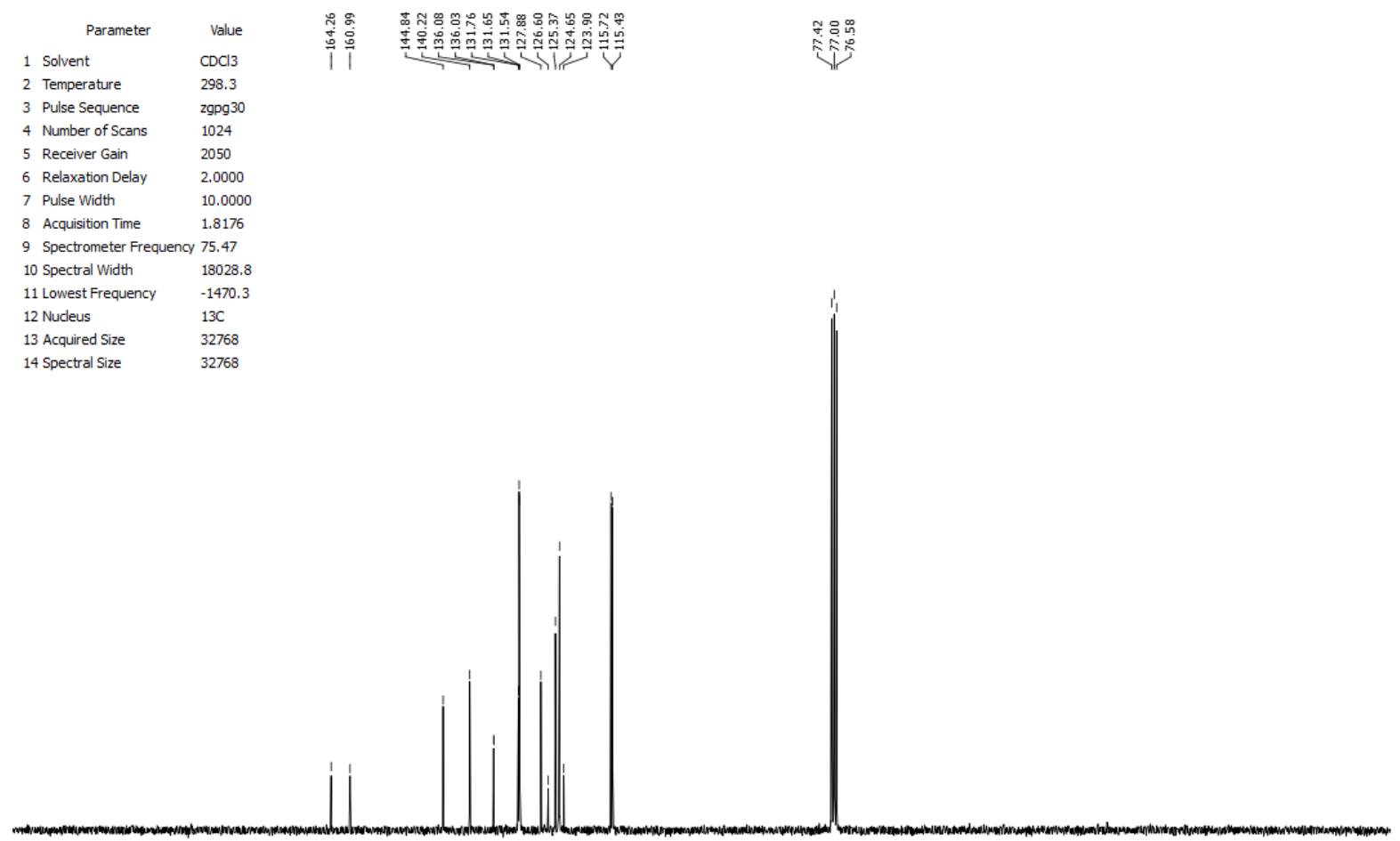

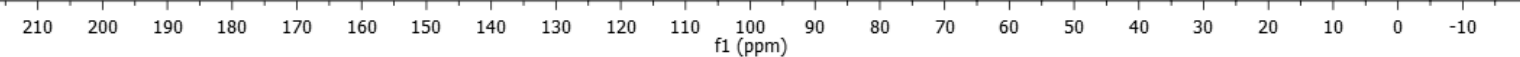


5,9-bis(4-fluorophenyl)-7-methylnaphtho[2,1,8-def]isoquinoline (5k)
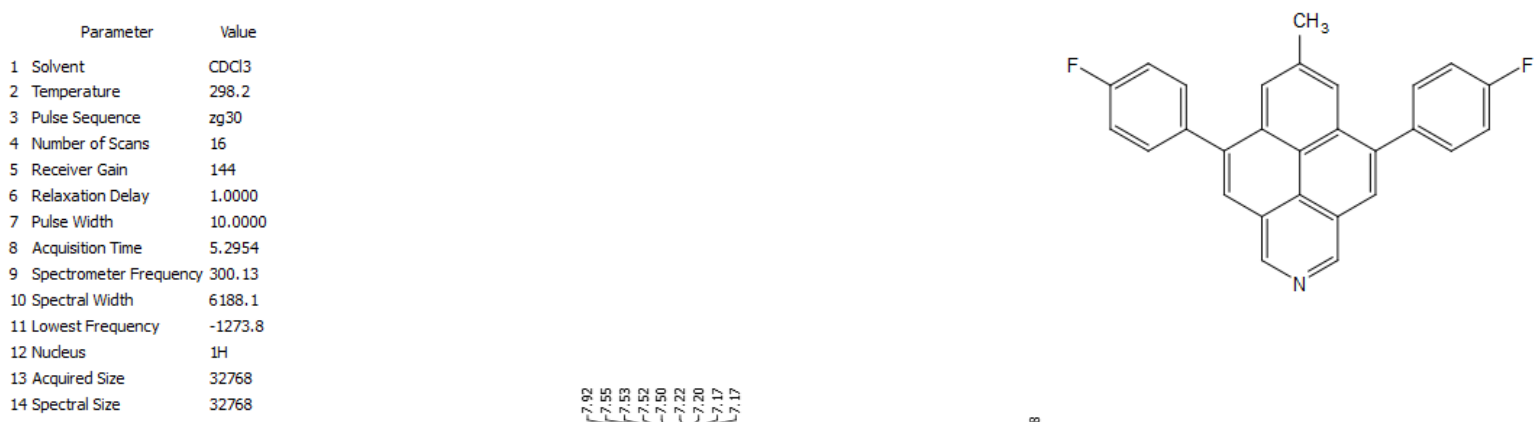

14 Spectral Size $\quad 32760$

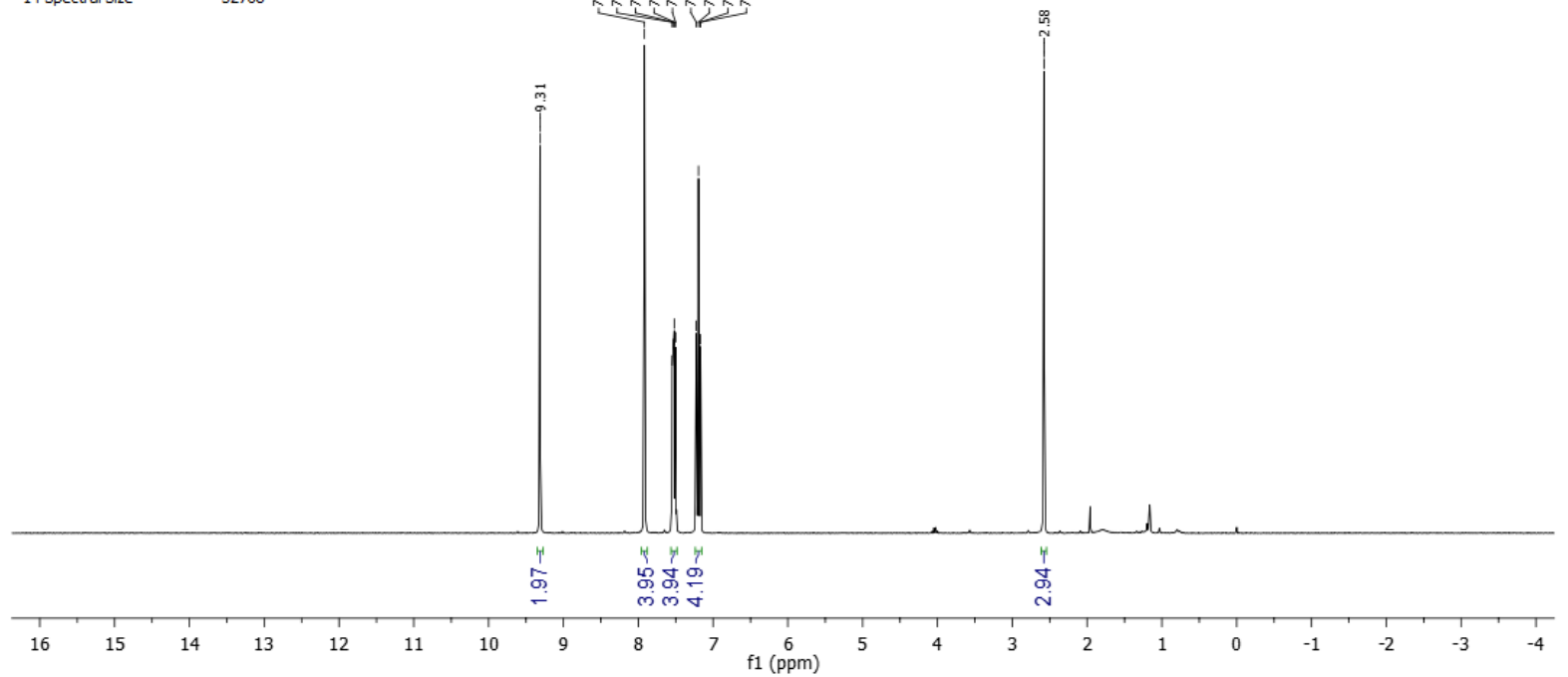

\begin{tabular}{|c|c|c|}
\hline & Parameter & Value \\
\hline & Solvent & $\mathrm{CDCl} 3$ \\
\hline & Temperature & 298.2 \\
\hline & Pulse Sequence & zgfhiggn \\
\hline & Number of Scans & 64 \\
\hline & Receiver Gain & 2050 \\
\hline & Relaxation Delay & 1.0000 \\
\hline & Pulse Width & 10.0000 \\
\hline & Acquisition Time & 0.9787 \\
\hline & Spectrometer Frequency & 282.40 \\
\hline & Spectral Width & 66964.3 \\
\hline & Lowest Frequency & -61722.4 \\
\hline & Nucleus & $19 F$ \\
\hline & Acquired Size & 65536 \\
\hline & Spectral Size & 65536 \\
\hline
\end{tabular}




$$
\begin{array}{lll} 
& \multicolumn{1}{c}{\text { Parameter }} & \multicolumn{1}{c}{\text { Value }} \\
1 & \text { Solvent } & \text { CDCl3 } \\
2 & \text { Temperature } & 298.2 \\
3 & \text { Pulse Sequence } & \text { zgpg } 30 \\
4 & \text { Number of Scans } & 1024 \\
5 & \text { Receiver Gain } & 2050 \\
6 & \text { Relaxation Delay } & 2.0000 \\
7 & \text { Pulse Width } & 10.0000 \\
8 & \text { Acquisition Time } & 1.8176 \\
9 & \text { Spectrometer Frequency } & 75.47 \\
10 & \text { Spectral Width } & 18028.8 \\
\text { 11 } \text { Lowest Frequency } & -1470.3 \\
\text { 12 } & \text { Nucleus } & 13 \mathrm{C} \\
\text { 13 } \text { Acquired Size } & 32768 \\
\text { 14 Spectral Size } & 32768
\end{array}
$$

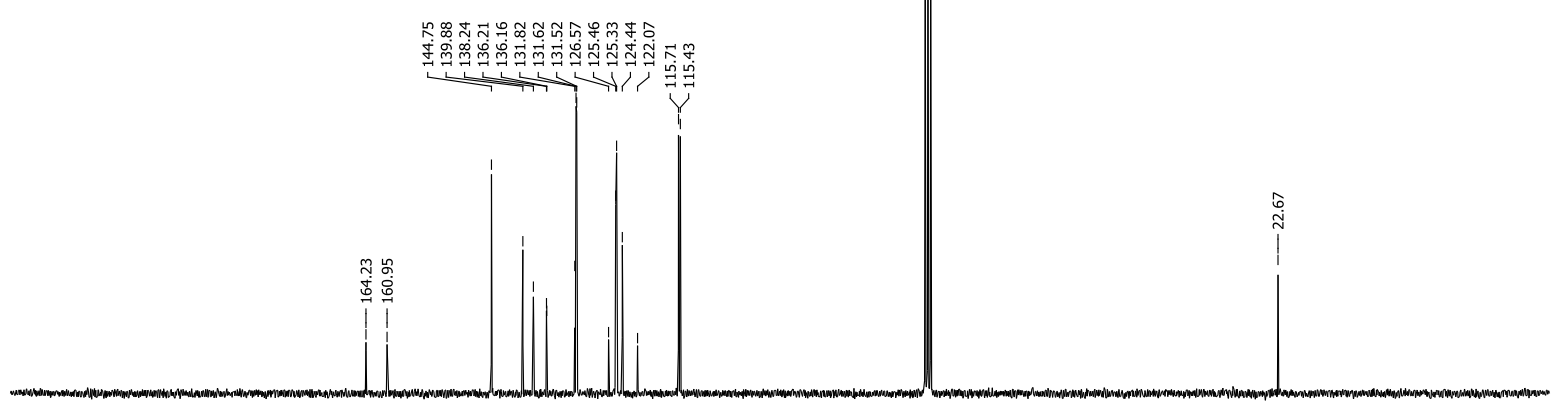

$\begin{array}{llllllllllllllllllllllll}210 & 200 & 190 & 180 & 170 & 160 & 150 & 140 & 130 & 120 & 110 & \begin{array}{l}100 \\ 100\end{array} & 90 & 80 & 70 & 60 & 50 & 40 & 30 & 20 & 10 & 0 & -10\end{array}$ 
5,9-bis(4-fluorophenyl)naphtho[2,1,8-def]isoquinolin-7-ol (5I)

\begin{tabular}{lll}
\multicolumn{1}{c}{ Parameter } & \multicolumn{1}{c}{ Value } \\
1 Solvent & CDCl3 \\
2 2 & Temperature & 298.2 \\
3 Pulse Sequence & 2930 \\
4 Number of Scans & 16 \\
5 Receiver Gain & 256 \\
6 Relaxation Delay & 2.0000 \\
7 Pulse Width & 10.0000 \\
8 Acquisition Time & 5.2954 \\
9 Spectrometer Frequency & 300.13 \\
10 & Spectral Width & 6188.1 \\
11 Lowest Frequency & -1246.2 \\
12 Nucleus & $1 \mathrm{H}$ \\
13 Acquired Size & 32768 \\
14 Spectral Size & 32768
\end{tabular}
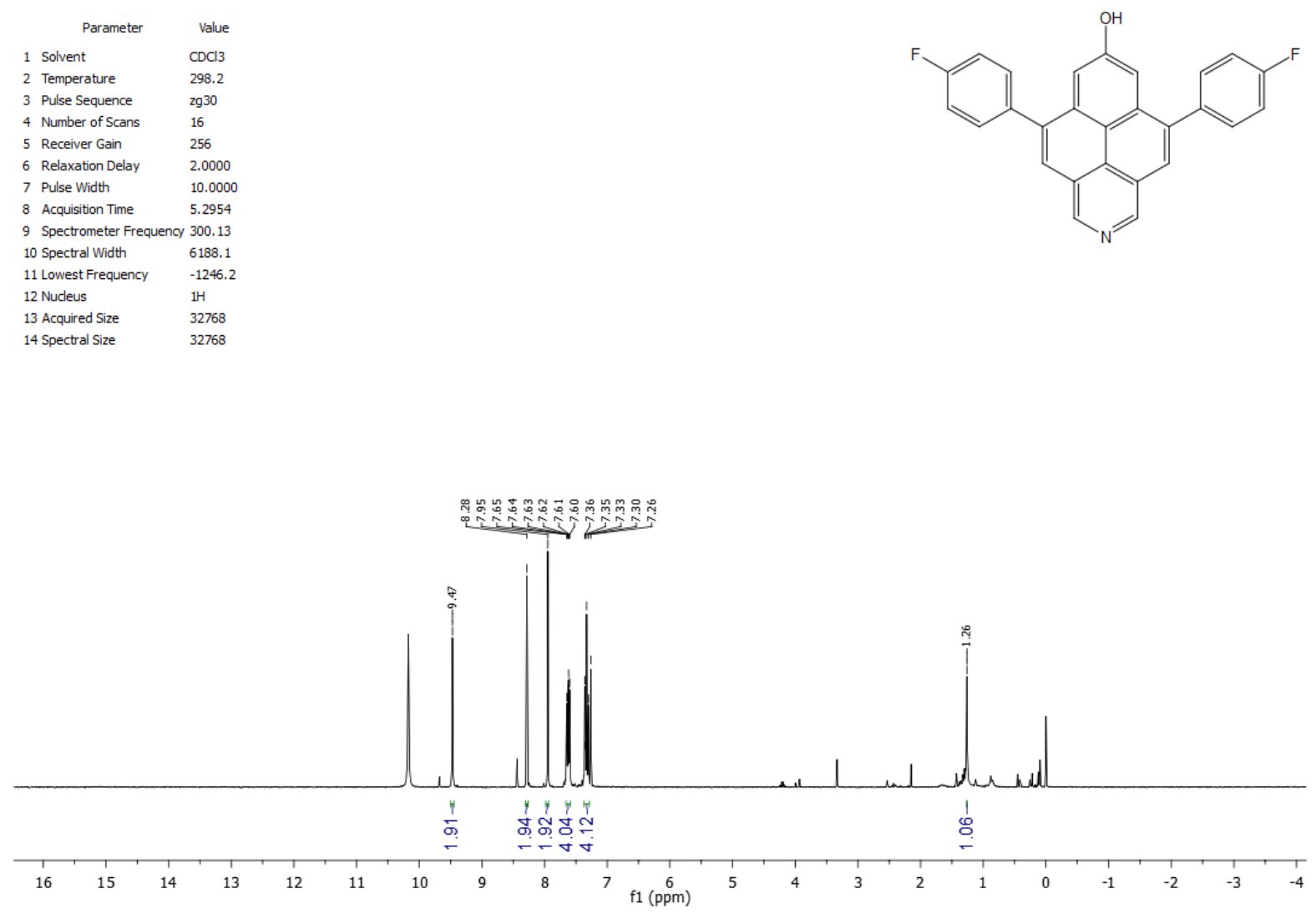

\begin{tabular}{lll} 
& \multicolumn{1}{c}{ Parameter } & \multicolumn{1}{c}{ Value } \\
1 Solvent & \multicolumn{1}{c}{ CDCl3 } \\
2 2 & Temperature & 298.2 \\
3 & Pulse Sequence & zgfhigqn \\
4 & Number of Scans & 64 \\
5 & Receiver Gain & 724 \\
6 & Relaxation Delay & 1.0000 \\
7 & Pulse Width & 10.0000 \\
8 & Acquisition Time & 0.9787 \\
9 & Spectrometer Frequency 282.40 \\
10 & Spectral Width & 66964.3 \\
11 Lowest Frequency & -61722.4 \\
12 Nucleus & $19 F$ \\
13 Acquired Size & 65536 \\
14 Spectral Size & 65536
\end{tabular}

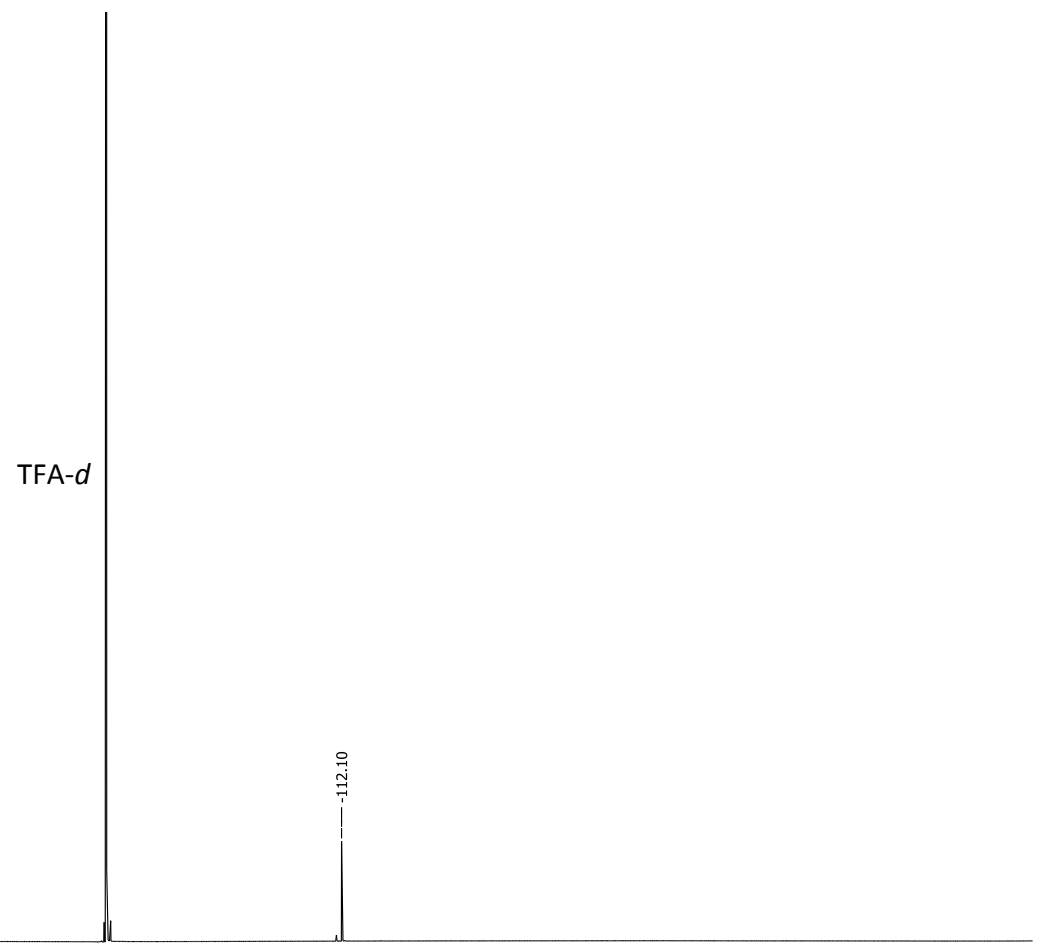

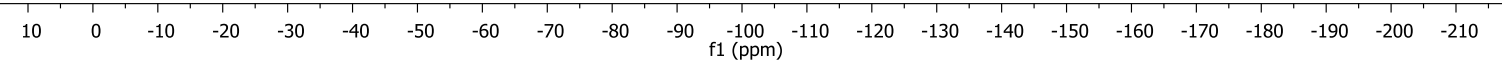




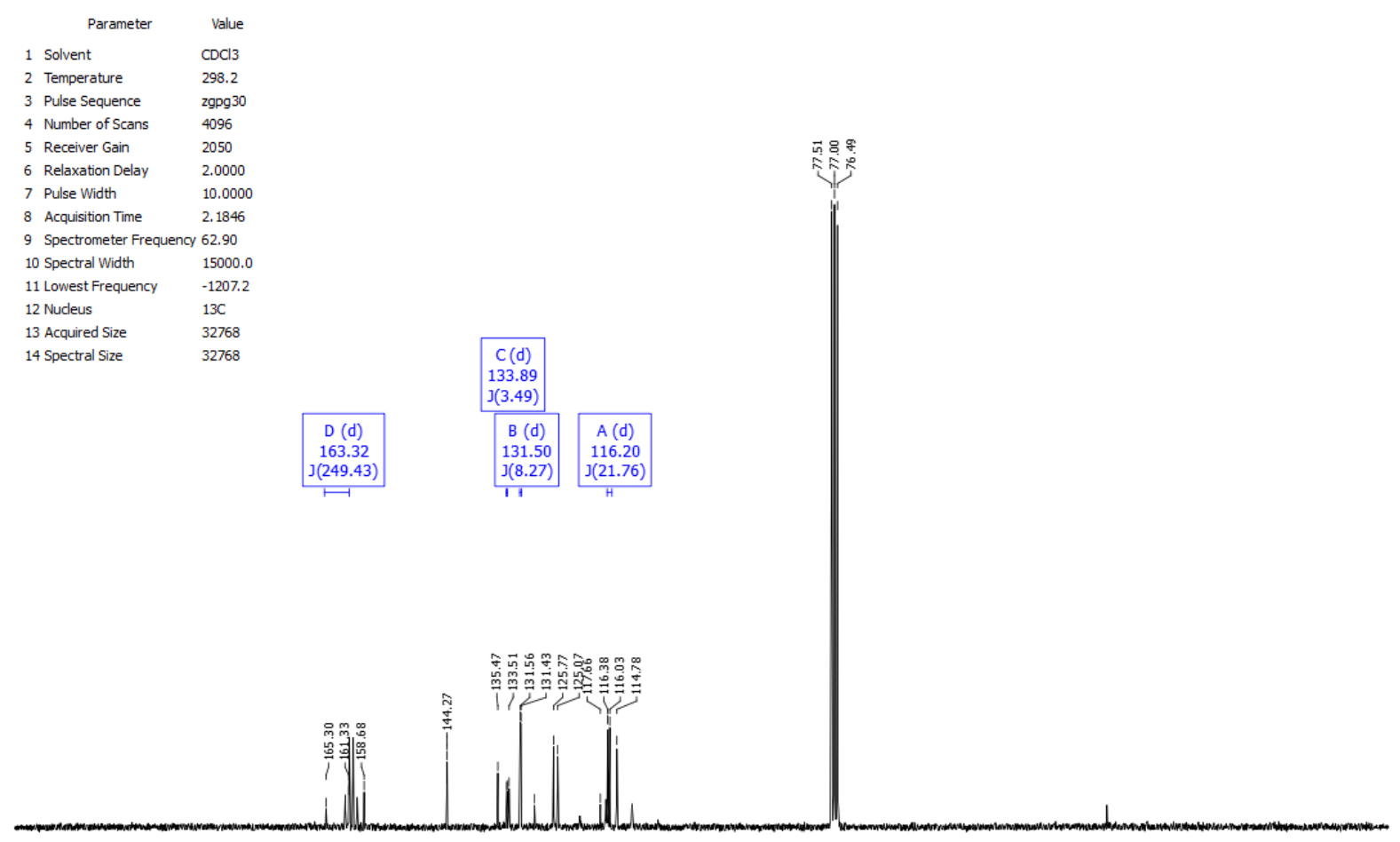

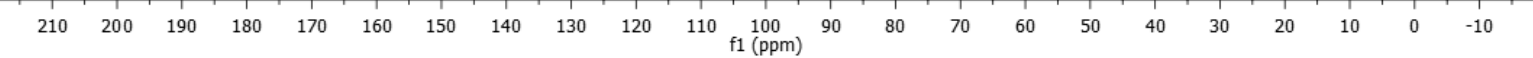


5,9-bis(4-methoxyphenyl)naphtho[2,1,8-def]isoquinoline (5m)

\begin{tabular}{lll}
\multicolumn{1}{c}{ Parameter } & \multicolumn{1}{c}{ Value } \\
1 Solvent & CDCl3 \\
2 Temperature & 298.2 \\
3 & Pulse Sequence & 2930 \\
4 Number of Scans & 16 \\
5 5 & Receiver Gain & 362 \\
6 6elaxation Delay & 1.0000 \\
7 Pulse Width & 10.0000 \\
8 & Acquisition Time & 6.3439 \\
9 Spectrometer Frequency 250.13 \\
10 Spectral Width & 5165.3 \\
11 Lowest Frequency & -1038.5 \\
12 Nucleus & $1 \mathrm{H}$ \\
13 Acquired Size & 32768 \\
14 Spectral Size & 32768
\end{tabular}
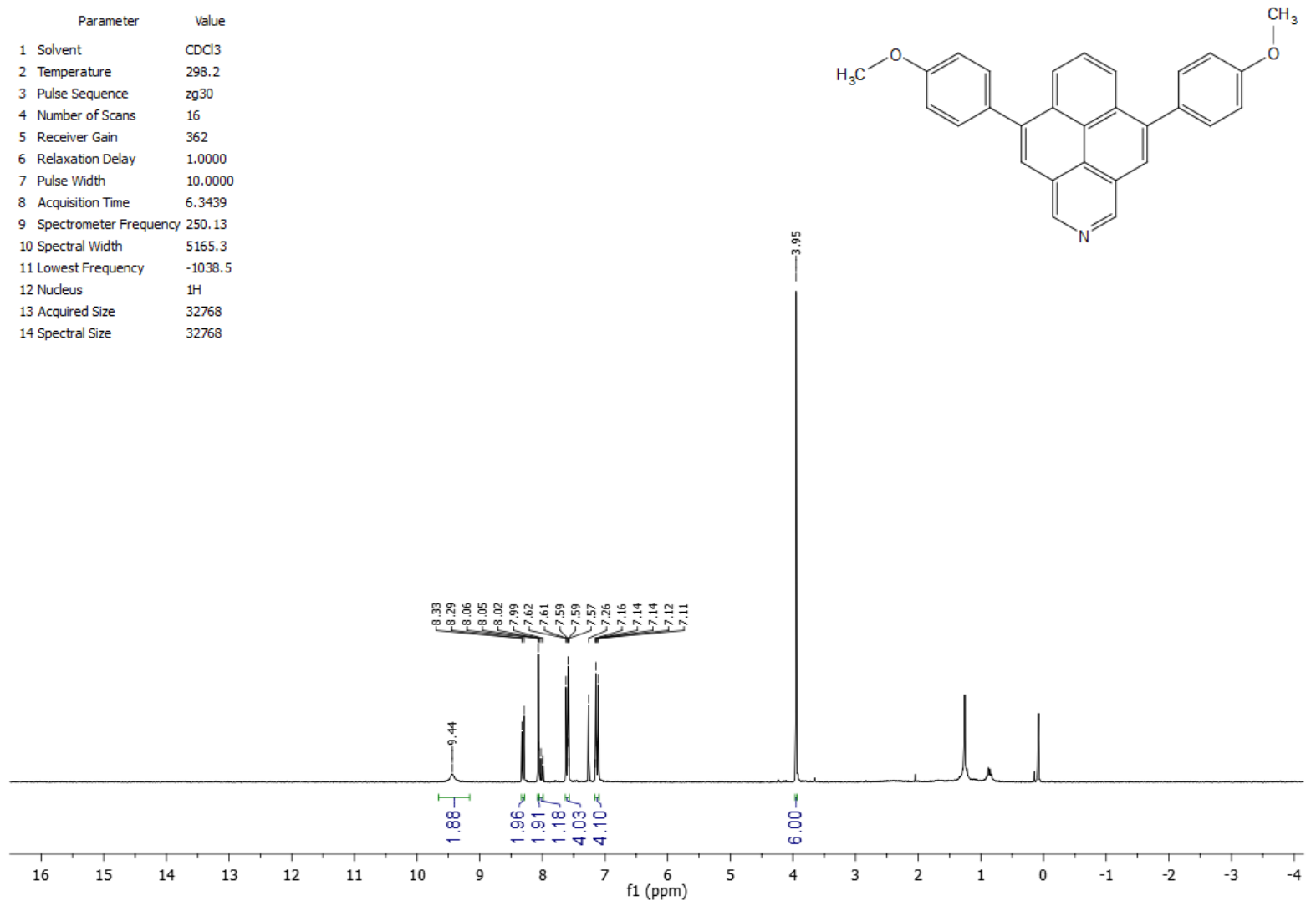

\begin{tabular}{lll}
\multicolumn{1}{c}{ Parameter } & \multicolumn{1}{c}{ Value } \\
1 Solvent & $\mathrm{CDCl} 3$ \\
2 2emperature & 297.8 \\
3 Pulse Sequence & $29 \mathrm{gg} 30$ \\
4 Number of Scans & 1024 \\
5 Receiver Gain & 2050 \\
6 Relaxation Delay & 2.0000 \\
7 Pulse Width & 10.0000 \\
8 Acquisition Time & 2.1846 \\
9 Spectrometer Frequency 62.90 \\
10 Spectral Width & 15000.0 \\
11 Lowest Frequency & -1210.1 \\
12 Nudeus & $13 \mathrm{C}$ \\
13 Acquired Size & 32768 \\
14 Spectral Size & 32768
\end{tabular}

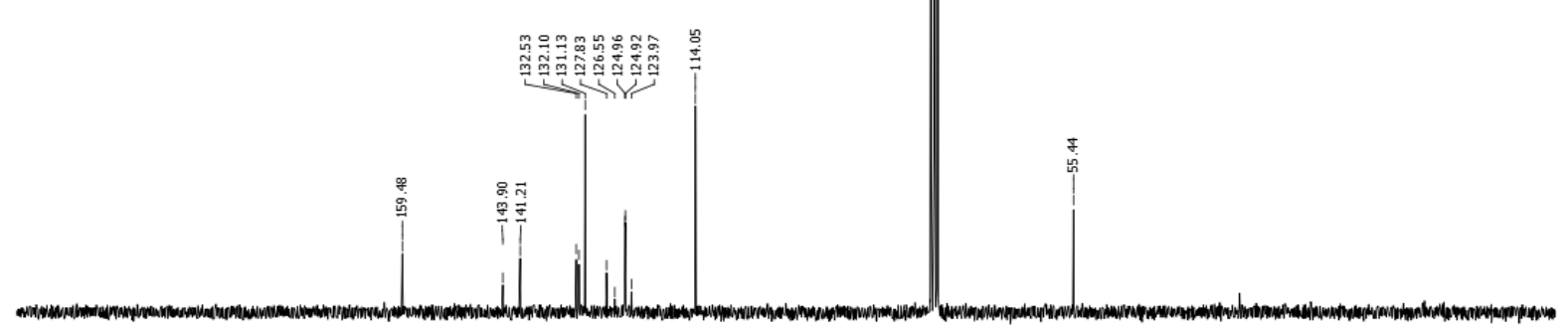

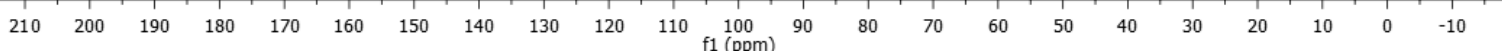


4,4'-(naphtho[2,1,8-def] isoquinoline-5,9-diyl)bis( $N, N$-dimethylaniline) (5n)

\begin{tabular}{lll} 
& \multicolumn{1}{c}{ Parameter } & \multicolumn{1}{c}{ Value } \\
1 Solvent & CDCl3 \\
2 2 & Temperature & 298.1 \\
3 & Pulse Sequence & 2930 \\
4 & Number of Scans & 16 \\
5 & Receiver Gain & 101 \\
6 & Relaxation Delay & 1.0000 \\
7 Pulse Width & 8.0000 \\
8 Acquisition Time & 3.2768 \\
9 & Spectrometer Frequency 500.13 \\
10 & Spectral Width & 10000.0 \\
11 Lowest Frequency & -1923.7 \\
12 Nucleus & $1 \mathrm{H}$ \\
13 & Acquired Size & 32768 \\
14 Spectral Size & 65536
\end{tabular}
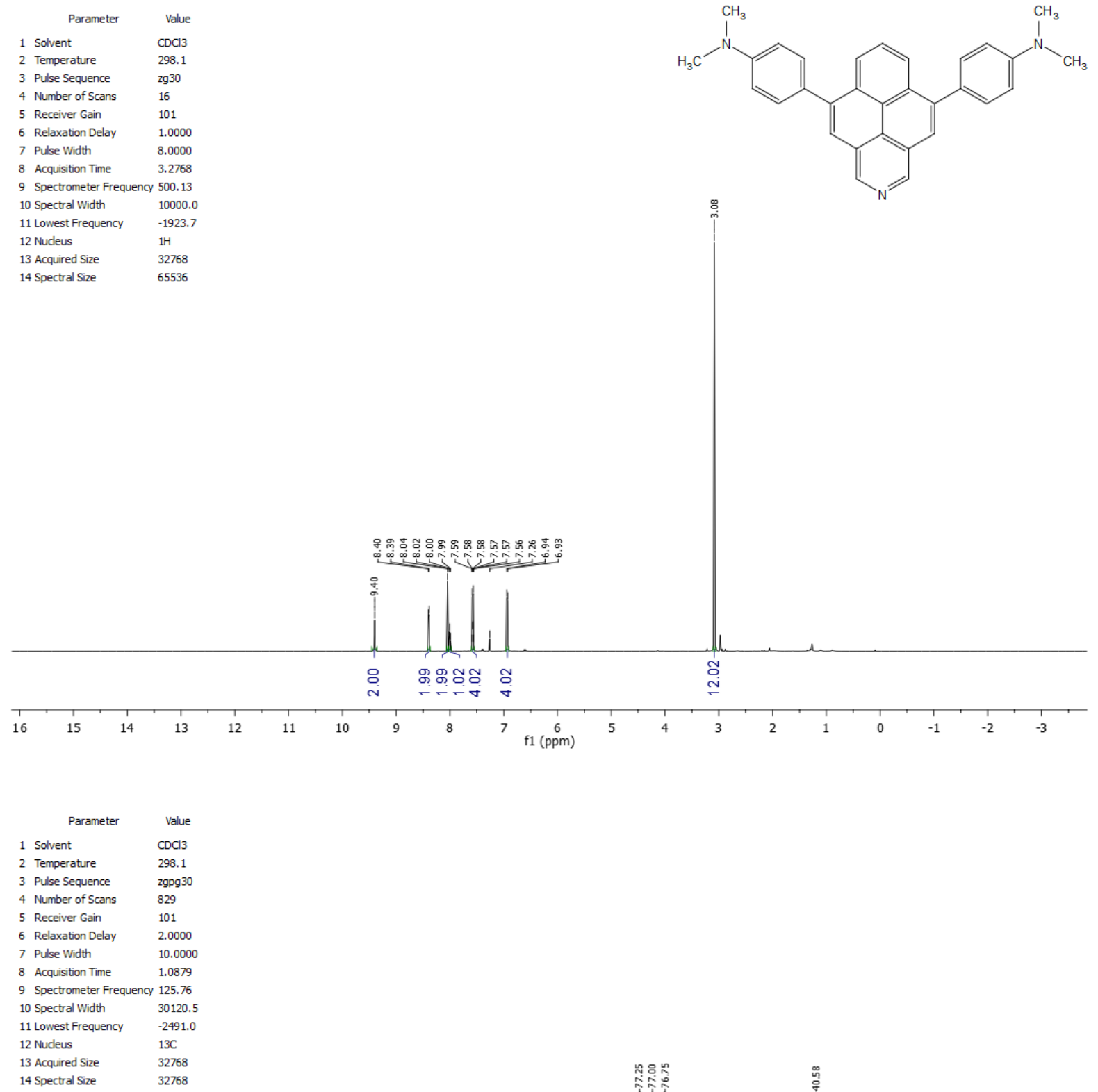

14 Spectral Size $\quad 32768$

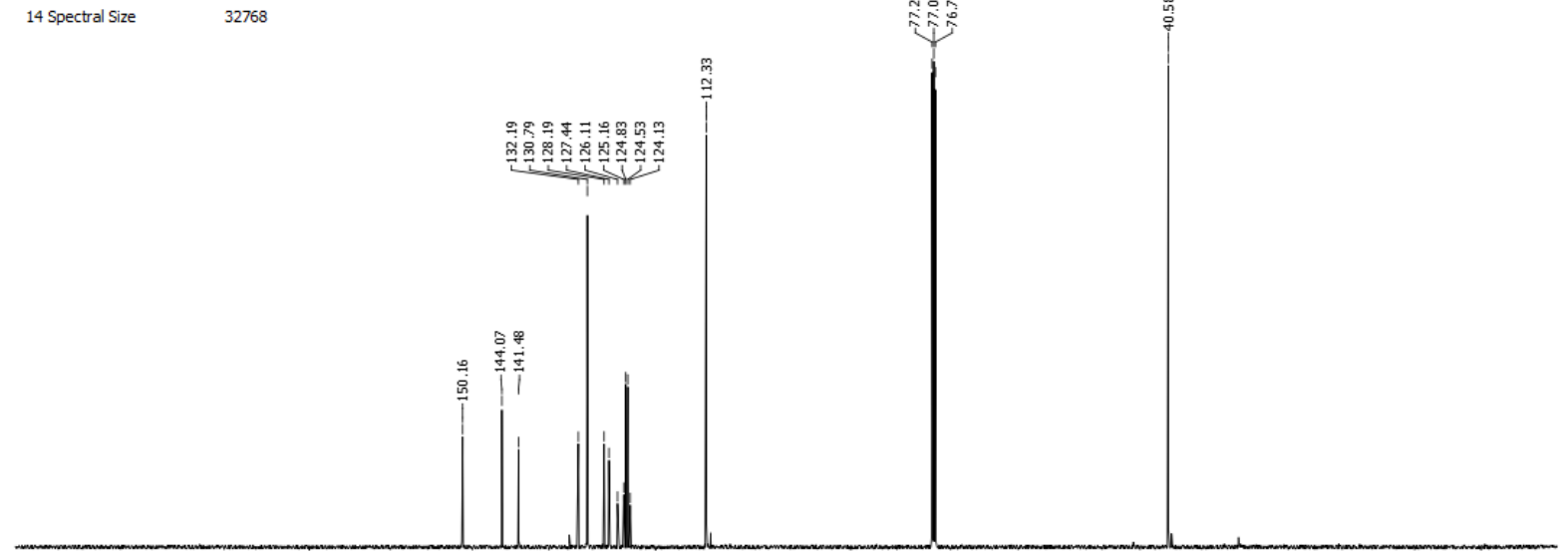

$\begin{array}{llllllllllllllllllllllll}210 & 200 & 190 & 180 & 170 & 160 & 150 & 140 & 130 & 120 & 110 & \begin{array}{c}100 \\ \mathrm{f} 1(\mathrm{ppm})\end{array} & 90 & 80 & 70 & 60 & 50 & 40 & 30 & 20 & 10 & 0 & -10\end{array}$ 
5,9-di(thiophen-3-yl)naphtho[2,1,8-def]isoquinoline (5o)

\begin{tabular}{|c|c|c|}
\hline & Parameter & Value \\
\hline & Solvent & $\mathrm{CDCl} 3$ \\
\hline & Temperature & 298.2 \\
\hline & Pulse Sequence & zg30 \\
\hline & Number of Scans & 16 \\
\hline & Receiver Gain & 228 \\
\hline & Relaxation Delay & 1.0000 \\
\hline & Pulse Width & 10.0000 \\
\hline & Acquisition Time & 5.2954 \\
\hline & Spectrometer Frequency & 300.13 \\
\hline & Spectral Width & 6188.1 \\
\hline & Lowest Frequency & -1246.5 \\
\hline & Nucleus & $1 \mathrm{H}$ \\
\hline & Acquired Size & 32768 \\
\hline & Spectral Size & 32768 \\
\hline
\end{tabular}
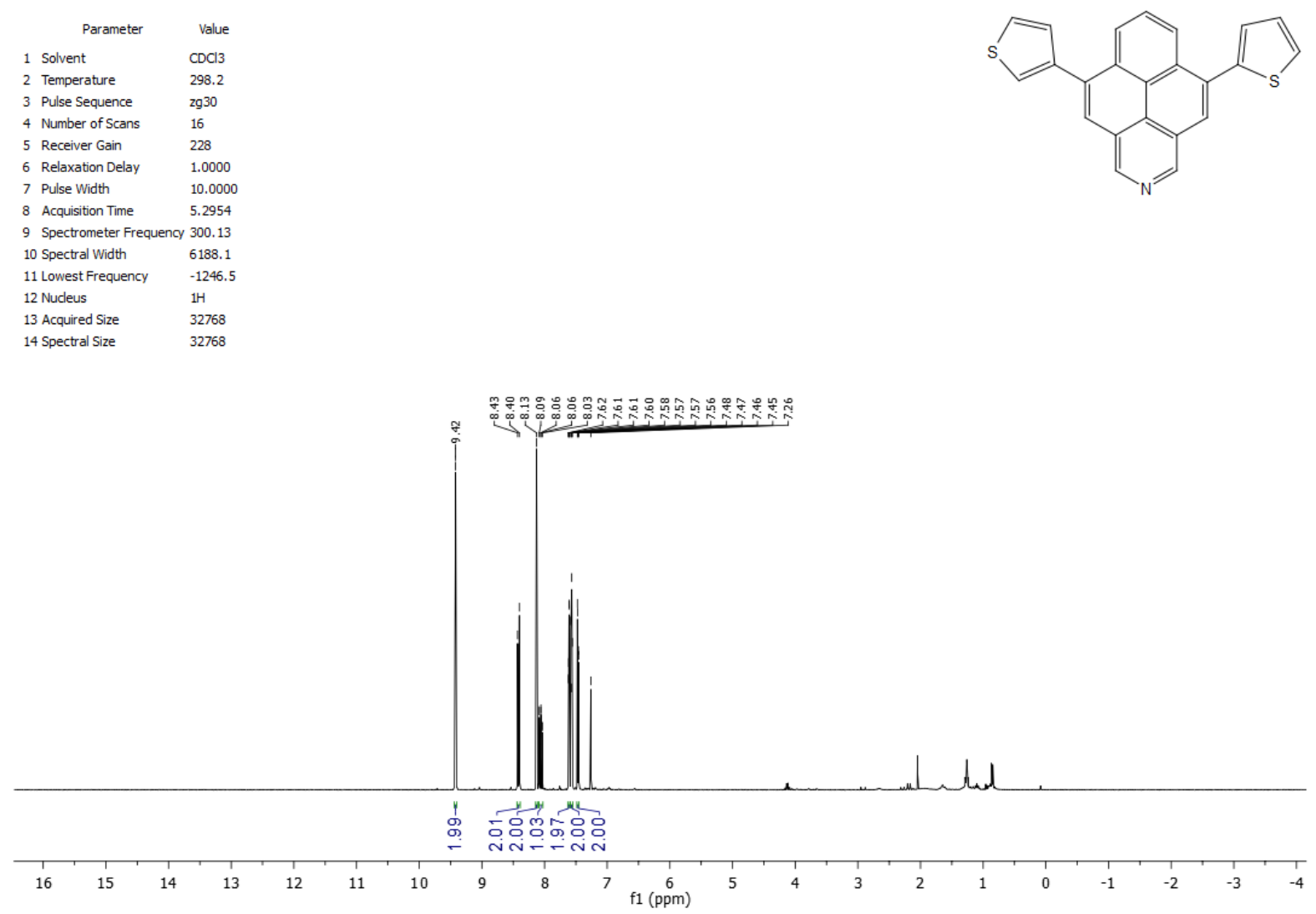

\begin{tabular}{lll}
\multicolumn{1}{c}{ Parameter } & \multicolumn{1}{c}{ Value } \\
1 Solvent & CDCl3 \\
2 Temperature & 298.8 \\
3 Pulse Sequence & 2gpg 30 \\
4 Number of Scans & 1024 \\
5 Seceiver Gain & 2050 \\
6 Relaxation Delay & 2.0000 \\
7 Pulse Width & 10.0000 \\
8 Acquisition Time & 1.8176 \\
9 Spectrometer Frequency & 75.47 \\
10 Spectral Width & 18028.8 \\
11 Lowest Frequency & -1469.9 \\
12 Nucleus & $13 \mathrm{C}$ \\
13 Acquired Size & 32768 \\
14 Spectral Size & 32768
\end{tabular}

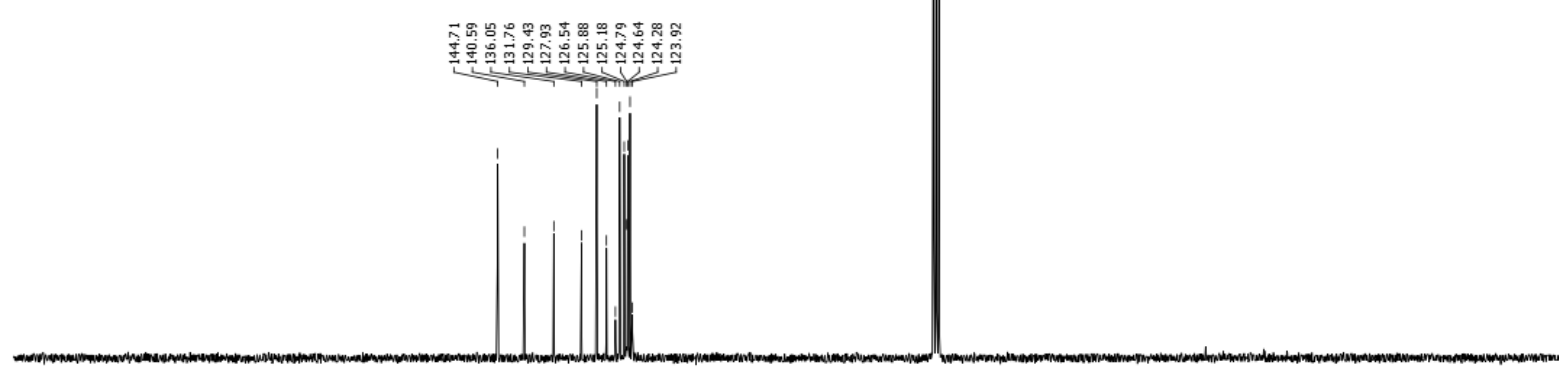

$\begin{array}{llllllllllllllllllllllllll}210 & 200 & 190 & 180 & 170 & 160 & 150 & 140 & 130 & 120 & 110 & 100 & 90 & 80 & 70 & 60 & 50 & 40 & 30 & 20 & 10 & 0 & -10\end{array}$ 